\title{
Theoretical Insights into the Hydrogenation of $\alpha, \beta$-Unsaturated Ketones and Aldehydes to Unsaturated Alcohols over Supported Metal Catalysts
}

A Dissertation
Presented to
The faculty of the School of Engineering and Applied Science
University of Virginia
in partial fulfillment
of the requirement for the Degree
Doctor of Philosophy
in Chemical Engineering
by

\section{Bing Hao}

May

2014 
APPROVAL SHEET

The dissertation

is submitted in partial fulfillment of the requirements

for the degree of

Doctor of Philosophy

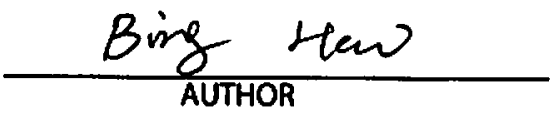

The dissertation has been read and approved by the examining committee:

\begin{tabular}{c} 
Matthew Neurock \\
\hline Advisor \\
Robert Davis \\
\hline John T. Yates \\
\hline Roseanne Ford \\
\hline Gary Koenig
\end{tabular}

Accepted for the School of Engineering and Applied Science:

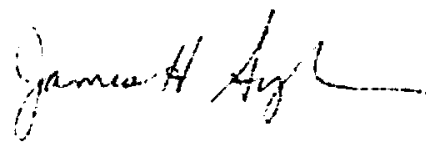

Dean, School of Engineering and Applied Science 


\section{Acknowledgements}

My deepest gratitude goes first to my advisor, Professor Matthew Neurock, for his constant encouragement and commitment to many helpful discussions and excellent insights to this work. I would also like to express my sincere gratitude to all of my lab-mates in Neurock’s group, especially Dr. Nishant Sinha, Dr. Lijun Xu and Dr. Wenjie Tang for their great help during my research work and the writing of this dissertation. Last, but certainly not least, my greatest thanks would go to my beloved parents and my husband, Mengqiang, for their unconditional support, patience, encouragement and love.

Financial support from EPSRC (UK) through CASTech, Johnson \& Matthey, National Science Foundation through CBiRC, and computational support from Pacific Northwest National Laboratory (PNNL), has made this work possible. I would also gratefully acknowledge the collaboration with Dr. Robbie Burch, Dr. Helen Daly, Dr. David Rooney, Dr. Haresh Manyar, Dr. Chris Hardacre at Queens University, Belfast, Dr. Hugh Stitt at Johnson \& Matthey, and Prof. Robert Davis at University of Virginia. 


\begin{abstract}
The selective hydrogenation of $\alpha, \beta$-unsaturated ketones and aldehydes to unsaturated alcohols is an important step in the synthesis of a number of fine chemical and pharmaceutical intermediates as well as in biomass conversion strategies. The ability to selectively hydrogenate $\mathrm{C}=\mathrm{O}$ over $\mathrm{C}=\mathrm{C}$ bonds presents a significant challenge as the hydrogenation of the $\mathrm{C}=\mathrm{C}$ bond is thermodynamically favored over that of the $\mathrm{C}=\mathrm{O}$ bond. Several factors including the structure of reactants, the composition and structure of the metal, the reaction environment (gas/liquid phase reactions) and the support, however, can be tuned in order to influence the elementary kinetic processes and control the catalytic activity and selectivity in the hydrogenation of unsaturated aldehydes and ketones.
\end{abstract}

Periodic density functional theoretical (DFT) studies along with ab initio molecular dynamics and microkinetic simulations were used in this work to explore the selective hydrogenation of $\alpha, \beta$-unsaturated ketones and aldehydes over model catalytic systems. A wide range of saturated and unsaturated aldehydes and ketones and transition metal surfaces were examined in order to understand and analyze the influence of molecular structure of reactant, metal catalyst, the support and the solvent used in liquid phase hydrogenation reactions on the hydrogenation of the $\mathrm{C}=\mathrm{C}$ and $\mathrm{C}=\mathrm{O}$ bonds.

Single substituents such as methyl groups or aromatic rings attached to the $\mathrm{C}=\mathrm{C}$ bond of an aliphatic unsaturated ketone weaken the adsorption of the reactant to the metal surface which lowers the rate of $\mathrm{C}=\mathrm{C}$ hydrogenation, and increases the formation of alkoxide intermediates. The change in a single substituent, however, is not enough to fully reverse the trends in selectivity that favor the formation of the unsaturated alcohol (UA) as there is very little change in the hydrogenation of the alkoxide which is important in the production of unsaturated alcohol. The addition of multiple substitutions at the unsaturated $\mathrm{C}=\mathrm{C}$ bonds on the hydrocarbon backbone such as those found on the highly substituted ring structure of ketoisophorone, can alter the selectivity and enhance the rate of $\mathrm{C}=\mathrm{O}$ hydrogenation over $\mathrm{C}=\mathrm{C}$ hydrogenation. The calculated adsorption energies and the intrinsic activation barriers establish the effects of metal and help to understand the negligible selectivity to the unsaturated alcohol in the hydrogenation of benzalacetone reported over supported $\mathrm{Pt}$ and $\mathrm{Ru}$ catalysts as well as the markedly different selectivities found in the hydrogenation of ketoisophorone over supported Pd and Pt catalysts.

Polar and protic solvents such as water and 10\% mixed isopropyl alcohol-water solvent were found to lower the activation barriers for hydrogenation by stabilizing the partial charges that form in the transition states and alter the preferred reaction mechanism by directly participating in the hydrogenation of unsaturated aldehyde, acrolein, on model Pt catalysts. The presence and operability of a solvent-mediated proton shuttling pathway 
depends on the strength of the hydrogen bonds and is calculated to be dominant for the acrolein hydrogenation in water. Non-polar aromatic solvents such as toluene do not provide hydrogen bonding but can influence the reaction through changing the surface property by blocking the surface sites and thus inhibits the adsorption and ring hydrogenation of benzylacetone reactant. At higher concentrations of toluene, the only available path for hydrogenation is via the $\mathrm{C}=\mathrm{O}$ bond as the adsorbed toluene blocks access to other and such the selectivity to UA increases greatly.

The catalyst support can also act similar to the solvent thus providing additional functionality to enhance $\mathrm{C}=\mathrm{O}$ hydrogenation. Ab initio DFT studies on the hydrogenation of cinnamaldehyde over novel manganese oxide octahedral molecular sieve (OMS-2) suggest that OMS-2 is highly selective for $\mathrm{C}=\mathrm{C}$ bond hydrogenation to form the saturated aldehyde, whereas $\mathrm{Pt}$ supported on OMS-2 favors $\mathrm{C}=\mathrm{O}$ hydrogenation resulting in high selectivity to the unsaturated alcohol. The unique selectivity for Pt/OMS-2 can be attributed to the electron donation effects from $\mathrm{Pt}$ to the OMS-2 support, leading to an activation of $\mathrm{C}=\mathrm{O}$ bond to accept the attack from the hydrogen. The selectivity in hydrogenation of unsaturated oxygenates can thus be tailored by the choice of the metal and its interaction with the OMS-2 support.

This work provides fundamental insights into the atomic scale features of the reagents, the metal surface, the support and the solvent that influence the adsorption of different unsaturated aldehydes and ketones and the subsequent hydrogenation of their $\mathrm{C}=\mathrm{O}$ and $\mathrm{C}=\mathrm{C}$ bonds. 


\section{Table of Contents}

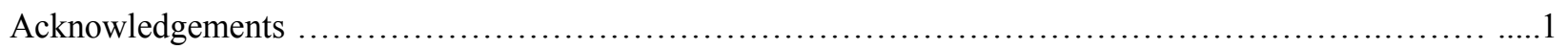

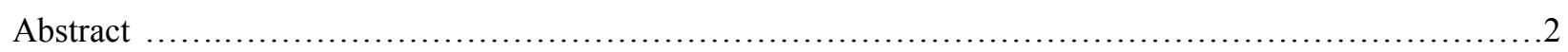

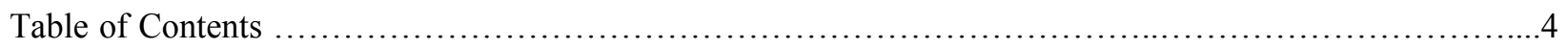

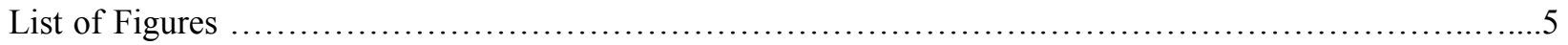

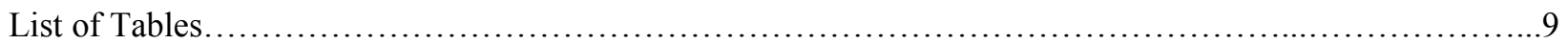

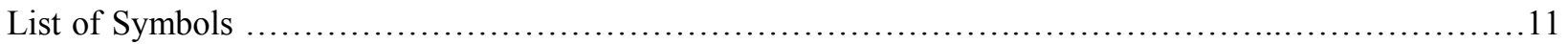

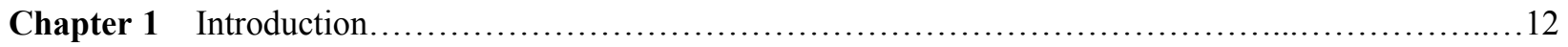

Chapter 2 Influence of the Hydrocarbon Structure on the Hydrogenation of $\alpha, \beta$-Unsaturated Ketones: Substituent and Chain Length Effects .................................................. 17

Chapter 3 Co-operative Effects of the Metal Type and Reactant Structure on the Catalytic Hydrogenations

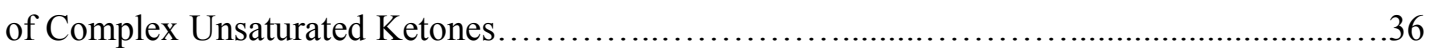

3.1 Hydrogenation of Benzalacetone over Supported Pt and Ru Catalysts............................38

3.2 Hydrogenation of Cyclic Unsaturated Ketones: Ketoisophorone ............................46

Chapter 4 Solvent Effects in Liquid Phase Hydrogenation Reactions.................................55

4.1 Effects of Polar and Protic Solvents: Hydrogenation of Acrolein in Aqueous and Mixed

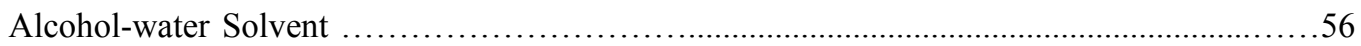

4.2 Effects of Non-Polar Aromatic Solvent: Hydrogenation of Benzylacetone in Toluene ...........71

Chapter 5 Manganese Oxide Octahedral Molecular Sieves (OMS-2) and Pt/OMS-2 as Novel Catalysts for the Hydrogenation of Unsaturated Aldehydes................................................ 79

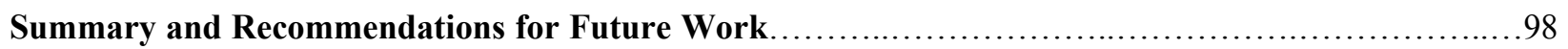

References 102 


\section{Table of Figures}

Figure 2.1 Competitive hydrogenation paths for adsorbed $\alpha, \beta$-unsaturated ketones (UK)

Figure 2.2 Model unsaturated ketones with increasing degree of substitutions at $\mathrm{C}=\mathrm{C}$ bond: (a) methyl vinyl ketone;

(b) isopropenylmethylketone; (c) 3-methyl-3-penten-2-one; (d) 3,4-dimethyl-3-penten-2-one.

Figure 2.3 Adsorption configurations for reaction intermediates of MVK hydrogenation on $\mathrm{Ru}(0001)$.

Figure 2.4 Transition states structures of MVK hydrogenation on $\mathrm{Ru}(0001)$

Figure 2.5 Energetic profiles for the hydrogenation of methyl vinyl ketone (MVK, substitution degree $=0$ ) on

$\mathrm{Ru}(0001)$ surface....

Figure 2.6 The change in the adsorption energies of (a) different adsorption modes of unsaturated ketones and (b) unsaturated alcohols (UA) and saturated ketones (SK) on $\mathrm{Ru}(0001)$ with increasing the degree of substitution at $\mathrm{C}=\mathrm{C}$. .27

Figure 2.7 DFT-calculated transition state structures for IMK hydrogenation on $\mathrm{Ru}(0001)$........ 30

Figure 2.8 DFT-calculated transition state structures for MPO hydrogenation on $\mathrm{Ru}(0001)$. 30

Figure 2.9 DFT-calculated transition state structures for DPO hydrogenation on $\mathrm{Ru}(0001)$ 30

Figure 2.10 DFT-calculated reaction energetic profiles for the hydrogenations of 3,4-dimethyl-3-penten-2-one (DPO, substitution degree=3) on $\mathrm{Ru}(0001)$ surface.

Figure 2.11 Plots of the surface coverages of different intermediates on $\mathrm{Ru}(0001)$ vs. time, via the kinetics analysis at $350 \mathrm{~K}$ for the hydrogenation of (a) MVK $\left(N_{\text {sub }}=0\right)$; (b) DPO $\left(N_{\text {sub }}=3\right)$

Figure 3.1 Competitive hydrogenation paths of (a) MVK, (b) benzalacetone.

Figure 3.2 Adsorption configurations for benzalacetone (a) over $\mathrm{Pt}(111)$ via the aromatic ring and $\mathrm{C}=\mathrm{C}$ bond; (b) over $\mathrm{Pt}(111)$, only via the $\mathrm{C}=\mathrm{O}$ bond; (c) over $\mathrm{Ru}(0001)$ surface......

Figure 3.3 Potential energy profiles of the benzalacetone hydrogenation on (a) $\operatorname{Pt}(111)$ and (b) $\operatorname{Ru}(0001)$.....

Figure 3.4 Transition states configurations for benzalacetone hydrogenation on $\mathrm{Pt}$ (111)

Figure 3.5 Transition states configurations for benzalacetone hydrogenation over $\mathrm{Ru}(0001)$

Figure 3.6 Adsorption configurations for the products of benzalacetone hydrogenation (a) UA on Pt(111); (b) SK on

Pt(111); (c) UA on $\mathrm{Ru}(0001)$; (d) SK on $\mathrm{Ru}(0001)$.

Figure 3.7 Competitive pathways and possible products in the catalytic hydrogenation of ketoisophorone (KIP) over supported Pt and Pd catalysts..... . .46 
Figure 3.8 DFT-calculated adsorption structures of ketoisophorone (KIP). (a) Adsorption via $\mathrm{C}^{2}=\mathrm{C}^{3}$ bond over $\operatorname{Pt}(111)$ surface; (b) via the oxygen $\mathrm{O}^{5}$ of $\mathrm{C}^{4}=\mathrm{O}^{5}$ group over $\mathrm{Pt}(111)$; (c) via the $\mathrm{C}^{2}=\mathrm{C}^{3}$ bond and the oxygen $\mathrm{O}^{5}$ over $\mathrm{Pd}(111)$ surface. .48

Figure 3.9 Competitive reaction pathways for the hydrogenation of ketoisophorone over (a) $\operatorname{Pt}(111)$; (b) $\operatorname{Pd}(111)$

surface.

Figure 3.10 Transition states configurations for the reactions of KIP hydrogenation on Pt (111). .51

Figure 3.11 Transition states configurations for the reactions of KIP hydrogenation on Pd (111)...... .51

Figure 3.12 Adsorption structures for the products of KIP hydrogenations. (a) SK on Pt(111); (b) UA on Pt(111); (c)

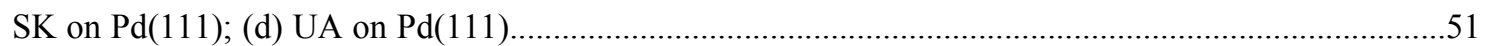

Figure 3.13 Potential energy profiles of the ketoisophorone hydrogenation on (a) $\operatorname{Pt}(111)$ and (b) $\operatorname{Pd}(111)$...... .52

Figure 3.14 Atomic charges at the transition states for the hydrogen attack on (a) the oxygen $\mathrm{O}^{5}$ of the stericallyhindered carbonyl $\mathrm{C}^{4}=\mathrm{O}^{5}$ bond and (b) on the oxygen $\mathrm{O}^{6}$ of the unhindered $\mathrm{C}^{1}=\mathrm{O}^{6}$ bond over $\mathrm{Pt}(111) \ldots 54$

Figure 4.1 (a) Reaction rates for 2-butanone hydrogenation in different pure solvents over $5 \% \mathrm{Pt} / \mathrm{SiO}_{2}$ at $\mathrm{T}=303 \mathrm{~K}$.

(b) Reaction rates and selectivity to unsaturated alcohol product of citral hydrogenation in the mixed IPA-H2O systems over $5 \% \mathrm{Pt} / \mathrm{SiO}_{2}$ at $\mathrm{T}=373 \mathrm{~K}$.

Figure 4.2 Competitive hydrogenation routes of acrolein in vapor phase, in water, and in 10\%IPA-water solvent. $\left(^{\text {a }}\right.$ Metal-mediated hydrogenation path. ${ }^{\mathrm{b}}$ Hydrogenation path via proton shuttling.)

Figure 4.3 The energetic profile of the acrolein hydrogenation on $\operatorname{Pt}(111)$ surface in vapor phase....... .61

Figure 4.4 DFT optimized adsorption configurations of acrolein at the solvent-metal interface over Pt(111) in (a) aqueous phase; (b) 10\% mixed IPA-water solvents (distance in $\AA$ )...

Figure 4.5 Configurations of adsorbed products, unsaturated alcohol in (a) vapor phase; (b) water and (c) mixed IPA/water solvent; and saturated aldehyde in (d) vapor phase; (e) water and (f) mixed solvent. .64

Figure 4.6 Configurations of the intermediates (a) hydroxyl m1; (b) alkoxy m2; (c) alkyl m3 and (d) $\mathrm{m} 4$ of the hydrogenation of acrolein in aqueous phase over $\operatorname{Pt}(111)$.

Figure 4.7 DFT-calculated transition state structures for the hydrogenation of acrolein in aqueous phase over $\operatorname{Pt}(111)$. The hydrogen atom involved in hydrogenation has been shown in yellow (distance in $\AA$ ).

Figure 4.8 DFT-calculated transition state structures for the hydrogenation of acrolein in the mixed IPA/water solvent over $\operatorname{Pt}(111)$

Figure 4.9 Atomic charges and the charge transfer that occurs in the hydrogenation of the oxygen of the $\mathrm{C}=\mathrm{O}$ of acrolein to form hydroxyl intermediate in (a) vapor phase, (b) aqueous phase and (c) 10\% mixed IPAwater solvent. 
Figure 4.10 Charge analysis of the transition states of the proton shuttling mechanism of hydroxyl path in (a) water; (b) mixed IPA/water solvent with 10\% IPA over Pt(111)... . .68

Figure 4.11 Competitive hydrogenation pathways and products of benzylacetone. .72

Figure 4.12 Adsorption energies of toluene, tert-butyl toluene and benzylacetone on $\operatorname{Pt}(111)$ surface in absence of

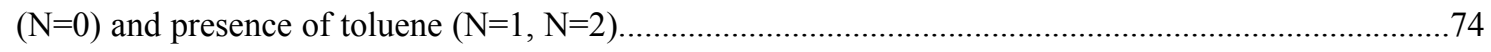

Figure 4.13 Pathways for hydrogenation of benzylacetone on the clean $\operatorname{Pt}(111)$ surface. .75

Figure 4.14 DFT-calculated potential energy profiles of the partial hydrogenation of benzylacetone over clean $\operatorname{Pt}(111)$ surface. .75

Figure 4.15 Activation barriers for the elementary paths in hydrogen addition to the aromatic ring of benzylacetone and toluene on clean $\operatorname{Pt}(111)$ surface. .76

Figure 4.16 Transition states configurations for ring hydrogenation of benzylacetone over clean Pt (111) surface...76

Figure 4.17 Transition states configurations of the $\mathrm{C}=\mathrm{O}$ bond hydrogenation of benzylacetone over clean $\mathrm{Pt}$ (111) surface.

Figure 5.1 (a) Tunnel structures of cryptomelane type OMS-2. (b) Fibrous morphologies of OMS-2 materials. (c) Tunnels of the simulated and observed cryptomelane by HR-TEM.

Figure 5.2 Competitive hydrogenation pathways and products of cinnamaldehyde on 5wt $\%$ Pt/OMS-2 and OMS-2 catalysts.

Figure 5.3 Crystal structures of manganese oxide octahedral molecular sieves (OMS-2) with the $\mathrm{K}^{+}$inside the tunnel.

(a) Top view; (b) perspective view; (c) crystal frameworks.

Figure 5.4 (a) Top view and (b) perspective view of the 3-atomic-layer Pt nanorod supported by the cryptomelane OMS-2 (001) surface model. .84

Figure 5.5 Charge density differences before and after Pt deposition on the OMS-2 surface. Red regions exhibit increasing electronic density, and blue regions exhibit decreasing electronic density . .85

Figure 5.6 Possible hydrogen binding sites over (a) Pt/OMS-2 interface; (b) OMS-2 (001) surface; (c) Pt nanorod..86

Figure 5.7 (a) Spillover of atomic hydrogen from the supported Pt rod to the OMS-2 support along two postulated pathways (b) The corresponding potential energy scheme of diffusion barriers and energy differences...88

Figure 5.8 Four competitive hydrogenation routes of cinnamaldehyde. ' ${ }^{*}$ ' refers to an unsaturated carbon or oxygen that remains bonded to the metal surface after the initial hydrogen addition. . .89

Figure 5.9 Adsorption geometries of cinnamaldehyde on: (a) $\mathrm{Pt}(111)$ surface via the aromatic ring and $\mathrm{C}=\mathrm{C}$ bond; (b) $\mathrm{Pt}(111)$, only via the $\mathrm{C}=\mathrm{O}$ bond; (c) bare OMS-2 (001) surface; (d) on the Pt nanorod supported by 
OMS-2 via the aromatic ring; and (e) on the Pt rod supported by OMS-2, via both the $\mathrm{C}=\mathrm{C}$ and $\mathrm{C}=\mathrm{O}$ bond.

Figure 5.10 Adsorption configurations for the intermediates on the OMS-2 (001) surface and on the Pt nanorod supported by OMS-2.

Figure 5.11 Activation barriers for the initial hydrogenation steps of cinnamaldehyde on the OMS-2 (001) surface, $\mathrm{Pt}(111)$ surface, and the Pt rod supported by OMS-2.

Figure 5.12 Transition state configurations for the hydrogen addition to the $\mathrm{C}=\mathrm{O}$ and $\mathrm{C}=\mathrm{C}$ bonds of cinnamaldehyde on the Pt(111) surface, OMS-2 (001) surface, and the Pt rod supported by OMS-2.......93

Figure 5.13 Electronic effects that result upon the adsorption and reaction of cinnamaldehyde at the Pt/OMS-2 interface.

Figure 5.14 Top and side view of cluster model of Pt/OMS-2 interface, $\mathrm{Pt}_{10}-\mathrm{H}_{32} \mathrm{Mn}_{12} \mathrm{O}_{40} \ldots \ldots \ldots \ldots \ldots \ldots \ldots \ldots \ldots \ldots \ldots . . . . \ldots 5$

Figure 5.15 Atomic charges and the electron transfer that occurs in the hydrogenation of the oxygen of the $\mathrm{C}=\mathrm{O}$ bond, activated by the interfacial $\mathrm{Pt}-\mathrm{Mn}\left(\delta^{+}\right)$bifunctional sites. 


\section{Table of Tables}

Table 2.1 Experimentally reported selectivities at the given conversions for the hydrogenations of $\alpha, \beta$-unsaturated aldehydes and ketones over metal catalysts.

Table 2.2 Influence of the unit cell size (and coverage) on the adsorption energies of MVK onto $\mathrm{Ru}(0001)$. 21

Table 2.3 DFT calculated adsorption energiers and structural propertiesfor the most favorable adsorption modes for the reactants, intermediates and products of the hydrogenations of four unsaturated ketones over $\mathrm{Ru}(0001)$ .25

Table 2.4 DFT calculated activation barriers $\left(E_{a c t}, \mathrm{~kJ} \mathrm{~mol}^{-1}\right)$, reaction energies $\left(E_{r x n}, \mathrm{~kJ} \mathrm{~mol}^{-1}\right)$ and geometric parameters $(\AA)$ of transition states of the elementary steps in hydrogenations of four unsaturated ketones on $\mathrm{Ru}(0001)$. .26

Table 2.5 Simulated steady-state surface coverages of the hydrogenations of MVK $\left(\boldsymbol{N}_{\text {sub }}=0\right)$ and DPO $\left(\boldsymbol{N}_{\text {sub }}=3\right)$ at $350 \mathrm{~K}$. The partial pressure $\mathrm{P}_{\mathrm{MVK}}=25$ Torr and $\mathrm{P}_{\mathrm{H} 2}=500$ Torr.

Table 3.1 Hydrogenation of methyl vinyl ketone (MVK) and benzalacetone over supported Pd, Pt, Ru, and Au catalysts in aqueous $^{\mathrm{a}}$ and ethanol ${ }^{\mathrm{b}}$ solvent.

Table 3.2 Adsorption energies for the reactants (indicated by UK), intermediates and products of benzalacetone hydrogenation on $\mathrm{Pt}(111)$ and $\mathrm{Ru}(0001)$ and MVK hydrogenation on $\mathrm{Ru}(0001)$.

Table 3.3 DFT-calculated adsorption energies for the reactants, intermediates and products of KIP hydrogenation on $\operatorname{Pt}(111)$ and $\operatorname{Pd}(111)$ surfaces. 48

Table 4.1 Characteristic bond lengths $(\AA)$ of the adsorbed acrolein at $\operatorname{Pt}(111)$ in vapor phase and in aqueous and mixed IPA-water solvents.

Table 4.2 DFTcalculated reaction energies and activation barriers $\left(\mathrm{kJ} \mathrm{mol}^{-1}\right)$ of the hydrogenation for acrolein to propenol (UOL) and propanal (SAL) in vapor phase, water and 10\% IPA-water solvent over Pt(111) surface.

Table 4.3 DFT predicted activation barriers $\left(\mathrm{kJ} \mathrm{mol}^{-1}\right)$ and reaction energies $\left(\mathrm{kJ} \mathrm{mol}^{-1}\right)$ of the IPA dehydrogenation in the $10 \%$ mixed IPA-water solvent. .68

Table 4.4 Experimental selectivities (\%) observed in the liquid phase hydrogenations of benzylacetone over supported Pt catalysts in different solvents ${ }^{\mathrm{a}}$ 
Table 4.5 Activation barriers $\left(E_{a c t}, \mathrm{~kJ} \mathrm{~mol}^{-1}\right)$ and reaction energies $\left(E_{r x n}, \mathrm{~kJ} \mathrm{~mol}^{-1}\right)$ for the $\mathrm{C}=\mathrm{O}$ bond hydrogenation of benzylacetone on $\operatorname{Pt}(111)$ surface in absence of $(N=0)$ and presence of toluene $(N=1, N=2)$.

Table 5.1 Experimental selectivities at given conversions for cinnamaldehyde hydrogenation over supported Pt, Ru catalysts and the OMS-2 materials.

Table 5.2 Binding energies (kJ mol-1) of atomic hydrogen at the Pt/OMS-2 interface, bare OMS-2 (001) surface and the stand-alone Pt nanorod

Table 5.3 Adsorption energies for the reactants, intermediates and products of cinnamaldehyde hydrogenation on the bulk Pt(111), bare OMS-2 surface, and the Pt nanorod supported by OMS-2.

Table 5.4 DFT calculated activation barriers and reaction energies for the hydrogenation of cinnamaldehyde on Pt(111) surface, bare OMS-2 (001) surface, and the Pt nanorod supported by OMS-2. . .91 


\section{Table of Symbols}

$\begin{array}{ll}\mathrm{CHG}_{\text {diff }} & \text { Charge density difference }\left(\mathrm{e} / \AA^{3}\right) \\ E_{a c t} & \text { Activation barrier }\left(\mathrm{kJ} \mathrm{mol}^{-1}\right) \\ \Delta E_{a d s} & \text { Adsorption energy }\left(\mathrm{kJ} \mathrm{mol}^{-1}\right) \\ \Delta E_{r x n} & \text { Reaction energy }\left(\mathrm{kJ} \mathrm{mol}{ }^{-1}\right) \\ N & \text { Number of adsorbed solvent molecules per unit cell } \\ N_{s u b} & \text { Degree of Substitution } \\ (a, b, c) & \text { Lattice parameters of crystal structures }(\AA)\end{array}$




\section{Chapter 1 - Introduction}

The selective hydrogenation of carbonyl groups in $\alpha, \beta$-unsaturated aldehydes and ketones is important in the selective formation of unsaturated alcohols, which are valuable intermediates used in the production of fragrances, pharmaceuticals and food flavorings [1-6], as well as in the catalytic reduction of renewable intermediates in the conversion of biomass to value-added chemicals and fuels [7]. The hydrogenation of $\alpha, \beta$ unsaturated ketones, which contain two adjacent double bonds in conjugation, are thought to proceed via a sequence of hydrogen additions to unsaturated bonds to form partially saturated products, including unsaturated alcohols, saturated ketones and fully saturated alcohols. The production and synthesis of unsaturated alcohols requires the selective hydrogenation of the $\mathrm{C}=\mathrm{O}$ bond over the $\mathrm{C}=\mathrm{C}$ bond. A simple gas phase thermodynamics analysis indicates that the hydrogenation of the $\mathrm{C}=\mathrm{C}$ bond to saturated ketone is favored over the hydrogenation of the $\mathrm{C}=\mathrm{O}$ bond [8]. This presents a significant challenge for the selective hydrogenation of the $\mathrm{C}=\mathrm{O}$ group in $\alpha, \beta$-unsaturated aldehydes and ketones.

While the syntheses of unsaturated alcohols from $\alpha, \beta$-unsaturated aldehydes and ketones can be carried in batch processes via classic homogeneous catalysts such as $\mathrm{LiAlH}_{4}$ [9], $\mathrm{Ru}$ complexes [10] or by hydrogentransfer solvents [11], they require the use of environmentally-unfriendly solvents as well as expensive catalyst separation and recovery systems. Therefore, there has been a significant effort to develop heterogeneous catalytic materials that can replace these homogeneous catalytic systems with more efficient and economic processes. The design of heterogeneous catalytic materials that are highly active and selective, however, is rather challenging due to the thermodynamic preference for the hydrogenation of $\mathrm{C}=\mathrm{C}$ over $\mathrm{C}=\mathrm{O}$ bond..

Most of the current efforts in the literature on the selective hydrogenation of unsaturated ketones and aldehydes have focused on the development of supported metal catalysts such as $\mathrm{Pt}, \mathrm{Pd}$, and $\mathrm{Ru}$, due to their ability to actively hydrogenate unsaturated $\mathrm{C}=\mathrm{C}$ and $\mathrm{C}=\mathrm{O}$ bonds [12]. These metals and their alloys, however, have been ineffective in selectively hydrogenating the $\mathrm{C}=\mathrm{O}$ bond over the $\mathrm{C}=\mathrm{C}$ bonds in unsaturated ketones thus resulting in negligible selectivities to the unsaturated alcohols $[12,13]$. There are a few reported cases where higher selectivity can be found for specific starting structures of the reactants [12]. In order to improve the catalytic selectivity along with catalytic activity in the selective hydrogenation of unsaturated ketones and aldehydes, recent efforts have been focused on altering: a) the reactant structure via the addition of substituents onto the $\mathrm{C}=\mathrm{C}$ bond to prevent its adsorption and hydrogenation [13-17]; b) the metal and/or the surface structure of the metal catalyst [18-27]; c) the reaction environment such as by carrying the reactions out in protonic solvent such as water and alcohols [28-32]) and d) the support which can influence the size, and shape of metal particles that form $[12,13]$ and the reactivity at interfacial metal/support sites. How these features control the selective hydrogenation of specific unsaturated ketones is important for understanding the selective 
catalytic hydrogenation of many of the oxygenated molecules of interest in fine chemical synthesis and the conversion of oxygenate molecules derived from renewable intermediates.

a) Structure of Reactants: The catalytic performance of a supported metal catalyst can depended on the structure of the reactant, as the structure can influence the molecules electronic characteristics as well as how it adsorbs onto the surface. Supported monometallic catalysts such as Pd and Pt can selectively hydrogenate some unsaturated aldehydes and ketones that are present in particular ring structures (such as citral) into unsaturated alcohols, but tend to be ineffective in the selective hydrogenation of aliphatic reagents such as acrolein [15] and crotonaldehyde [33]. For reactants with similar structures, the degree of substitution at the $\mathrm{C}=\mathrm{C}$ bond as well as chain length of the substituent can significantly change the overall selectivity on the same catalyst surface. Aliphatic reagents with unsaturated $\mathrm{C}=\mathrm{C}$ and $\mathrm{C}=\mathrm{O}$ substitutents such as acrolein, crontonaldehyde and methyl vinyl ketone all appear to preferentially hydrogenate the $\mathrm{C}=\mathrm{C}$ bonds [13-17] over the $\mathrm{C}=\mathrm{O}$ bonds whereas reactants which contain substituents at their $\mathrm{C}=\mathrm{C}$ bond such as prenal demonstrate higher selectivities towards the hydrogenation of the $\mathrm{C}=\mathrm{O}$ group over $\mathrm{Pt}[14,15]$ and gold catalysts $[16,17]$. This suggests that the presence of substituents onto the $\mathrm{C}=\mathrm{C}$ bond can prevent or impede its adsorption onto the metal surface and thus minimize its hydrogenation, thus increasing the probability of hydrogenating the $\mathrm{C}=\mathrm{O}$ bond to form the desired unsaturated alcohol.

The steric hindrance that results from the presence of bulky substituents attached to the $\mathrm{C}=\mathrm{C}$ bond can influence the selectivity, but it alone cannot explain the poor selectivities to unsaturated alcohols found in hydrogenating most unsaturated ketones with similar bulky substituents over Pd, Pt, and Ru catalysts [13] since these metals can often selectively hydrogenate unsaturated aldehydes with similar sterically hindered $\mathrm{C}=\mathrm{C}$ bonds. Therefore, more studies are needed to establish a more detailed understanding of the structureactivity relationships that control the selective hydrogenation of unsaturated molecules. In this context, theoretical studies on the hydrogenations of model reactants can be used to help establish the influence of substituent and the chain length on the hydrogenation of $\mathrm{C}=\mathrm{C}$ and $\mathrm{C}=\mathrm{O}$ bonds of unsaturated aldehydes and ketones. Preliminary theoretical work on the adsorption [34-37] and hydrogenation [38-44] of acrolein and other unsaturated aldehydes over $\mathrm{Pt}(111)$ [36, 39], $\mathrm{Ag}(110)$ [40] and alloyed $\mathrm{Pt}_{80} \mathrm{Fe}_{20}$ surfaces [38, 41] indicate that the presence of bulky alkyl substituent attached to the $\mathrm{C}=\mathrm{O}$ or $\mathrm{C}=\mathrm{C}$ bonds weakens the interaction of these bonds with the surface [35] and increases their barriers for hydrogenation [44, 45].

b) Influence of the Metal: Selective hydrogenation reactions are typically carried out over supported metal catalysts where structure, composition and the electronic properties of metals can significantly affect the adsorption and the surface reactivity of different reactants and intermediates which can greatly influence the overall activity and selectivity of the catalyst. Among the transition metals that are used in catalytic hydrogenations (e.g. Ru, Rh, Pd, Os, Ir, Pt, $\mathrm{Co}, \mathrm{Ni}, \mathrm{Cu}, \mathrm{Ag}$ and $\mathrm{Au}$ ), Pt, $\mathrm{Ru}$ and $\mathrm{Pd}$ have been the most 
intensively studied metals in the hydrogenation of $\alpha, \beta$-unsaturated aldehydes and ketones [12]. Ru [18-23] and Pt [24-27] demonstrate moderately selectivity toward the formation of unsaturated alcohols from unsaturated aldehydes, while $\mathrm{Pd}$ appears to be the most selective in forming the saturated aldehyde $[18,19,46] . \mathrm{Au} / \mathrm{Fe}_{2} \mathrm{O}_{3}$ has been reported to catalyze the hydrogenation of benzalacetone to the unsaturated alcohol [17]; but the overall rate over gold was extremely low [13] and the exact role of gold is still under debate. Little is known, however, about the structural and electronic features of the metals and how they control catalytic activity and selectivity.

The selective hydrogenation of $\mathrm{C}=\mathrm{O}$ bond over $\mathrm{Pd}, \mathrm{Pt}, \mathrm{Ru}$ and $\mathrm{Rh}$ catalysts can be improved by alloying with promoters of less electronegativity, such as non-transition $\mathrm{Sn}(\mathrm{II}), \mathrm{Ge}(\mathrm{IV}), \mathrm{Ga}(\mathrm{III})$ or transition metal Fe(III) [12, $34,38]$, but the promotional effects for $\alpha, \beta$-unsaturated aldehydes and ketones are not the same. The presence of $\mathrm{Fe}$ atoms in the surface of $\mathrm{Pt}_{80} \mathrm{Fe}_{20}$ alloy facilitates the selective hydrogenations of unsaturated aldehydes to unsaturated alcohol $[38,41]$ but it does not help in the selective hydrogenation of unsaturated ketones [47]. Likewise, the $\mathrm{Sn}$ promoted $\mathrm{Ru} / \mathrm{C}$ and $\mathrm{Pt} / \mathrm{C}$ catalysts were highly selective to unsaturated alcohol in the hydrogenation of unsaturated aldehydes such as cinnamaldehyde and citral, but the hydrogenation of benzalacetone over these catalysts still predominantly leads to the saturated ketone [48-50].

To help establish the factors that control the selectivity, it is important to understand how the reactants as well as the intermediates and products bind to the metal surface and the paths for hydrogen addition. Since the interactions between reactants and metals can considerably differ from one metal to another, the activity and selectivity can also significantly differ from one metal to another and one reactant to another. Although recent theoretical studies have helped to elucidate some of the factors that control the selective hydrogenation of unsaturated aldehydes (e.g., DFT calculations and kinetics analyses have showed that the low selectivity to unsaturated alcohol in acrolein hydrogenation is due to its slow desorption from the surface [51, 52]), there is still little understanding concerning the selective hydrogenation of unsaturated ketones or more generally how changes in the metal or the reactant ultimately dictate the reactivity and selectivity of different $\mathrm{C}=\mathrm{C}$ and $\mathrm{C}=\mathrm{O}$ bonds.

c) Reaction Environment - Solvent Effects: As hydrogenation involves the transfer of hydrogen to the reaction center, protic solvents such as water and alcohol can often enhance the reactivity as a result of hydrogen bonding, solvation effects and the ability the transfer protons. In fact, many important hydrogenation processes are carried out in a solvent over supported metal catalysts. Solvents are known to affect the reaction rate and selectivity [6] through a variety of cooperative effects such as solvent polarity, hydrogen solubility, mass transfer, solvation of reactants in bulk liquid-phase [12] and stabilization of the partially charged transition states by the polar solvent [28]. Recent work on polar and protic solvents revealed that among pure solvents, water is significantly better than alcohols and alkanes in the hydrogenation of carbonyls. Water can 
also alter the preferred reaction pathways via facilitating proton shuttling mechanisms typically found in biological systems thus involving proton-coupled-electron transfer from the metal site to the reaction center via a chain of water molecules [31]. Initial studies of the mixed water/alcohol solvents showed some correlation between reactivity with factors like proton diffusivity, donor/acceptor number[53] and dipole moment[32], but the exact role of protic solvents has not been unraveled. A systematic study on the effects of the solvent on each of the elementary steps involved in the hydrogenation of $\mathrm{C}=\mathrm{O}$ and $\mathrm{C}=\mathrm{C}$ bonds would help to provide a strong fundamental basis of how the reaction media can be used to control the selective hydrogenation of aldehydes and ketones.

Non-polar solvents such as toluene can not provide facile proton shuttling paths or the stability from hydrogen bonding like those of protic solvents but can affect the reactivity and selectivity via altering the surface property of the catalyst such as by blocking the surface sites via the strong binding between the aromatic rings and the metal surfaces [54]. A clear understanding on the effects of non-polar aromatic solvents on the catalytic activity of popular metal catalysts is quite demanding, where the first principle DFT calculations with model catalyst systems can begin to provide important insights.

d) Effects of Supports and the Metal/Support Interface: The properties and composition of the support can also play an important role in influencing the catalytic behavior in selective hydrogenation reactions. The support is not only important in the dispersion of and stabilization of metal particles that carry out catalysis but can also play a role in influencing the electronic structure of the active metal sites and alter the solvent behavior at the metal/support interface and in some instances directly participate in the surface reactions. The importance of support effects on the hydrogenation of unsaturated aldehydes and ketones has been suggested in a number of previous experimental studies [12, 13, 16, 18-23]. The support can influence the selectivity for $\mathrm{C}=\mathrm{C}$ and $\mathrm{C}=\mathrm{O}$ hydrogenation. $\mathrm{Ru} / \mathrm{C}$ and $\mathrm{Ru} / \mathrm{SiO}_{2}$, for example, show $>20 \%$ higher selectivities to the unsaturated alcohol over $\mathrm{Ru} / \mathrm{Al}_{2} \mathrm{O}_{3}$ for cinnamaldehyde hydrogenation [18, 22]. Unfortunately, most conventional supports such as oxides and carbons have not been able to selective promote the hydrogenation of the $\mathrm{C}=\mathrm{O}$ bond over the $\mathrm{C}=\mathrm{C}$ bond for unsaturated ketones and as a result the selectivity to the desired unsaturated alcohols remain very low $[12,13]$.

The design of supports that would selectively interact with the $\mathrm{C}=\mathrm{O}$ group over the $\mathrm{C}=\mathrm{C}$ bond could result in significant enhancement in the selectivity to hydrogenate unsaturated ketones and aldehydes to unsaturated alcohols. In this context, the octahedral molecular sieves of manganese oxides (OMS-2) [55, 56] are highly promising materials. Their multivalent character and $2 \times 2$ tunnel structure with lattice oxygen vacancies making them potential redox catalysts[57, 58] [59]. OMS-2 can also act as support to anchor metal particles such as Pt which can readily split hydrogen thus providing bifunctional sites that may improve the catalytic performance. Preliminary efforts by our collaborators at Queens University suggest that these materials are 
both active and selective [60]. Herein we will use theory in order to understand possible active sites and reaction pathways and mechanisms for these materials to understand how they might promote the selective hydrogenation reactions.

In this work, we have applied periodic density functional theory (DFT) [61, 62] studies along with ab initio molecular dynamics simulation and microkinetic simulation to explore the selective hydrogenation of $\alpha, \beta$ unsaturated ketones and aldehydes over model catalytic systems. A variety of molecules and transition metal surfaces are examined in order to understand and analyze the influence of molecular structure of reactant (Chapter 2), metal type of catalysts (Chapter 3), solvent effects in liquid phase hydrogenation (Chapter 4), and the influence of the support and the metal/support interface (Chapter 5) on the hydrogenation of the $\mathrm{C}=\mathrm{C}$ and $\mathrm{C}=\mathrm{O}$ bonds of unsaturated aldehydes and ketones. This work will provide fundamental understanding of the atomic scale features of the reagents, the metal surface, the support and the solvent that influence the adsorption and hydrogenation of $\mathrm{C}=\mathrm{O}$ and $\mathrm{C}=\mathrm{C}$ bonds. 


\section{Chapter 2 - Influence of the Hydrocarbon Structure on the Hydrogenation of $\alpha, \beta$ - Unsaturated Ketones: Substituent and Chain Length Effects}

Substituents at the $\mathrm{C}=\mathrm{C}$ bond of an unsaturated ketone lower the rate of $\mathrm{C}=\mathrm{C}$ bond hydrogenation and increase the production rate of alkoxide intermediate, but do not significantly increase the hydrogenation of alkoxide, which is important in the production of desired unsaturated alcohol (UA). The $\mathrm{C}=\mathrm{C}$ bond hydrogenation is still more favorable than that of the $\mathrm{C}=\mathrm{O}$ bond on $\mathrm{Ru}$ catalyst, which rationalizes the observed low selectivity to UA in experiments.

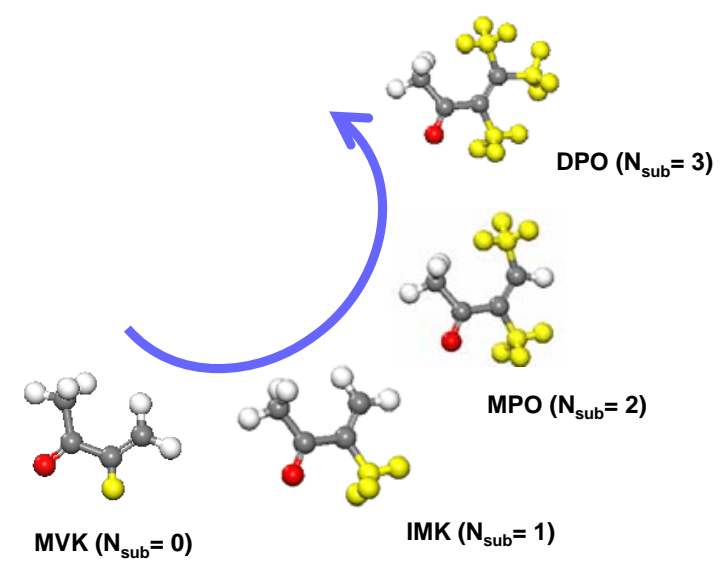

\section{INTRODUCTION}

The selective hydrogenation of the carbonyl $\mathrm{C}=\mathrm{O}$ bond over the $\mathrm{C}=\mathrm{C}$ bond in $\alpha, \beta$-unsaturated ketones to unsaturated alcohol is an important but challenging task, since the hydrogenation of the $\mathrm{C}=\mathrm{C}$ bond is thermodynamically more favorable [63]. The $\alpha, \beta$-unsaturated ketones are comprised of two adjacent double bonds in conjugation and can follow any one of the four reaction paths depicted in Figure 2.1, resulting in the competitive formation of partially saturated products unsaturated alcohols (UA), saturated ketone (SK), and fully saturated alcohol (SA). Recent experimental results which are summarized in Table 2.1 suggest that the selective hydrogenation of the carbonyl groups in most unsaturated ketones and aldehydes, including acrolein, crontonaldehyde, methyl vinyl ketone and 3-penten-2-one, does not occur over conventional monometallic catalysts such as Pt and Ru [13-17]. There are, however, a few very notable exceptions which include prenal and 4-methyl-3-penten-2-one which undergo high selectivity to unsaturated alcohol over Pt $[14,15]$ and gold catalysts $[16,17]$. These molecules all have substituents at the unsaturated $\mathrm{C}=\mathrm{C}$ bond which can inhibit their adsorption and reaction on the metal surface and increase the probability of hydrogenating the $\mathrm{C}=\mathrm{O}$ bond to form the desired unsaturated alcohol. Steric hindrance alone, however, cannot explain why bulky substituents do not increase the selectivity to unsaturated alcohol for the hydrogenation of other unsaturated ketones over $\mathrm{Pd}, \mathrm{Pt}$, and Ru catalysts [13] since these metals can sometimes selectively hydrogenate unsaturated aldehydes with similar sterically hindered $\mathrm{C}=\mathrm{C}$ bonds. While the reported experimental results provide invaluable 
evidence that substituents can affect hydrogenation selectivity, there is little insight into how these subsitutents influence the elementary adsorption and surface reaction steps that control the overall catalytic behavior.

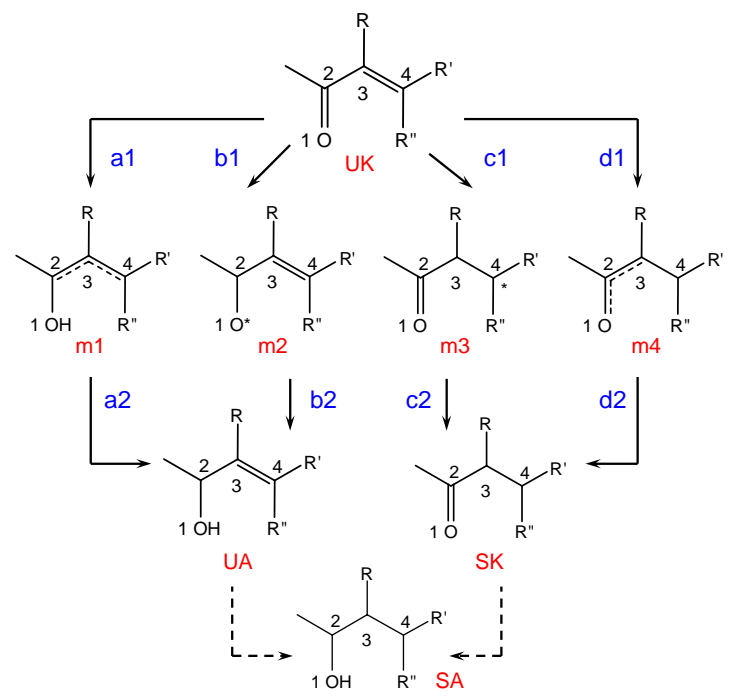

Figure 2.1 Competitive hydrogenation paths for adsorbed $\alpha, \beta$-unsaturated ketones (UK). The four possible sites for the the addition of hydrogen to the unsaturated $\mathrm{C}$ and $\mathrm{O}$ atoms are numbered from 1 to 4 . The first hydrogen addition steps (a1, b1, c1, d1) provide four mono-hydrogenated intermediates (m1- m4, named after the atom number to which the $\mathrm{H}$ adds), and the subsequent hydrogen addition steps (a2, b2, c2, d2) that result in the formation of the unsaturated alcohols (UA) and saturated ketones (SK) products. Both UA and SK can further react to form the saturated alcohols (SA).

Table 2.1 Experimentally reported selectivities at the given conversions for the hydrogenations of $\alpha, \beta$-unsaturated aldehydes and ketones over metal catalysts. UA: unsaturated alcohol; SK: saturated ketone; SAL: saturated aldehyde.

\begin{tabular}{|c|c|c|c|c|c|c|}
\hline Reactant & Structure & Catalyst & UA (\%) & SK/SAL (\%) & Conversion $(\%)$ & Ref. \\
\hline Acrolein & & $\begin{array}{l}\mathrm{Pt} / \mathrm{Aerosil} \\
\mathrm{Pt} / \mathrm{Al}_{2} \mathrm{O}_{3}\end{array}$ & $\begin{array}{c}<2 \\
0\end{array}$ & $\begin{array}{l}93 \\
95\end{array}$ & $\begin{array}{l}<10 \\
<5\end{array}$ & $\begin{array}{l}{[14]} \\
{[15]}\end{array}$ \\
\hline Crotonaldehyde & & $\begin{array}{c}\mathrm{Pt} / \text { Aerosil } \\
\mathrm{Pt} / \mathrm{C} \\
\mathrm{Ru} / \mathrm{C}\end{array}$ & $\begin{array}{l}13 \\
10 \\
17\end{array}$ & $\begin{array}{l}50 \\
85 \\
76\end{array}$ & $\begin{array}{c}<20 \\
68 \\
61\end{array}$ & $\begin{array}{l}{[14]} \\
{[13]} \\
{[13]}\end{array}$ \\
\hline Prenal & & Pt/Aerosil & 21 & 17 & $<20$ & [15] \\
\hline Methyl vinyl ketone & & $\begin{array}{l}\mathrm{Pt} / \mathrm{C} \\
\mathrm{Ru} / \mathrm{C}\end{array}$ & $\begin{array}{l}0 \\
0\end{array}$ & $\begin{array}{l}98 \\
98\end{array}$ & $\begin{array}{l}89 \\
72\end{array}$ & [13] \\
\hline 3-penten-2-one & & $\mathrm{Au} / \mathrm{Fe}_{2} \mathrm{O}_{3}$ & 16 & 60 & 90 & {$[16,17]$} \\
\hline $\begin{array}{l}\text { 4-methyl-3-penten-2- } \\
\text { one }\end{array}$ & & $\mathrm{Au} / \mathrm{Fe}_{2} \mathrm{O}_{3}$ & 61 & 35 & 22 & {$[16,17]$} \\
\hline
\end{tabular}


Theoretical studies have been quite useful in establishing the change in catalytic selectivity for the hydrogenation of unsaturated aldehydes with substitution groups. Recent DFT calculations of the adsorption [34-37] and hydrogenation [38-44] of acrolein and other unsaturated aldehydes over model metal catalysts of $\operatorname{Pt}(111)[36,39], \operatorname{Ag}(110)$ [40] and the alloyed $\mathrm{Pt}_{80} \mathrm{Fe}_{20}$ surfaces [38, 41] suggest that the presence of bulky alkyl substituents attached to the alkene groups protect the $\mathrm{C}=\mathrm{C}$ double bonds by weakening the overall adsorption of the molecule [35] and increasing the barriers for $C=C$ hydrogenation $[44,45]$. The calculated activation barriers for the initial hydrogen addition were shown to directly follow the strength of adsorption of the initial reactant [45]. These previous studies, however, have all been focused on the reactions of unsaturated aldehydes with little to no insight into the hydrogenation of the unsaturated ketones. In this work, we carry out systematic density functional theoretical $[61,62]$ calculations in order to explore the reactivity trends for a series of model ketones over model transition metal surfaces $\mathrm{Ru}(0001)$. $\mathrm{Ru}$ was used as it is suggested to be one of the most active metal catalyst for the selective hydrogenation of carbonyl species, especially in the presence of water [64].

We start with the simplest unsaturated ketone, methyl vinyl ketone (MVK) and use first principle density functional theory calculations to examine the effects of methyl substituents on the unsaturated $\mathrm{C}=\mathrm{C}$ bond on the adsorption energies and reaction kinetics for a series of model ketones over the $\operatorname{Ru}(0001)$ surface. The model unsaturated ketones that were examined are shown in Figure 2.2. They include MVK, isopropenyl methyl ketone (IMK), 3-methyl-3-penten-2-one (MPO) and 3,4-dimethyl-3-penten-2-one (DPO). For the sake of simplicity, all of the DFT calculated reactions reported herein were carried out in vapor phase and on an idealized clean single crystal surface at low coverage. Factors such as high surface coverage of reactants [65] and the presence of solvents $[28,32,66,67]$ that related to the difference between idealized environments examined herein and the actual catalytic systems can contribute to the differences in reported reaction energetics.

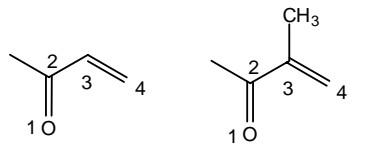

(a) (b)

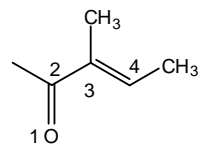

(c)

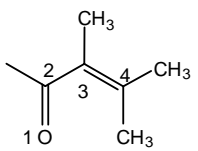

(d)

Figure 2.2 Model unsaturated ketones with increasing degree of substitutions at $\mathrm{C}=\mathrm{C}$ bond: (a) methyl vinyl ketone (MVK); (b) isopropenyl methyl ketone (IMK); (c) 3-methyl-3-penten-2-one (MPO); (d) 3,4-dimethyl-3-penten-2one (DPO). Carbon atoms are numbered starting from the one nearest to the oxygen. 


\section{METHODOLOGY}

The adsorption energies, reaction energies and activation barriers associated with the reaction intermediates and pathways reported in Figure 2.1 were all calculated by carrying out first principle density functional theory. All the reported herein were carried out using the Vienna ab initio simulation package (VASP) [68]. The Perdew-Wang 91 form of generalized gradient approximation [69] was used to calculate non-local gradient corrections to the correlation and exchange energies. The wave function was constructed from an expansion of plane waves with an energy cutoff of $396 \mathrm{eV}$. The electron-ion interactions in core region were described by Vanderbilt ultrasoft pseudopotentials with real space projection operators [70]. The energies of absorbates in vacuum were calculated spin-polarized in a $16 \times 16 \times 16 \AA$ unit cell. All of the calculations on the nonmagnetic ruthenium surfaces, $\mathrm{Ru}(0001)$, were carried out non-spin-polarized. The geometry optimizations were carried out via a three-step procedure, where the first step converged the atomic structures to a point where the forces on all atoms were less than $0.10 \mathrm{eV} / \AA$, followed by a second optimization step to reduce the forces to 0.05 $\mathrm{eV} / \AA$ with the wave functions converged to $1 \times 10^{-6} \mathrm{eV}$. Both the first and second steps were performed using $3 \times 3 \times 1$ Monkhorst-Pack k-point mesh [71] to sample the Brillouin zone integration [72], whereas the third and final step was carried out using a $6 \times 6 \times 1 \mathrm{k}$-point mesh as static single-point calculations, to determine the lowest energy structure and ensure a higher level of accuracy on the electronic energy.

The bulk lattice constant for Ru was initially set to the experimental value of $a=2.71 \AA$ [73] and then optimized within VASP. The optimized values of $a=2.70 \AA, \mathrm{c}=4.28 \AA$ were subsequently used in the construction of the $\mathrm{Ru}(0001)$ surfaces. The surface was modeled using a $3 \times 3$ unit cell to accommodate the reacting species and minimize spurious cell-to-cell interactions, with four metal layers and a $16 \AA$ vacuum region to separate the slabs in the z-direction. The top two metal layers were allowed to relax, while the bottom two layers were fixed to their optimized bulk lattice positions. Adsorption was examined only at the top surface of the slab. The adsorption energies were calculated as $\Delta E_{\text {ads }}=E_{\text {metal }+ \text { adsorbate }}-E_{\text {adsorbate }}-E_{\text {metal }}$, where a negative value indicates that the adsorption is exothermic. The calculations were carried out at a constant surface coverage of 1/9 species ML ( 1 adsorbate per $3 \times 3$ unit cell), which is much lower than the saturation coverage to avoid strong repulsive interaction between adsorbates. Adsorption energies of adsorbate in different sizes of unit cells were compared in Table 2.2, probed by the MVK molecule. The relative error in using $3 \times 3$ unit cell rather than $4 \times 4$ unit cell was found to be $\sim 1 \%$.

The transition state, the saddle point on the minimum energy path (MEP), was located through combining the nudged elastic band (NEB) method [74-77] to establish images along the MEP that bracket the transition state and the Dimer method [78-80] to isolate the specific transition state. In NEB calculation, an initial series of intermediate images were refined using a $3 \times 3 \times 1$ k-point mesh until the maximum force on each atom 
decreased to $0.20 \mathrm{eV} / \AA$. The dimer was formed by system replicas with very small distance between them (set to $0.01 \AA$ ), and rotated through a minimization of the torque to ensure that the dimer is in-line with the MEP. The dimer calculations were carried out by $3 \times 3 \times 1 \mathrm{k}$-point until the maximum atomic forces converged to $0.05 \mathrm{eV} / \AA$, and finally a $6 \times 6 \times 1 \mathrm{k}$-point was used to determine the energy of the transition state (TS) in a static single-point calculation. All of the transition states involved in the hydrogenation of MVK were confirmed by carrying out vibrational frequency calculations to show that there was only one imaginary frequency corresponding to the reaction path. The transition states for IMK, MPO and DPO were found to be very similar to MVK and as such we did not carry out vibrational frequency analyses on the DPO intermediates. The intrinsic activation barriers and overall reaction energies were determined by calculating the energy difference between the transition state (TS) and the reference state (adsorbate and $\mathrm{H}$ atom at an infinite distance on the surface) and the product and the reference state, respectfully.

Table 2.2 Influence of the unit cell size (and coverage) on the adsorption energies of MVK onto Ru(0001).

\begin{tabular}{cccc}
\hline Size of unit cell & Coverage & $\Delta E_{a d s}\left(\mathrm{~kJ} \mathrm{~mol}^{-1}\right)$ & Relative error \\
\hline $2 \times 2$ & $1 / 4$ & -140 & $6.4 \%$ \\
$3 \times 3$ & $1 / 9$ & -148 & $1.2 \%$ \\
$4 \times 4$ & $1 / 16$ & -150 & - \\
\hline
\end{tabular}

\section{RESULTS AND DISCUSSION}

The elementary steps involved in the sequential hydrogenation of the $\mathrm{C}=\mathrm{C}$ and $\mathrm{C}=\mathrm{O}$ bonds in four model unsaturated ketones over $\mathrm{Ru}(0001)$ surface were investigated by carrying out DFT calculations. We first studied the hydrogenation of the simplest unsaturated ketone, MVK (substitution degree at $\mathrm{C}=\mathrm{C}$ bond $\boldsymbol{N}_{\text {sub }}=0$ ).

\section{1) Hydrogenation of Methyl vinyl ketone}

The hydrogenation of MVK was assumed to proceed from its most stable adsorption state on the $\mathrm{Ru}(0001)$ surface, where MVK is oriented parallel to the surface in a planar $\eta^{4}$-cis configuration (Figure2.6 a) which allows both the $\mathrm{C}=\mathrm{C}$ and $\mathrm{C}=\mathrm{O}$ bonds to interact directly with four $\mathrm{Ru}$ atoms in the surface. A similar $\eta^{4}$ adsorption mode was found in the adsorption of different unsaturated aldehydes (acrolein, crotonaldehyde and prenal) on $\mathrm{Pt}(111)$ [35]. The first hydrogen atom can add to either the oxygen $\left(\mathrm{O}^{1}\right)$ or carbon $\left(\mathrm{C}^{2}\right)$ atoms of the $\mathrm{C}=\mathrm{O}$ bond, or to either the secondary $\left(\mathrm{C}^{3}\right)$ or primary $\left(\mathrm{C}^{4}\right)$ carbon atoms of the $\mathrm{C}=\mathrm{C}$ bond, to form the $\mathrm{m} 1, \mathrm{~m} 2$, $\mathrm{m} 3$ and $\mathrm{m} 4$ intermediates shown in Figure 2.1 via paths a1, b1, c1, and d1 respectively. The addition of the second hydrogen atom to either the partially saturated $\mathrm{C}=\mathrm{O}$ or the $\mathrm{C}=\mathrm{C}$ bond produces the unsaturated alcohol 
(UA) or saturated ketone (SK). The saturated alcohol (SA) is produced by the sequential hydrogenation of either of the intermediate UA or SK products. All four of the partially hydrogenated alkoxy or alkyl intermediates bind to the $\mathrm{Ru}$ surface via the remaining unsaturated $\mathrm{C}$ or $\mathrm{O}$ atom in the activated $\mathrm{C}=\mathrm{C}$ or $\mathrm{C}=\mathrm{O}$ bond along with the unaltered $\mathrm{C}=\mathrm{C}$ or $\mathrm{C}=\mathrm{O}$ bonds thus resulting in the formation of $\eta^{3}$ adsorption structures shown in Figure 2.3 a-d. The adsorption energies and characteristic bond lengths for all four of these intermediates as well as the reactants and products (UA and SK) are reported in Table 2.3.
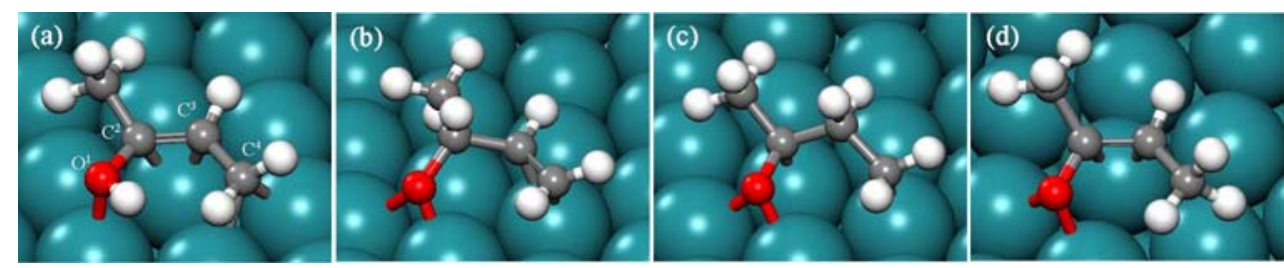

Figure 2.3 Adsorption configurations for reaction intermediates (a) $\mathrm{m} 1$; (b) $\mathrm{m} 2$; (c) $\mathrm{m} 3$; (d) $\mathrm{m} 4$ of MVK hydrogenation on $\mathrm{Ru}(0001)$.
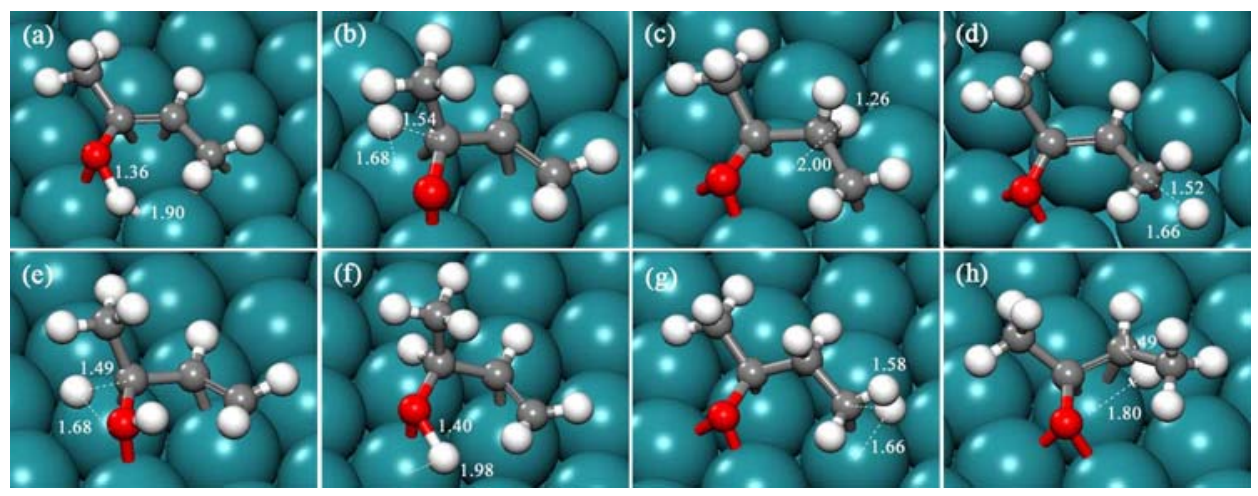

Figure 2.4 Transition state structures for the hydrogen addition to: (a) $\mathrm{O}^{1}$ (path a1); (b) $\mathrm{C}^{2}$ (path b1); (c) $\mathrm{C}^{3}$ (path c1); (d) $\mathrm{C}^{4}$ (path d1); (e) $\mathrm{C}^{2}$ (path a2); (f) $\mathrm{O}^{1}$ (path b2); (g) $\mathrm{C}^{4}$ (path c2); (h) $\mathrm{C}^{3}$ (path d2) for MVK hydrogenation on $\mathrm{Ru}(0001)$.

The DFT-calculated activation barriers for the addition of the first hydrogen to the $\mathrm{C}=\mathrm{C}$ and $\mathrm{C}=\mathrm{O}$ of adsorbed MVK reported in Table 2.4 increase in the following order:

$\mathrm{C}^{4}$ path d1 $\left(77 \mathrm{~kJ} \mathrm{~mol}^{-1}\right)<\mathrm{C}^{2}-(\mathrm{O})$ path b1 $\left(99 \mathrm{~kJ} \mathrm{~mol}^{-1}\right)<\mathrm{C}^{3}$ path c1 $\left(109 \mathrm{~kJ} \mathrm{~mol}^{-1}\right)<\mathrm{O}^{1}$ path a1 $\left(115 \mathrm{~kJ} \mathrm{~mol}^{-1}\right)$

This ordering is largely dictated by the atom type (carbon or oxygen) and degree of substitution at the atom to which the hydrogen is added, and the stabilization of the transition states as a result of the creation of the stronger $\mathrm{Ru}-\mathrm{O}$ bonds during alkoxide formation $(\mathrm{m} 2)$ as compared to the weaker Ru-CHR bonds during alkyl (m3 and $\mathrm{m} 4)$ or hydroxyalkyl formation (m1). 
The activation barrier to add the first hydrogen to the primary (terminal) carbon $\mathrm{C}^{4}$ of the $\mathrm{C}=\mathrm{C}$ bond was calculated to be the lowest one at $77 \mathrm{~kJ} \mathrm{~mol}^{-1}$ (path $\mathrm{d} 1$ ), since the addition of hydrogen to an $\mathrm{sp}^{2}$ carbon atom is much more favored than the addition to an $\mathrm{sp}^{2}$ oxygen atom and the primary carbon has the fewest substituents and thus the lowest steric repulsion (Figure $2.4 \mathrm{~d}$ ).

The barriers to hydrogenate the secondary carbon center of the $\mathrm{C}=\mathrm{C}$ (path $\mathrm{c} 1$ ) and carbon center of $\mathrm{C}=\mathrm{O}$ bond (path b1) to form the $\mathrm{m} 3$ alkyl and $\mathrm{m} 2$ alkoxide intermediates are considerably higher than the barrier to add to the primary carbon (path $\mathrm{d} 1$ ) to form the $\mathrm{m} 4$ alkyl intermediate. This trend is the result of the higher degree of steric hindrance as well as the higher bond orders at the more substituted $\mathrm{C}$ centers, which decrease their stabilization by the surface in the transition state.

The barrier for the addition of $\mathrm{H}$ to the tertiary carbon of the carbonyl $\left(99 \mathrm{~kJ} \mathrm{~mol}^{-1}\right)$ is actually $10 \mathrm{~kJ} \mathrm{~mol}^{-1}$ lower than that for the addition to the secondary carbon of the $\mathrm{C}=\mathrm{C}$ bond $\left(109 \mathrm{~kJ} \mathrm{~mol}^{-1}\right)$. The higher degree of steric hindrance at the tertiary carbon $\mathrm{C}^{2}$ over the secondary carbon $\mathrm{C}^{3}$ is overcome by the greater stabilization of the transition state, as a result of the creation of the strong Ru-O bond during the formation of the alkoxide intermediate over that of Ru-CHR bond creation during the formation of the alkyl intermediate (Figure $2.4 \mathrm{~b}-\mathrm{c}$ ). The difference is also reflected in the much stronger binding of the alkoxide intermediate $\left(-300 \mathrm{~kJ} \mathrm{~mol}^{-1}\right.$ for m2) over that of the alkyl intermediate $\left(-195 \mathrm{~kJ} \mathrm{~mol}^{-1}\right.$ for $\left.\mathrm{m} 3\right)$ that forms.

The highest initial activation barrier occurs for the addition of hydrogen to the $\mathrm{O}$ atom of the carbonyl (path a1), which has a barrier of $115 \mathrm{~kJ} \mathrm{~mol}^{-1}$. This is the result of the intrinsic differences in forming the O-H bond versus the $\mathrm{C}-\mathrm{H}$ bond on $\mathrm{Ru}$, and in addition, to the weaker stabilization of the transition state (Figure 2.4a) by the surface to form the hydroxyalkyl compared to that of the alkoxide or the alkyl intermediates.

The addition of the second hydrogen atom to either the partially saturated $\mathrm{C}=\mathrm{O}$ (steps a2 or b2 in Figure 2.1) or $\mathrm{C}=\mathrm{C}$ (steps $\mathrm{c} 2$ or $\mathrm{d} 2$ ) bonds produces the unsaturated alcohol (UA) or saturated ketone (SK). The barriers for each of these steps on $\mathrm{Ru}(0001)$ are shown in Table 2.4. The addition of hydrogen to the primary alkyl intermediate via path $\mathrm{c} 2$ has the lowest barrier $\left(63 \mathrm{~kJ} \mathrm{~mol}^{-1}\right)$ among all steps examined because of the favorable access to the primary carbon center that has very limited steric hindrance to $\mathrm{H}$ addition as well as to the fact that it has the lowest bond order. The addition of hydrogen to the alkoxide intermediate (m2) in path b2 was calculated to be the most difficult as $114 \mathrm{~kJ} \mathrm{~mol}^{-1}$. This step requires the breaking of a very strong Ru-O bond, which is over $100 \mathrm{~kJ} \mathrm{~mol}^{-1}$ stronger than any Ru-O bond in the other three alkyl intermediates. The barriers to hydrogenate the secondary and tertiary alkyl intermediates shown in paths a2 $\left(86 \mathrm{~kJ} \mathrm{~mol}^{-1}\right)$ and $\mathrm{d} 2\left(87 \mathrm{~kJ} \mathrm{~mol}^{-1}\right)$ both have modest barriers that fall between those for paths b2 $\left(114 \mathrm{~kJ} \mathrm{~mol}^{-1}\right)$ and $\mathrm{c} 2\left(63 \mathrm{~kJ} \mathrm{~mol}^{-1}\right)$. 


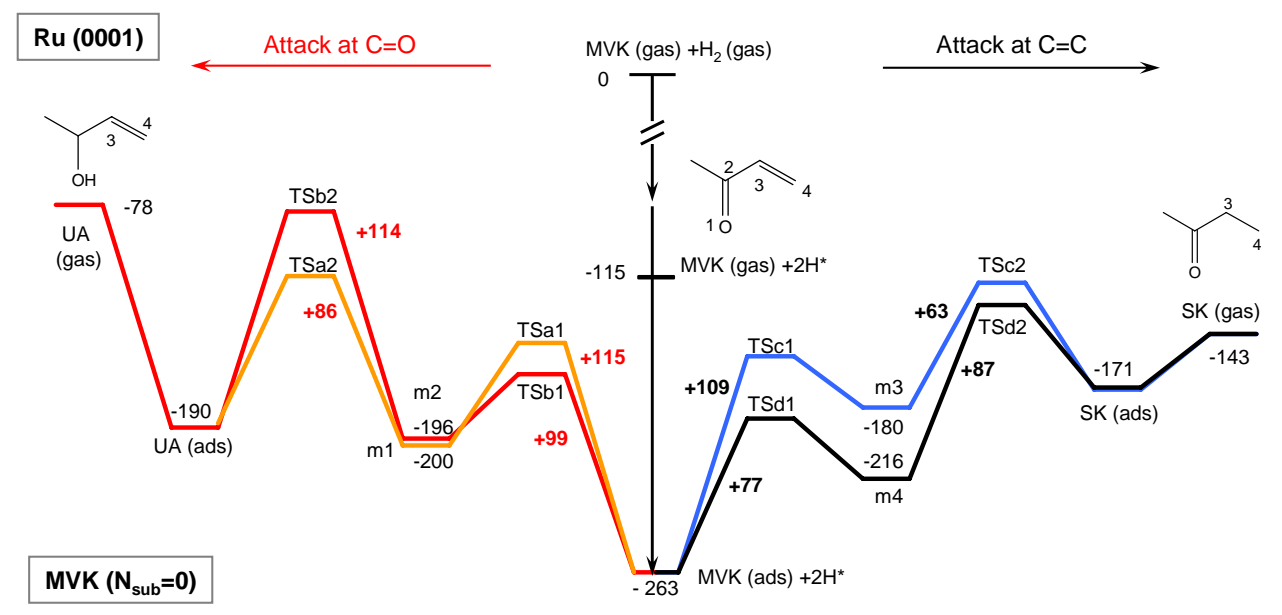

Figure 2.5 Energetic profiles for the hydrogenation of methyl vinyl ketone (MVK, substitution degree $=0$ ) on $\mathrm{Ru}(0001)$ surface. " $\mathrm{m}-\mathrm{x}$ " refers to the intermediates $\mathrm{m} 1-\mathrm{m} 4$ and "TS" refers to transition states.

The potential energy profiles for the four different paths involved in the hydrogenation of MVK to either UA or the SK are plotted together in Figure 2.5. The results indicate that the hydrogenation of the terminal $\mathrm{C}=\mathrm{C}$ is significantly easier than the hydrogenation of the ketone $\mathrm{C}=\mathrm{O}$ bond. This can be explained by the lower steric hindrance at the primary and secondary carbon centers of $\mathrm{C}=\mathrm{C}$ bond, over the more steric-hindered $\mathrm{C}=\mathrm{O}$ group which is comprised of tertiary carbon and primary oxygen sites. In the absence of the excessive steric hindrance at the carbonyl, there is a competition at the surface for the hydrogenation of the $\mathrm{C}=\mathrm{C}$ vs. $\mathrm{C}=\mathrm{O}$ groups, as suggested previously by Loffreda et al in the hydrogenation of unsaturated aldehydes [43].

The SK and UA products can either desorb from the surface or further hydrogenate to the saturated alcohol. The saturated ketone desorption is much easier than that for the unsaturated alcohol as the latter binds much more strongly to the surface $\left(-113 \mathrm{~kJ} \mathrm{~mol}^{-1}\right.$, Table 2.3$)$ than SK $\left(-28 \mathrm{~kJ} \mathrm{~mol}^{-1}\right)$. This is presumably the result of the much stronger steric repulsion between the tertiary carbon of the ketone and the Ru surface verses that of the primary carbon center on the terminal olefin and the Ru surface, as well as the differences between the bond strengths of the terminal $\mathrm{OH}$ and terminal $\mathrm{CH}_{2}$ groups with $\mathrm{Ru}$. The lower energy for desorption of the SK compared to the UA suggests that the UA would have a longer residence time on the surface and would therefore be more likely to be further hydrogenated to the saturated alcohol (SA). In addition, once the $\mathrm{C}=\mathrm{C}$ bonds of MVK are hydrogenated to the SK, significant amounts of the intermediate SK could be subsequently hydrogenated to the SA because the activation barriers for $\mathrm{C}=\mathrm{O}$ hydrogenation are not unreasonably high, which is consistent with the experimental observations of MVK hydrogenation on $\mathrm{Ru} / \mathrm{C}$ catalyst [13]. 
Table 2.3 DFT calculated adsorption energiers $\left(\mathrm{kJ} \mathrm{mol}^{-1}\right)$ and structural properties (bond lengths in $\AA$ ) for the most favorable adsorption modes for the reactants, intermediates and products of the hydrogenations of four unsaturated ketones over $\operatorname{Ru}(0001)$.

\begin{tabular}{|c|c|c|c|c|c|c|c|c|c|c|}
\hline \multicolumn{2}{|c|}{ Species } & \multirow{2}{*}{$\begin{array}{l}\Delta E_{a d s} \\
-148\end{array}$} & \multirow{2}{*}{$\frac{\text { Mode }}{\eta^{4} \text { cis }}$} & \multirow{2}{*}{$\begin{array}{l}\mathrm{O}-\mathrm{C}^{2} \\
1.37\end{array}$} & \multirow{2}{*}{$\frac{C^{2}-C^{3}}{1.42}$} & \multirow{2}{*}{$\frac{C^{3}-C^{4}}{1.45}$} & \multirow{2}{*}{$\frac{\mathrm{O}-\mathrm{Ru}}{2.21,2.25}$} & \multirow{2}{*}{$\frac{\mathrm{C}^{2}-\mathrm{Ru}}{2.28}$} & \multirow{2}{*}{$\frac{\mathrm{C}^{3}-\mathrm{Ru}}{2.38}$} & \multirow{2}{*}{$\frac{\mathrm{C}^{4}-\mathrm{Ru}}{2.16}$} \\
\hline \multirow{4}{*}{$\begin{array}{l}\text { Unsaturated } \\
\text { ketone }\end{array}$} & MVK & & & & & & & & & \\
\hline & IMK & -130 & $\eta^{4}$ cis & 1.38 & 1.43 & 1.46 & $2.23,2.23$ & 2.29 & 2.46 & 2.17 \\
\hline & MPO & -113 & $\eta^{4}$ cis & 1.38 & 1.43 & 1.46 & $2.30,2.17$ & 2.27 & 2.43 & 2.19 \\
\hline & DPO & -79 & $\eta^{4}$ cis & 1.39 & 1.44 & 1.49 & $2.24,2.17$ & 2.25 & 2.40 & 2.23 \\
\hline \multirow{4}{*}{$\mathrm{m} 1$} & m1-MVK & -177 & $\eta^{4}$ cis & 1.44 & 1.42 & 1.46 & 2.32 & 2.22 & 2.22 & $2.41,2.23$ \\
\hline & m1-IMK & -170 & $\eta^{4}$ cis & 1.44 & 1.43 & 1.47 & 2.33 & 2.23 & 2.23 & $2.43,2.24$ \\
\hline & m1-MPO & -150 & $\eta^{3}$ cis & 1.46 & 1.44 & 1.45 & 2.25 & 2.19 & - & 2.21 \\
\hline & m1-DPO & -122 & $\eta^{3}$ cis & 1.47 & 1.45 & 1.47 & 2.22 & 2.18 & - & 2.24 \\
\hline \multirow{4}{*}{$\mathrm{m} 2$} & m2-MVK & -300 & $\pi_{\mathrm{CC}} \eta^{2}(\mathrm{O})$ & 1.45 & 1.52 & 1.42 & $2.36,2.08$ & - & 2.23 & 2.22 \\
\hline & m2-IMK & -300 & $\pi_{\mathrm{CC}} \eta^{2}(\mathrm{O})$ & 1.45 & 1.54 & 1.44 & $2.14,2.15$ & - & 2.22 & 2.22 \\
\hline & m2-MPO & -282 & $\pi_{\mathrm{CC}} \eta^{2}(\mathrm{O})$ & 1.44 & 1.54 & 1.43 & $2.13,2.17$ & - & 2.25 & 2.23 \\
\hline & m2-DPO & -250 & $\pi_{\mathrm{CC}} \eta^{2}(\mathrm{O})$ & 1.45 & 1.54 & 1.43 & $2.14,2.19$ & - & 2.27 & 2.26 \\
\hline \multirow{4}{*}{$\mathrm{m} 3$} & m3-MVK & -195 & $\eta^{3}$ cis & 1.41 & 1.52 & 1.51 & $2.20,2.18$ & 2.18 & - & 2.15 \\
\hline & m3-IMK & -188 & $\eta^{3}$ cis & 1.40 & 1.54 & 1.52 & $2.26,2.20$ & 2.18 & - & 2.15 \\
\hline & m3-MPO & -155 & $\eta^{3}$ cis & 1.41 & 1.54 & 1.53 & $2.32,2.11$ & 2.19 & - & 2.17 \\
\hline & m3-DPO & -119 & $\eta^{3}$ cis & 1.42 & 1.54 & 1.55 & $2.25,2.16$ & 2.18 & - & 2.24 \\
\hline \multirow{4}{*}{$\mathrm{m} 4$} & m4-MVK & -178 & $\pi_{\mathrm{CC}} \eta^{2}(\mathrm{O})$ & 1.37 & 1.43 & 1.50 & $2.20,2.32$ & 2.30 & 2.25 & - \\
\hline & m4-IMK & -163 & $\pi_{\mathrm{CC}} \eta^{2}(\mathrm{O})$ & 1.37 & 1.43 & 1.51 & $2.20,2.33$ & 2.30 & 2.29 & - \\
\hline & m4-MPO & -142 & $\pi_{\mathrm{CC}} \eta^{2}(\mathrm{O})$ & 1.37 & 1.43 & 1.51 & $2.24,2.28$ & 2.32 & 2.29 & - \\
\hline & m4-DPO & -117 & $\pi_{\mathrm{CC}} \eta^{2}(\mathrm{O})$ & 1.38 & 1.43 & 1.54 & $2.24,2.27$ & 2.30 & 2.33 & - \\
\hline \multirow{4}{*}{$\begin{array}{l}\text { Unsaturated } \\
\text { alcohol }\end{array}$} & UA-MVK & -113 & $\pi_{\mathrm{CC}} \eta^{1}(\mathrm{O})$ & 1.46 & 1.51 & 1.44 & 2.26 & - & 2.16 & 2.22 \\
\hline & UA-IMK & -79 & $\pi_{\mathrm{CC}} \eta^{1}(\mathrm{O})$ & 1.48 & 1.54 & 1.44 & 2.26 & - & 2.23 & 2.20 \\
\hline & UA-MPO & -78 & $\pi_{\mathrm{CC}} \eta^{1}(\mathrm{O})$ & 1.46 & 1.52 & 1.43 & 2.24 & - & 2.25 & 2.23 \\
\hline & UA-DPO & -41 & $\pi_{\mathrm{CC}} \eta^{1}(\mathrm{O})$ & 1.46 & 1.53 & 1.44 & 2.24 & - & 2.24 & 2.28 \\
\hline \multirow{4}{*}{$\begin{array}{l}\text { Saturated } \\
\text { ketone }\end{array}$} & SK-MVK & -28 & $\eta^{2}(\mathrm{O}) \eta^{1}(\mathrm{C})$ & 1.42 & 1.53 & 1.51 & $2.19,2.22$ & 2.19 & - & - \\
\hline & SK-IMK & -18 & $\eta^{2}(\mathrm{O}) \eta^{1}(\mathrm{C})$ & 1.42 & 1.54 & 1.52 & $2.16,2.21$ & 2.22 & - & - \\
\hline & SK-MPO & -22 & $\eta^{2}(\mathrm{O}) \eta^{1}(\mathrm{C})$ & 1.42 & 1.55 & 1.53 & $2.16,2.21$ & 2.22 & - & - \\
\hline & SK-DPO & -8 & $\eta^{2}(\mathrm{O}) \eta^{1}(\mathrm{C})$ & 1.43 & 1.56 & 1.54 & $2.16,2.22$ & 2.22 & - & - \\
\hline
\end{tabular}


Table 2.4 DFT calculated activation barriers $\left(E_{a c t}, \mathrm{~kJ} \mathrm{~mol}^{-1}\right)$, reaction energies $\left(E_{r x n}, \mathrm{~kJ} \mathrm{~mol}^{-1}\right)$ and geometric parameters $(\AA)$ of transition states of the elementary steps in hydrogenations of four unsaturated ketones on $\mathrm{Ru}(0001)$. "A-H" in the first row refers to the atom to be hydrogenated (carbon or oxygen).

\begin{tabular}{|c|c|c|c|c|c|c|c|c|c|c|c|c|}
\hline & Paths & $E_{a c t}$ & $E_{r x n}$ & A-H & $\mathrm{H}-\mathrm{Ru}$ & $\mathrm{O}-\mathrm{C}^{2}$ & $C^{2}-C^{3}$ & $C^{3}-C^{4}$ & $\mathrm{O}-\mathrm{Ru}$ & $\mathrm{C}^{2}-\mathrm{Ru}$ & $\mathrm{C}^{3}-\mathrm{Ru}$ & $\mathrm{C}^{4}-\mathrm{Ru}$ \\
\hline \multirow{4}{*}{ a1 } & MVK & 115 & 63 & 1.36 & 1.90 & 1.37 & 1.41 & 1.46 & 2.26 & 2.45 & 2.27 & $2.41,2.22$ \\
\hline & IMK & 118 & 64 & 1.37 & 1.86 & 1.38 & 1.43 & 1.47 & 2.24 & 2.37 & 2.30 & $2.45,2.23$ \\
\hline & MPO & 120 & 63 & 1.37 & 1.81 & 1.40 & 1.45 & 1.45 & 2.18 & 2.29 & 2.62 & 2.22 \\
\hline & DPO & 123 & 75 & 1.37 & 1.75 & 1.41 & 1.45 & 1.47 & 2.18 & 2.26 & 2.54 & 2.23 \\
\hline \multirow{4}{*}{$\mathrm{b} 1$} & MVK & 99 & 67 & 1.54 & 1.68 & 1.36 & 1.49 & 1.42 & 2.09 & 2.64 & 2.33 & 2.20 \\
\hline & IMK & 77 & 39 & 1.65 & 1.70 & 1.31 & 1.49 & 1.44 & 2.07 & 2.75 & 2.21 & 2.19 \\
\hline & MPO & 62 & 31 & 1.74 & 1.69 & 1.31 & 1.49 & 1.44 & 2.08 & 2.80 & 2.23 & 2.20 \\
\hline & DPO & 58 & 33 & 1.74 & 1.69 & 1.31 & 1.49 & 1.45 & 2.08 & 2.78 & 2.27 & 2.24 \\
\hline \multirow{4}{*}{$\mathrm{c} 1$} & MVK & 109 & 83 & 1.26 & 2.00 & 1.37 & 1.52 & 1.50 & $2.27,2.20$ & 2.19 & 2.54 & 2.16 \\
\hline & IMK & 119 & 87 & 1.40 & 1.90 & 1.37 & 1.50 & 1.50 & $2.22,2.25$ & 2.20 & 2.62 & 2.17 \\
\hline & MPO & 118 & 85 & 1.38 & 1.93 & 1.37 & 1.53 & 1.52 & 2.20 & 2.24 & 2.70 & 2.18 \\
\hline & DPO & 118 & 71 & 1.40 & 1.95 & 1.37 & 1.53 & 1.53 & $2.29,2.17$ & 2.20 & 2.66 & 2.22 \\
\hline \multirow{4}{*}{ d1 } & MVK & 77 & 47 & 1.52 & 1.66 & 1.37 & 1.44 & 1.49 & $2.25,2.23$ & 2.26 & 2.33 & 2.27 \\
\hline & IMK & 78 & 47 & 1.52 & 1.66 & 1.37 & 1.43 & 1.48 & $2.27,2.23$ & 2.25 & 2.31 & 2.27 \\
\hline & MPO & 86 & 48 & 1.59 & 1.63 & 1.37 & 1.44 & 1.49 & $2.27,2.22$ & 2.26 & 2.31 & 2.32 \\
\hline & DPO & 89 & 46 & 1.58 & 1.63 & 1.38 & 1.44 & 1.51 & $2.26,2.22$ & 2.28 & 2.32 & 2.40 \\
\hline \multirow{4}{*}{ a2 } & MVK & 86 & 12 & 1.49 & 1.68 & 1.46 & 1.49 & 1.43 & 2.29 & 2.33 & 2.23 & 2.19 \\
\hline & IMK & 89 & 38 & 1.49 & 1.71 & 1.45 & 1.48 & 1.42 & 2.29 & 2.36 & 2.33 & 2.22 \\
\hline & MPO & 88 & 17 & 1.47 & 1.69 & 1.46 & 1.49 & 1.43 & 2.26 & 2.38 & 2.35 & 2.20 \\
\hline & DPO & 75 & 2 & 1.49 & 1.68 & 1.45 & 1.50 & 1.46 & 2.23 & 2.40 & 2.32 & 2.22 \\
\hline \multirow{4}{*}{ b2 } & MVK & 114 & 5 & 1.40 & 1.98 & 1.42 & 1.51 & 1.42 & 2.13 & - & 2.20 & 2.24 \\
\hline & IMK & 123 & 64 & 1.45 & 1.90 & 1.44 & 1.55 & 1.45 & 2.12 & - & 2.20 & 2.19 \\
\hline & MPO & 122 & 48 & 1.43 & 1.91 & 1.44 & 1.55 & 1.45 & 2.12 & - & 2.27 & 2.22 \\
\hline & DPO & 120 & 43 & 1.44 & 1.87 & 1.43 & 1.55 & 1.44 & 2.11 & - & 2.30 & 2.26 \\
\hline \multirow{4}{*}{ c2 } & MVK & 63 & 9 & 1.58 & 1.66 & 1.42 & 1.53 & 1.51 & $2.25,2.18$ & 2.17 & - & 2.27 \\
\hline & IMK & 63 & 15 & 1.56 & 1.66 & 1.41 & 1.54 & 1.52 & $2.24,2.20$ & 2.17 & - & 2.26 \\
\hline & MPO & 70 & 14 & 1.55 & 1.69 & 1.42 & 1.54 & 1.54 & $2.25,2.16$ & 2.17 & - & 2.36 \\
\hline & DPO & 77 & 3 & 1.56 & 1.66 & 1.42 & 1.54 & 1.55 & $2.24,2.16$ & 2.16 & - & 2.42 \\
\hline \multirow{4}{*}{$\mathrm{d} 2$} & MVK & 87 & 46 & 1.49 & 1.80 & 1.37 & 1.48 & 1.52 & $2.23,2.28$ & 2.23 & 2.43 & - \\
\hline & IMK & 87 & 55 & 1.49 & 1.76 & 1.38 & 1.46 & 1.52 & $2.19,2.31$ & 2.26 & 2.42 & - \\
\hline & MPO & 86 & 50 & 1.51 & 1.79 & 1.38 & 1.46 & 1.53 & $2.16,2.38$ & 2.24 & 2.46 & - \\
\hline & DPO & 83 & 27 & 1.52 & 1.78 & 1.38 & 1.46 & 1.55 & $2.19,2.34$ & 2.26 & 2.42 & - \\
\hline
\end{tabular}




\section{2) Substitution Effects on the Adsorption of Reactants and Products}

The $\alpha, \beta$-unsaturated ketones can have various adsorption modes on metal surfaces as each $\mathrm{C}=\mathrm{C}$ and $\mathrm{C}=\mathrm{O}$, can interact with the surface separately or in combination. The binding modes that occur through both $\mathrm{C}=\mathrm{C}$ and $\mathrm{C}=\mathrm{O}$ bonds at multi-coordinated sites (e.g., $\eta^{3}, \eta^{4}$ ) were found to be the most stable modes of adsorption at low coverage at a wide range of temperatures $[34,35,81]$. The adsorption energies for all of the ketone reactants as well as the corresponding intermediates and products on $\mathrm{Ru}(0001)$ surface are reported in Table 2.3 and in Figure 2.6. The later clearly shows a decrease in the adsorption energy as we weaken the interaction with the $\mathrm{C}=\mathrm{C}$ bond via the addition of alkyl substituents. The increasing substitution degree $\left(\boldsymbol{N}_{\text {sub }}\right)$ affects the binding energy near-linearly and each methyl substituent appears to weaken the adsorption energy by $20 \mathrm{~kJ} \mathrm{~mol}^{-1}$. The most stable adsorption mode for an unsaturated ketone (at 1/9ML coverage) was found to be a planar $\eta^{4}$-cis configuration where both the carbonyl and $\mathrm{C}=\mathrm{C}$ bonds are orientated parallel to the surface and interact with $\mathrm{Ru}$ atoms (Figure 2.6a). This results in a considerable elongation of both double bonds upon adsorption from gas phase. To compensate these elongations, the $\mathrm{C}^{2}-\mathrm{C}^{3}$ that connects these two double bonds was decreased by $0.06 \AA$ and exhibits $\pi$ bond character, where both the $C^{2}$ and $C^{3}$ centers coordinate to the same Ru surface center. The repulsive interactions between the methyl substituent on the $\mathrm{C}=\mathrm{O}$ bond and along with the strong attractive interaction between the oxygen and the $\mathrm{Ru}$ sites drives a re-hybridization of carbon $\mathrm{C}^{2}$ from planar $\mathrm{sp}^{2}$ hybridization to tetrahedral $\mathrm{sp}^{3}$ hybridization. This rehybridization allows both the carbon and oxygen atoms to form strong covalent bonds with metal surface and reorients the methyl substituent away from the surface. The hydrogen addition reactions were studied from this stable $\eta^{4}$ state to which the intrinsic barriers of elementary steps were referenced.

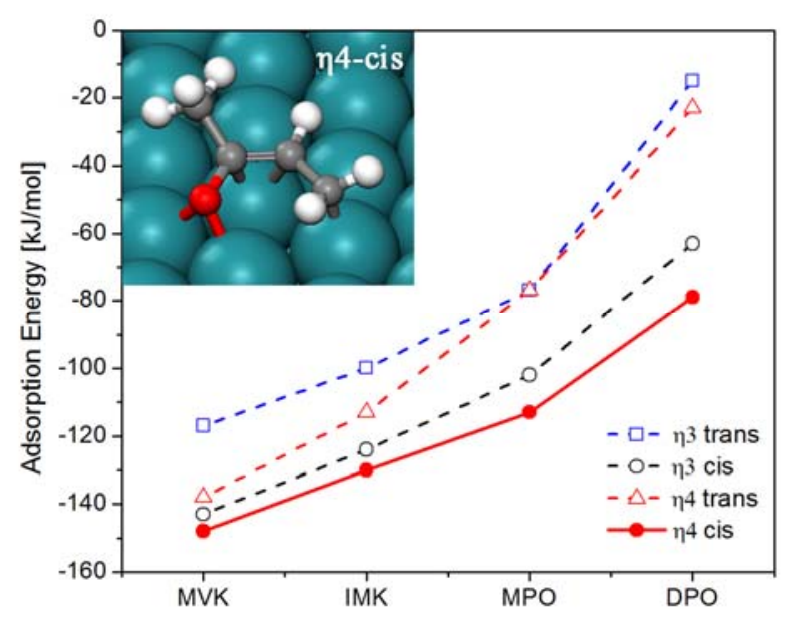

(a)

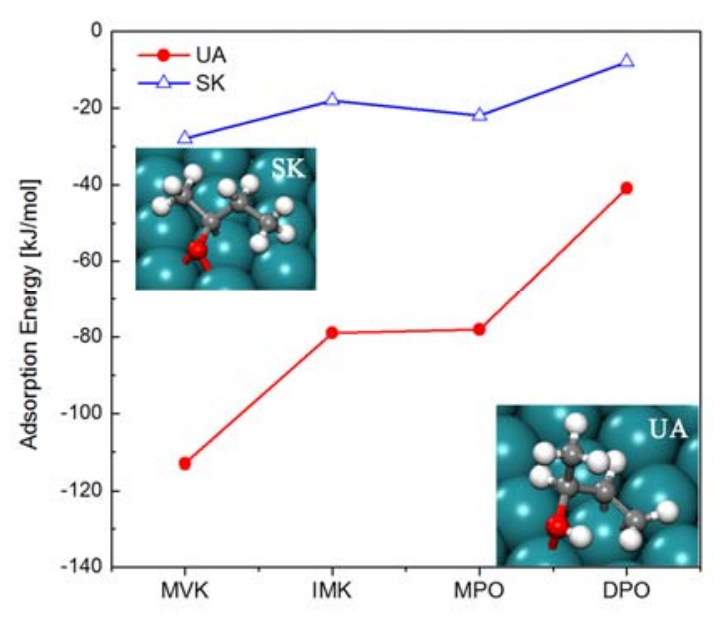

(b)

Figure 2.6 The change in the adsorption energies of (a) different adsorption modes of unsaturated ketones and (b) unsaturated alcohols (UA) and saturated ketones (SK) on $\mathrm{Ru}(0001)$, with increasing the degree of substitution at $\mathrm{C}=\mathrm{C}$ : 0 for MVK, 1 for IMK, 2 for MPO and 3 for DPO. Adsorption geometries in the inserts are shown by MVK as a representative. O-red, C-dark grey, H-white. 
The adsorption of the partially hydrogenated intermediates and products on $\mathrm{Ru}$ were also found to be weakened by substitution effects, which agrees well with previous theoretical results of acrolein and other unsaturated aldehydes $[36,39]$. As shown in Table 2.3, each methyl substituent weakens the adsorption energy of the intermediates by $10-35 \mathrm{~kJ} \mathrm{~mol}^{-1}$. The changes in adsorption energies will thus affect the reaction energetics as will be shown later. The alkoxide intermediate, m2, adsorbs most strongly to the surface (over $100 \mathrm{~kJ} / \mathrm{mol}$ stronger than the other three intermediates), due to the fact that these species are least stable in gas phase and thus most reactive. The hydroxyalkyl intermediates $\mathrm{m} 1$ and alkyl $\mathrm{m} 4$ are more stable in gas phase as a result of their ability to delocalize the free radical over conjugated $\pi$ system, and thus bind less strongly than the alkoxide. The adsorption of the unsaturated alcohol products were found to be more sensitive to the substitution effects than the alternative saturated ketone products (Figure 2.6b), as the $\pi_{\mathrm{CC}}$ interaction between the $\mathrm{C}=\mathrm{C}$ bond of $\mathrm{UA}$ and the surface can be significantly influenced by the presence of methyl substituents. The interaction of saturated ketone with $\mathrm{Ru}$ surface occurs solely via the carbonyl group and is thus not significantly affected by the presence of substituents on the $\mathrm{C}=\mathrm{C}$ bond (Figure $2.6 \mathrm{~b}$ ).

Atomic hydrogen was calculated to have the strongest binding at the 3-fold hollow $f c c$ site on $\mathrm{Ru}(0001)$. All three $\mathrm{H}-\mathrm{Ru}$ bonds was calculated to be $1.90 \AA$, with a binding energy of $-279 \mathrm{~kJ} \mathrm{~mol}^{-1}(-2.90 \mathrm{eV})$, which agree well with the previous theoretical studies on ruthenium [82]. All of the binding energies reported here refer to the adsorption of gas phase free radical intermediates onto Ru. For hydrogen these energy is referenced to the gas phase atomic hydrogen.

\section{3) Substitution Influence on the Hydrogenation of Unsaturated Ketones}

The DFT-calculated activation barriers and reaction energies for all four of the ketones examined are tabulated in Table 2.4. The initial hydrogenation paths are found to be endothermic for all the reactants on $\mathrm{Ru}(0001)$. This tends to occur on metal surfaces that bind atomic hydrogen very strongly. Surfaces that have weak metal$\mathrm{H}$ bonds such as $\mathrm{Os}(0001)$ and $\operatorname{Ir}(111)$ typically result in highly exothermic reactions involving the hydrogenation of carbonyl intermediates as the energy penalty that results from breaking the $\mathrm{M}-\mathrm{H}$ bond is much less [83]. The reaction energies for path $\mathrm{b} 1$ that hydrogenate $\mathrm{C}=\mathrm{O}$ bond to form the most stable intermediate alkoxide intermediate $\mathrm{m} 2$ were found to be the most sensitive to the changing hydrocarbon structures. The changing trend in reaction energies was found to be similar to the trend of barriers, which is consistent with the Brönsted-Evans-Polanyi (BEP) relationship that linearly relates the changes in activation barrier to the changes in the heat of reaction [84]. 
The degree of substitution $\left(\boldsymbol{N}_{\text {sub }}\right)$ at the olefin $\mathrm{C}=\mathrm{C}$ bond shows a notable influence on the energetics of the first hydrogenation step as it increases the activation barrier for hydrogen addition to the substituted carbon site of the $\mathrm{C}=\mathrm{C}$ bond while lowering the barrier for hydrogen addition to the carbon center of the carbonyl group (Table 2.4). The direct substitution of hydrogen atoms for a methyl substituent at the carbon site that is to be hydrogenated increased the barrier for hydrogenation by $10 \mathrm{~kJ} \mathrm{~mol}^{-1}$ per substituent (e.g., path $\mathrm{c} 1$ in Table 2.4). These substitutions also weakened the interaction and reduced steric hindrance at the neighboring carbon center of the carbonyl which promotes the addition of the first hydrogen to this site. The barrier to hydrogenate the carbon atom of $\mathrm{C}=\mathrm{O}$ bond was calculated to be $20 \mathrm{~kJ} \mathrm{~mol}^{-1}$ lower per substitutuent. As a result, the relative ordering of the activation barriers for adding the first hydrogen to each of the adsorbed ketone reactants are given in eq. (2.1) - (2.4):

$$
\begin{aligned}
\text { MVK: } & \mathrm{C}^{4} \text { path } \mathrm{d} 1<\mathrm{C}^{2}-(\mathrm{O}) \text { path } \mathrm{b} 1<\mathrm{C}^{3} \text { path } \mathrm{c} 1<\mathrm{O}^{1} \text { path } \mathrm{a} 1 \\
\text { IMK: } & \mathrm{C}^{4} \text { path } \mathrm{d} 1=\mathrm{C}^{2}-(\mathrm{O}) \text { path } \mathrm{b} 1<\mathrm{C}^{3} \text { path } \mathrm{c} 1=\mathrm{O}^{1} \text { path } \mathrm{a} 1 \\
\text { MPO: } & \mathrm{C}^{2}-(\mathrm{O}) \text { path } \mathrm{b} 1<\mathrm{C}^{4} \text { path } \mathrm{d} 1<\mathrm{C}^{3} \text { path } \mathrm{c} 1<\mathrm{O}^{1} \text { path a1 } \\
\text { DPO: } & \mathrm{C}^{2}-(\mathrm{O}) \text { path } \mathrm{b} 1<\mathrm{C}^{4} \text { path } \mathrm{d} 1<\mathrm{C}^{3} \text { path } \mathrm{c} 1<\mathrm{O}^{1} \text { path a1 }
\end{aligned}
$$

The first hydrogen preferentially reacts at the unsubstituted terminal carbon $\mathrm{C}^{4}$ for the hydrogenation of MVK $\left(\boldsymbol{N}_{\text {sub }}=0\right)$. The addition of a single methyl substituent at the internal $\mathrm{C}^{3}$ carbon atom to form IMK $\left(\boldsymbol{N}_{\text {sub }}=1\right)$ decreased the barrier to hydrogenate the neighboring $\mathrm{C}^{2}$ site of the $\mathrm{C}=\mathrm{O}$ bond by $22 \mathrm{~kJ} \mathrm{~mol}^{-1}$. As a result, both the alkoxide path (b1) and alkyl path (d1) become the most favorable routes for the initial hydrogenation (eq. 2.2). The addition of a second and third methyl substituents at the terminal $\mathrm{C}^{4}$ carbon to form MPO $\left(N_{\text {sub }}=2\right)$ and DPO $\left(N_{\text {sub }}=3\right)$, respectively reduced the barriers to hydrogenate $\mathrm{C}^{2}$ site of the carbonyl to $\sim 60 \mathrm{~kJ} \mathrm{~mol}^{-1}$ thus making the alkoxide path the most favorable as is shown in eq. (2.3) and (2.4).

The highest initial hydrogen addition activation barrier for all of the substituted unsaturated ketones examined always occurs for the at $\mathrm{O}^{1}$ oxygen of the carbonyl (path a1) as is shown in eqs. 2.1-2.4. This correlates with the previous theoretical results which show that hydrogen preferentially adds to the carbon rather than the oxygen in the hydrogenation of ketones over $\mathrm{Ru}(0001)$ [45]. Methyl substituents at the terminal $\mathrm{C}^{4}$ increase the barrier for adding the first hydrogen to this site, but the influence is not as significant as that on the two interior carbons (Table 2.4). This can be better understood by more carefully examining the structures of the transition states presented in Figure 2.7 - 2.9. The initial addition of hydrogen to either of the two interior carbon centers $\left(\mathrm{C}^{2}\right.$ and $\left.\mathrm{C}^{3}\right)$ significantly disrupts the conjugated $\pi$ resonance system that is delocalized over the $\mathrm{C}=\mathrm{C}-\mathrm{C}=\mathrm{O}$ backbone. This can either significantly stabilize (or destabilize) the transition state over that of the reactant and thus leads a considerably lower (or higher) activation barriers. The hydrogen attack at the terminal $\mathrm{O}^{1}$ or $\mathrm{C}^{4}$ centers, however, lead to only small structural changes to the $\mathrm{C}^{2}=\mathrm{O}$ or $\mathrm{C}^{3}=\mathrm{C}^{4}$ bonds and have much less of an effect on the activation barriers. 

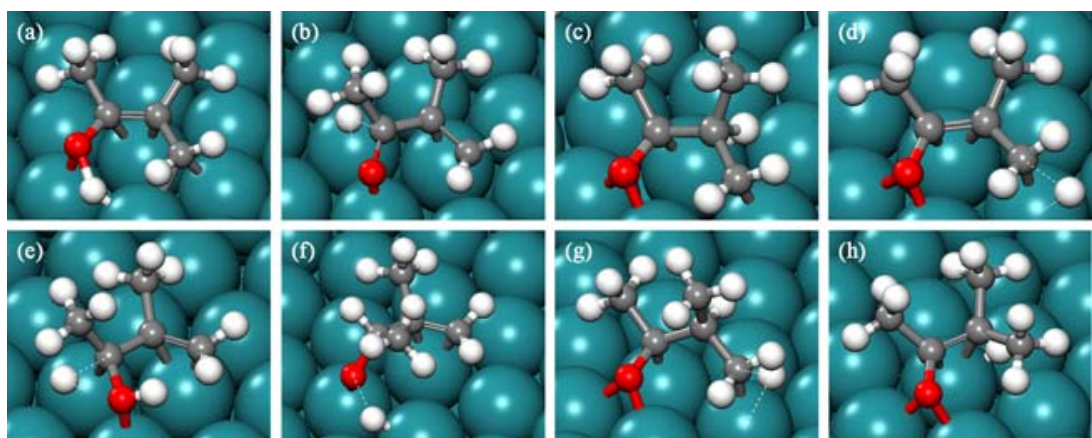

Figure 2.7 DFT-calculated transition state structures for (a) path a1; (b) path b1; (c) path c1; (d) path d1; (e) path a2; (f) path b2; (g) path c2; (h) path d2 during IMK hydrogenation on $\mathrm{Ru}(0001)$.

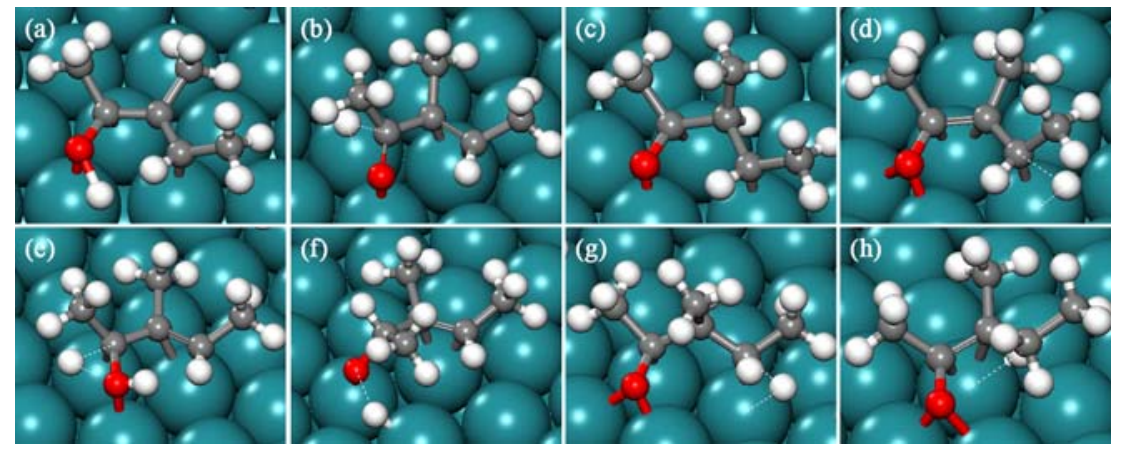

Figure 2.8 DFT-calculated transition state structures for (a) path a1; (b) path b1; (c) path c1; (d) path d1; (e) path a2; (f) path b2; (g) path c2; (h) path d2 during MPO hydrogenation on $\mathrm{Ru}(0001)$.

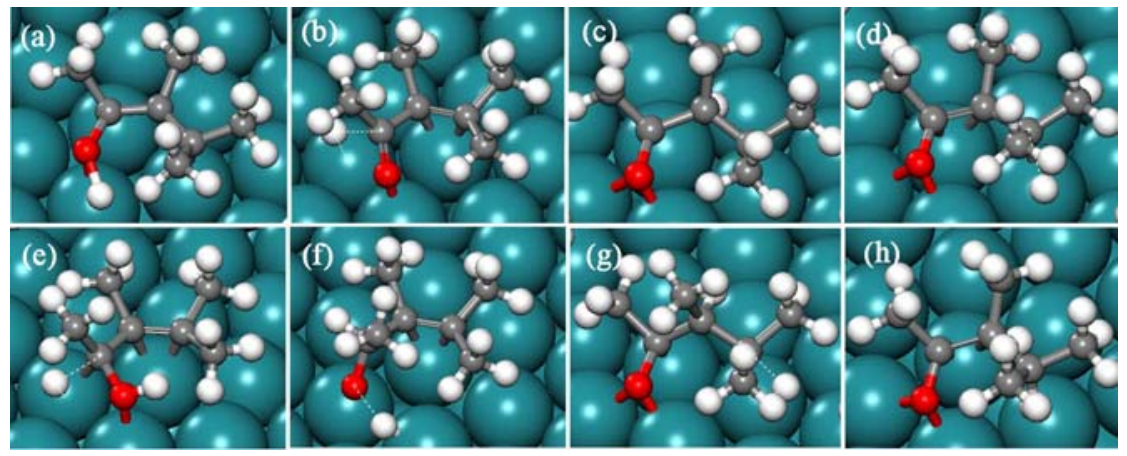

Figure 2.9 DFT-calculated transition state structures for (a) path a1; (b) path b1; (c) path c1; (d) path d1; (e) path a2; (f) path b2; (g) path c2; (h) path d2 during DPO hydrogenation on $\mathrm{Ru}(0001)$. 


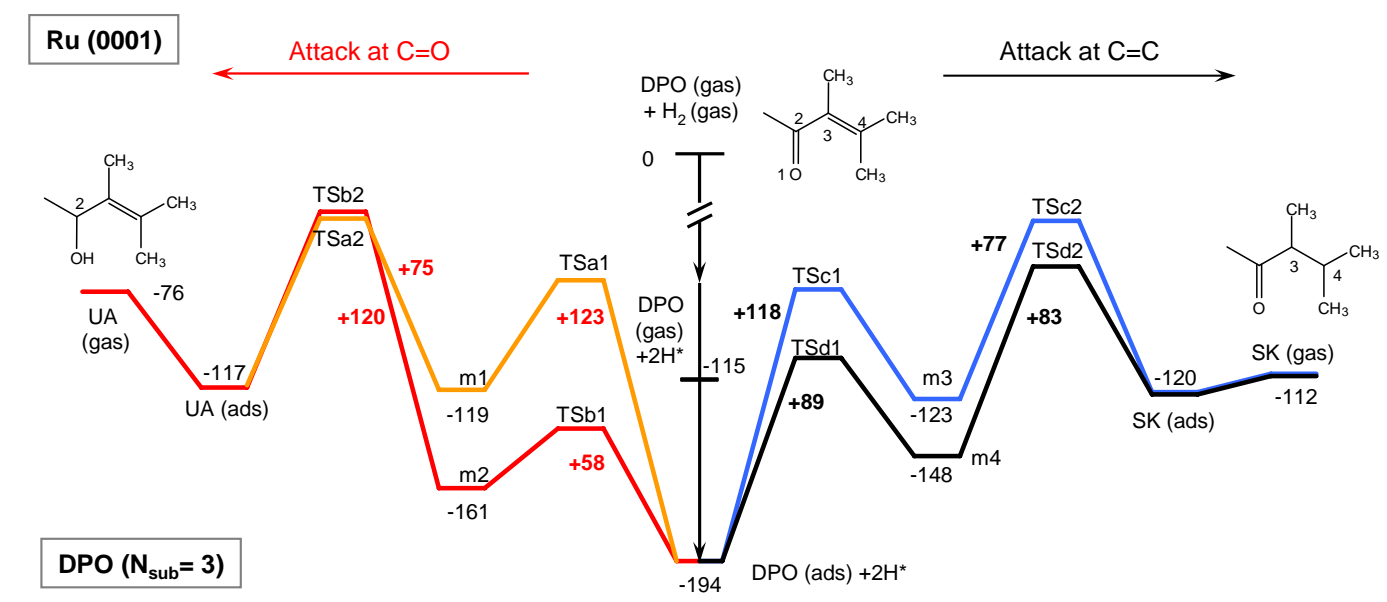

Figure 2.10 DFT-calculated reaction energetic profiles for the hydrogenations of 3,4-dimethyl-3-penten-2-one (DPO, substitution degree $=3)$ on the $\mathrm{Ru}(0001)$ surface. " $\mathrm{m}-\mathrm{x}$ " refers to the intermediates $\mathrm{m} 1-\mathrm{m} 4$ and "TS" refers to transition states.

Figure 2.10 shows the potential energy profiles for the hydrogenation of DPO $\left(\boldsymbol{N}_{\text {sub }}=3\right)$ over the $\operatorname{Ru}(0001)$ surface. Compared to MVK, the addition of the methyl substituents to the $\mathrm{C}=\mathrm{C}$ bond of DPO was found to considerably facilitate the hydrogenation of the carbon center of the $\mathrm{C}=\mathrm{O}$ bond and inhibit the hydrogen addition to the $\mathrm{C}=\mathrm{C}$ bond. The subsequent hydrogenation of the resulting alkoxide to form the unsaturated alcohol, however, is very difficult requiring activation barriers of least $120 \mathrm{~kJ} \mathrm{~mol}^{-1}$ to overcome the loss of the strong $\mathrm{Ru}-\mathrm{O}$ bond. The barriers of this step are not significantly influenced by the methyl substituent at the $\mathrm{C}=\mathrm{C}$ bond as the carbon backbone of the alkoxide is oriented well away from the Ru surface. In contrast, the subsequent hydrogenation of the $\mathrm{C}=\mathrm{C}$ bond $\left(\sim 80 \mathrm{~kJ} \mathrm{~mol}^{-1}\right)$ is significantly easier than addition to the oxygen of the alkoxide and thus leads to the production of saturated ketones as the major product over $\mathrm{Ru}(0001)$.

Theoretical results here suggest that although the substitution to $\mathrm{C}=\mathrm{C}$ bond lowers the barriers for the initial hydrogen addition to the $\mathrm{C}^{2}$ of the carbonyl, it does not lower the barrier for the more difficult second hydrogen addition step which requires breaking the strong $\mathrm{Ru}-\mathrm{O}$ bond. There is little influence of the barrier for hydrogenating the alkoxide structures regardless of the degree of carbon substitution as the carbon sites are oriented away from the surface. Increasing the degree of substitute on the $\mathrm{C}=\mathrm{C}$ sites therefore has little influence on the catalytic selectivity of unsaturated ketone hydrogenation. This rationalizes the experimental observations that the hydrogenation of $\alpha, \beta$-unsaturated ketones on supported $\mathrm{Ru} / \mathrm{C}$ catalysts shows very low or negligible selectivity to unsaturated alcohol at high conversions [13]. 


\section{4) Kinetics Analysis of the Hydrogenation}

The DFT calculated results were used to construct a simple microkinetic model to solve the simultaneous set of nonlinear differential equations with respect to time in order to simulate the surface coverage and analyze the controlling kinetics under working conditions. The reaction is thought to proceed via a Horiuti-Polanyi mechanism involving the adsorption of the unsaturated ketone and the dissociative adsorption of hydrogen followed by a series of sequential H-addition steps and the desorption of products [85] as is shown in steps 1-7:

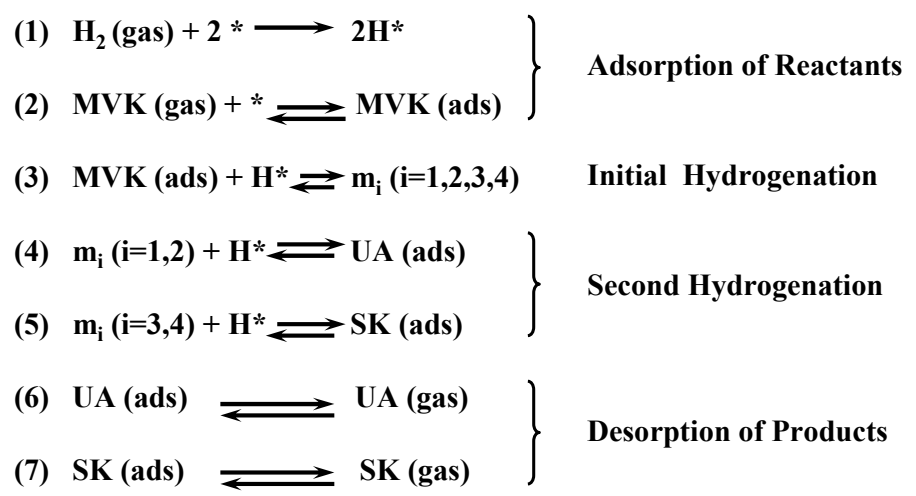

Steps 1 and 2 involve the adsorption of $\mathrm{H}_{2}$ and the unsaturated ketone, respectively. Step 3 corresponds to the first hydrogen addition steps on the $\mathrm{Ru}(0001)$ surface which lead to four partially hydrogenated intermediates, $\mathrm{m}_{\mathrm{i}}$. Steps 4 and 5 involve the subsequent addition of the second hydrogen atom to form the unsaturated alcohol or saturated ketone products which desorb in steps 6 and 7.

The kinetics simulations were carried out at constant temperature $(\mathrm{T}=350 \mathrm{~K})$ and constant partial pressures of MVK $\left(\mathrm{P}_{\mathrm{MVK}}=25\right.$ Torr $)$ and hydrogen $\left(\mathrm{P}_{\mathrm{H} 2}=500\right.$ Torr $)$, respectively. The rate constants $k$ used in the simulation were calculated from the activation barriers from our theoretical calculations by using the Arrhenius equation. The pre-exponential factors $A$ were set at $10^{13} \mathrm{~s}^{-1}$ for all the hydrogenation steps and at $10^{9} \mathrm{~s}^{-1}$ for the associative desorption of hydrogen [86]. The barriers for the adsorption are assumed to be negligible, and the sticking coefficients were used instead of the pre-exponential factors [87] with the adsorption rate was determined by:

$$
k_{H}=s_{0} \cdot P_{H_{2}} \cdot A s \cdot(2 \pi \cdot M w \cdot R T)^{-0.5}
$$

where $s_{0}, P_{H 2}, M w$, and $A s$ are the sticking coefficient, the partial pressure of species, the molecular weight of one molecule, and the surface area of the sites involved. The sticking coefficients $s_{0}$ of hydrogen and unsaturated ketone are chosen to be 0.1 and 1 , respectively, from previous theoretical studies [86]. Diffusion of hydrogen atom is considered very fast, that the time $t\left(\mathrm{H}^{*}\right.$ diffuse $)<t($ ads $)<<t$ (reaction). The influence of diffusion on the hydrogenation on surfaces such as $\mathrm{Ru}$ and $\mathrm{Pt}$ has been previously examined and it was found that it did not significantly influence the results [88]. 
The simulated surface coverages for the hydrogenation of MVK $\left(\boldsymbol{N}_{\text {sub }}=0\right)$ and DPO $\left(\boldsymbol{N}_{\text {sub }}=3\right)$ are summarized in Table 2.5 and Figure 2.11. In the hydrogenation of MVK, the alkyl intermediate, $\mathrm{m} 4$, has the highest coverage among the four partially-hydrogenated intermediates at the steady state (Figure 2.11a). This is consistent with the very rapid addition of the first hydrogen to the un-substituted terminal carbon of MVK that results due to the low barrier of $77 \mathrm{~kJ} \mathrm{~mol}^{-1}$.

Table 2.5 Simulated steady-state surface coverages of the hydrogenations of MVK $\left(\boldsymbol{N}_{\text {sub }}=0\right)$ and DPO $\left(\boldsymbol{N}_{\text {sub }}=3\right)$ at $350 \mathrm{~K}$. The partial pressure $\mathrm{P}_{\mathrm{MVK}}=25$ Torr and $\mathrm{P}_{\mathrm{H} 2}=500$ Torr.

\begin{tabular}{c|ccc}
\hline Coverage (ML) & $\mathrm{H}^{*}$ & alkoxy $m 2$ & alkyl $m 4$ \\
\hline MVK & 0.40 & 0.003 & 0.195 \\
DPO & 0.34 & 0.28 & 0.014 \\
\hline
\end{tabular}

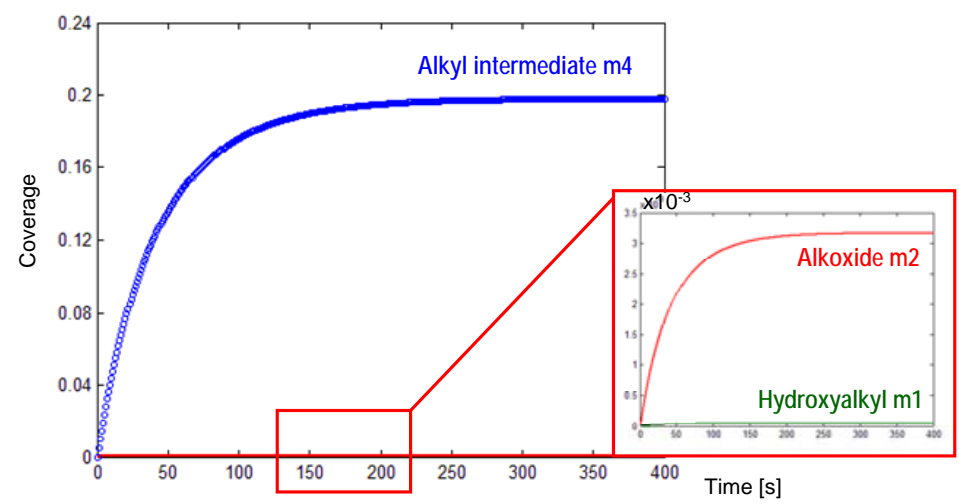

(a)

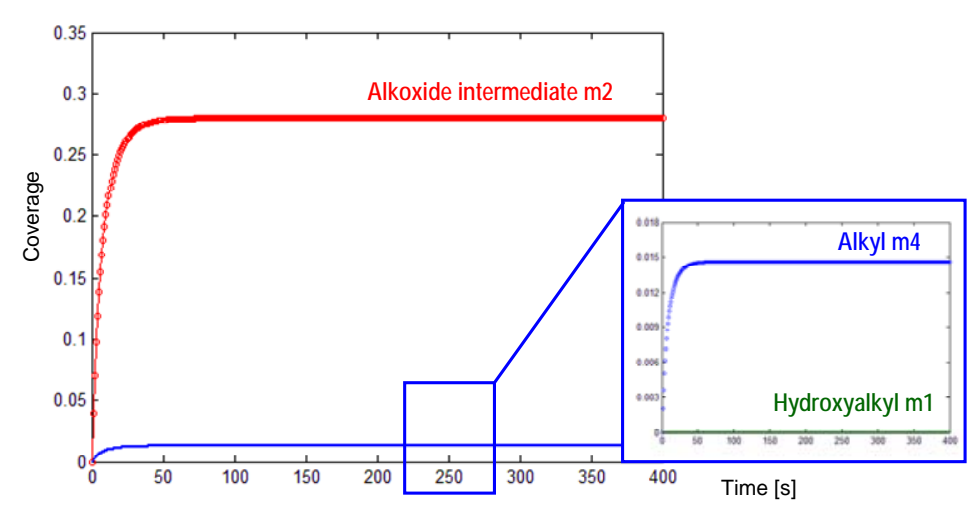

(b)

Figure 2.11 Plots of the surface coverages of different intermediates on $\mathrm{Ru}(0001)$ vs. time, via the kinetics analysis at $350 \mathrm{~K}$ for the hydrogenation of (a) MVK $\left(N_{\text {sub }}=0\right)$; (b) DPO $\left(N_{\text {sub }}=3\right)$. 
While the substitutions at the $\mathrm{C}=\mathrm{C}$ bond of DPO significantly increase the rate of formation of the alkoxide intermediate (seven orders of magnitude greater as compared to that of MVK, estimated at $350 \mathrm{~K}$ ), there is little influence on the rate of alkoxide hydrogenation which is always rather slow at ranging between $\sim 10^{-5}-10^{-4} \mathrm{~s}^{-1}$ at $350 \mathrm{~K}$. The rapid formation and the fairly slow consumption of the strongly bound alkoxide intermediate result in the rapid accumulation of alkoxide on the surface (Figure 2.11b). During the reaction of DPO, the alkoxide intermediate (m2) and the hydrogen are present as the main species on the Ru surface with coverages of $0.28 \mathrm{ML}$ and $0.34 \mathrm{ML}$, respectively (Table 2.5 ). The production of unsaturated alcohol is limited by the hydrogenation of the alkoxide. The results are consistent with previous in situ FTIR studies of crotonaldehyde hydrogenation which detect the unsaturated alkoxide intermediate whose hydrogenation rate controls the overall rate of reaction [89]. The saturated ketone species that form rapidly desorb from the surface and do not accumulate whereas the unsaturated alcohols that form have much longer residence times on the surface as they held much more strongly than the saturated ketones. As a result, the unsaturated alcohols can further react to form saturated alcohols. As such the selective production of unsaturated alcohols requires a strong enough interaction with the surface to favor its formation but a weak enough interaction with the Ru allowing it to be readily desorbed. While the addition of methyl substituents weakly favors the initial addition of hydrogen to the carbon center of the carbonyl, they are unable to influence the strong Ru-O interaction in the alkoxide. The selective hydrogenation of the unsaturated ketones could likely be achieved by substituting the unsaturated $\mathrm{C}=\mathrm{C}$ bond and moving to a less oxophilic metal.

\section{CONCLUSIONS}

The reactivity trends for the hydrogenations of a series of model $\alpha, \beta$-unsaturated ketones including methyl vinyl ketone (MVK), isopropenyl methyl ketone (IMK), 3-methyl-3-penten-2-one (MPO) and 3,4-dimethyl-3penten-2-one (DPO) were analyzed through periodic DFT calculations over model $\mathrm{Ru}(0001)$ surface. The hydrogenation of the terminal $\mathrm{C}=\mathrm{C}$ bond of $\operatorname{MVK}\left(N_{s u b}=0\right)$ is significantly easier than the hydrogenation of the $\mathrm{C}=\mathrm{O}$ bond. This is due to the considerably lower steric hindrance at the primary-secondary $\mathrm{C}=\mathrm{C}$ bond versus the more sterically-crowded $\mathrm{C}$ center of $\mathrm{C}=\mathrm{O}$ bond and, in addition, the relative ease of hydrogenating the weaker $\mathrm{Ru}-\mathrm{CH}_{2} \mathrm{R}$ bonds over the strong Ru-OR bonds. Both the primary unsaturated alcohol and saturated ketone products can desorb from the surface or be further hydrogenated into saturated alcohol (SA). The desorption of the saturated ketone is significantly easier than that desorption of the unsaturated alcohol due to the much stronger binding energy of the unsaturated alcohol than the saturated ketone to $\mathrm{Ru}$. This is consistent with the experimental results that no UA and a small amount of SA (2\%) are obtained in the hydrogenation of MVK over $\mathrm{Ru} / \mathrm{C}$ at $72 \%$ conversion [13]. 
Substituents at the olefin $\mathrm{C}=\mathrm{C}$ bond of an unsaturated ketone weaken the adsorption energy of the reactant by $\sim 20 \mathrm{~kJ} \mathrm{~mol}^{-1}$ per methyl substituent and shows notable increases the rates for the initial hydrogen addition to the carbon of the carbonyl over hydrogen addition to the $\mathrm{C}=\mathrm{C}$ bond. It increases the barrier to add hydrogen to the $\mathrm{C}=\mathrm{C}$ bond the substituted carbon sites of the $\mathrm{C}=\mathrm{C}$ bond by $10 \mathrm{~kJ} \mathrm{~mol}^{-1}$ while lowering the barrier by $20 \mathrm{~kJ}$ $\mathrm{mol}^{-1}$ to add to the $\mathrm{C}$ of the carbonyl. The subsequent hydrogenation of strongly bound alkoxide intermedate, however, require overcoming a barrier of $\sim 120 \mathrm{~kJ} \mathrm{~mol}^{-1}$ and is not significantly influenced by the methyl substituent at the $\mathrm{C}=\mathrm{C}$ bond. In contrast, the subsequent hydrogenation of the $\mathrm{C}=\mathrm{C}$ bond is easier $\left(\sim 80 \mathrm{~kJ} \mathrm{~mol}^{-1}\right)$ and leads to the production of saturated ketones as the major product on the Ru surface.

Microkinetic simulations carried out at $350 \mathrm{~K}$ and constant partial pressures of unsaturated ketone and hydrogen show that increasing the substitution of $\mathrm{C}=\mathrm{C}$ bond in an $\alpha, \beta$-unsaturated ketone decreases the rate of $\mathrm{C}=\mathrm{C}$ bond hydrogenation and markedly increases the rate of alkoxide formation on $\mathrm{Ru}$. This, however, is not enough to fully reverse the changes in selectivity as it does not appreciably influence the consumption of alkoxide intermediate, which is important in production of desired unsaturated alcohol. Thus, the hydrogenation of the $\mathrm{C}=\mathrm{C}$ bond is still more favorable than that of the $\mathrm{C}=\mathrm{O}$ bond on $\mathrm{Ru}$, which rationalizes the observed low selectivity to unsaturated alcohols in experiments. The subsequent hydrogenation of the alkoxide to form the unsaturated alcohol is inhibited by the strong $\mathrm{Ru}-\mathrm{O}$ bond that forms. The results suggest that increasing the methyl substituents to the $\mathrm{C}=\mathrm{C}$ bond and moving to a less oxophillic metal could significantly increase the selectivity to the unsaturated alcohol. 


\section{Chapter 3 - Co-operative Effects of the Metal Type and Reactant Structure on the Catalytic Hydrogenations of Complex Unsaturated Ketones}

\subsection{Hydrogenation of Benzalacetone over Supported Pt and Ru Catalysts}

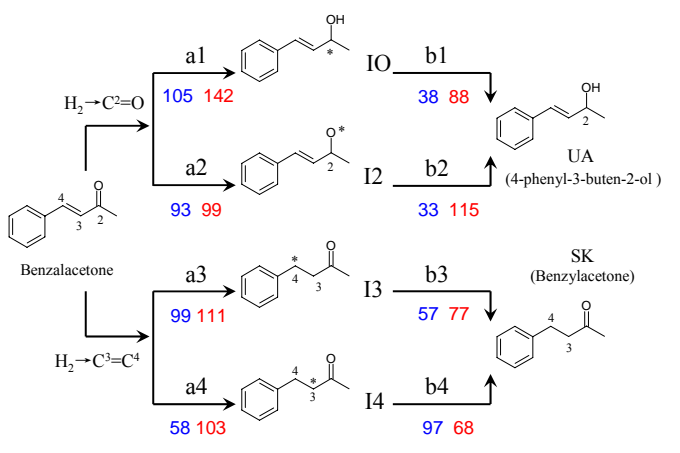

The differences in the rates of hydrogenation of benzalacetone on $\mathrm{Pt}$ and $\mathrm{Ru}$ are closely related to the binding strength of the reactant, intermediates and products. The bulky phenyl group at the $\mathrm{C}=\mathrm{C}$ bond hinder the hydrogenation of olefinic bond, but it does not alter the barriers enough to favor the formation of unsaturated alcohols.

\subsection{Hydrogenation of Cyclic Unsaturated Ketones: Ketoisophorone}

(a)

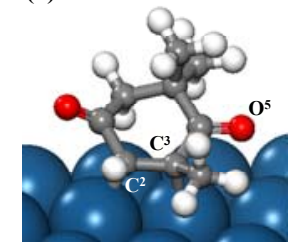

(b)

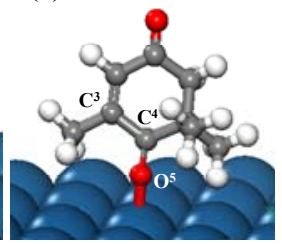

(c)

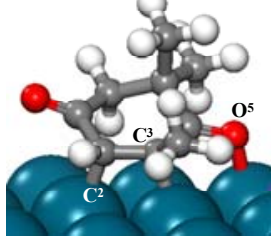

For most unsaturated ketones, the selectivity to hydrogenate the $\mathrm{C}=\mathrm{O}$ bond over the $\mathrm{C}=\mathrm{C}$ bond is rather difficult as hydrogen addition to the $\mathrm{C}=\mathrm{C}$ bond is favored over hydrogen addition to $\mathrm{C}=\mathrm{O}$ bond. Ketoisphorone, however provides methyl substituents at the carbonyl as well as at the $\mathrm{C}=\mathrm{C}$ bond that can increase the ability to add to the carbon of the carbonyl but may be limited in final formation of the saturated ketone. Interestingly experiments carried out for the selective hydrogenation of ketoisophorone show marked differences when carried out over Pd and Pt catalysts. The supported Pd catalysts tend to be more selective for the reduction of the sterically-hindered carbonyl group, leading to higher selectivity to UA as compared to Pt. The other unhindered carbonyl group is left untouched. Compared to aliphatic unsaturated ketones, the observation of UA formed during ketoisophorone hydrogenation illustrates the importance of multiple substitutions and the substituent positions. 


\section{OVERVIEW}

Altering the metal or the surface structure of a catalyst can greatly affect the interactions between the adsorbed species and the surface, and hence alter the selectivity along with the catalytic activity to unsaturated alcohols in the hydrogenation of unsaturated aldehydes and ketones. DFT calculations and kinetics analyses have shown that the low selectivity in the hydrogenation of acrolein to the unsaturated alcohol is the result of its strong binding on $\mathrm{Pt}(111)$ and over hydrogenation to form the saturated alcohol [51, 52]. The selectivity on $\operatorname{Ag}(110)$ is much better as the interaction of the unsaturated alcohol and $\mathrm{Ag}$ is much weaker thus allowing the unsaturated alcohol intermediate to readily desorb and avoid over hydrogenation [90]. In addition to the metal, the selectivity to unsaturated alcohols can also be controlled by the molecular structures of reactant. Dramatically different selectivities in the selective hydrogenation of different unsaturated ketones have been observed when carried out over the same metal catalyst [12]. Both experimental as well as theoretical studies on the influence of methyl substitution effects suggest that the aliphatic unsaturated ketones and complex unsaturated ketones with ring structures can undergo distinctive reaction dynamics even under similar steric hindrance and lead to completely different catalytic performances on the same metal catalyst $[12,13,91]$. It is therefore reasonable to expect that the interaction between reactant and metal is very sensitive to the interfacial structure that is the result of the cooperative effects between the metal and the specific structure of reactant.

The present study examines the hydrogenation of two complex substituted unsaturated ketones, benzalzcetone and ketoisophorone, on model Pt, $\mathrm{Pd}$ and $\mathrm{Ru}$ catalysts in order to examine the influence of the metal and the reactant structure on the selectivity and activity trends. In previous chapter we showed that single methyl substituents at $\mathrm{C}=\mathrm{C}$ bond could not change the rate enough to favor the $\mathrm{C}=\mathrm{O}$ over $\mathrm{C}=\mathrm{C}$ hydrogenation. In this chapter, we examine more highly-substituted substrates as well as the changes in the metal surface. Benzalacetone has a bulky phenyl group $\alpha$ to the $\mathrm{C}=\mathrm{C}$ bond which introduces a new adsorption center and should sterically hinder the hydrogenation of the olefin bond. Ketoisophorone contains three reducible groups (a sterically hindered $\mathrm{C}=\mathrm{O}$ bond, an unhindered $\mathrm{C}=\mathrm{O}$ bond and a $\mathrm{C}=\mathrm{C}$ bond) that are all present in a ring structure and show markedly different selectivities to the unsaturated alcohol from the hydrogenation of the sterically hindered $\mathrm{C}=\mathrm{O}$ bond over supported $\mathrm{Pd}$ and Pt catalysts [91]. 


\subsection{Hydrogenation of Benzalacetone over Supported Pt and Ru Catalysts}

[Adapted from: M.S. Ide, B. Hao, M. Neurock, R.H. Davis. ACS catalysis, 2 (2012) 671-683. Reproduced with permission [13], Copyright 2010, American Chemical Society.]

The hydrogenation of $\alpha, \beta$-unsaturated ketones results in the formation of unsaturated alcohols (UA) and saturated ketones (SK) which are the primary products that result from hydrogenating the $\mathrm{C}=\mathrm{O}$ bond or $\mathrm{C}=\mathrm{C}$ bonds, respectively. Both the unsaturated alcohol and the saturate ketone can be subsequently hydrogenated to form the saturated alcohol (SA). The hydrogenation of methyl vinyl ketone (MVK) as well as benzalacetone (BA) carried out experimentally over $\mathrm{Ru} / \mathrm{C}, \mathrm{Pt} / \mathrm{C}$, and $\mathrm{Pd} / \mathrm{C}$ result in the near exclusive formation of saturated ketone with conversions below 90\% (Table 3.1). Milone et al. also reported 97.5\% selectivity towards the saturated ketone at $36.3 \%$ conversion during liquid phase $\mathrm{BA}$ hydrogenation over $\mathrm{Ru} / \mathrm{Fe}_{2} \mathrm{O}_{3}$ [17]. While $\mathrm{BA}$ has a bulky phenyl group attached to the $\mathrm{C}=\mathrm{C}$ bond that can sterically hinder the hydrogenation of the $\mathrm{C}=\mathrm{C}$ bond, there was still no observed formation of the unsaturated alcohol. It was rather surprising that the benzalacetone did not show more enhanced selectivity than that of MVK. First principle density functional theory calculations were therefore carried out to understand in more detail the influence of the more bulky substituents such as the phenyl group on the hydrogenation of unsaturated ketones. More specifically we examined the selective hydrogenation paths for BA over Pt and Ru catalysts to establish the effects of metals and compare the results with those reported for MVK hydrogenation.

Table 3.1 Hydrogenation of methyl vinyl ketone (MVK) and benzalacetone over supported Pd, Pt, Ru, and Au catalysts in aqueous ${ }^{\mathrm{a}}$ and ethanol ${ }^{\mathrm{b}}$ solvent [13].

\begin{tabular}{ccccccc}
\hline \multirow{2}{*}{ Substrate } & \multirow{2}{*}{ Catalyst } & \multirow{2}{*}{$\mathrm{TOF}^{\mathrm{c}}\left(\mathrm{s}^{-1}\right)$} & Conversion (\%) & \multicolumn{3}{c}{ Selectivity (\%) } \\
& & & & $\mathrm{UA}$ & $\mathrm{SK}$ & $\mathrm{SA}$ \\
\hline \multirow{3}{*}{$\mathrm{MVK}^{\mathrm{a}}$} & $\mathrm{Pd} / \mathrm{C}$ & 2.5 & 87 & 0 & 99 & 1 \\
& $\mathrm{Pt} / \mathrm{C}$ & 0.94 & 89 & 0 & 98 & 2 \\
& $\mathrm{Ru} / \mathrm{C}$ & 0.23 & 72 & 0 & 98 & 2 \\
\hline \multirow{3}{*}{ Benzalacetone $^{\mathrm{b}}{ }^{\mathrm{s}}$} & $\mathrm{Pd} / \mathrm{C}$ & 9.4 & 69 & 0 & 96 & 4 \\
& $\mathrm{Pt} / \mathrm{C}$ & 1.9 & 69 & 0 & 95 & 5 \\
& $\mathrm{Ru} / \mathrm{C}$ & 1.2 & 64 & 0 & 95 & 5 \\
\hline
\end{tabular}

Reaction conditions: $0.2 \mathrm{M}$ substrate, $\mathrm{S}: \mathrm{M}_{\text {surf }} \sim 5000, \mathrm{pH}_{2}=2 \mathrm{~atm}, \mathrm{~T}=333 \mathrm{~K} .{ }^{\mathrm{c}}$ Turnover frequency is reported at $\sim 20 \%$ conversion. ${ }^{\mathrm{d}}$ Selectivity is reported at the level of conversion in the table.

\section{Computational Details}

The bulk lattice constants for Pt and $\mathrm{Ru}$ involved in the hydrogenation of benzalacetone were initially set to the experimental values of $\mathrm{a}=3.92 \AA$ for $\mathrm{Pt}$ and $\mathrm{a}=2.71 \AA$ for $\mathrm{Ru}[92,93]$ and then optimized within VASP. The optimized values of $\mathrm{a}=3.99 \AA$ and $2.70 \AA$ were subsequently used in the construction of the $\operatorname{Pt}(111)$ and 
$\mathrm{Ru}(0001)$ surfaces. The $\mathrm{Pt}(111)$ and $\mathrm{Ru}(0001)$ surfaces were modeled using a $4 \times 4$ unit cell to accommodate the reacting species and minimize spurious cell to cell interactions, giving a constant surface coverage of $1 / 16$ species ML (1 adsorbate per unit cell) which is much lower than the saturation coverage to avoid strong repulsive interaction between adsorbates. Higher surface coverage of the unsaturated ketone and hydrogen reactants will result in lateral repulsive interactions, which decrease the calculated activation barriers for hydrogenation reactions [65]. The adsorption energies were calculated as $\Delta E_{\text {ads }}=E_{\text {metal }+ \text { adsorbate }}-E_{\text {adsorbate }}-$ $E_{\text {metal }}$, where a negative value indicates that adsorption is an exothermic process. While the experimentallymeasured hydrogenation rates were evaluated in the presence of a solvent, for the sake of simplicity, all of the DFT calculated reactions reported herein were carried out in vapor phase. Our previous results that were carried out in aqueous media over metal surfaces indicate that barriers for hydrogenating oxygenates in the presence of a polar solvent can decrease by as much as $20 \mathrm{~kJ} \mathrm{~mol}^{-1}$ compared to those determined without a solvent due to the stabilization of the partially-charged transition states by the polar solvent $[31,94]$.

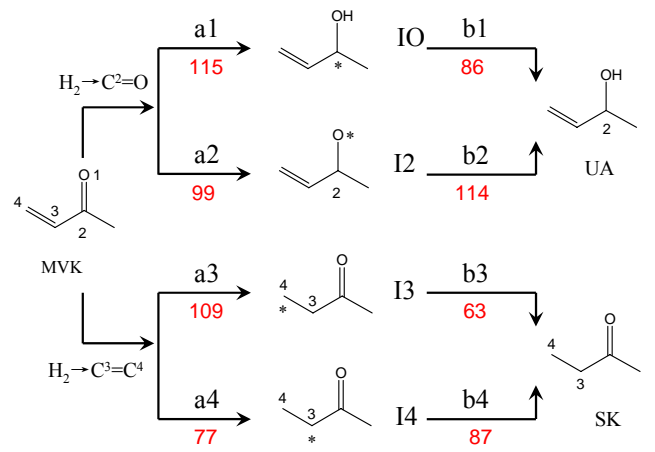

(a)

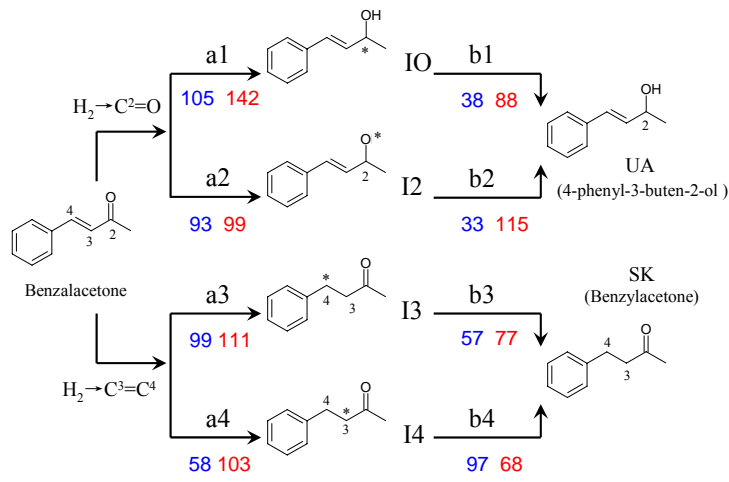

(b)

Figure 3.1 Competitive hydrogenation paths for (a) MVK and (b) benzalacetone. The first hydrogenation intermediates IO, I2, I3 and I4 are named after the hydrogen addition-centers. Pathways a1-a4 indicate the elementary reactions of the first hydrogenation step, and b1-b4 refer to the second hydrogenation reaction. The values highlighted in blue refer to activation barriers $\left(\mathrm{kJ} \mathrm{mol}^{-1}\right)$ occurring on $\operatorname{Pt}(111)$ and while those in red refer to the values calculated on $\operatorname{Ru}(0001)$. '*' here refers to an unsaturated carbon or oxygen that remains bonded to the metal surface after the initial hydrogenation step.

\section{Hydrogenation of Benzalacetone}

The calculations examined the hydrogenation of BA over both the model $\mathrm{Pt}(111)$ and $\mathrm{Ru}(0001)$ surfaces. Benzalacetone was found to adsorb on the $\mathrm{Pt}(111)$ surface either via its aromatic ring and $\mathrm{C}=\mathrm{C}$ bond (Figure 3.2a) or only via its $\mathrm{C}=\mathrm{O}$ bond (Figure $3.2 \mathrm{~b}$ ). The former configuration has a significantly stronger adsorption energy $\left(-100 \mathrm{~kJ} \mathrm{~mol}^{-1}\right)$ than the latter one $\left(-17 \mathrm{~kJ} \mathrm{~mol}^{-1}\right.$, Table 3.2) as it involves the adsorption of both the aromatic ring $\left(\sim-70 \mathrm{~kJ} \mathrm{~mol}^{-1}\right)$ as well as the unsaturated $\mathrm{C}=\mathrm{C}$ bond. Similar to $\mathrm{BA}$, unsaturated aldehydes such as acrolein also interact with $\mathrm{Pt}(111)$, mainly via their $\mathrm{C}=\mathrm{C}$ bond, but only a weak interaction with the oxygen 
atom was observed [41]. On $\mathrm{Pt}(111)$, we consider the hydrogenation of the $\mathrm{C}=\mathrm{C}$ bond starting from the adsorbed state of Figure 3.2a and the hydrogenation of the $\mathrm{C}=\mathrm{O}$ bond starting from adsorption state of Figure $3.2 b$.

The binding energies for the reactants as well as the intermediates (IO-I4, as shown in Figure 3.2b) of BA were found to be much stronger on Ru surface than on Pt surface, as indicated by Table 3.2. The adsorption of BA, for example, was calculated to be $-272 \mathrm{~kJ} \mathrm{~mol}^{-1}$ on $\mathrm{Ru}(0001)$, which is $-172 \mathrm{~kJ} \mathrm{~mol}^{-1}$ stronger than that on $\mathrm{Pt}(111)$. The stronger adsorption on $\mathrm{Ru}$ over $\mathrm{Pt}$ is attributed to the stronger $\mathrm{Ru}-\mathrm{C}$ and $\mathrm{Ru}-\mathrm{O}$ bonds than the Pt$\mathrm{C}$ and Pt-O bonds as well as the more favorable orientation of the phenyl, $\mathrm{C}=\mathrm{C}$, and $\mathrm{C}=\mathrm{O}$ bonds on the $\mathrm{Ru}(0001)$ surface (Figure 3.2c) as compared to $\mathrm{Pt}(111)$. Similarly, the intermediates and products were also bound much more strongly to $\mathrm{Ru}$ than to Pt, for example, IO $\left(-374 \mathrm{~kJ} \mathrm{~mol}^{-1}\right.$ on Ru versus $\left.-156 \mathrm{~kJ} \mathrm{~mol}^{-1} \mathrm{on} \mathrm{Pt}\right)$ and UA $\left(-228 \mathrm{~kJ} \mathrm{~mol}^{-1}\right.$ on Ru versus $-27 \mathrm{~kJ} \mathrm{~mol}^{-1}$ on Pt). Both of the primary products UA and SK were bound to surface (Figure 3.6), which indicates a possibility of being further hydrogenated to saturated alcohol. Analogous to the case with MVK, desorption of the saturated ketone of benzalacetone was calculated to require much lower energy when compared to the unsaturated alcohol over the $\mathrm{Ru}(0001)$ surface.
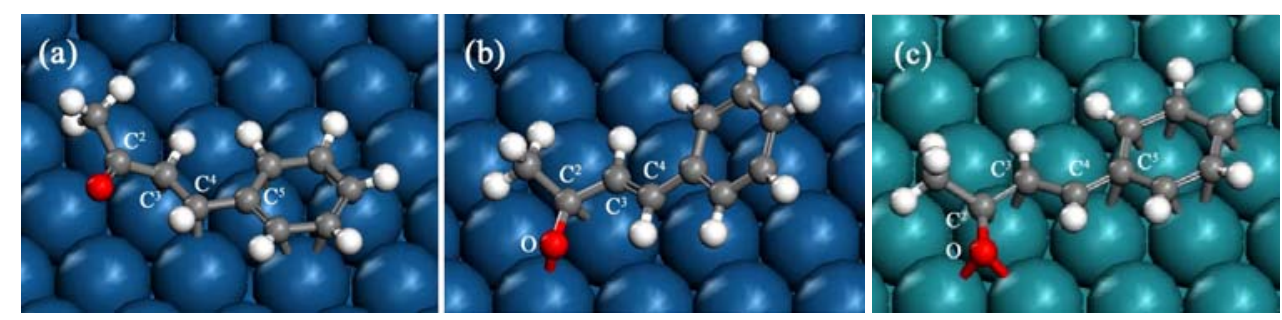

Figure 3.2 Adsorption configurations for benzalacetone (a) over $\mathrm{Pt}(111)$ via the aromatic ring and $\mathrm{C}=\mathrm{C}$ bond; (b) over $\mathrm{Pt}(111)$, only via the $\mathrm{C}=\mathrm{O}$ bond; (c) over $\mathrm{Ru}(0001)$ surface.

Table 3.2 Adsorption energies $\left(\mathrm{kJ} \mathrm{mol}^{-1}\right)$ for the reactants (indicated by UK), intermediates and products of benzalacetone hydrogenation on $\mathrm{Pt}(111)$ and $\mathrm{Ru}(0001)$ and MVK hydrogenation on $\mathrm{Ru}(0001)$.

\begin{tabular}{cccc}
\hline Species & $\begin{array}{c}\text { Benzalacetone } \\
\mathrm{Pt}(111)\end{array}$ & $\begin{array}{c}\text { Benzalacetone } \\
\mathrm{Ru}(0001)\end{array}$ & $\begin{array}{c}\text { MVK } \\
\mathrm{Ru}(0001)\end{array}$ \\
\hline UK & $-100^{\mathrm{a}},-17^{\mathrm{b}}$ & -272 & -148 \\
$\mathrm{IO}$ & -156 & -374 & -177 \\
$\mathrm{I} 2$ & -144 & -335 & -300 \\
$\mathrm{I} 3$ & -156 & -297 & -195 \\
$\mathrm{I} 4$ & -175 & -305 & -178 \\
UA & -27 & -228 & -113 \\
SK & -55 & -122 & -28 \\
\hline${ }^{\mathrm{a}}$ Adsorbed through aromatic ring and $\mathrm{C}=\mathrm{C}{ }^{\mathrm{b}}$ Adsorbed through $\mathrm{C}=\mathrm{O}$.
\end{tabular}


Figure 3.1 depicts the possible competitive paths for the hydrogenation of benzalacetone over $\mathrm{Pt}$ and $\mathrm{Ru}$ surfaces. The calculated activation barriers over Pt(111) are depicted in values shown in blue whereas the values on $\mathrm{Ru}(0001)$ are shown in red. If the activation energies for the addition of the first hydrogen are calculated with respect to adsorbed BA and atomic hydrogen, they follow very similar trends on both Pt and $\mathrm{Ru}:$

over Pt(111): $\quad \mathrm{C}^{4}$-(phenyl) path a4 $<\mathrm{C}^{2}-(\mathrm{O})$ path a2 $<\mathrm{C}^{3}-\left(\mathrm{CH}_{2}\right)$ path a3 $<\mathrm{O}^{1}$ path a1 over $\mathrm{Ru}(0001)$ : $\quad \mathrm{C}^{2}-(\mathrm{O})$ path a2 $<\mathrm{C}^{4}$-(phenyl) path a4 $<\mathrm{C}^{3}-\left(\mathrm{CH}_{2}\right)$ path a3 $<\mathrm{O}^{1}$ path a1

In comparison with the results for MVK over Ru surface $\left(\mathrm{C}^{4}\right.$ path $\mathrm{a} 4<\mathrm{C}^{2}-(\mathrm{O})$ path $\mathrm{a} 2<\mathrm{C}^{3}$ path $\mathrm{a} 3<\mathrm{O}^{1}$ path a1), the only difference of BA over Ru surface lies in the order of the hydrogen addition steps for $\mathrm{C}^{2}-(\mathrm{O})$ and $\mathrm{C}^{4}$-(phenyl), which is due to the introduction of the phenyl group at the $\mathrm{C}^{4}$ position.
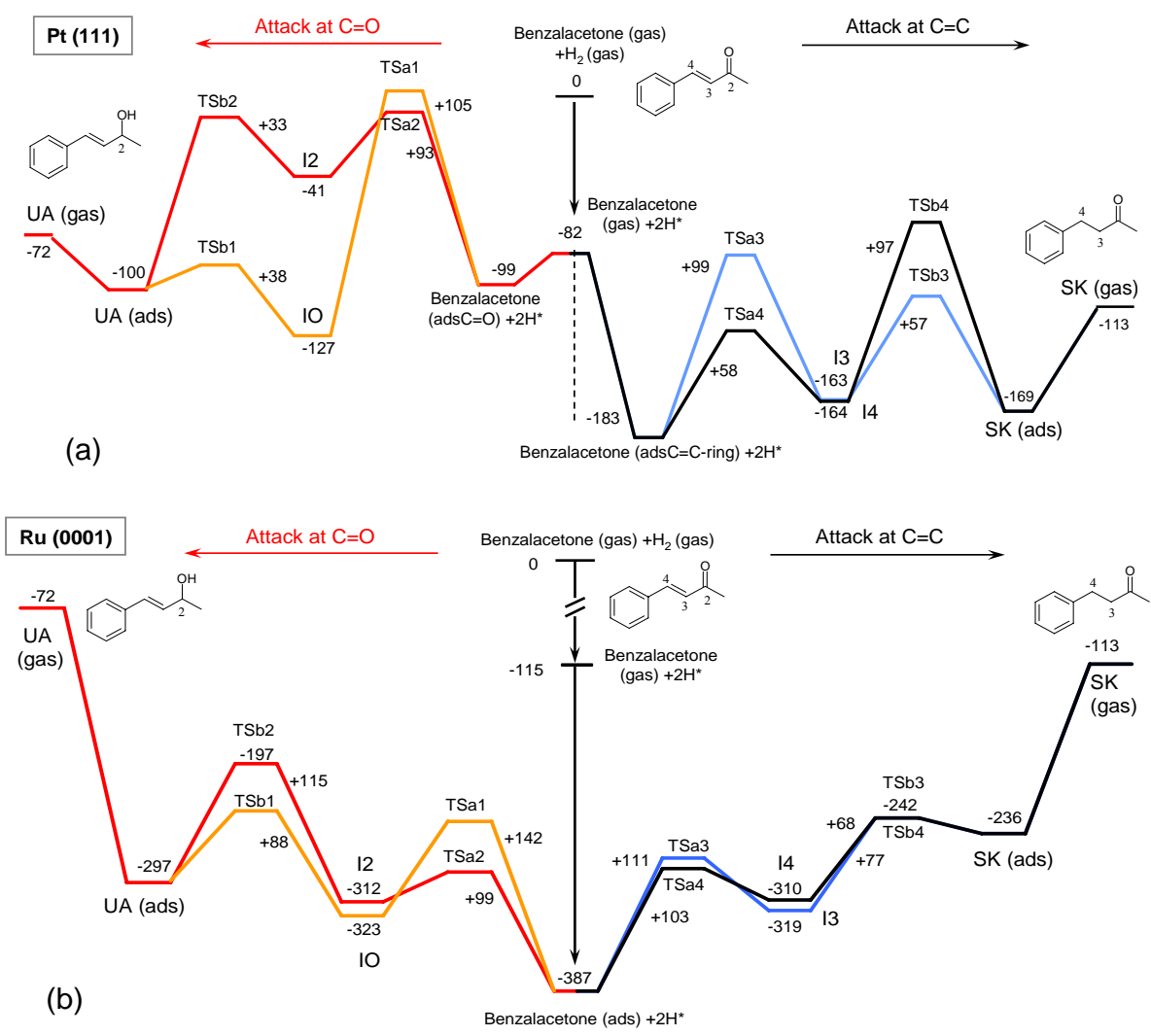

Figure 3.3 Potential energy profiles of the benzalacetone hydrogenation on (a) $\operatorname{Pt}(111)$ and (b) $\operatorname{Ru}(0001)$. Reaction energies and activation barriers are in $\mathrm{kJ} \mathrm{mol}^{-1}$.

On Pt, the observed selectivity to the UA is controlled by the significantly higher activation barriers to hydrogenate $\mathrm{C}=\mathrm{O}$ bond than those to hydrogenate the $\mathrm{C}=\mathrm{C}$ bond (Figure 3.3a). The optimal energy path for 
hydrogenating BA over Pt(111) proceeds by the addition of hydrogen to the carbon that is $\alpha$ to the aromatic ring via path a4 which has an intrinsic barrier of only $58 \mathrm{~kJ} \mathrm{~mol}^{-1}$. This path is significantly more favorable than the hydrogenation of the carbonyl bond, which shows high barriers for the initial addition of hydrogen to oxygen $\left(105 \mathrm{~kJ} \mathrm{~mol}^{-1}\right)$ as well as to the carbon $\left(93 \mathrm{~kJ} \mathrm{~mol}^{-1}\right)$. The subsequent hydrogenation of the resulting I2 and IO intermediates were calculated to be only 33 and $38 \mathrm{~kJ} \mathrm{~mol}^{-1}$. The transition state structures for the favorable path, a4-b4, are shown in Figure3.4. If we take into account the very weak adsorption of the carbonyl to the $\mathrm{Pt}(111)$ surface, the hydrogenation of $\mathrm{C}=\mathrm{O}$ is clearly not favored and $\mathrm{UA}$ is predicted to be a very minor product, which is consistent with the experimental results.

The calculated activation barriers over $\mathrm{Ru}(0001)$, shown in red in Figure $3.1 \mathrm{~b}$ and Figure $3.3 \mathrm{~b}$, were found to be significantly higher than those over Pt(111) for most of the hydrogen addition steps, which is consistent with the stronger heat of adsorption of BA on Ru than on Pt as well as the much lower experimental turnover frequencies over $\mathrm{Ru}$ than $\mathrm{Pt}$ (Table 3.1). This is due to the strong interaction of the phenyl group in BA to $\mathrm{Ru}(0001)$ than on $\operatorname{Pt}(111)$, which leads to an increase in the steric repulsion at the $\mathrm{C}^{4}$ site thus making it more difficult to add hydrogen to the $\mathrm{C}^{4}$ position. The barrier for this initial hydrogen addition step was calculated to be significantly higher on $\mathrm{Ru}\left(103 \mathrm{~kJ} \mathrm{~mol}^{-1}\right)$ than that on $\mathrm{Pt}\left(58 \mathrm{~kJ} \mathrm{~mol}^{-1}\right)$.

A closer analysis of $\mathrm{C}=\mathrm{O}$ and $\mathrm{C}=\mathrm{C}$ hydrogenation over $\mathrm{Ru}(0001)$ suggests that the initial hydrogen addition to the carbon of $\mathrm{C}=\mathrm{O}$ via path a2 $\left(99 \mathrm{~kJ} \mathrm{~mol}^{-1}\right)$ is more favorable than the addition of hydrogen to the $\mathrm{C}^{4}$ of the $\mathrm{C}=\mathrm{C}$ bond via path a4 $\left(103 \mathrm{~kJ} \mathrm{~mol}^{-1}\right)$. This can be attributed to the enhanced stabilization of the transition state in forming a strong Ru-O bond of the alkoxide intermediate over the weaker Ru-CHR bond in forming the alkyl intermediate. The difference is also reflected in the stronger binding of the alkoxide intermediate $(-335 \mathrm{~kJ}$ $\mathrm{mol}^{-1}$ for I2) over that of the alkyl intermediate $\left(-305 \mathrm{~kJ} \mathrm{~mol}^{-1}\right.$ for I4) that forms. The activation barrier for the subsequent hydrogenation of the I2 alkoxy intermediate via path b2 however is considerably higher (115 kJ $\left.\mathrm{mol}^{-1}\right)$ than that for the subsequent hydrogenation of the $\mathrm{I} 4$ alkyl intermediate via path b4 $\left(68 \mathrm{~kJ} \mathrm{~mol}^{-1}\right)$.

A comparison of the potential energy surfaces for both paths depicted in Figure 3.3b indicates that the I2 and I4 intermediates are likely quasi-equilibrated and as such the apparent barriers can be taken from the adsorbed initial state and the transition state energies TSb2 (energy level -197 kJ mol ${ }^{-1}$ ) and TSb4 (energy level -242 kJ $\mathrm{mol}^{-1}$ ), which would result in an overall barrier of $190 \mathrm{~kJ} \mathrm{~mol}^{-1}$ (from the adsorbed reactant states) for route a2b2 and $145 \mathrm{~kJ} \mathrm{~mol}^{-1}$ for route a4-b4, respectively. Alternatively, one can also reference the energies of TSb2 and TSb4 from the gas phase to show that similar trends in selectivity will be found, but with lower effective barriers. The results indicate that while the phenyl ring increases the barrier to hydrogenate the $\mathrm{C}=\mathrm{C}$ bond in $\mathrm{BA}, \mathrm{C}=\mathrm{C}$ bond hydrogenation is still much more favorable than $\mathrm{C}=\mathrm{O}$ hydrogenation and the saturated ketone is still the major product that would be formed over supported $\mathrm{Ru}$, which is consistent with the experimental results reported above. 
Although the apparent barrier for the selective hydrogenation of BA to form the saturated ketone via the path a4-b4 is lower than that to form the unsaturated ketone via the path a1-b1, the difference is only $8 \mathrm{~kJ} \mathrm{~mol}^{-1}$, which would suggest that some unsaturated alcohol might also be formed. The unsaturated alcohol, however, is strongly bound to the Ru surface and based on the calculated barrier it should readily hydrogenate to the saturated alcohol before desorbing, which is consistent with the experimental results reported in Table 3.1 indicating that unsaturated alcohols are not observed over the various metals during BA hydrogenation.
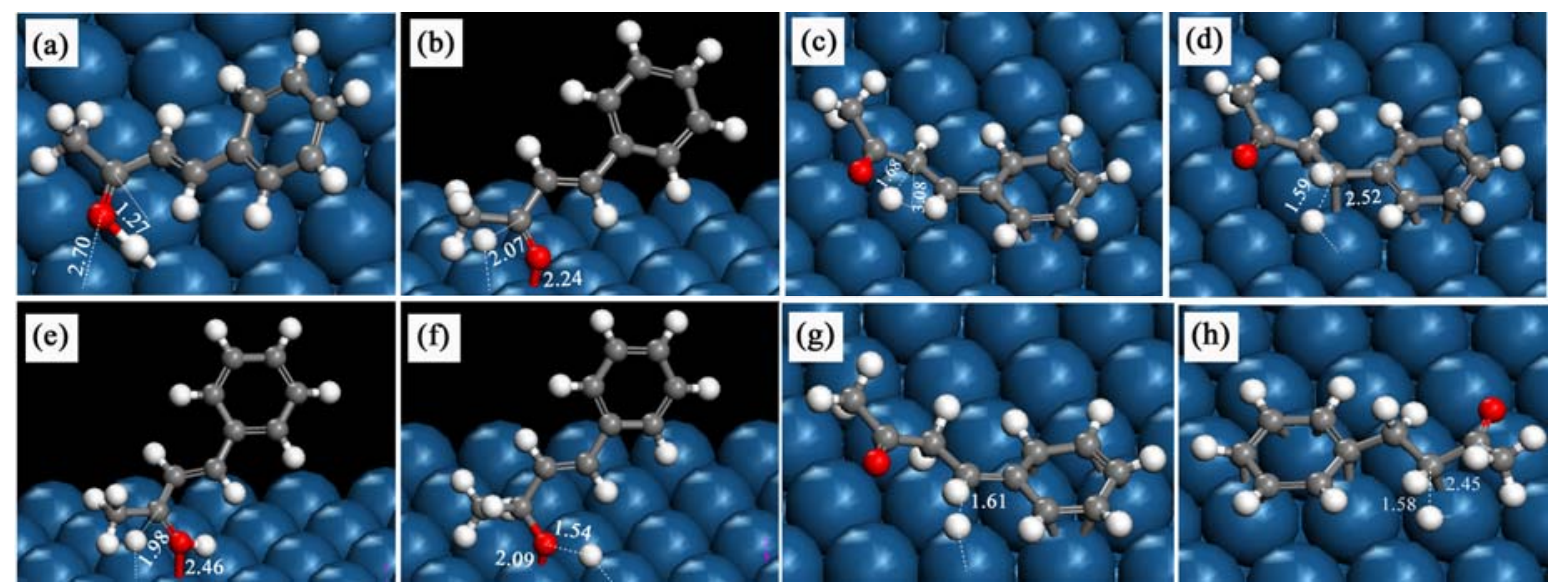

Figure 3.4 Transition states configurations for (a) path a1; (b) path a2; (c) path a3; (d) path a4; (e) path b1; (f) path $\mathrm{b} 2 ;$ (g) path b3; (h) path b4 for benzalacetone hydrogenation over Pt (111). Bond lengths are in $\AA$.
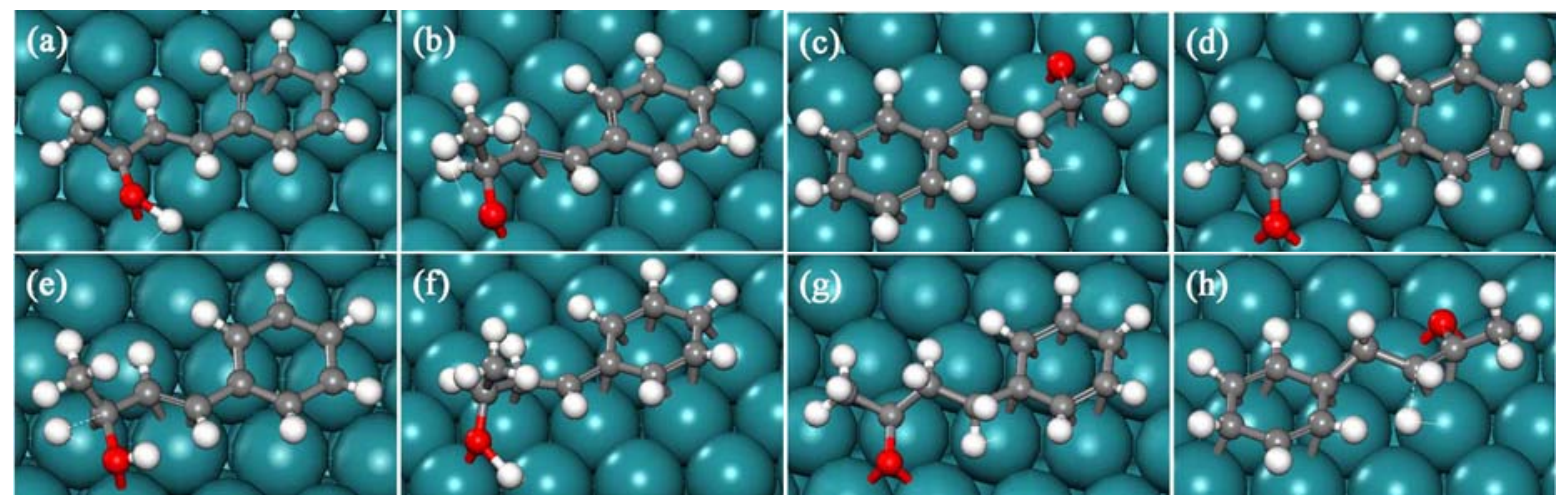

Figure 3.5 Transition states configurations of (a) path a1; (b) path a2; (c) path a3; (d) path a4; (e) path b1; (f) path b2; (g) path b3; (h) path b4 for benzalacetone hydrogenation over $\mathrm{Ru}(0001)$. 

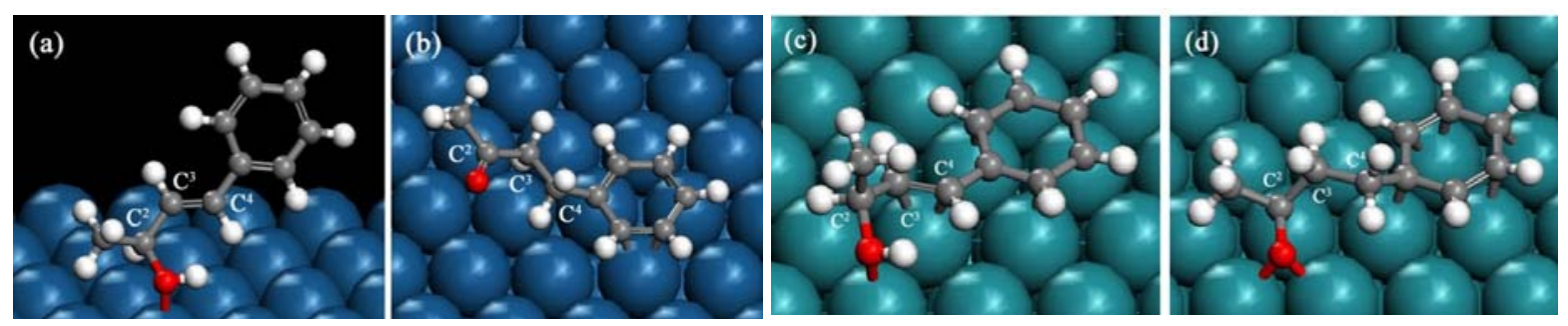

Figure 3.6 Adsorption configurations for the products of benzalacetone hydrogenation (a) UA on Pt(111); (b) SK on $\mathrm{Pt}(111)$; (c) UA on $\mathrm{Ru}(0001)$; (d) SK on $\mathrm{Ru}(0001)$.

Although a direct quantitative comparison of MVK and BA hydrogenation is not possible because of the different solvents used in each experiment, we can compare the results from theory carried out in the absence of solvent to understand qualitatively the differences between MVK and BA. As depicted in Figure 3.1, the substitution of the methyl group with a phenyl group $\alpha$ to the $\mathrm{C}^{4}$ center increased the barrier to add the first hydrogen to the $\mathrm{C}=\mathrm{C}$ bond by $26 \mathrm{~kJ} \mathrm{~mol}^{-1}$, since the barrier for the a4 path of MVK is only $77 \mathrm{~kJ} \mathrm{~mol}^{-1}$ whereas that for BA is $103 \mathrm{~kJ} \mathrm{~mol}^{-1}$. The strongly bound phenyl substituent sterically hinders the approach of the bound hydrogen in the transition state and significantly increases the adsorption strength of BA over MVK, both of which result in a higher $\mathrm{C}=\mathrm{C}$ activation energy for $\mathrm{BA}$ over $\mathrm{MVK}$.

While the phenyl substitution increase the intrinsic barrier for hydrogenating the $\mathrm{C}=\mathrm{C}$ bond of $\mathrm{BA}$ as compared to $\mathrm{MVK}$, it does not increase the barriers high enough to favor the carbonyl hydrogenation. The apparent barrier for $\mathrm{C}=\mathrm{C}$ hydrogenation is still lower than that for the $\mathrm{C}=\mathrm{O}$ bond as indicated by Figure 3.3b. In the hydrogenation of unsaturated aldehyde such as cinnamaldehyde [13] the substitution of phenyl group has successfully decreased the relative hydrogenation rate of the olefinic bond and higher selectivity to UA was observed as compared to either crotonaldehyde or 3-methylcrotonaldehyde [26]. However, the presence of the bulky phenyl group in the unsaturated ketone does not help in converting the carbonyl. The experimental TOF results have indicated that the hydrogenation of $\mathrm{C}=\mathrm{C}$ bond in the unsaturated ketone was much faster than in unsaturated aldehyde over supported $\mathrm{Pd}, \mathrm{Pt}$ and $\mathrm{Ru}$ catalysts [13]. The theoretical results presented herein suggested that while the phenyl substitution to the olefinic $\mathrm{C}=\mathrm{C}$ bond of unsaturated ketone can lower the $\mathrm{C}=\mathrm{C}$ reaction rate, it cannot change the rate enough to alter the selectivity trend since the hydrogenation of $\mathrm{C}=\mathrm{C}$ is still easier and faster than that of carbonyl. The major product is still the saturated ketone which is consistent with experimental results reported over $\mathrm{Ru} / \mathrm{C}$ catalyst [13]. 


\section{Conclusions}

The experimental rate and selectivity for hydrogenation of methyl vinyl ketone (MVK) and benzalacetone (BA) over supported $\mathrm{Pt} / \mathrm{C}$ and $\mathrm{Ru} / \mathrm{C}$ were compared under similar conditions. Theoretical calculations were carried out to explore the hydrogenation mechanisms and energetics of benzalacetone (BA) over Pt and Ru catalysts, to establish the effect of metal, and draw comparisons with MVK, and to investigate the influence of steric inhibition and electronic effects of bulky phenyl at the $\mathrm{C}=\mathrm{C}$ bond on the selectivity. The binding energies for the reactants as well as the intermediates of BA were found to be much stronger on Ru surface than on Pt. Both primary products UA and SK were strongly bound to surface, which indicates that these intermediates may be further hydrogenated to saturated alcohol. However, desorption of the SK of benzalacetone was calculated to require much lower energy when compared to the UA over the Ru surface.

The calculated barriers for benzalacetone hydrogenation were found to be significantly higher over $\mathrm{Ru}$ as compared to Pt, which is consistent with the lower experimental TOF reported over Ru than Pt. Over Pt, the barriers for the addition of the first hydrogen to the $\mathrm{C}=\mathrm{O}$ bond are considerably higher than that to hydrogenate the $\mathrm{C}=\mathrm{C}$ bond. Over $\mathrm{Ru}$ surface, while the presence of phenyl substituent of the $\mathrm{C}=\mathrm{C}$ bond in benzalacetone increases the $\mathrm{C}=\mathrm{C}$ hydrogenation barrier by $26 \mathrm{~kJ} \mathrm{~mol}^{-1}$ when compared to the methyl substituted $\mathrm{C}=\mathrm{C}$ bond in $\mathrm{MVK}$, it does not change the relative $\mathrm{C}=\mathrm{C}$ to $\mathrm{C}=\mathrm{O}$ hydrogenation barriers enough to affect the selectivity trend. This is consistent with experimental results reported over Ru that showed the substitution of the phenyl group $\alpha$ to the $\mathrm{C}=\mathrm{C}$ bond slows down the rate of $\mathrm{C}=\mathrm{C}$ hydrogenation over all metals investigated.

The theoretical results here show that the differences in the rates of hydrogenation of BA on Pt and $\mathrm{Ru}$ are closely related to the binding strength of the reactant, intermediates and products. While the bulky phenyl group $\alpha$ to the $\mathrm{C}=\mathrm{C}$ bond hinders the addition of hydrogen to the olefinic bond compared to that of carbonyl, it does not alter the barriers enough to favor the formation of unsaturated alcohols. Single substituent (such as methyl, aromatic ring) at $\mathrm{C}=\mathrm{C}$ bond did not change the rate of $\mathrm{C}=\mathrm{C}$ hydrogenation enough to favor the reaction of $\mathrm{C}=\mathrm{O}$ bond of unsaturated ketone. The multiple substitutions at the hydrocarbon backbones of more highlysubstituted substrates may begin to alter the selectivity. 


\subsection{Hydrogenation of Cyclic Unsaturated Ketones: Ketoisophorone}

In the previous unsaturated ketone molecules that we examined which include MVK and benzalacetone, we showed that while single substituents (methyl group, aromatic ring) at the $\mathrm{C}=\mathrm{C}$ increased the barriers for hydrogen addition to the $\mathrm{C}=\mathrm{C}$ bond and lowered the barriers for hydrogen addition to the carbon of the $\mathrm{C}=\mathrm{O}$ bond, they could not alter the selectivity as the strongly bound alkoxide that forms on $\mathrm{Ru}$ and Pt could not be readily hydrogenated to form the unsaturated alcohol. We find here that the addition of a second substituent of the hydrocarbon backbone can begin to alter the selectivity so as to enhance the rate of $\mathrm{C}=\mathrm{O}$ hydrogenation over $\mathrm{C}=\mathrm{C}$ hydrogenation.

Ketoisophorone (KIP) is a highly functionalized unsaturated ketone that is comprised of three reducible $\mathrm{C}=\mathrm{O}$ and $\mathrm{C}=\mathrm{C}$ groups. The methyl substituent at the $\mathrm{C}^{3}$ position along with the $\mathrm{C}^{1}=\mathrm{O}$ group attached to the $\mathrm{C}^{2}$ position can act to limit $\mathrm{H}$ access to both the $\mathrm{C}^{2}$ and $\mathrm{C}^{3}$ carbons of $\mathrm{C}=\mathrm{C}$ bond. The different sites on KIP ultimately lead to a range of different hydrogenation products that can be formed as can be seen in Figure 3.7. The hydrogenation of KIP at the $\mathrm{C}=\mathrm{C}$ double bond produces levodione ((6R)-2,2,6-trimethylcyclohexane-1,4dione), a key building block in the synthesis of 3-hydroxy-carotinoids, which are a range of pigments and flavorings and also precursors of vitamin A $[95,96]$. The unsaturated alcohol, 4-hydroxyisophorone, produced by the selective hydrogenation of the $\mathrm{C}=\mathrm{O}$ bond, is an important constituent of saffron, which is an intermediate in the synthesis of valuable pharmaceuticals, natural pigments, flavorings and biologically active products $[97,98]$. Biocatalysts such as baker's yeast can result in selectivities of $83 \%$ to levodione and only $6 \%$ to 4 -hydroxyisophorone [91]. The rate of reaction, however is rather slow and there is a relative intolerance to changes in the reaction conditions [60].

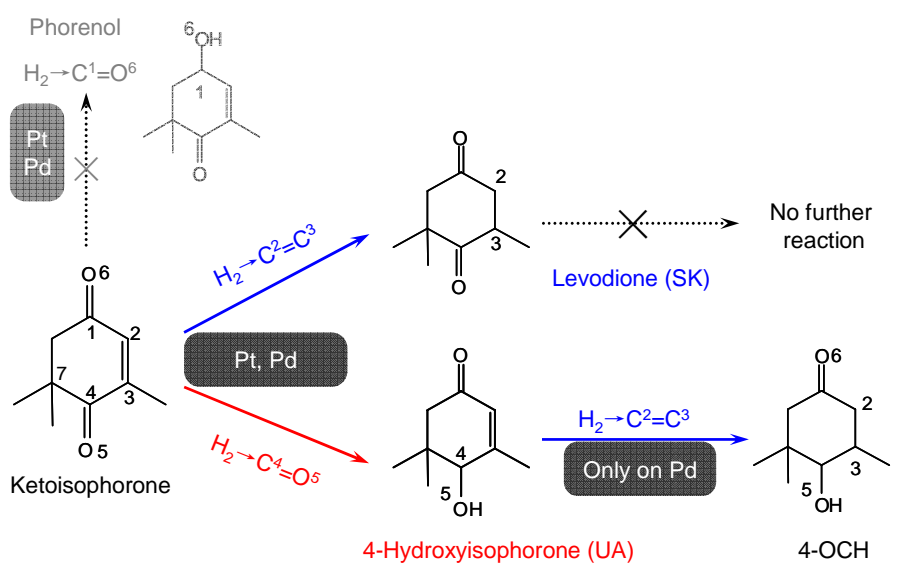

Figure 3.7 Competitive pathways and possible products in the catalytic hydrogenation of ketoisophorone (KIP) over supported Pt and Pd catalysts. 
The selectivity to form the desired unsaturated alcohol over the saturated ketone differs significantly over supported Pd and Pt catalysts for ketoisophorone hydrogenations [54, 91]. The hydrogenation of KIP over $\mathrm{Pt} / \mathrm{Al}_{2} \mathrm{O}_{3}$ results in 1:1 mixture of the saturated ketone levodione (SK) and the allylic alcohol 4hydroxyisophorone (UA). The hydrogenation of KIP over $\mathrm{Pd} / \mathrm{Al}_{2} \mathrm{O}_{3}$, however, results in selectivity of greater than $90 \%$ to 4-hydroxyisophorone (unsaturated alcohol) over levodione (saturated ketone) [91]. If the reaction is continued out to $\sim 100 \%$ conversion over $\mathrm{Pd} / \mathrm{Al}_{2} \mathrm{O}_{3}$, the 4-hydroxyisophorone (UA) is further hydrogenated to form the hydroxy-ketone 4-OCH, while the amount of levodione remains constant. The subsequent conversion of unsaturated alcohol to the saturated alcohol does not occur, however, on $\mathrm{Pt} / \mathrm{Al}_{2} \mathrm{O}_{3}$ [91]. In order to understand the different reactivity and selectivities trends for KIP hydrogenation over Pt and Pd, we carried out first principle theoretical calculations over model $\mathrm{Pt}(111)$ and $\mathrm{Pd}(111)$ surfaces. We specifically examine the influence of metal on the adsorption and reactivity and compare the mechanisms that control the hydrogenation of the $\mathrm{C}=\mathrm{C}$, sterically-hindered carbonyl $\mathrm{C}^{4}=\mathrm{O}^{5}$ and non-hindered $\mathrm{C}^{1}=\mathrm{O}^{6}$ bond of KIP.

\section{Computational Details}

Periodic density functional theory calculations were carried out using the Vienna ab-initio simulation program (VASP) [68] with the Perdew-Wang 91 form of GGA along with ultrasoft pseudopotentials (USPP) [69, 70]. The bulk lattice constants for Pt and Pd were initially set to the experimental values and then optimized within VASP, resultin in optimized values of $\mathrm{a}=3.99 \AA$ for Pt and $3.89 \AA$ for Pd were subsequently used in the construction of the $\operatorname{Pt}(111)$ and $\operatorname{Pd}(111)$ surfaces. The $\operatorname{Pd}(111)$ and $\operatorname{Pt}(111)$ surfaces were modeled using a $4 \times 4$ unit cell to accommodate the reacting species and minimize spurious cell to cell interactions, giving a constant surface coverage of 1/16 species ML (1 adsorbate per unit cell) which is much lower than the saturation coverage to avoid strong repulsive interaction between adsorbates. The structures for all of the reactant, intermediates and products were optimized until the force on each atom was less than $0.05 \mathrm{eV} / \AA$. A three step approach was used to determine the lowest energy reactant, intermediate and product structures. The first and second steps use a $3 \times 3 \times 1$ Monkhorst-Pack k-point mesh [71] to sample the Brillouin zone [72] for both the first and second steps and a $6 \times 6 \times 1 \mathrm{k}$-point mesh was used for the third step. In the third and final step, we carry out a static single-point calculation. Adsorption energies are calculated as $\Delta E_{a d s}=E_{\text {metal }+ \text { adsorbate }}$ $E_{\text {adsorbate }}-E_{\text {metal }}$, where a negative value indicates that the adsorption is exothermic. The nudged elastic band (NEB) method [74-77] was combined with the dimer method [78-80] to isolate the specific transition state. In NEB calculation an initial series of intermediate images were refined using a $3 \times 3 \times 1$ k-point until the maximum force on each atom decreased to $0.20 \mathrm{eV} / \AA$. The dimer calculations were carried out by $3 \times 3 \times 1 \mathrm{k}$ point until the maximum atomic forces converged to $0.05 \mathrm{eV} / \AA$, and finally a $6 \times 6 \times 1 \mathrm{k}$-point was used to determine the energy of the transition state (TS) in a static single-point calculation.

\section{Results and Discussions}


The complexity of the ketoisopherone structure makes it difficult to discern its modes of adsorption as it can bind in a number of different configurations. On Pt(111), KIP can adsorb via the $\mathrm{C}^{2}=\mathrm{C}^{3}$ bond (Figure 3.8a) or via the oxygen atom of the sterically-hindered carbonyl, $\mathrm{C}^{4}=\mathrm{O}^{5}$ (Figure 3.8b). Both configurations lead to weak interactions with the Pt surface. The former $\left(-18 \mathrm{~kJ} \mathrm{~mol}^{-1}\right)$ is slightly more favorable over the latter $\left(-15 \mathrm{~kJ} \mathrm{~mol}^{-1}\right.$, Table 3.3). They are considered as the favorable configurations for hydrogenating the $\mathrm{C}^{2}=\mathrm{C}^{3}$ bond and carbonyl $\mathrm{C}^{4}=\mathrm{O}^{5}$ groups, respectively. On $\mathrm{Pd}(111)$, KIP binds much more strongly $\left(-42 \mathrm{~kJ} \mathrm{~mol}^{-1}\right)$ than it does on $\operatorname{Pt}(111)$ and it adopts an $\eta^{3}$ orientation that allows both the $\mathrm{C}^{2}=\mathrm{C}^{3}$ bond and the oxygen $\mathrm{O}^{5}$ to interact with the surface simultaneously (Figure 3.8c). Previous theoretical work suggested that the difference in the adsorption strengths of different molecules on Pd vs. Pt is related to the larger $d$ band widths on Pt and Ir over that on $\mathrm{Pd}(\mathrm{Pd}<\mathrm{Pt}<\mathrm{Ir} \approx \mathrm{Os})$, which result in more enhanced repulse between the metal and the $\mathrm{C}=\mathrm{C}$ bond of the adsorbate and thus weakening the chemisorption via the $\mathrm{C}=\mathrm{C}$ bond [99].
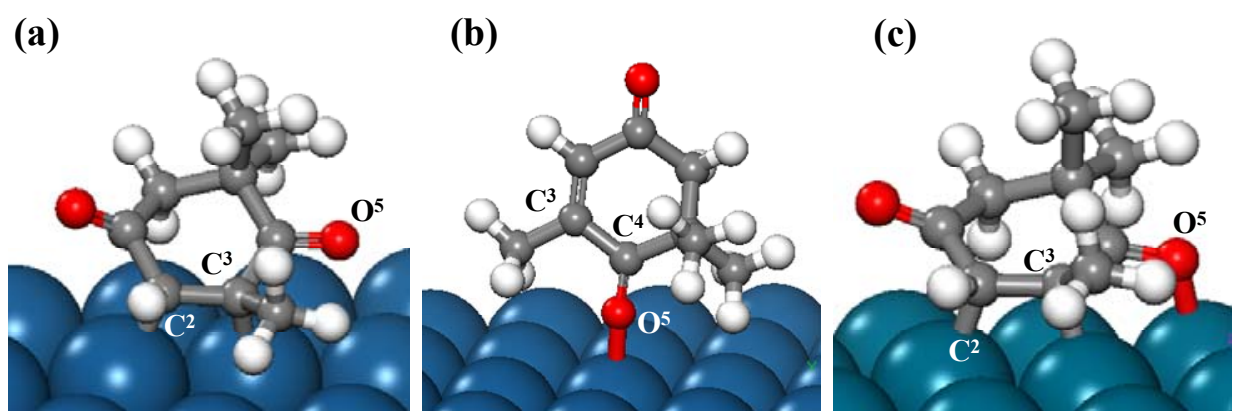

Figure 3.8 DFT-calculated adsorption structures of ketoisophorone (KIP). (a) Adsorption via $\mathrm{C}^{2}=\mathrm{C}^{3}$ bond over $\mathrm{Pt}(111)$ surface; (b) via the oxygen $\mathrm{O}^{5}$ of $\mathrm{C}^{4}=\mathrm{O}^{5}$ group over $\mathrm{Pt}(111)$; (c) via the $\mathrm{C}^{2}=\mathrm{C}^{3}$ bond and the oxygen $\mathrm{O}^{5}$ over $\operatorname{Pd}(111)$ surface.

Table 3.3 DFT-calculated adsorption energies $\left(\mathrm{kJ} \mathrm{mol}^{-1}\right)$ for the reactants, intermediates and products of KIP hydrogenation on $\operatorname{Pt}(111)$ and $\operatorname{Pd}(111)$ surfaces.

\begin{tabular}{ccc}
\hline Specie & $\operatorname{Pt}(111)$ & $\operatorname{Pd}(111)$ \\
\hline KIP & $-18^{\mathrm{a}},-15^{\mathrm{b}},-16^{\mathrm{c}}$ & $-42^{\mathrm{d}},-21^{\mathrm{c}}$ \\
$\mathrm{m} 2$ & -81 & -90 \\
$\mathrm{~m} 3$ & -118 & -119 \\
$\mathrm{~m} 5$ & -56 & -100 \\
$\mathrm{~m} 6$ & -41 & -54 \\
phorenol & -23 & -27 \\
SK & -12 & -15 \\
UA & -20 & -40 \\
\hline
\end{tabular}

${ }^{a}$ Adsorbed via the $\mathrm{C}^{2}=\mathrm{C}^{3}$ bond; ${ }^{\mathrm{b}}$ via the oxygen $\mathrm{O}^{5}$ of $\mathrm{C}^{4}=\mathrm{O}^{5}$ group; ${ }^{\mathrm{c}}$ via the oxygen $\mathrm{O}^{6}$ of $\mathrm{C}^{1}=\mathrm{O}^{6}$ group;

${ }^{d}$ via the $\mathrm{C}^{2}=\mathrm{C}^{3}$ bond and the oxygen $\mathrm{O}^{5}$ center. 


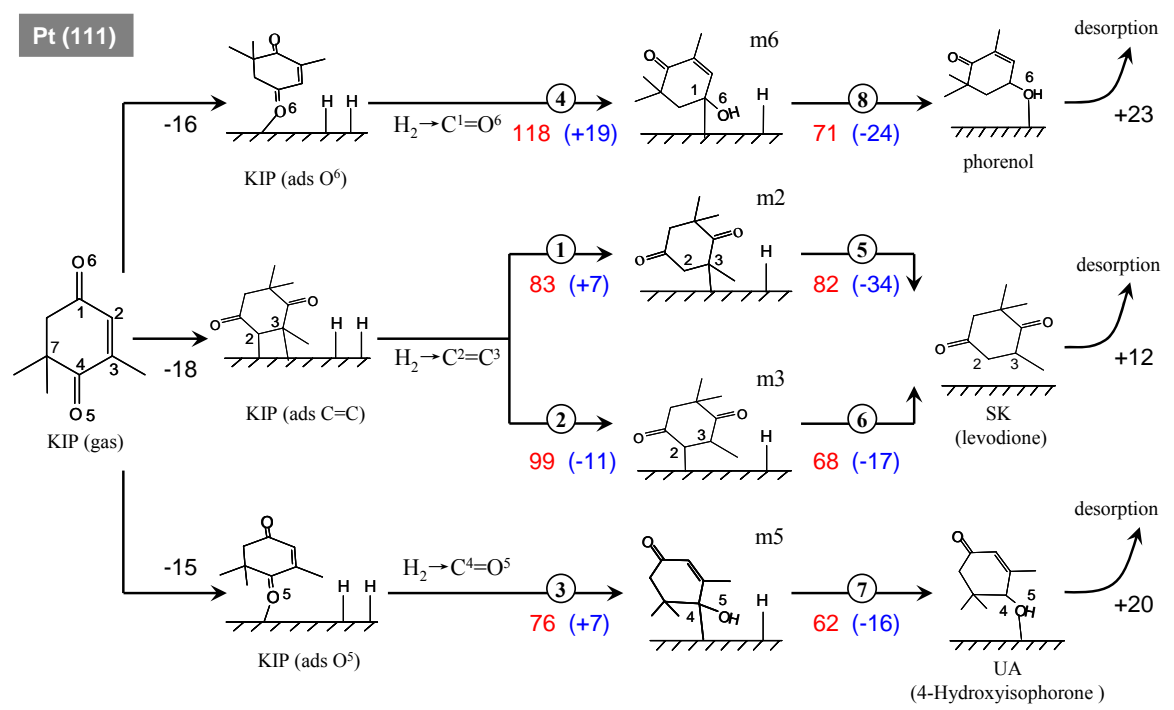

(a)

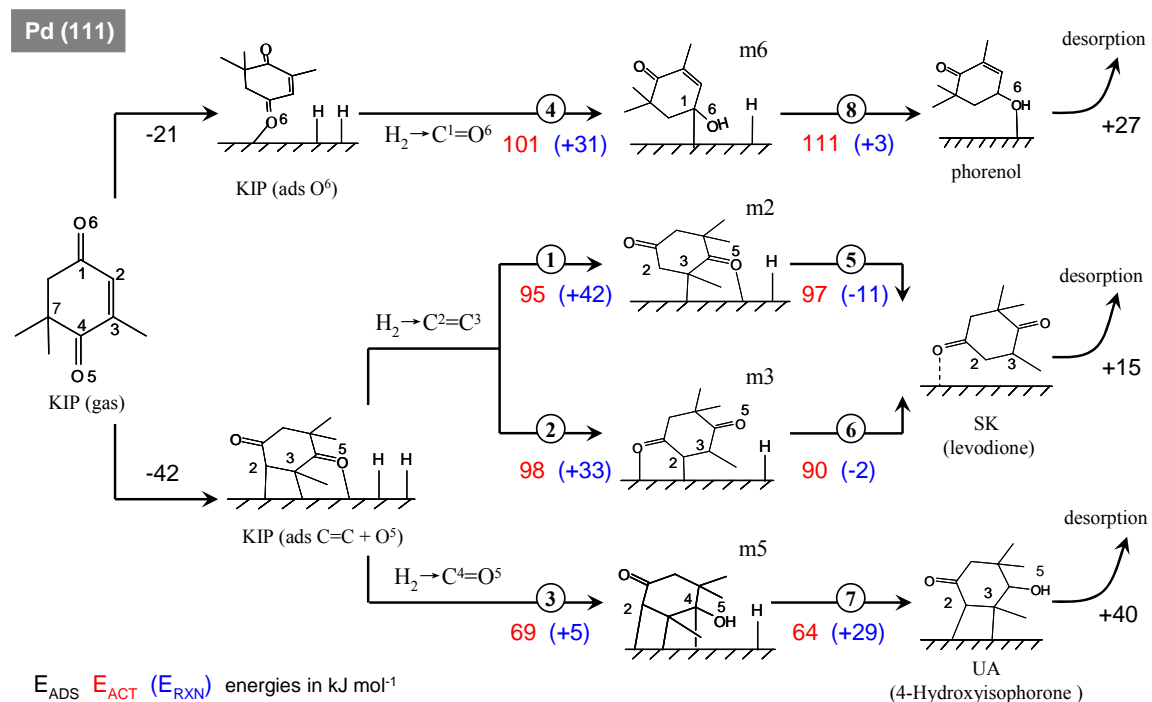

(b)

Figure 3.9 Competitive reaction pathways for the hydrogenation of ketoisophorone over (a) $\operatorname{Pt}(111)$ and (b) $\operatorname{Pd}(111)$ surfaces. The first hydrogenation intermediates $\mathrm{m} 2, \mathrm{~m} 3, \mathrm{~m} 5$ and $\mathrm{m} 6$ are named after the site at which the $\mathrm{H}$ adds to. Pathways 1-4 indicate the first hydrogenation step, and paths 5-8 refer to the second hydrogenation reactions. Red numbers indicate the activation barriers $\left(\mathrm{kJ} \mathrm{mol}^{-1}\right)$ and blue numbers between brackets indicate the reaction energies. Adsorption and desorption energies are shown in black.

The adsorption energies for all of the surface reaction species examined, as well as the activation barriers and the reaction energies of the competitive pathways for the hydrogenation of ketoisophorone over the $\operatorname{Pt}(111)$ and $\operatorname{Pd}(111)$ surfaces are summarized in Table 3.3 and in Figure 3.9. The first hydrogen atom can add to either the $\mathrm{C}^{2}$ or $\mathrm{C}^{3}$ carbon atoms of the $\mathrm{C}^{2}=\mathrm{C}^{3}$ bond, or to the oxygen $\left(\mathrm{O}^{5}\right)$ atom of the sterically-crowded $\mathrm{C}^{4}=\mathrm{O}^{5}$ bond, or to the oxygen $\left(\mathrm{O}^{6}\right)$ atom of the unhindered $\mathrm{C}^{1}=\mathrm{O}^{6}$ bond, to form the $\mathrm{m} 2, \mathrm{~m} 3, \mathrm{~m} 5$ and $\mathrm{m} 6$ intermediates 
shown in Figure 3.9 via path 1, 2, 3 and 4 respectively. The addition of the second $\mathrm{H}$ atom to either the partially saturated $\mathrm{C}=\mathrm{C}$ or $\mathrm{C}=\mathrm{O}$ bond produces the saturated ketone ( $\mathrm{SK}$, levodione) or unsaturated alcohol (UA, 4-hydroxyisophorone) or the byproduct phorenol. The hydrogenation at the carbon $\mathrm{C}^{4}$ in the adsorbed state of Figure $3.8 \mathrm{~b}$ is highly endothermic at over $90 \mathrm{~kJ} \mathrm{~mol}^{-1}$ requiring an activation barrier that is greater than $130 \mathrm{~kJ} \mathrm{~mol}^{-1}$. As a result of the unfavorable energetics, we have not included this step or the subsequent steps along this route in Figure 3.9. The binding energies for the reactant, intermediates and products of KIP were found to be stronger on Pd surface than on Pt surface, as indicated by the energies reported in Table 3.3. This is reflected by the smaller distance between the atoms of the anchoring groups and the metal surfaces. Similar to the case with MVK [13], desorption of the SK of KIP was calculated to require lower energy when compared to the UA over both $\mathrm{Pt}(111)$ and $\mathrm{Pd}(111)$ surfaces.

The methyl substituent at the $\mathrm{C}^{3}$ site and the $\mathrm{C}^{1}=\mathrm{O}^{6}$ group attached to the $\mathrm{C}^{2}$ site were found to limit access to the $C^{3}$ and $C^{2}$ sites and increase the barriers for hydrogenating the $C^{2}=C^{3}$ bond on both the $\operatorname{Pt}(111)$ and $\operatorname{Pd}(111)$ surfaces. This is reflected in reaction energetics reported in Figure 3.9 which indicate that the activation barrier for the first hydrogen addition to the $\mathrm{C}^{2}$ carbon is rather high at $83 \mathrm{~kJ} \mathrm{~mol}^{-1}$ on $\operatorname{Pt}(111)$ and $95 \mathrm{~kJ} \mathrm{~mol}^{-1}$ on $\mathrm{Pd}(111)$ surfaces. The barriers to hydrogenate the substituted carbon $\mathrm{C}^{3}$ are even higher $\left(99 \mathrm{~kJ} \mathrm{~mol}^{-1}\right.$ on Pt, 98 $\mathrm{kJ} \mathrm{mol}^{-1}$ on Pd) than that of carbon $\mathrm{C}^{2}$.

The multiple alkyl substitutions at the $\mathrm{C}^{3}$ and $\mathrm{C}^{7}$ carbon sites that are adjacent to the $\mathrm{C}^{4}=\mathrm{O}^{5}$ bond act to increase the barriers to hydrogenate the $\mathrm{C}^{3}$ site, and weaken the interactions with the surface and promote hydrogen addition to the $\mathrm{O}^{5}$ sites of the adjacent $\mathrm{C}^{4}=\mathrm{O}^{5}$ carbonyl. The activation barrier to add the first hydrogen to the oxygen $\mathrm{O}^{5}$ of the sterically-crowded $\mathrm{C}^{4}=\mathrm{O}^{5}$ bond was calculated to be the lowest barrier for all initial hydrogen additions at $76 \mathrm{~kJ} \mathrm{~mol}^{-1}$ on Pt (111) (path 3 in Figure 3.9a). This first step is also favored over $\operatorname{Pd}(111)$ surface with a barrier of only $69 \mathrm{~kJ} \mathrm{~mol}^{-1}$, which is significantly lower than that for hydrogenating the $\mathrm{C}^{2}=\mathrm{C}^{3}$ bond or the unhindered $\mathrm{C}^{1}=\mathrm{O}^{6}$ carbonyl group.

In contrast to ease of hydrogenating the sterically crowded $\mathrm{C}^{4}=\mathrm{O}^{5}$ bond, the hydrogenation of the unhindered carbonyl $\mathrm{C}^{1}=\mathrm{O}^{6}$ was found to be rather difficult over both the $\mathrm{Pt}(111)$ and $\mathrm{Pd}(111)$ surfaces, as the barriers to add the first hydrogen were calculated to be rather high $\left(118 \mathrm{~kJ} \mathrm{~mol}^{-1}\right.$ on Pt, and $101 \mathrm{~kJ} \mathrm{~mol}^{-1}$ on Pd). These results correlate well to our previous findings of MVK and benzalacetone over Pt surfaces [13] that the direct substitution at $\mathrm{C}=\mathrm{C}$ bond will increase the barriers to hydrogenate at these sites but will promote the hydrogenation of the neighboring carbonyl. The results also help to rationalize experimental observations that indicate that the sterically-hindered $\mathrm{C}=\mathrm{O}$ bond in KIP is selectively hydrogenated while the unhindered carbonyl group is left untouched $[54,91]$. 

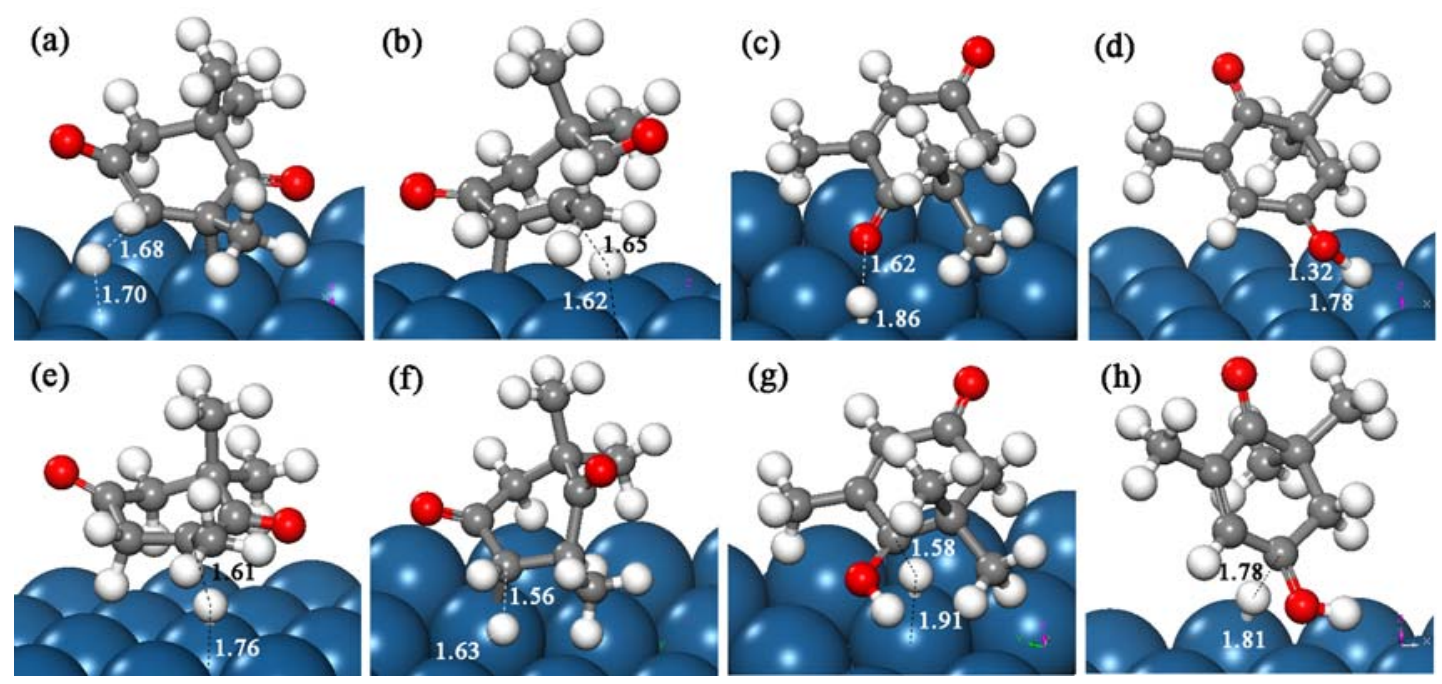

Figure 3.10 Transition states for the reactions of: (a) $\mathrm{KIP} \rightarrow \mathrm{m} 2$; (b) $\mathrm{KIP} \rightarrow \mathrm{m} 3$; (c) $\mathrm{KIP} \rightarrow \mathrm{m} 5$; (d) $\mathrm{KIP} \rightarrow \mathrm{m} 6$; (e) $\mathrm{m} 2 \rightarrow \mathrm{SK} ;(\mathrm{f}) \mathrm{m} 3 \rightarrow \mathrm{SK} ;(\mathrm{g}) \mathrm{m} 5 \rightarrow \mathrm{UA}$; (h) $\mathrm{m} 6 \rightarrow$ phorenol in KIP hydrogenation on Pt (111). Bond lengths are in $\AA$.
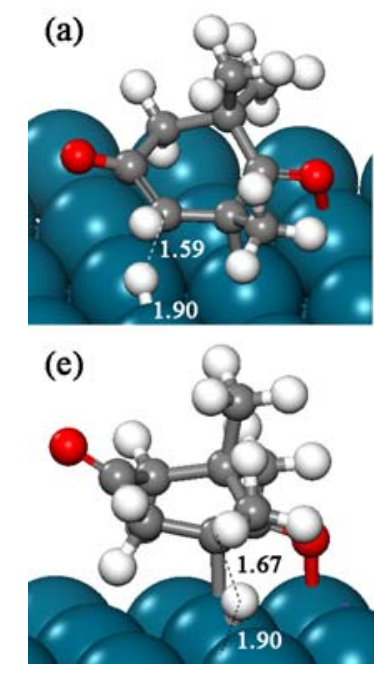
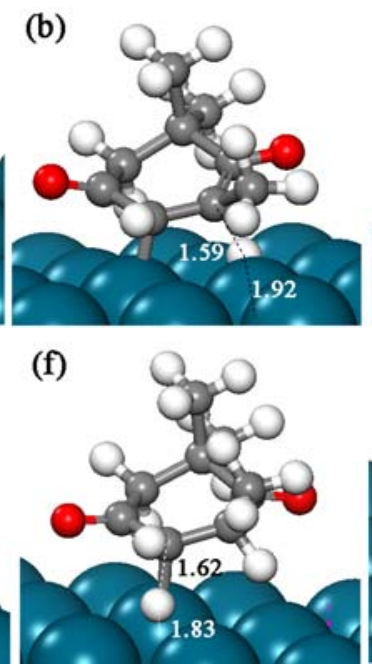

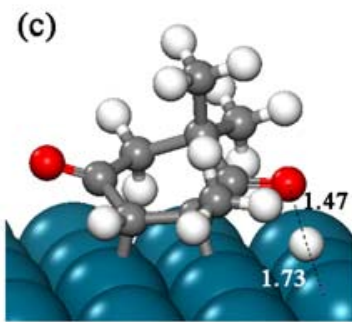

(g)

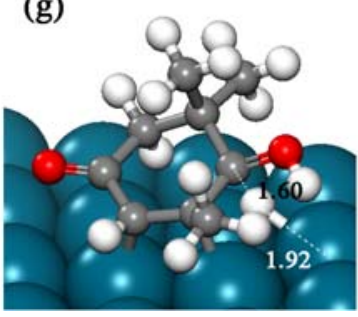

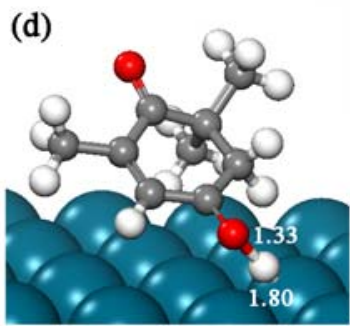

(h)

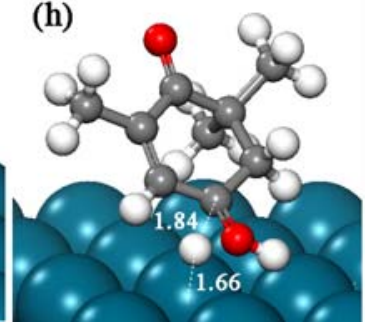

Figure 3.11 Transition states for the reactions of: (a) $\mathrm{KIP} \rightarrow \mathrm{m} 2$; (b) $\mathrm{KIP} \rightarrow \mathrm{m} 3$; (c) $\mathrm{KIP} \rightarrow \mathrm{m} 5$; (d) $\mathrm{KIP} \rightarrow \mathrm{m} 6$; (e) $\mathrm{m} 2 \rightarrow \mathrm{SK}$; (f) $\mathrm{m} 3 \rightarrow \mathrm{SK}$; (g) m5 $\rightarrow \mathrm{UA}$; (h) m6 $\rightarrow$ phorenol in KIP hydrogenation on Pd (111). Bond lengths are in $\AA$.
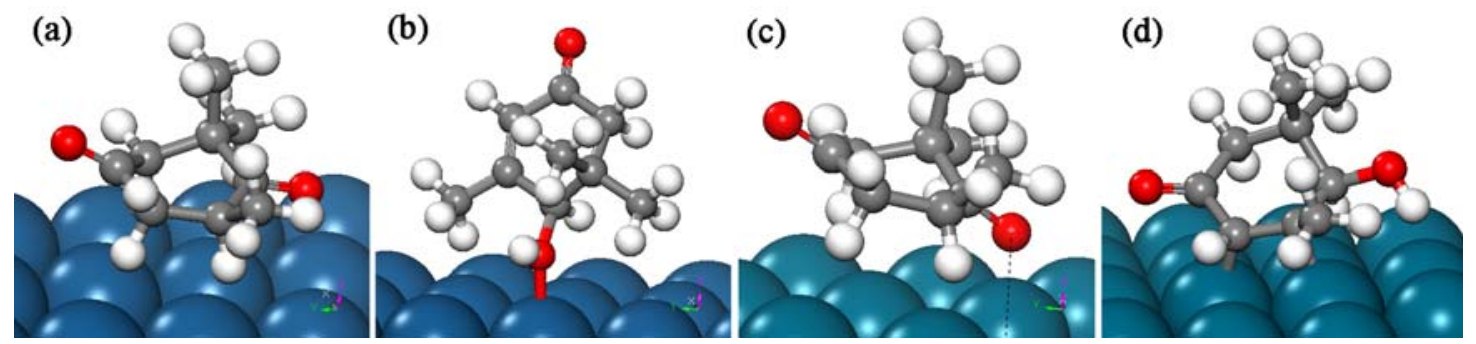

Figure 3.12 Adsorption structures for the products of KIP hydrogenations. (a) SK on Pt(111); (b) UA on Pt(111); (c) SK on Pd(111); (d) UA on Pd(111). Corresponding adsorption energies are referred to Table 3.3. 


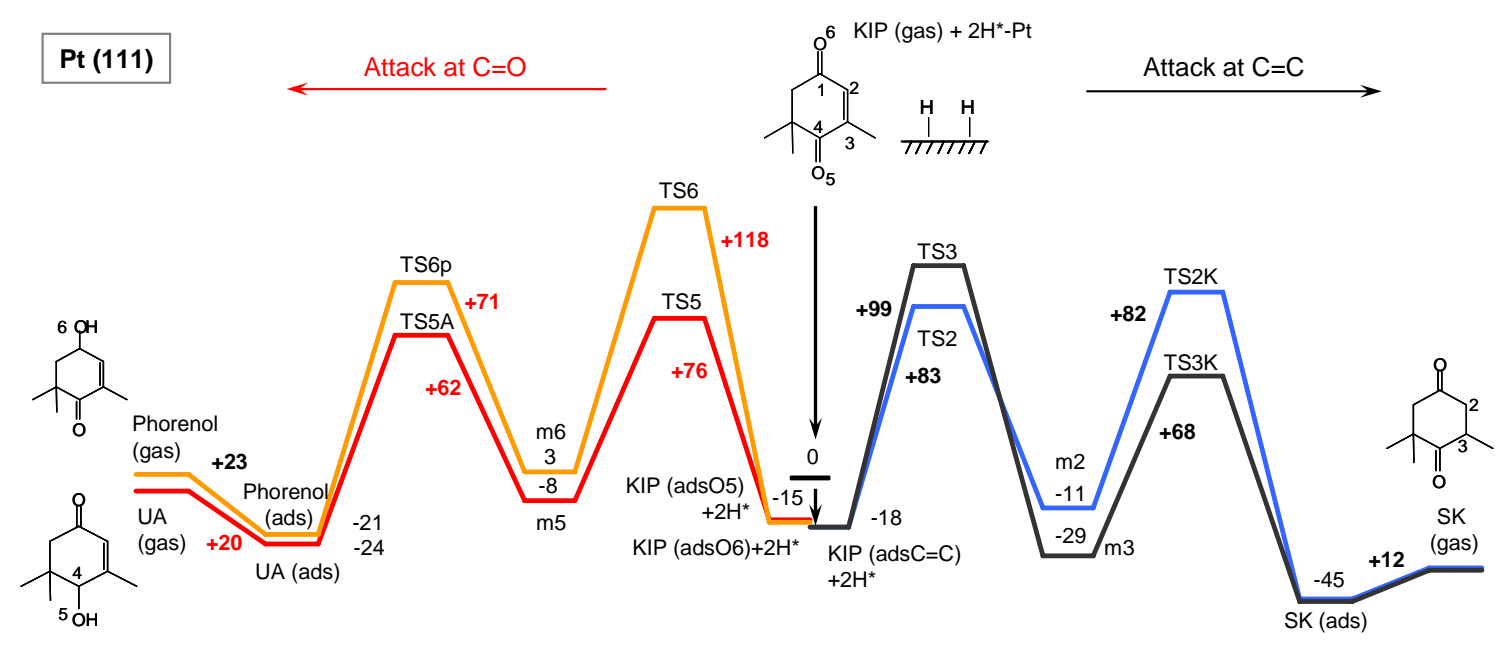

(a)

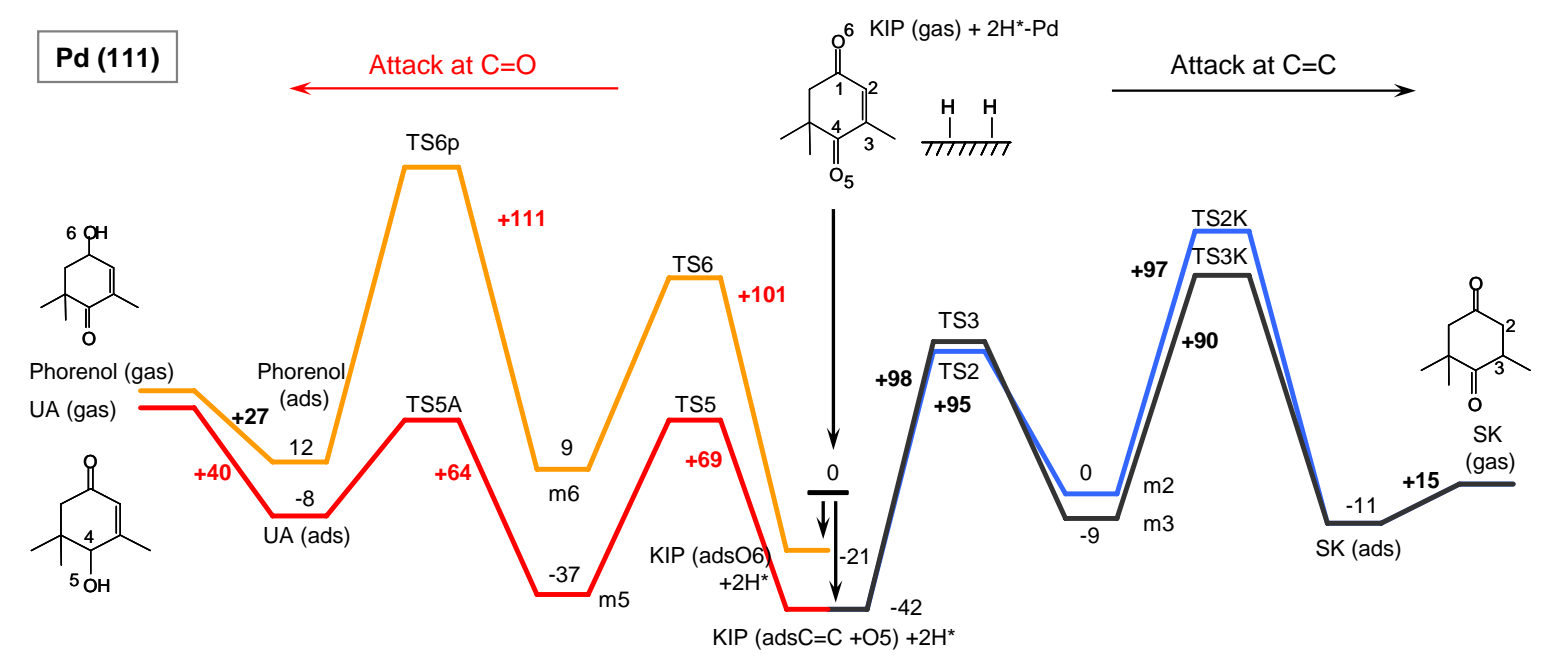

(b)

Figure 3.13 Potential energy profiles of the ketoisophorone hydrogenation on (a) $\operatorname{Pt}(111)$ and (b) $\operatorname{Pd}(111)$. Reaction energies and activation barriers are in $\mathrm{kJ} \mathrm{mol}^{-1}$.

The potential energy profiles for the reactions pathways involved in the hydrogenations of KIP over Pt(111) and $\operatorname{Pd}(111)$ surfaces are plotted in Figure 3.13. $\operatorname{Pt}(111)$ was predicted to have a lower selectivity to unsaturated alcohol (4-hydroxyisophorone) as compared to that on the Pd surface. The formation rates for the unsaturated alcohol and saturated ketone on Pt were thus similar as the hydrogenation of the $\mathrm{C}^{4}=\mathrm{O}^{5}$ and $\mathrm{C}^{2}=\mathrm{C}^{3}$ bonds are nearly equal as shown in Figure 3.13. The addition of the first hydrogen to the $\mathrm{O}^{5}$ site of the $\mathrm{C}^{4}=\mathrm{O}^{5}$ carbonyl is $76 \mathrm{~kJ} \mathrm{~mol}^{-1}$ whereas the addition of hydrogen to the $\mathrm{C}^{2}$ site of the unsaturated $\mathrm{C}^{2}=\mathrm{C}^{3}$ bond is $83 \mathrm{~kJ}$ $\mathrm{mol}^{-1}$. The $\mathrm{m} 5$ and $\mathrm{m} 2$ intermediates that form are likely quasi-equilibrated. This agrees with the experimental observation that $\mathrm{Pt} / \mathrm{Al}_{2} \mathrm{O}_{3}$ produces almost the same amount of UA and SK (levodione) [54, 91]. Both the 
primary UA and SK products are weakly held to Pt surface (-12 20 $\mathrm{kJ} \mathrm{mol}^{-1}$, Figure 3.12a-b) thus resulting in facile desorption from the surface and preventing further reaction to form the saturated ketone.

The $\mathrm{Pd}(111)$ surface was calculated to be much more selective for the reduction of sterically-hindered $\mathrm{C}^{4}=\mathrm{O}^{5}$, carbonyl to form the unsaturated alcohol (4-hydroxyisophorone) as compared to Pt catalyst. The Pd(111) surface binds the $\mathrm{C}^{4}=\mathrm{O}^{5}$ group more favorably than $\mathrm{Pt}$, and results in much lower activation barriers for both the first and the second hydrogen additions to the $\mathrm{C}^{4}=\mathrm{O}^{5}$ bond (69 and $64 \mathrm{~kJ} \mathrm{~mol}^{-1}$ respectively, Figure 3.13b) as compared to the hydrogen additions to the $\mathrm{C}=\mathrm{C}$ bond $\left(95 \sim 98 \mathrm{~kJ} \mathrm{~mol}^{-1}\right)$. The barriers to add to the $\mathrm{C}=\mathrm{O}$ bond are over $30 \mathrm{~kJ} \mathrm{~mol}^{-1}$ lower than those for the $\mathrm{C}=\mathrm{C}$ bond which will result in markedly higher selectivities to UA over Pd than those found on Pt. This agrees very well with the experimental results which report greater than $90 \%$ selectivities to $\mathrm{UA}$ over $\mathrm{Pd} / \mathrm{Al}_{2} \mathrm{O}_{3}$ vs. $\sim 50 \%$ selectivities for $\mathrm{Pt} / \mathrm{Al}_{2} \mathrm{O}_{3}$ [54, 91]. A comparison of the calculated desorption energies indicate that the saturated ketone (levodione) is much easier to desorb (only 15 $\left.\mathrm{kJ} \mathrm{mol}^{-1}\right)$ from Pd than the unsaturated alcohol $\left(40 \mathrm{~kJ} \mathrm{~mol}^{-1}\right)$ which is consistent with previous calculations for the desorption of the UA and SK products in the hydrogenation of MVK [13]. The more strongly bound UA (4-hydroxyisophorone) is more likely than the SK (levodione) to further hydrogenation to form the fully hydrogenated product, 4-OCH, if the reaction time is long enough $[54,91]$ (depicted in Figure 3.7).

The observation of UA formed during ketoisophorone hydrogenation as compared to the negligible formation of UA in the hydrogenation of aliphatic ketones (such as MVK, IMK, MPO, and benzalacetone) illustrates the importance of multiple substituents and specific substituent-carbonyl positions. Multiple substituents attached to the $\mathrm{C}=\mathrm{C}$ bond of KIP successfully decrease the rate of $\mathrm{C}=\mathrm{C}$ bond hydrogenation and thus enhance the probability of hydrogenating $\mathrm{C}=\mathrm{O}$ bond to desired unsaturated alcohol. The direct substitution of the unsaturated carbons not only increase the barriers to hydrogenate the $\mathrm{C}=\mathrm{C}$ bonds but also act to promote the hydrogenation at the neighboring sterically-crowded $\mathrm{C}=\mathrm{O}$ bond. As shown in Figure 3.14(a), the branching alkyl substitutions around the hindered $\mathrm{C}^{4}=\mathrm{O}^{5}$ carbonyl group help to distribute and stabilize the partial charges at the transition state. The sterically-hindered carbonyl thus shows more charge delocalization and less polarization at transition state, which results in a lower activation barrier for hydrogenation as compared to the unhindered $\mathrm{C}=\mathrm{O}$ group. 


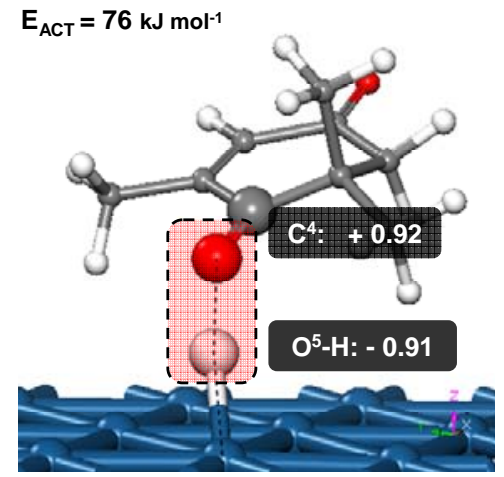

(a)

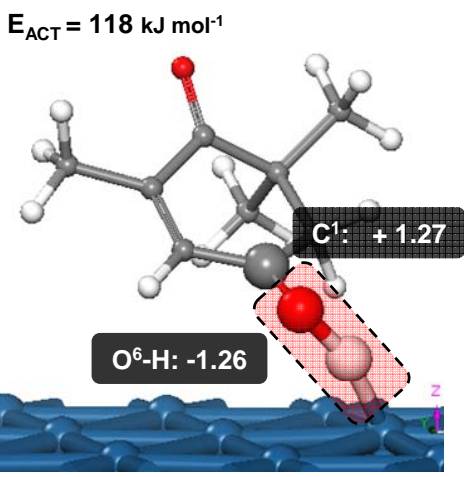

(b)

Figure 3.14 Atomic charges at the transition states for the hydrogen attack on (a) the oxygen $\mathrm{O}^{5}$ of the stericallyhindered carbonyl $\mathrm{C}^{4}=\mathrm{O}^{5}$ bond and (b) on the oxygen $\mathrm{O}^{6}$ of the unhindered $\mathrm{C}^{1}=\mathrm{O}^{6}$ bond over $\mathrm{Pt}(111)$. Charge calculations are performed by Bader methods [100].

\section{CONCLUSIONS}

The hydrogenation of more complex substituted unsaturated ketones, namely benzalacetone and ketoisophorone, were examined over model $\mathrm{Pt}, \mathrm{Pd}$ and $\mathrm{Ru}$ catalysts as there is experimental evidence that at least ketoisophorone can lead to higher selectivities to the unsaturated alcohol. The calculated adsorption energies and the intrinsic reaction energies and activation barriers over model $\operatorname{Ru}(0001), \operatorname{Pd}(111)$ and $\operatorname{Pt}(111)$ surfaces are used to help understand the negligible selectivities in the hydrogenation of benzalacetone to the unsaturated alcohol reported over supported Pt and Ru catalysts as well as the markedly different selectivities found in the hydrogenation of ketosiophorone to unsaturated alcohol over supported Pd and Pt catalysts.

DFT calculated barriers for benzalacetone hydrogenation were significantly higher over $\mathrm{Ru}$ as compared to Pt, which is consistent with the lower experimental TOF reported over supported Ru than Pt. The Pd catalyst tends to be more selective for the reduction of the sterically-hindered carbonyl group in ketosiophorone hydrogenation, leading to higher selectivity to unsaturated alcohol as compared to Pt. The other unhindered carbonyl group is left untouched on both Pt and Pd. The branching alkyl substitutions around the hindered carbonyl group were found to help to distribute and stabilize the partial charges at transition state and lower the barrier. The substitution of single substituent (methyl, aromatic ring) at the $\mathrm{C}=\mathrm{C}$ bond in unsaturated ketones does not appear to change the rate enough to favor the formation of unsaturated alcohols, while the multiple substitutions at the hydrocarbon backbone was found to alter the selectivity. The high selectivity to UA observed during ketoisophorone hydrogenation compared to the complete lack of UA in the hydrogenation of aliphatic ketones such as MVK, IMK, and benzalacetone illustrates the importance of multiple substitutions and the specific substituent positions. 


\section{Chapter 4 - Solvent Effects in Liquid-Phase Hydrogenation Reactions}

\subsection{Effects of Polar and Protic Solvents:}

Hydrogenation of Acrolein in Aqueous and Mixed Alcohol-water Solvents

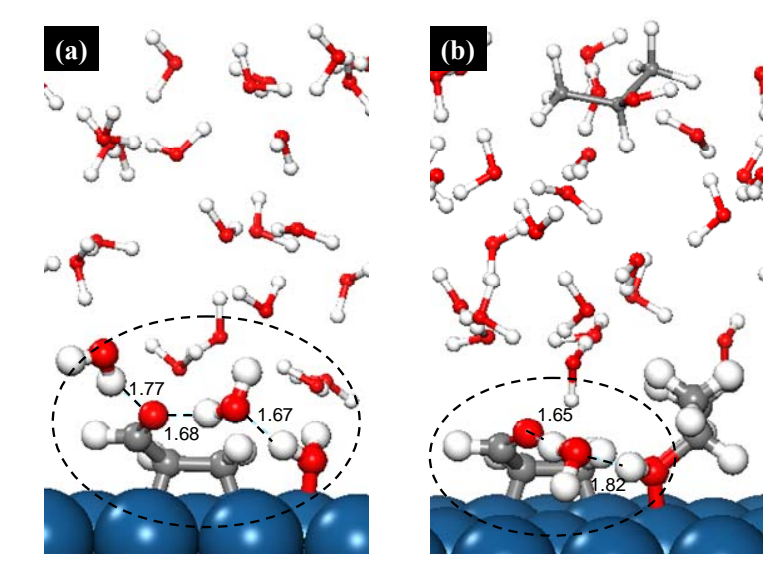

Water and mixed alcohol-water solvents can lower the activation barriers for different metal catalyzed reactions by stabilizing the partial charged transition state and by alter the preferred reaction mechanism in which the solvent molecules can directly participate in the reaction. Theoretical results correlate well with the experimental observed increased selectivity to unsaturated alcohol in 10\% mixed IPA-water solvent, and shed light to the possibility of tailoring catalytic performance through controlling the solvent or the composition of solvents used to carry out the reaction.

\subsection{Effects of Non-Polar Aromatic Solvents:}

Hydrogenation of Benzylacetone in Toluene

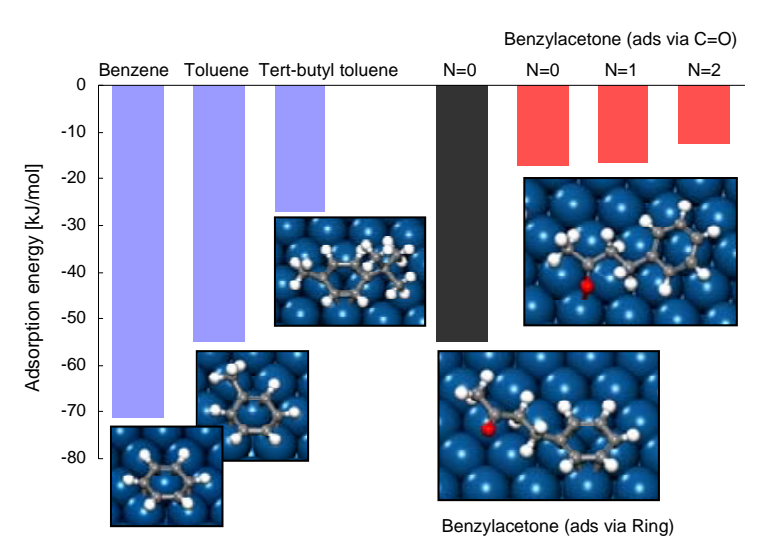

Non-polar aromatic solvents such as toluene can adsorb onto the metal, block the surface sites and thus inhibit the adsorption and ring hydrogenation of benzylacetone. In presence of toluene, the selective conversion of benzylacetone to phenylbutanol increases greatly as the only available path for hydrogenation is via the $\mathrm{C}=\mathrm{O}$ bond. The bulky side chain of tert-butyl toluene limits its ability to efficiently block surface and thus leads to a higher rate but more products from ring hydrogenation of benzylacetone. 


\subsection{Hydrogenation of Acrolein in Aqueous and Mixed Alcohol-water Solvents}

\section{INTRODUCTION}

Many industrially important catalytic hydrogenations of $\alpha, \beta$-unsaturated carbonyl compounds are carried out over supported metal catalysts in solution. The solvents not only provide a media for carrying out the reaction but can influence the reaction rate and selectivity in range of different ways as was reviewd by Singh et al. [2] The solvent can be used to control the polarity of the medium, enhance hydrogen solubility, increase mass transfer, solvate the reactants and products in solution [12], stabilize partially charged transition states [28] and in some cases co-catalyzed particular reactions by directly participating in the mechanism. Polar protic solvents, such as water and alcohols can readily carry out the hydrogenation of $\alpha, \beta$-unsaturated aldehydes and ketones over metal surfaces where the increased hydrogen bonding and possible proton transfer paths in the transition state can enhance the reactivity of $\mathrm{C}=\mathrm{O}$ groups over those of the $\mathrm{C}=\mathrm{C}$ bonds [29]. Previous studies suggest that protic polar solvents can activate and accelerate the hydrogenation of the $\mathrm{C}=\mathrm{O}$ bond in cinnamaldehyde at $50^{\circ} \mathrm{C}$ and 1 bar whereas non-polar solvents favored hydrogenation of $\mathrm{C}=\mathrm{C}$ bond over $\mathrm{Pd} / \mathrm{C}$, $\mathrm{Pt} / \mathrm{C}$ and $\mathrm{Co} / \mathrm{Al}_{2} \mathrm{O}_{3}[30]$.

The hydrogenation of carbonyl groups to form alcohols is thought to be promoted by the presence of water. Akpa et al. [31], for example, reported that the hydrogenation of saturated ketone (methyl ethyl ketone, MEK) over Ru is significantly faster when carried out in water than when carried out in methanol, isopropyl alcohol (IPA) or heptane. It has been proposed that the protic solvent can influence the kinetics of reactions that proceed in reactions involving polar or charged transition states by stabilizing the resulting charges $[28,31,32$, 53]. This has been confirmed in theoretical studies which show that water can offer increased hydrogen bonding in the transition state which can lower the activation energy by $20 \mathrm{~kJ} \mathrm{~mol}^{-1}[31,67]$ or by solvating the anions and cations that form in heterolytic reactions. Water can not only influence the activation energies of elementary steps but in some cases can also directly participate in the reaction and behave as a co-catalyst offering new and preferred reaction channels. Water can efficiently shuttle protons and when coupled with the metal surface offers a conduit for efficiently carrying out proton-coupled-electron transfer paths which are pervasive throughout nature. We have shown previously that proton transfer can readily occur through a chain of water molecules in the hydrogenation of saturated ketones [31, 67], which may help to rationalize the increased activity and selectivity for the hydrogenation of ketones in water.

As was discussed, water is significantly better than alcohols and alkanes in the hydrogenation of carbonyls, as it can more efficiently stabilize the transition states and allow for facile proton shuttling paths via the formation 
of very effective 3D hydrogen-bonding networks [31, 67]. Recent studies indicate that the mixed alcohol/water solvents were reported to enhance the hydrogenation of 2-butanone over Pd catalysts, as compared to pure alcohol solvents [101]. A mixed methanol/water solvent containing $18 \%$ water was shown to increase the reaction rate by almost $50 \%$ over that in pure methanol in the hydrogenation of o-nitrotoluene over $\mathrm{Pd} / \mathrm{C}$ [102]. Recent studies by Hardacre and Thompson showed that the rates of reaction depend on the mixture of the solvents used in the hydrogenation of citral over $5 \% \mathrm{Pt} / \mathrm{SiO}_{2}$ [54]. As shown in Figure 4.1, water accelerates the hydrogenation most among the pure solvents; and the mixed solvent systems, especially the mixture of IPA in $\mathrm{H}_{2} \mathrm{O}$ in a ratio of $1: 9$ (IPA\%=10\%), increased both the rate and selectivity compared to pure solvents. The selectivity to unsaturated alcohols was $96 \%$ with $100 \%$ conversion of citral in the mixed $10 \%$ IPA-water solvents [54].
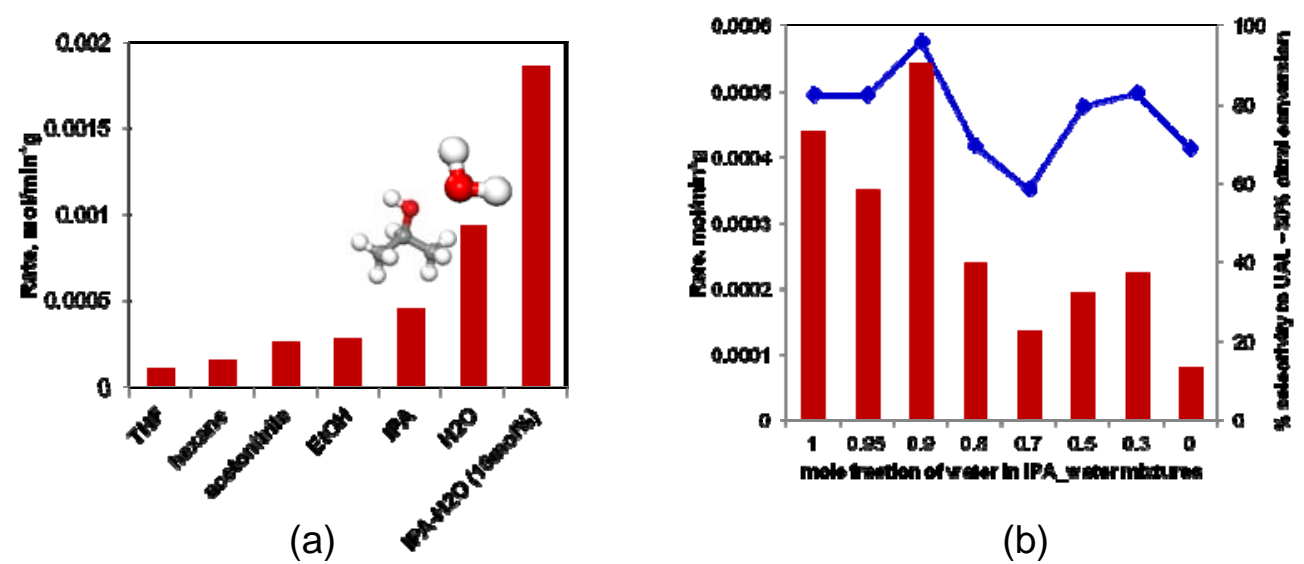

(b)

Figure 4.1 (a) Reaction rates for 2-butanone hydrogenation in different pure solvents over $5 \% \mathrm{Pt} / \mathrm{SiO}_{2}$ at $\mathrm{T}=303 \mathrm{~K}$ [54]. (b) Reaction rates and selectivity to unsaturated alcohol product of citral hydrogenation in the mixed IPA- $\mathrm{H}_{2} \mathrm{O}$ systems over $5 \% \mathrm{Pt} / \mathrm{SiO}_{2}$ at $\mathrm{T}=373 \mathrm{~K}[54]$.

Initial experimental and theoretical efforts aimed at correlating the hydrogenation activity with factors such as the proton diffusivity, donor/acceptor number [53] or dipole moment [32] of the solvent are rather promising. Despite these initial studies, little is known about how polar and protic solvents influence or co-catalyze metal catalysis. The interaction of water molecules with transition metal surfaces are well described in literature [103-105], but how the hydrogen bonding near the surfaces affects the reaction energetics is unknown. While the catalytic hydrogenation is thought to follow Horiuti-Polanyi mechanism [106] that involves sequential addition of hydrogen to the unsaturated $\mathrm{C}=\mathrm{C}$ or $\mathrm{C}=\mathrm{O}$ bond, it is not known how these elementary steps take place in the presence of a mixed solvent. A systematic study of the solvent effects on all of the different elementary steps involved in the hydrogenation of unsaturated aldehydes and ketones is carried out herein in order to appropriately compare with the measured apparent reaction kinetics. 
In this chapter, we extend our initial studies on the vapor phase hydrogenation of unsaturated aldehydes and ketones and computationally model the hydrogenation of a probe unsaturated aldehyde, acrolein, over $\operatorname{Pt}(111)$ metal surface in presence of solvents with DFT calculations. More specifically, the most favorable configurations of solvents near the metal are determined to understand the nature of the liquid/metal interactions and to explore how the structure and composition of the solvent influence the adsorption behavior and reactivity of the reactants. The plausible mechanisms that control the hydrogenation of acrolein in presence of water as well as the mixed alcohol/water solvent (10\% isopropyl alcohol) are explored to draw a comparison with the reaction mechanisms in vapor phase in order to investigate the influence of water over elementary reaction steps in aqueous phase, as depicted in Figure 4.2. The mixture effects, i.e. the influence of changes in the composition of solvent, are studied by comparing the pure water and the mixed solvent. The possible solvent mediated pathways such as the proton shuttling by solvent [67] or the hydrogenation coupling with the IPA dehydrogenation are examined to elucidate how the pure and mixed protic solvents have influenced the preferred reaction pathways in liquid phase hydrogenation.

\section{METHODOLOGY}

First-principle generalized gradient-corrected periodic density functional theory [107] calculations were used to determine the reaction energies and activation barriers for elementary pathways involved in the hydrogenation of acrolein over a model $\operatorname{Pt}(111)$ surface and examine the effects of the solvents. The $\operatorname{Pt}(111)$ surface was modeled using a $3 \times 3$ unit cell comprised of four metal layers along with $16 \AA$ vacuum spacing normal to the metal surface to separate the metal slabs in the z-direction. The top two metal layers of the slab were allowed to relax to their equilibrium positions, while the bottom two layers were held fixed at their bulkphase lattice positions. The aqueous medium was simulated by filling the vacuum region (16 $\AA$ ) above metal slab within the unit cell with 24 water molecules (4 layers, $6 \mathrm{H}_{2} \mathrm{O}$ per layer) to model a density of water of $1 \mathrm{~g} / \mathrm{cm}^{3}$. The initial water structures on $\mathrm{Pt}$ were taken from previous $30 \mathrm{ps}$ ab initio molecular dynamic simulations carried out for water on $\operatorname{Pt}(111)$ at $298 \mathrm{~K}$ [108]. For the reaction carried out in the mixed isopropyl alcohol (IPA) and water solvent, two IPA and 22 water molecules were used to fill the vacuum region in order to simulate a $10 \%$ IPA-water mixture.

The initial structure of water and the mixed solvent, represented by explicit solvent molecules, were obtained by carrying out via a series of simulated annealing studies using $a b$ initio molecular dynamics (MD) method as implemented in VASP. The MD simulations were performed at $400 \mathrm{~K}$ with a time step of $1.0 \mathrm{fs}$ to obtain an optimized low energy structure for the metal-liquid interface. The heating and constant temperature stages of MD annealing were run for 500 time steps $(0.5 \mathrm{ps})$ respectively and subsequently followed by a slow cooling of the system for 1000 time steps (1ps). A Nose-Hoover thermostat was used to regulate the temperature during 
each run $[109,110]$. The solvent-metal interface structures established from MD simulations were then fully optimized using standard conjugate gradient optimization methods until the forces on all of the atoms were all less than $0.05 \mathrm{eV} / \AA$.

All of the calculations reported herein were carried out using the Vienna ab-initio simulation package (VASP) [68] periodic DFT codes. The Kohn-Sham one-electron equations are solved in the Perdew-Wang 91 form of GGA (generalized gradient approximation) [69] to calculate non-local gradient corrections to the correlation and exchange energies. The electron density is described using plane waves with a kinetic-energy cutoff of 396 $\mathrm{eV}$. The electron-ion interactions in core region were described by Vanderbilt ultrasoft pseudopotentials [70]. The geometry optimizations were carried out via a three-step procedure, where the first step converged the atomic structures to a point where the forces on all atoms were less than $0.10 \mathrm{eV} / \AA$, followed by a second optimization step to reduce the forces to $0.05 \mathrm{eV} / \AA$ where the wave functions were converged to $1 \times 10^{-6} \mathrm{eV}$. Both the first and second steps were performed using $3 \times 3 \times 1$ Monkhorst-Pack k-point mesh [71] to sample the Brillouin zone integration [72], whereas the third and final step was carried out using a $6 \times 6 \times 1 \mathrm{k}$-point mesh as static single-point calculations, to determine the lowest energy structure and ensure a higher level of accuracy on the electronic energy. All the calculations were carried out non-spin-polarized.

All of the calculated transition states were determined by combining the nudged elastic band (NEB) method [74-77] to establish images along the MEP that bracket the transition state and the Dimer method [78-80] to isolate the specific transition state. In the NEB calculations, an initial series of intermediate images were refined using a $3 \times 3 \times 1 \mathrm{k}$-point mesh until the maximum force on each atom decreased to $0.20 \mathrm{eV} / \AA$. The "dimer" was formed by replicating the system with very small distances set between them (set to $0.01 \AA$ ), and rotated through a minimization of the torque to ensure that the dimer is in-line with the MEP. Dimer calculations were carried out by $3 \times 3 \times 1 \mathrm{k}$-point until the maximum atomic forces converged to within $0.05 \mathrm{eV} / \AA$, and finally a $6 \times 6 \times 1 \mathrm{k}$-point was used to determine the energy of the transition state (TS) in a static single-point calculation. For the transition state calculations in the presence of a solvent, the solvent molecules were allowed to move along the reaction coordinate, which enables us to model the possible stabilization of transition state by the solvent molecules.

\section{RESULTS AND DISCUSSION}

\section{1) Vapor Phase Hydrogenation of Acrolein}

We begin by examining the hydrogenation of acrolein in the absence of solvent to draw comparisons with the subsequent studied carried out in the liquid phase to understand the influence of pure or mixed solvents. Acrolein adsorbs on the $\mathrm{Pt}(111)$ in a planar $\eta^{3}$ cis configuration through its oxygen atom and the $\mathrm{C}=\mathrm{C}$ bond 
with an adsorption energy of $96 \mathrm{~kJ} \mathrm{~mol}^{-1}$. The binding geometry and strength agree well with previous theoretical studies of acrolein on close-packed planes of different transition metal surfaces at coverage under 1/9ML $[35,36]$. Chemisorbed acrolein and hydrogen can react via a series of hydrogen addition steps to form the unsaturated alcohol (UOL) as well as the saturated aldehyde (SAL) as demonstrated in Figure 4.2, with activation barriers highlighted in black. The first hydrogen addition is favored for the attack on the oxygen center of the $\mathrm{C}=\mathrm{O}$ bond with a barrier of $40 \mathrm{~kJ} \mathrm{~mol}^{-1}$ which is much lower than the barriers for hydrogenating the $\mathrm{C}=\mathrm{C}$ bond ( 88 and $91 \mathrm{~kJ} \mathrm{~mol}^{-1}$ for paths $\mathrm{c} 1$ and $\mathrm{d} 1$, respectively). The adsorbed hydroxyl intermediate that forms $\mathrm{m} 1$ is also much more stable than the other intermediates thus leading to an exothermic reaction step for $\mathrm{O}-\mathrm{H}$ bond formation. The overall reaction energy for the hydrogen addition to the $\mathrm{C}=\mathrm{C}$ bond is, in contrast, mainly endothermic (Table 4.2). The second hydrogen addition steps take place at $\mathrm{C}=\mathrm{C}$ bond show high barriers again ( 89 and $87 \mathrm{~kJ} \mathrm{~mol}^{-1}$ for paths $\mathrm{c} 2$ and $\mathrm{d} 2$, respectively) while the subsequent addition of $\mathrm{H}$ to the $\mathrm{C}=\mathrm{O}$ bond is easier with a barrier of $75 \mathrm{~kJ} \mathrm{~mol}^{-1}$. The barrier for the attack the oxygen atom (path b2) results again in the lowest barrier $\left(43 \mathrm{~kJ} \mathrm{~mol}^{-1}\right)$ with a mechanism similar to the first hydrogenation step a1.

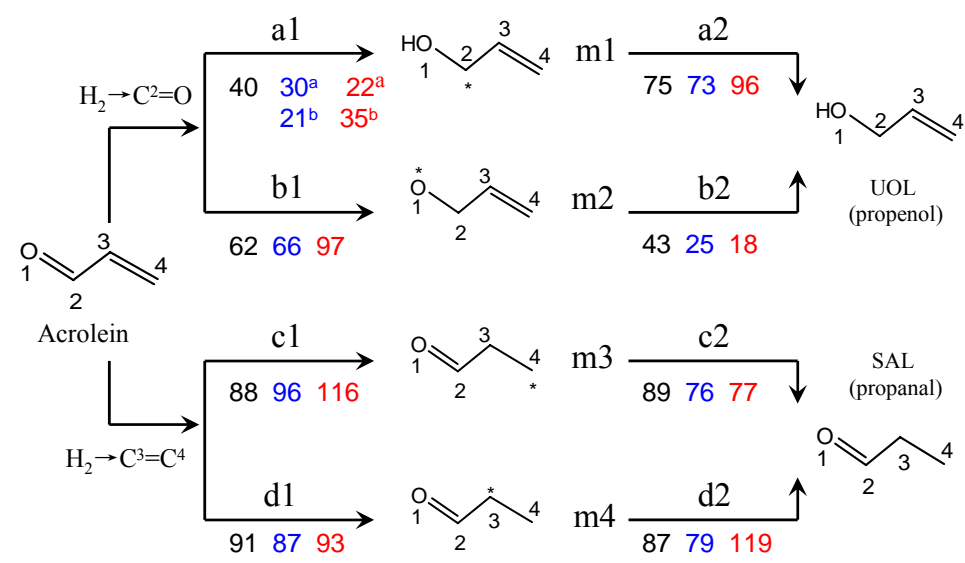

Figure 4.2 Competitive hydrogenation routes of acrolein. The first hydrogenation intermediates $\mathrm{m} 1, \mathrm{~m} 2, \mathrm{~m} 3$ and $\mathrm{m} 4$ are named after the hydrogen addition centers. Pathways al, b1, c1, d1 indicate the elementary reactions of the first hydrogenation step, and $\mathrm{a} 2, \mathrm{~b} 2, \mathrm{c} 2, \mathrm{~d} 2$ refer to the second hydrogenation reactions. Black numbers indicate the activation barriers $\left(\mathrm{kJ} \mathrm{mol}^{-1}\right)$ occurring in vapor phase, blue numbers indicate the activation barriers $\left(\mathrm{kJ} \mathrm{mol}^{-1}\right)$ occurring in water, and red numbers indicate the ones occurring in 10\%IPA-water solvent, respectively. '*' refers to an unsaturated carbon or oxygen that remains bonded to the metal surface after the initial hydrogenation step. $\left({ }^{\mathrm{a}}\right.$ Metal-mediated hydrogenation path. ${ }^{\mathrm{b}}$ Hydrogenation path via proton shuttling.)

As indicated by the energy profile in Figure 4.3, the vapor-phase reaction favors the attack at $\mathrm{C}=\mathrm{O}$ bond to yield unsaturated alcohol (UOL) over the hydrogenation of the $\mathrm{C}=\mathrm{C}$ bond to form the saturated ketone. The strong interaction between the $\mathrm{C}=\mathrm{C}$ bond of $\mathrm{UOL}$ and the $\mathrm{Pt}$ surface, however, hinders its desorption and likely leads to the further hydrogenation to form the unselective saturated alcohol. The $\mathrm{C}=\mathrm{O}$ hydrogenation to form the hydroxyl intermediate $\mathrm{m} 1$ occurs very rapidly. The desorption of unsaturated alcohol product has a barrier of $104 \mathrm{~kJ} \mathrm{~mol}^{-1}$ which is even higher than that of the $\mathrm{C}=\mathrm{C}$ bond hydrogenation $\left(91 \mathrm{~kJ} \mathrm{~mol}^{-1}\right)$. As such, the very 
slow desorption of unsaturated alcohol may give rise to the low selectivity of UOL observed in the vapor phase. This correlates well with the kinetics modeling results [39] that the hydroxyl intermediate was the main adsorbed species during the reaction and the coverage of UOL increase on the surface, as well the experimental observations that over Pt catalyst the selectivity to UOL in acrolein hydrogenation is only $1.6 \%$ whereas $92.6 \%$ to SAL, at low conversion [14]. The saturated aldehyde, that forms from the hydrogenation of the $\mathrm{C}=\mathrm{C}$ bond, on the other hand, binds over $80 \mathrm{~kJ} \mathrm{~mol}^{-1}$ weaker than the UOL and as such can readily desorb from the surface thus preventing the over hydrogenation to the saturated alcohol.

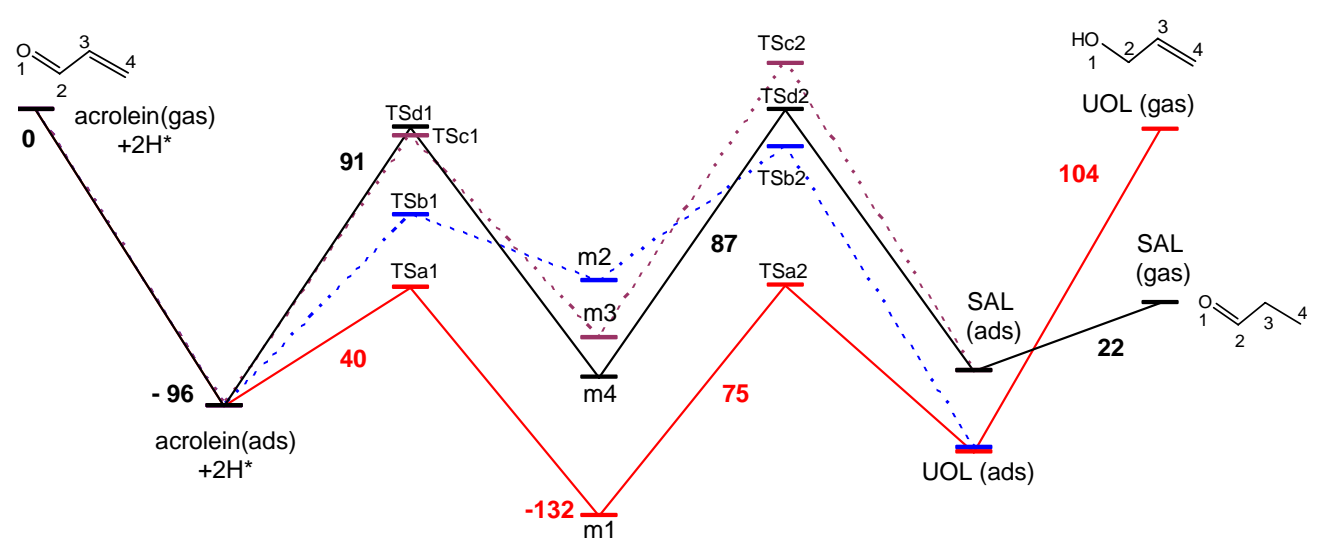

Figure 4.3 The energetic profile of the acrolein hydrogenation on $\operatorname{Pt}(111)$ surface in vapor phase. " $\mathrm{mX}$ " refers to intermediates $\mathrm{m} 1-\mathrm{m} 4$ and "TS" refers to the transition states. Energies are in $\mathrm{kJ} \mathrm{mol}^{-1}$.

\section{2) Metal-mediated Hydrogenation in Solvent Phase}

In order to explore the effects of the reaction environment on the reaction energetics for the hydrogenation of acrolein, we carried out density functional theory calculations to model the reactions that occur in the aqueous and mixed water/alcohol (10\% IPA) solvents. We explicitly explore the reactions that are mediated via the metal and those that are mediated by the solvent.

$A b$ initio molecular dynamics simulations along with simulated annealing simulations were carried out to provide initial configurations of the water molecules near the Pt surface. Water molecules at the surface form a well-established hexagonal bilayer structure that forms an ice structure in the bulk at $0 \mathrm{~K}$. At $298 \mathrm{~K}$, the molecules at the interface show some fluctuations to change in orientation angles with the surface but are still loosely configured in a bilayer like structure. The molecules in the third layer and beyond are much more loosely oriented but with three-dimensional H-bonding (Figure 4.4a). 
In the mixed IPA-water solvent the IPA molecules in the first (closest to surface) layer of the mixed solvent are bound to the surface, with the hydroxyl group participating the chain of hydrogen bonding with water molecules (Figure 4.4b). The MD simulations carried out here provide a very simplified model that takes into account first-order effects to capture the role of explicit solvent molecules at the solvent-metal interface. They do not include the effects of dynamics or temperature [31].

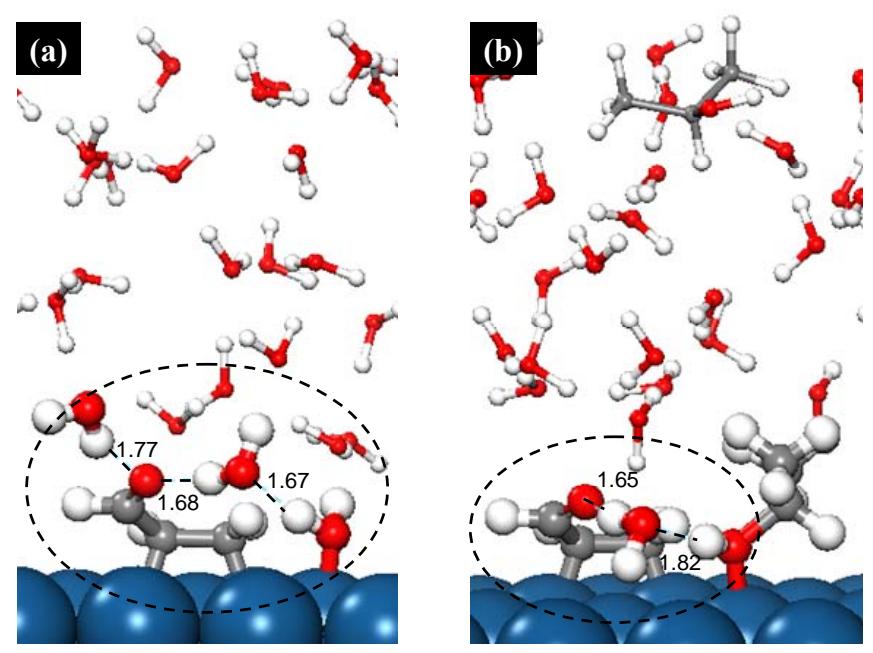

Figure 4.4 DFT optimized adsorption configurations of acrolein at the solvent-metal interface over $\operatorname{Pt}(111)$ in (a) aqueous phase; (b) 10\% mixed IPA-water solvents (distance in $\AA$ ).

Table 4.1 Characteristic bond lengths $(\AA)$ of the adsorbed acrolein at $\operatorname{Pt}(111)$ in vapor phase and in aqueous and mixed IPA-water solvents.

\begin{tabular}{ccccc}
\hline $\begin{array}{c}\text { Bond lengths } \\
(\AA)\end{array}$ & Gas phase & $\begin{array}{c}\text { Vapor adsorbed } \\
\eta^{3} \text { cis }\end{array}$ & $\begin{array}{c}\text { Water } \\
\eta^{2} \text { cis }\end{array}$ & $\begin{array}{c}\text { 10\% IPA-water solvent } \\
\eta^{2} \text { cis }\end{array}$ \\
\hline $\mathrm{O}-\mathrm{C}^{2}$ & 1.23 & 1.27 & 1.26 & 1.24 \\
$\mathrm{C}^{2}-\mathrm{C}^{3}$ & 1.47 & 1.44 & 1.46 & 1.47 \\
$\mathrm{C}^{3}-\mathrm{C}^{4}$ & 1.34 & 1.48 & 1.47 & 1.47 \\
$\mathrm{O}-\mathrm{Pt}$ & - & 2.26 & 3.61 & 3.42 \\
$\mathrm{C}^{2}-\mathrm{Pt}$ & - & 2.65 & 2.84 & 2.89 \\
$\mathrm{C}^{3}-\mathrm{Pt}$ & - & 2.22 & 2.16 & 2.15 \\
$\mathrm{C}^{4}-\mathrm{Pt}$ & - & 2.10 & 1.26 & 2.12 \\
\hline
\end{tabular}

In the presence of water, acrolein adsorbs at the metal-water interface by displacing an adsorbed water molecule from the Pt surface. The acrolein is solvated by 23 water molecules and two water molecules within its local solvation sphere form hydrogen bonds with the oxygen atom of acrolein (Figure 4.4a). The Pt-O distance in aqueous phase is $3.61 \AA$ as compared to $2.26 \AA$ in the vapor phase (Table 4.1). This is due to the stronger interaction of the $\mathrm{C}=\mathrm{O}$ group with the aqueous solvent which occurs through $\mathrm{H}$-bonding than with the 
Pt surface. In the mixed IPA/water solvent the stabilization of acrolein by hydrogen bonding is not as strong as in the case of water, as there is a loss of hydrogen bonding with the mixed solution phase (Figure 4.4b).

Table 4.2 has tabulated DFT-calculated activation barriers and reaction energies in various reaction environments. In the presence of water, the hydroxyl pathway (a1) was found to be more favorable than the alkoxy and alkyl routes as a result of the stronger stabilization of partial-charged transition state to form the $\mathrm{O}-$ $\mathrm{H}$ bond over the $\mathrm{C}-\mathrm{H}$ bond. The hydroxyl intermediate is stabilized by forming hydrogen bonds with water molecules via its $-\mathrm{OH}$ group (Figure 4.6a) and results in an exothermic reaction energy $\left(-39 \mathrm{~kJ} \mathrm{~mol}^{-1}\right)$. At the transition state, the accessibility of the $\mathrm{O}-\mathrm{H}$ reaction center to the bulk solvent (Figure 4.7a) allows it to participate in hydrogen bonding with waters within its local solvation sphere, and leads to extra stabilization over the reactant state. The activation barrier is therefore lowered to $30 \mathrm{~kJ} \mathrm{~mol}^{-1}$ from $40 \mathrm{~kJ} \mathrm{~mol}^{-1}$ in the vapor phase reaction. In the second hydrogenation step, the barrier for hydrogen addition to the oxygen center is again the lowest at $25 \mathrm{~kJ} \mathrm{~mol}^{-1}$ (path b2) which is $\sim 20 \mathrm{~kJ} \mathrm{~mol}^{-1}$ lower than that in vapor phase.

Table 4.2 DFT calculated reaction energies $\left(\mathrm{kJ} \mathrm{mol}^{-1}\right)$ and activation barriers $\left(\mathrm{kJ} \mathrm{mol}^{-1}\right)$ for the hydrogenation of acrolein to propenol (UOL) and propanal (SAL) in vapor phase, water and 10\% IPA-water solvent over Pt(111) surface.

\begin{tabular}{c|cc|cc|cc}
\hline \multirow{2}{*}{ Paths } & \multicolumn{3}{|c|}{ Vapor } & \multicolumn{2}{c|}{ Water } & \multicolumn{2}{c}{$10 \%$ IPA-water solvent } \\
\cline { 2 - 7 } & $\Delta E_{r x n}$ & $E_{a c t}$ & $\Delta E_{r x n}$ & $E_{a c t}$ & $\Delta E_{r x n}$ & $E_{a c t}$ \\
\hline $\mathrm{a} 1$ & -36 & 40 & -39 & $30^{\mathrm{a}}, 21^{\mathrm{b}}$ & -35 & $22^{\mathrm{a}}, 35^{\mathrm{b}}$ \\
$\mathrm{b} 1$ & 41 & 62 & 48 & 66 & 27 & 97 \\
$\mathrm{c} 1$ & 22 & 88 & 38 & 96 & 33 & 116 \\
$\mathrm{~d} 1$ & 10 & 91 & 14 & 87 & 25 & 93 \\
\hline $\mathrm{a} 2$ & 21 & 75 & 13 & 73 & -23 & 96 \\
$\mathrm{~b} 2$ & -54 & 43 & -75 & 25 & -83 & 18 \\
$\mathrm{c} 2$ & -11 & 89 & -18 & 76 & 8 & 77 \\
$\mathrm{~d} 2$ & 2 & 87 & 9 & 79 & 13 & 119 \\
\hline
\end{tabular}

${ }^{\mathrm{a}}$ Metal-mediated hydrogenation path. $\quad{ }^{\mathrm{b}}$ Hydrogenation path via proton shuttling.

The reactions to form the alkoxy and alkyl intermediates (routes b1, c1, d1) involve the addition of hydrogen to the carbon centers, which proceed via transition states that are much less polar than that involved in the addition of hydrogen to the oxygen. As such, there are far less hydrogen bonding stabilization of the $\mathrm{C}-\mathrm{H}$ forming transition states than those that form $\mathrm{O}-\mathrm{H}$ bonds. The activation barrier to add hydrogen to the carbon end of the carbonyl to form the alkoxy intermediate was calculated to be $66 \mathrm{~kJ} \mathrm{~mol}^{-1}$ in water, which is very similar to the value of $62 \mathrm{~kJ} \mathrm{~mol}^{-1}$ for the vapor phase reaction. The $\mathrm{C}=\mathrm{C}$ bond hydrogenation via alkyl path $\mathrm{d} 1$ 
also shows similar barrier to that for vapor phase (Table 4.2) and only a very small stabilization $\left(4 \mathrm{~kJ} \mathrm{~mol}^{-1}\right)$ of the transition over the reactant state resulted by carrying the reaction out in the presence of water, as the number of hydrogen bonds remains the same between the reactant and transition state (Figure 4.7). The barrier to subsequently hydrogenate the hydroxyl to form propenol was calculated to be $73 \mathrm{~kJ} \mathrm{~mol}^{-1}$ in water, which is identical to that found in the vapor phase.

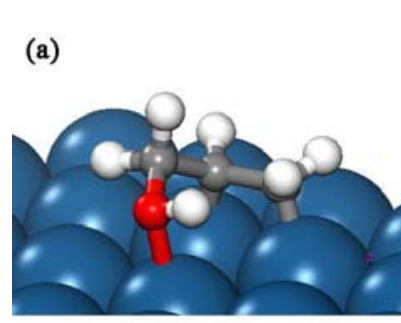

(d)
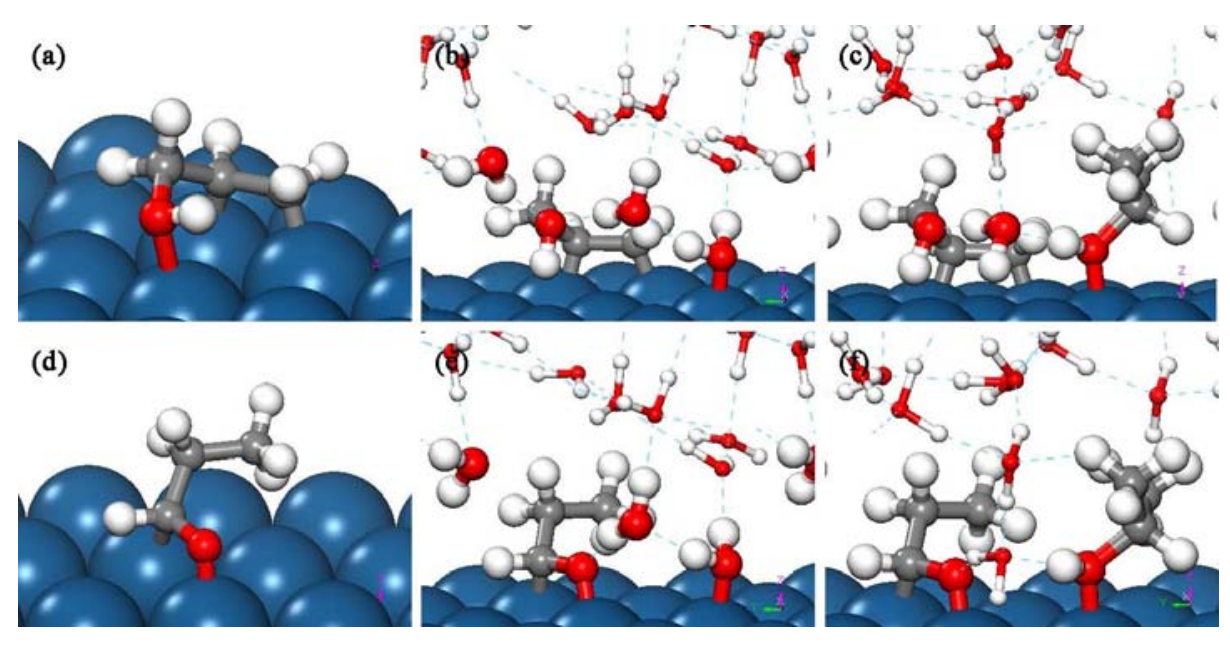

Figure 4.5 Configurations of adsorbed products, unsaturated alcohol in (a) vapor phase; (b) water and (c) mixed IPA/water solvent; and saturated aldehyde in (d) vapor phase; (e) water and (f) mixed solvent.

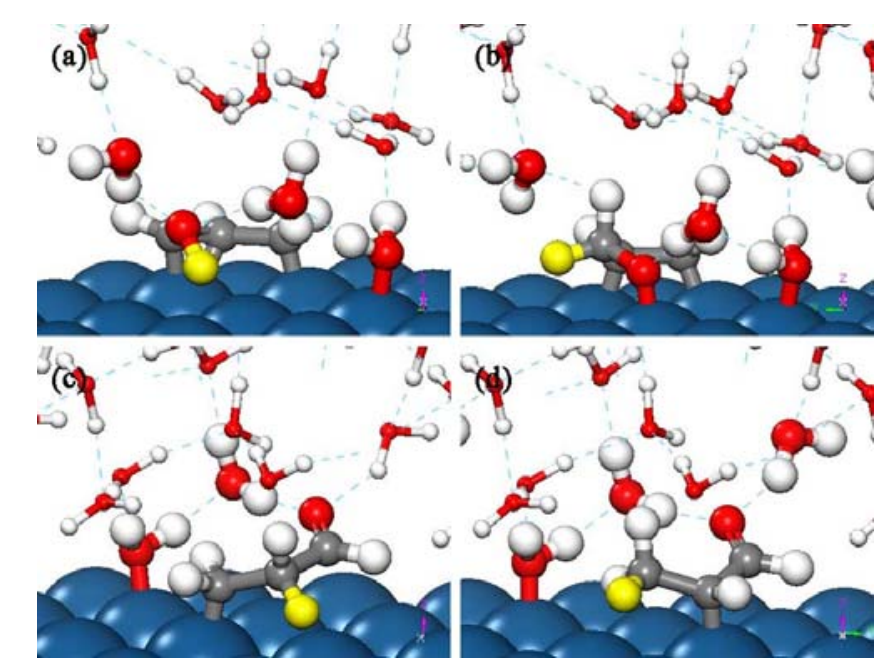

Figure 4.6 Configurations of the intermediates (a) hydroxyl m1; (b) alkoxy m2; (c) alkyl m3 and (d) $\mathrm{m} 4$ of the hydrogenation of acrolein in aqueous phase over $\operatorname{Pt}(111)$.

The same reaction steps are studied in the mixed IPA/water solvent. As observed in the case of water, the activation barrier is lower for the hydroxyl route involving hydrogen addition to the oxygen $\left(22 \mathrm{~kJ} \mathrm{~mol}^{-1}\right)$ than it is for alkoxy and alkyl which involve hydrogen addition to the carbon of the $\mathrm{C}=\mathrm{O}$ and $\mathrm{C}=\mathrm{C}$ bonds, 
respectively. The barrier in the mixed IPA/water system was also found to be lower than that for the vapor phase system $\left(40 \mathrm{~kJ} \mathrm{~mol}^{-1}\right)$. The stabilization by hydrogen bonding of the transition state to form hydroxyl in presence of mixed solvent is not as strong as in the case of water as there is a loss of one hydrogen bond (Figure 4.8a). The activation barrier of the metal-mediated hydroxyl path in mixed solvent, however, is $\sim 10 \mathrm{~kJ}$ $\mathrm{mol}^{-1}$ lower than that found in water.

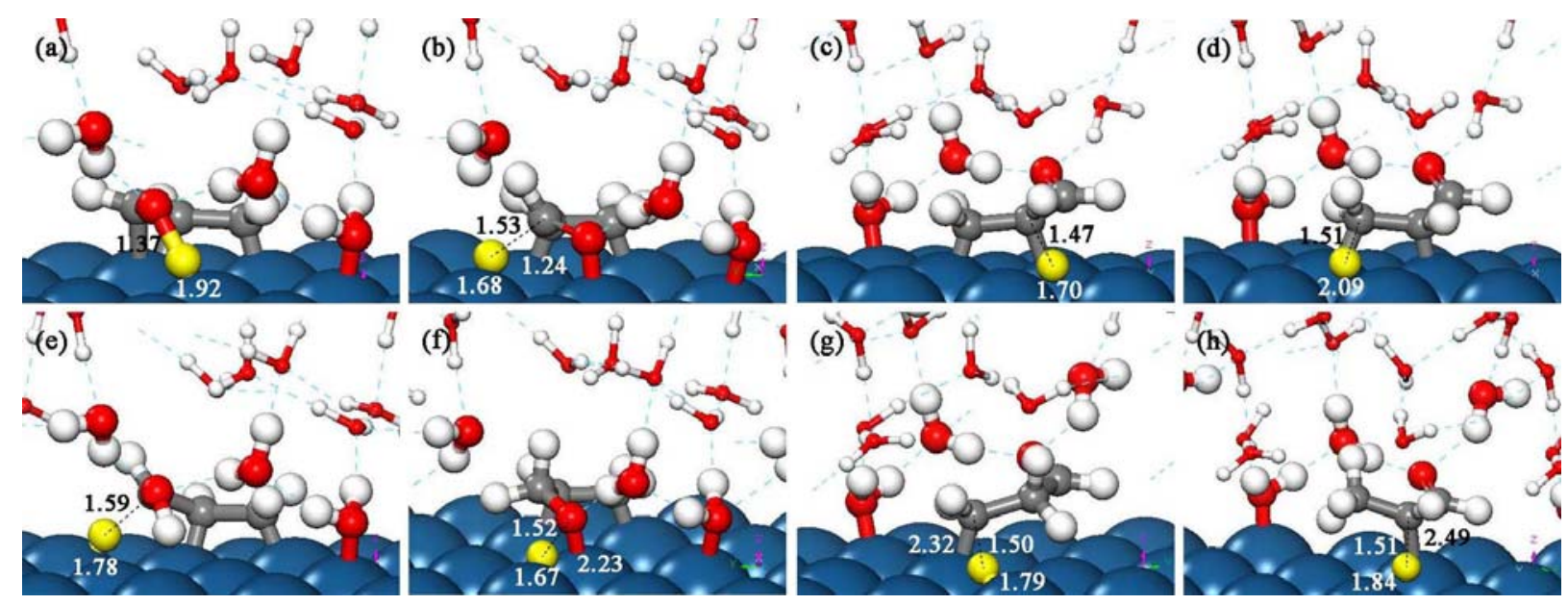

Figure 4.7 DFT-calculated transition state structures for the hydrogenation of acrolein in aqueous phase over Pt(111) for: (a) hydroxyl path a1; (b) alkoxy path b1; (c) alkyl path c1 and (d) path d1; (e) path a2; (f) path b2; (g) path c2; (h) path $\mathrm{d} 2$. The hydrogen atom involved in hydrogenation is shown in yellow (distance in $\AA$ ).

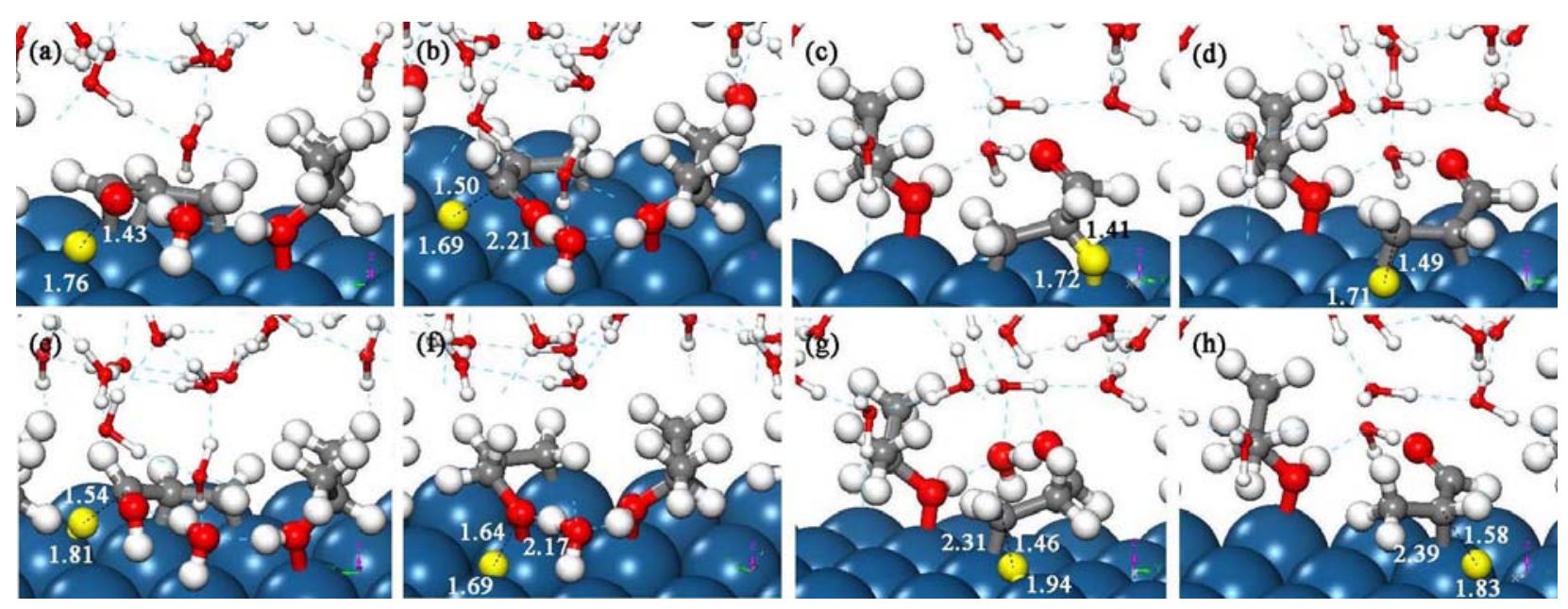

Figure 4.8 DFT-calculated transition state structures for the hydrogenation of acrolein in the mixed IPA/water solvent over Pt(111) for: (a) hydroxyl path a1; (b) alkoxy path b1; (c) alkyl path c1 and (d) path d1; (e) path a2; (f) path b2; (g) path c2; (h) path d2. The hydrogen atom involved in hydrogenation is shown in yellow (distance in $\AA$ ). 
In order to further understand this energy difference upon the changing of solvent structure, Bader charge analysis [100] was carried out to monitor the charge distributions during the formation of hydroxyl in vapor phase, aqueous and 10\% mixed IPA-water solvent. The results are summarized in Figure 4.9a-c. The amount of charge transfer was calculated as the differences in the atomic charge densities in their transition states and their reactant states. In all three reaction media (vapor phase, water, and mixed alcohol-water systems), the hydrogen that dissociates onto the metal surface picks up electrons from the metal and acts as a hydride. As the hydrogen is pulled away from the metal and is added to either the oxygen or the carbon, the electron is transferred back to the metal. If the reaction is carried out in the vapor phase there is a greater polarization of the electrons and less stabilization than that in the cases of solvents. In presence of solvent, more negative charge is delocalized into the metal as well as in the solvent molecules thus resulting in notable stabilizations of the charge delocalization of transition states are obtained. As demonstrated in Figure 4.9c, the transition state of hydroxyl formation in mixed solvent shows less polarization and hence more stable charge distribution upon the hydrogen attack from the metal to the oxygen center, as compared to the case of water. The charges transferred to solvent molecules are also calculated to be more obvious in the mixed solvent. The activation barrier of hydroxyl route is therefore slightly lower in mixed solvent $\left(22 \mathrm{~kJ} \mathrm{~mol}^{-1}\right)$ than that in water $(30 \mathrm{~kJ}$ $\left.\mathrm{mol}^{-1}\right)$.

As reported by Table 4.2, the mixed solvent significantly favors the $\mathrm{C}=\mathrm{O}$ bond hydrogenation via hydroxyl route other than the $\mathrm{C}=\mathrm{C}$ bond reactions, as the latter needs to overcome much higher activation barriers $(\sim 100$ $\mathrm{kJ} \mathrm{mol}^{-1}$ and more). Hydrogenation rates of the $\mathrm{C}=\mathrm{C}$ bond at $375 \mathrm{~K}$ were estimated to be an order of magnitude smaller in the mixed solvent than in water, which enhances the probability of hydrogenating the carbonyl and rationalizing the increased selectivity to unsaturated alcohol in 10\% mixed IPA-water solvent (95\% UOL) as compared to that in pure water (85\% UOL, as observed by experiments [54], Figure $4.1 \mathrm{~b})$.

(a)

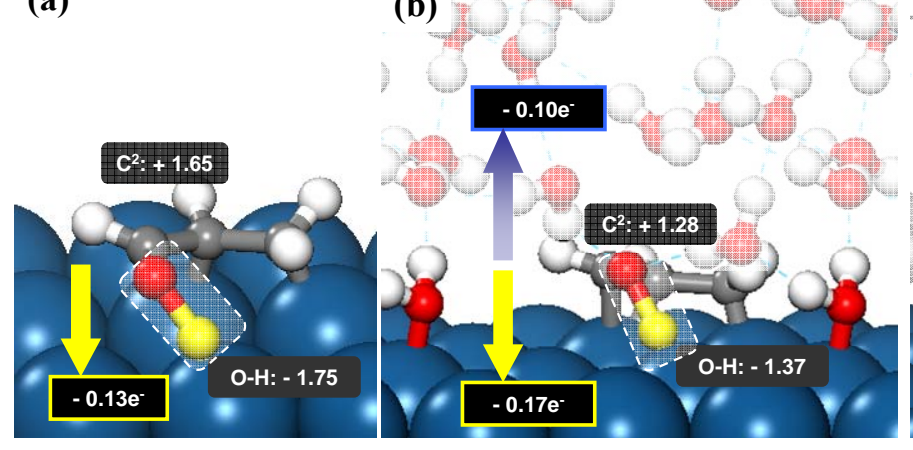

(c)

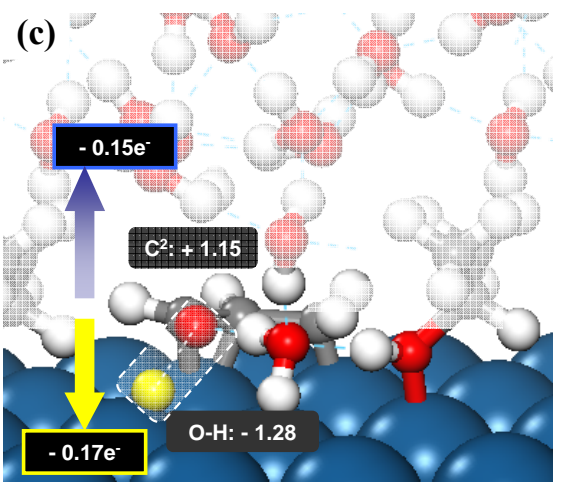

Figure 4.9 Atomic charges and the charge transfer that occurs in the hydrogenation of the oxygen of the $\mathrm{C}=\mathrm{O}$ of acrolein to form hydroxyl intermediate in (a) vapor phase, (b) aqueous phase and (c) 10\% mixed IPA-water solvent. 


\section{3) Solvent-mediated Hydrogenation Pathways}

In addition to its role in stabilizing polar transition states and charged intermediates, protic solvents can also directly participate in the catalyzing the surface reactions via shuttling charged intermediates including protons or hydroxyl groups [31]. Previous theoretical studies have reported on the proton transport in aqueous solutions [31] which involve the formation of hydronium $\mathrm{H}_{3} \mathrm{O}+$ or $\mathrm{Zundel}_{5} \mathrm{O}_{2}{ }^{+}$ion intermediates that allow for facile proton shuttling through networks of hydrogen-bonding solvent molecules. Proton diffusivities are higher in water rather than in the mixed solvents, as water is more hydrophilic and contains more extensive hydrogen bonding. In this section we compare the possible solvent-mediated hydroxyl route in water and mixed solvent to examine whether it can alter the preferred reaction pathways.

The presence of water appears to facilitate proton-coupled-electron transfer (PCET) paths that allow for the hydrogenation of the more electronegative $\mathrm{O}$ centers. As was discussed above, the surface bound hydrogen on most metals is in the form of a hydride. Hydrogenation can proceed by a PCET process in which the electron that formally resides on the $\mathrm{H}$ is transferred to the metal and ultimately into the bound adsorbate complex [31]. The hydrogen is then released into solution as a proton which migrates through a sequence of water molecules to ultimately add together with the electron that transferred to the metal onto the $\mathrm{O}$ of the bound acrolein intermediate as is shown in Figure 4.10a. The $\mathrm{Pt}-\mathrm{H}^{1}$ bond in the transition state is significantly weakened where the proton has formed and becomes part of the hydrogen bonds network, with a new forming $\mathrm{O}-\mathrm{H}^{1}$ bond of $1.43 \AA$. This proton is rapidly shuttled through solution via a chain of water molecules [31] and eventually adds to the oxygen center of acrolein to form the hydroxyl intermediate. At the other end of the chain, the O$\mathrm{H}^{3}$ bond changes from a weak hydrogen bond in the reactant state $(1.84 \AA)$ to the formation of a covalent $\mathrm{O}-\mathrm{H}$ bond $(1.49 \AA)$ in the transition state. This solvent-mediated hydroxyl route is expected to be the dominant pathway for acrolein hydrogenation over $\mathrm{Pt}(111)$ in water, with a barrier of only $21 \mathrm{~kJ} \mathrm{~mol}^{-1}$ which is $\sim 10 \mathrm{~kJ}$ $\mathrm{mol}^{-1}$ lower than that of metal-mediated route. This solvent-mediated mechanism is not expected in the formation of alkoxy intermediate as it can not act to stabilize the addition of hydrogen to the more hydrophobic carbon centers.

The proton shuttling mechanism critically depends on the hydrogen-bonding network that forms to stabilize the transition state and enable facile proton transfer and shuttling [31, 67]. The average number of hydrogen bonds per molecule in the IPA-water mixtures has been reported to decrease with a decreasing water mole fraction $[31,54]$. Although the IPA molecule can participate in the hydrogen bonding network through its hydroxyl group, the presence of the large alkyl group disrupts the highly interconnected hydrogen bonding network between water molecules and subsequently decreases proton transport. A detailed analysis of the charges on the $\mathrm{H}$ and the carbon shown in Figure 4.10b indicate a greater polarization and less stable charge 
distribution within the transition state for the proton shuttling mechanism in mixed solvent (Figure 4.10a) as compared to the case in pure water (Figure 4.10b). Consequently, in mixed solvent the solvent-mediated path $\left(35 \mathrm{~kJ} \mathrm{~mol}^{-1}\right)$ is not as favorable as the metal-mediate path $\left(22 \mathrm{~kJ} \mathrm{~mol}^{-1}\right)$ to form hydroxyl group.
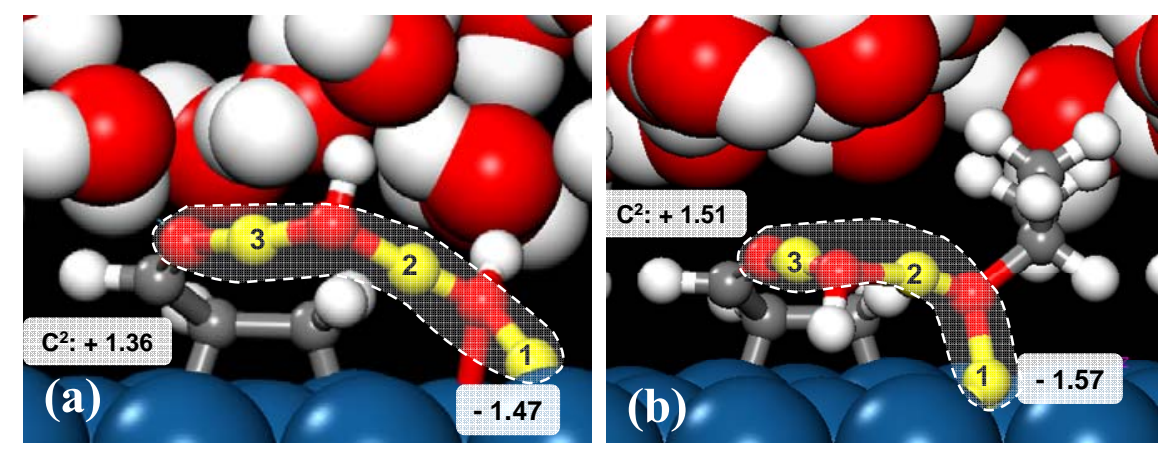

Figure 4.10 Charge analysis of the transition states of the proton shuttling mechanism of hydroxyl path in (a) water; (b) mixed IPA/water solvent with 10\% IPA over Pt(111). Hydrogen atoms involved in proton shuttling are shown in yellow.

The possibility of coupling acrolein hydrogenation with IPA dehydrogenation was also examined. Desai et al. previously reported barriers for methanol dehydrogenation of $92 \mathrm{~kJ} \mathrm{~mol}^{-1}$ for the $\mathrm{C}-\mathrm{H}$ activation and $142 \mathrm{~kJ}$ $\mathrm{mol}^{-1}$ for the $\mathrm{O}-\mathrm{H}$ activation over $\mathrm{Pt}(111)$ in vapor phase [111]. As summarized in Table 4.3, in the $10 \%$ mixed IPA-water solvent both the $\mathrm{O}-\mathrm{H}$ and $-\mathrm{H}$ bond activation of IPA show significantly higher activation barriers $\left(186 \mathrm{~kJ} \mathrm{~mol}^{-1}\right.$ and $124 \mathrm{~kJ} \mathrm{~mol}^{-1}$, respectively) than that from the hydrogen transfer reactions. At $375 \mathrm{~K}$ the rate for $\mathrm{C}-\mathrm{H}$ activation can be estimated to be $10^{-5} \mathrm{~s}^{-1}$ for $\mathrm{C}-\mathrm{H}$ bond activation (with pre-exponential factor of $10^{13}$ $\mathrm{s}^{-1}$ [87].) and only $10^{-13} \mathrm{~s}^{-1}$ for O-H bond activation. These rates are significantly lower than that for the rate of acrolein hydrogenation thus dehydrogenation hence could be considered as negligible in the mixed solvent over Pt.

Table 4.3 DFT predicted activation barriers $\left(\mathrm{kJ} \mathrm{mol}^{-1}\right)$ and reaction energies $\left(\mathrm{kJ} \mathrm{mol}^{-1}\right)$ of the IPA dehydrogenation in the $10 \%$ mixed IPA-water solvent.

\begin{tabular}{c|ccc}
\hline Pathways & $E_{a c t}$ & $\Delta E_{r x n}$ & $\Delta E_{\text {ads }}$ of product \\
\hline$\left(\mathrm{CH}_{3}\right)_{2} \mathrm{CH}-\mathrm{OH} \rightarrow\left(\mathrm{CH}_{3}\right)_{2} \mathrm{CHO}^{*}+\mathrm{H}^{*}$ & 186 & 135 & -80 \\
$\left(\mathrm{CH}_{3}\right)_{2} \mathrm{CH}-\mathrm{OH} \rightarrow\left(\mathrm{CH}_{3}\right)_{2} \mathrm{C}^{*} \mathrm{OH}+\mathrm{H}^{*}$ & 124 & 50 & -114 \\
\hline
\end{tabular}




\section{CONCLUSIONS}

First-principle theoretical simulations were used to show that protic solvents can lower the activation barriers and alter the controlling reaction mechanisms in the selective hydrogenation of unsaturated aldehydes on model transition metal surfaces. More specifically we showed the differences in the selective hydrogenation of acrolein over model $\operatorname{Pt}(111)$ surface when carried out in the gas phase, in pure water and in a $10 \%$ mixed isopropyl alcohol (IPA)-water solvent. Ab initio molecular dynamics and simulated annealing studies were carried out to determine reliable structures for the metal-solvent interface which are important in determining the adsorption and reactant on these surfaces.

In the absence of solvent, the surface reactions of acrolein favor the selective hydrogen addition at the $\mathrm{C}=\mathrm{O}$ bond via the hydroxyl hydrogenation path to yield unsaturated alcohol. The very slow desorption of unsaturated alcohol limit the observed selectivities. In the presence of solvent, acrolein binds at the solventmetal interface and forms hydrogen bonds with solvent molecules within its local solvation shell with the oxygen atom. Both water and the mixed solvent were found to enhance the hydrogenation of $\mathrm{C}=\mathrm{O}$ bond via hydroxyl route over that for the hydrogenation of the $\mathrm{C}=\mathrm{C}$ bond, The accessibility of the $\mathrm{O}-\mathrm{H}$ reaction center to the bulk solvent allows it to form hydrogen bonding with solvent molecules in the transition state which leads to extra stabilization over the reactant state and lowers the activation barriers by $10-20 \mathrm{~kJ} \mathrm{~mol}^{-1}$. The solvent-phase catalytic hydrogenation of the $\mathrm{C}=\mathrm{C}$ bond have barriers that are similar or somewhat higher than those found in the vapor phase, as the reaction involves the addition of hydrogen to the hydrophobic carbon centers. As such there is no hydrogen bonding or stabilization and as result the protic solvents are unable to promote these reactions.

A detailed charge analysis suggests that there is less polarization in the transition state in the hydroxyl route for mixed solvent system than that for the pure water. The charge distribution in the transition state stabilizes the metal-mediated path observed in mixed solvent $\left(22 \mathrm{~kJ} \mathrm{~mol}^{-1}\right)$ over that carried out in water $\left(30 \mathrm{~kJ} \mathrm{~mol}{ }^{-1}\right)$. Rates of $\mathrm{C}=\mathrm{C}$ bond hydrogenation at $375 \mathrm{~K}$ were estimated to be an order of magnitude smaller in the mixed solvent than those in water, which may enhance the probability of $\mathrm{C}=\mathrm{O}$ hydrogenation and rationalize the increased selectivity to unsaturated alcohol in the $10 \%$ mixed IPA-water solvent as compared to that in pure water.

Protic solvents can directly participate in the reaction mechanism by co-catalyzing proton transfer steps. The surface hydrogenation reactions can proceed via proton-coupled-electron transfer mechanisms where the surface hydride transfers its electron back into the surface into the adsorbed ketone or aldehyde thus resulting in the protonation of the key intermediate. The shuttling mechanism largely depends on the strength of the hydrogen bonds. In water the solvent-mediated hydroxyl route is expected to be dominant in the hydrogenation of acrolein, as the barrier $\left(21 \mathrm{~kJ} \mathrm{~mol}^{-1}\right)$ is $\sim 10 \mathrm{~kJ} \mathrm{~mol}^{-1}$ lower than that of metal-mediated route. In mixed 
solvent the solvent-mediated path $\left(35 \mathrm{~kJ} \mathrm{~mol}^{-1}\right)$ is not as favorable as the metal-mediate path $\left(22 \mathrm{~kJ} \mathrm{~mol}^{-1}\right)$ to form hydroxyl group, as the large alkyl group of IPA disrupts and weakens the facile proton transport network. The dehydrogenation of IPA was considered to be negligible in the mixed solvent over Pt due to the high activation barriers and thus extremely slow rates.

As presented here, solvents can significantly lower the activation barriers as compared to the vapor phase reaction by stabilizing the partial-charged transition state, and, in addition, altering the preferred reaction mechanism by directly participating in the reaction. The above correlates well with the increased selectivity to unsaturated alcohol observed in experiments in $10 \%$ mixed IPA-water solvent when compared to the pure solvents, and shed light to the possibility of tailoring the catalytic performance through controlling the structural dynamics (i.e., changing the composition) of the solvents. 


\subsection{Effects of Non-Polar Aromatic Solvents: Hydrogenation of Benzylacetone in Toluene}

Alkanes, aromatics and other hydrocarbon are often used as solvents for carrying out selective hydrogenation reactions as they provide an optimal media for solubilizing reagants and products, optimizing heat transfer and a variety of other important reasons. These solvents offer no stabilization due to hydrogen bonding and no way in which to shuttle protons. These solvents interact quite differently with the metal surface and there can be significant differences in comparing solvents such as $N$-alkanes (e.g., hexane) that weakly interact with metal surfaces with aromatics such as benzene, toluene or other substituted aromatics that can strongly bind to the surface.

Herein we examine the selective hydrogenation of benzylacetone over supported metal particles in an effort to complement our previous studies on benzalacetone reported in Chapter 3.1. Benzalacetone is an unsaturated ketone which predominantly reacted by the hydrogenation of the $\mathrm{C}=\mathrm{C}$ bond to form benzylacetone as the product which desorbed from the surface. Benzylacetone, however, can be hydrogenated if the reactions are carried out in hydrocarbon solvents. Benzylacetone is not an $\alpha, \beta$-unsaturated ketone but is comprised of phenyl and ketone groups that can compete for hydrogenation.

The liquid-phase hydrogenation of benzylacetone (see Figure 4.11) over supported Pt catalysts was recently examined in different solvents by our collaborators at Queen's University (Hardacre and Thompson groups) [54]. The rates of reaction were found to follow the following order: hexane $>$ cyclohexane $=$ IPA $>$ tert-butyl toluene $>$ toluene. As shown by Table 4.4 [54], hexane and IPA solvents lead to the fastest rates of reaction but favor the hydrogenation of the aromatic ring of benzylacetone, while toluene, an aromatic solvent, readily binds to the surface of most transition metal catalysts and demonstrates much slower rates of reaction but significantly favors the selectivity to the desired phenylbutanol product. The tert-butyl toluene solvent which is more substituted than toluene showed much higher rates for ring hydrogenation of benzylacetone than that of toluene solvent. The experimental results suggest that toluene may adsorb on the catalyst and block surface sites and thus inhibit the adsorption and ring hydrogenation of benzylacetone. The bulky side chain of tertbutyl toluene can considerably weaken the adsorption of t-butyl toluene and thus limit its ability to efficiently block surface sites. These are only, hypotheses for the moment concerning possible ways in which the solvent can increase the rates of reaction. The complexity of the solvent, metal and support interface presents a number of challenges that would make it difficult to establish the mechanisms by which the solvent influences the rate and selectivity for selective hydrogenation reactions via experiment alone. 


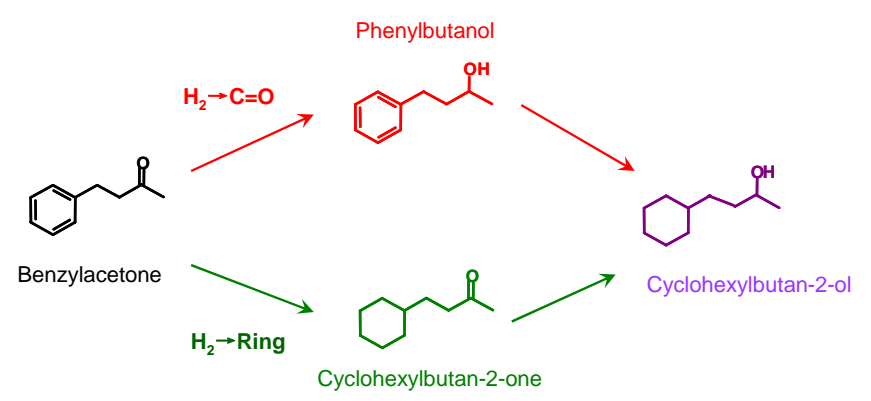

Figure 4.11 Competitive hydrogenation pathways and products of benzylacetone [54].

Table 4.4 Experimental selectivities (\%) observed in the liquid phase hydrogenations of benzylacetone over supported Pt catalysts in different solvents ${ }^{\mathrm{a}}$ [54].

\begin{tabular}{cccc}
\hline Solvent & Phenylbutanol (\%) & Cyclohexylbutan-2-one (\%) & Cyclohexylbutan-2-ol (\%) \\
\hline Hexane $^{\mathrm{b}}$ & 19 & 72 & 9 \\
$\begin{array}{c}\text { Isopropyl alcohol } \\
\text { (IPA) }\end{array}$ & 37 & 53 & 10 \\
Toluene $^{\mathrm{c}}$ & 82 & 17 & 1 \\
Tert butyl toluene $^{\mathrm{c}}$ & 62 & 30 & 8 \\
\hline
\end{tabular}

${ }^{\mathrm{a}}$ Reaction conditions: $100 \mathrm{mg} 4 \% \mathrm{Pt} / \mathrm{TiO}_{2}$ catalyst, $2 \mathrm{~g}$ benzylacetone, $50 \mathrm{~mL}$ solvent, 5 bar $\mathrm{H}_{2}, 70^{\circ} \mathrm{C}$. ${ }^{\mathrm{b}}$ Selectivity is reported at $\sim 45 \%$ conversion. ${ }^{\mathrm{c}}$ Selectivity is reported at $\sim 5 \%$ conversion.

We have used first principle theoretical studies [68] herein to probe the influence of non-polar aromatic solvents, toluene and tert-butyl toluene, on the hydrogenation of benzyl acetone over the model $\mathrm{Pt}(111)$ surface. The influence of the toluene solvent on the adsorption and reactivity of benzylacetone as well as the mechanisms that control the selective hydrogenation were examined in detail. In addition, we also explored the adsorption and selective hydrogenation of the toluene solvent to form methylcyclohexane as this is an unselective and competing reaction that can also proceed.

\section{Computational Details}

The Pt(111) surface was modeled using a $4 \times 4$ unit cell with the bulk lattice constants for Pt is a=3.99 $\AA$. The structures of the reactants, intermediates and products were optimized until the forces on all of atoms were less than $0.05 \mathrm{eV} / \AA$ using a three-step procedure. The first and second steps were carried out to provide a good estimate of the structures using a conservative $3 \times 3 \times 1$ Monkhorst-Pack k-point mesh [71] to sample the Brillouin zone [72] with an electronic convergence criterion of $1 \times 10^{-6} \mathrm{eV}$. The final step is carried out at the highest level of accuracy by using a $6 \times 6 \times 1$ k-point mesh using a static single-point calculation. Negative 
adsorption energies indicate that the adsorption is exothermic and favorable. A two step approach which combines the nudged elastic band (NEB) method [74-77] along with the dimer method [78-80] is used to isolate reaction transition states. In NEB calculation, a series of 8 images are taken at fixed points along the reaction path. These images are optimized using a $3 \times 3 \times 1 \mathrm{k}$-point until the maximum force on each atom decreased to $0.20 \mathrm{eV} / \AA$. The dimer calculations were then carried out by $3 \times 3 \times 1 \mathrm{k}$-point until the maximum atomic forces converged to $0.05 \mathrm{eV} / \AA$, and finally a $6 \times 6 \times 1 \mathrm{k}$-point was used to determine the energy of the transition state (TS) in a static single-point calculation.

\section{Results and Discussions}

The hydrogenation of benzlacetone proceeds by the adsorption of hydrogen and benzlacetone followed by the sequential addition of hydrogen to the $\mathrm{C}$ and $\mathrm{O}$ atoms of the carbonyl as well as to the carbon atoms of in the benzyl group. The modes and energies of adsorption of benzylacetone on $\operatorname{Pt}(111)$ were examined over a range of different toluene coverages. The symbol " $N$ " was used to indicate the number of toluene molecules per unit cell. As demonstrated by Figure 4.12, benzylacetone adsorbs favorably with its aromatic ring in a planar to the clean $\operatorname{Pt}(111)$ surface $(N=0)$ via strong $\pi-\pi$ interactions with a adsorption energy of $-55 \mathrm{~kJ} \mathrm{~mol}^{-1}$. The adsorption energy of benzylacetone significantly decreases with increasing coverage of toluene as a result of lateral repulsive interactions. Toluene also binds through its aromatic ring via $\pi-\pi$ interactions and has very similar adsorption energies as that of benzylacetone (Figure 4.12). As such, toluene and benzylacetone competitively adsorb on the surface.

Previous surface science experiments carried out at ultra-high vacuum conditions [112] indicate that aromatic molecules such as cinnamaldehyde bind in a parallel orientation, onto an ideal single crystal metal surface up to a surface density of $2.7 \times 10^{14}$ molecules per $\mathrm{cm}^{2}$. The saturation coverage for aromatics in our system is then calculated to be 2 molecules per $4 \times 4$ unit cell of $\operatorname{Pt}(111)$. As the binding energies for toluene and benylacetone are similar and the concentrations of toluene solvent dominate over benzylacetone, we would expect toluene to be the dominant species on the surface with $N \geq 1$. Benzylacetone can also adsorb but it does so solely through the interaction with the $\mathrm{C}=\mathrm{O}$ groups at vacancy sites on the toluene-covered surfaces. While the interaction through $\mathrm{C}=\mathrm{O}$ group is significantly weaker $\left(-17 \mathrm{~kJ} \mathrm{~mol}^{-1}\right.$ on the clean $\mathrm{Pt}$ surface), it is essentially independent of the toluene coverage. As shown in Figure 4.12, the adsorption energies of benzylacetone via its $\mathrm{C}=\mathrm{O}$ bond were calculated to be $16 \mathrm{~kJ} \mathrm{~mol}^{-1}$ and $13 \mathrm{~kJ} \mathrm{~mol}^{-1}$ at toluene coverage of $N=1$ and $N=2$, respectively, which are very similar to the case in absence of toluene. 


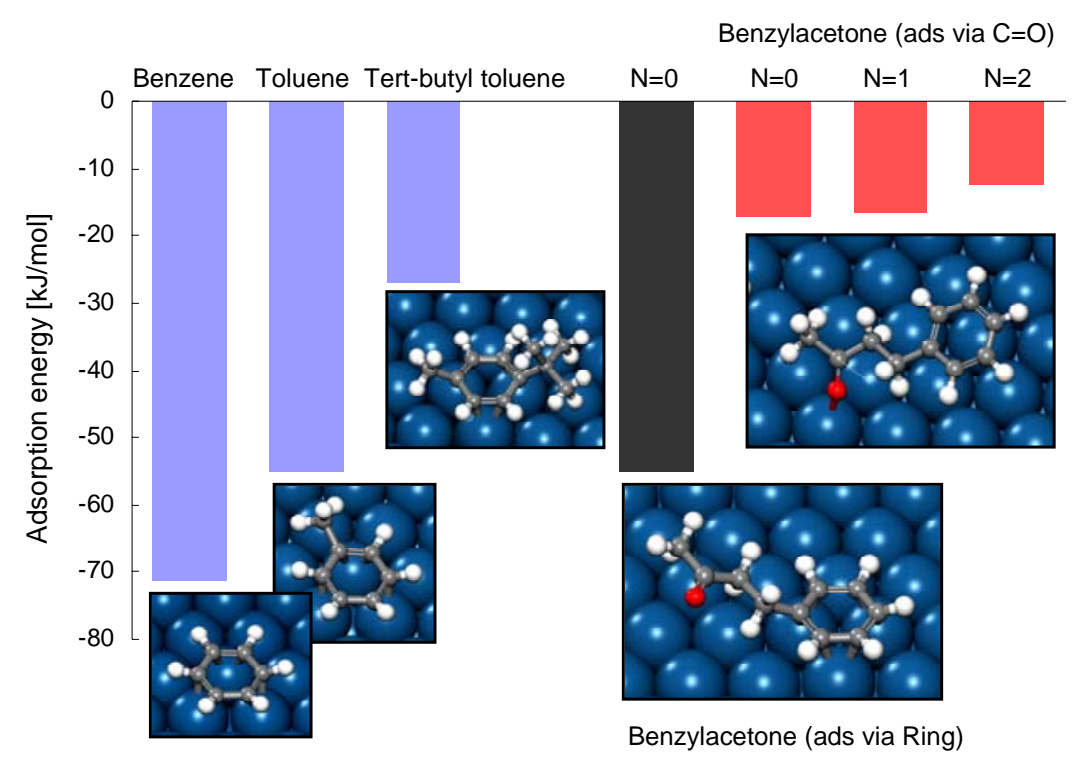

Figure 4.12 Adsorption energies $\left(\mathrm{kJ} \mathrm{mol}^{-1}\right)$ of toluene, tert-butyl toluene and benzylacetone on $\operatorname{Pt}(111)$ surface in absence of $(N=0)$ and presence of toluene $(N=1, N=2)$. " $N$ " indicated the number of toluene molecules per unit cell.

The competitive paths in the hydrogenation of benzylacetone on the clean $\operatorname{Pt}(111)$ surface are shown in Figure 4.13. Surface hydrogen can either attack the $\mathrm{C}=\mathrm{O}$ bond to form phenylbutanol or add to the aromatic ring to form cyclohexylbutan-2-one. On the clean Pt surface $(N=0)$, the hydrogenation of benzylacetone appears to predominantly occur at the aromatic ring sites, due to its strong binding of benzylacetone to the surface (Figure 4.14). While the intrinsic barriers to hydrogenate the $\mathrm{C}=\mathrm{O}$ bond ( 71 and $77 \mathrm{~kJ} \mathrm{~mol}^{-1}$ for the hydroxyl and alkoxide path, respectively) are slightly lower than those to hydrogenate the aromatic ring (78 94 $\mathrm{kJ} \mathrm{mol}^{-1}$ ), the apparent barriers to hydrogenate the $\mathrm{C}=\mathrm{O}$ bond are much higher due to the weaker adsorption of benzylacetone in the $\eta^{2}(\mathrm{C}=\mathrm{O})$ configuration $\left(-17 \mathrm{~kJ} \mathrm{~mol}^{-1}\right)$ for $\mathrm{C}=\mathrm{O}$ hydrogenation as compared to the parallel $\eta^{6} \pi$-bound configuration $\left(-55 \mathrm{~kJ} \mathrm{~mol}^{-1}\right.$ ) for the ring hydrogenation. This can be seen by examining the total energies reported for the TSp5 $\left(-59 \mathrm{~kJ} \mathrm{~mol}^{-1}\right)$ and TSp1 $\left(-28 \mathrm{~kJ} \mathrm{~mol}^{-1}\right)$ states shown in Figure 4.14, as referenced to benzylacetone and $\mathrm{H}_{2}$ in gas phase $(E=0)$. Of the different solvents examined experimentally and reported in Table 4.4, hexane provides the closest match to the vapor phase results reported here as it is relatively non-interacting solvent which only weakly interacts with the metal. The DFT results reported here which indicate that benzylacetone predominantly hydrogenates the aromatic ring over the $\mathrm{C}=\mathrm{O}$ group are consistent with the experimental results for the reactions carried out in hexane reported in Table 4.4 [54]. 


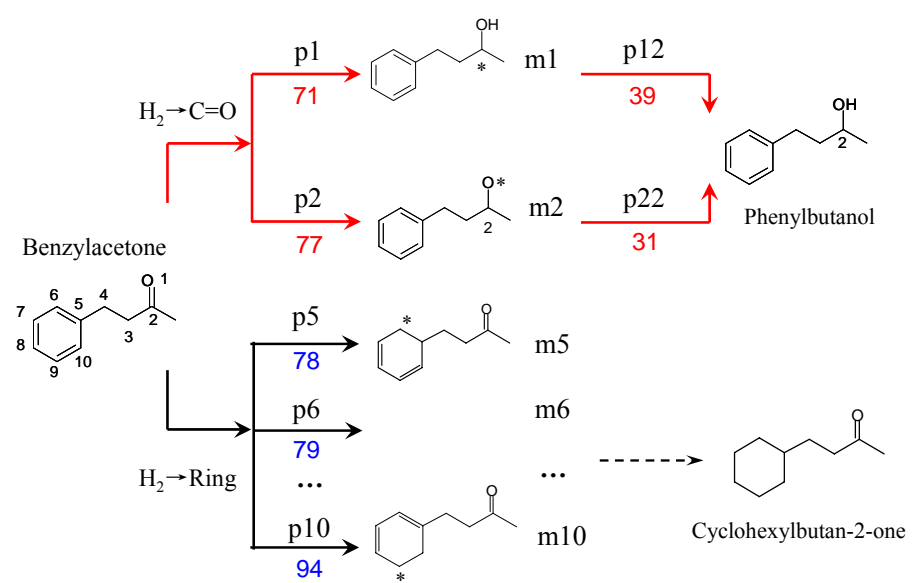

Figure 4.13 Pathways for hydrogenation of benzylacetone on the clean $\operatorname{Pt}(111)$ surface. The first hydrogenation intermediates $\mathrm{m} 1, \mathrm{~m} 2$, and $\mathrm{m} 5-\mathrm{m} 10$ are named after the sites (centers) of hydrogen attack. Pathways p1-p2 indicate the elementary reactions of the first hydrogenation step and p12-p22 refer to the second hydrogenation reactions of the $\mathrm{C}=\mathrm{O}$ bond. Pathways $\mathrm{p} 5$-p10 refer to the first hydrogenation reactions of the aromatic ring. Activation barriers are reported in $\mathrm{kJ} \mathrm{mol}^{-1}$. The '*' refers to an unsaturated carbon or oxygen atom after the initial hydrogenation step.

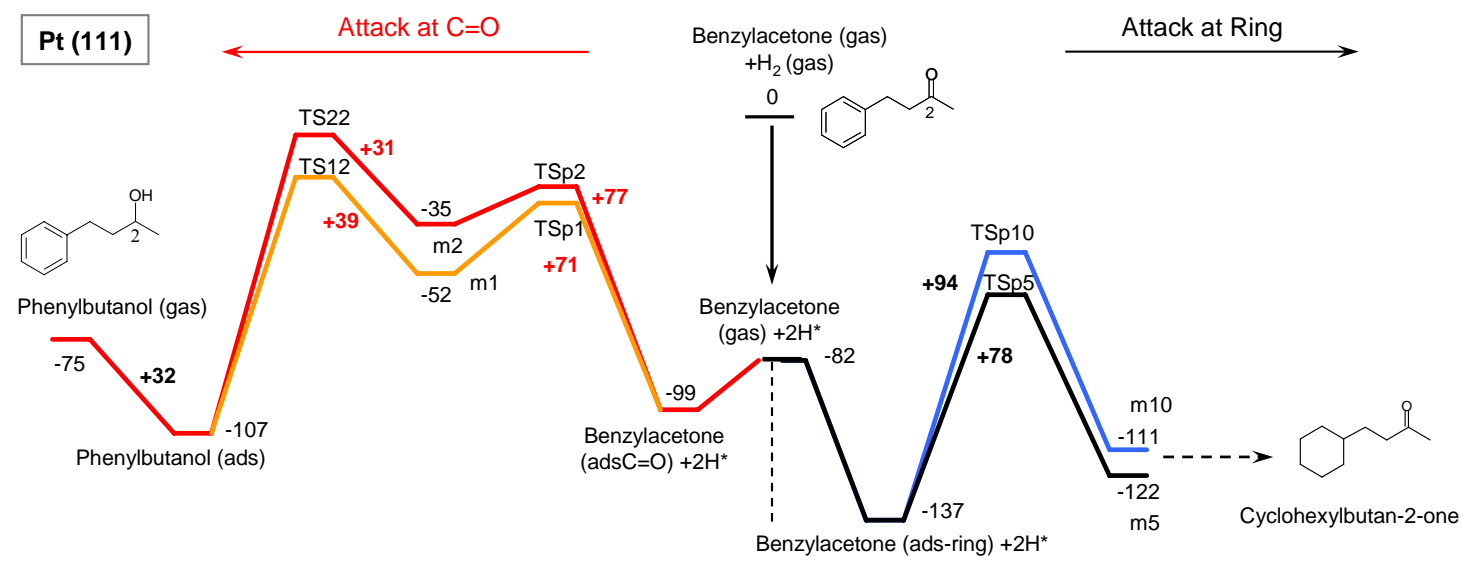

Figure 4.14 DFT-calculated potential energy profiles of the partial hydrogenation of benzylacetone over clean $\mathrm{Pt}(111)$ surface. Reaction energies and activation barriers are in $\mathrm{kJ} \mathrm{mol}^{-1}$.

The activation barriers and the transition states for the ring hydrogenation of benzylacetone and toluene over clean $\operatorname{Pt}(111)$ surface are compared in Figure 4.15 and 4.16. The activation barriers for hydrogenating the aromatic ring in toluene (76-92 $\mathrm{kJ} \mathrm{mol}^{-1}$ ) are very close to those for hydrogenating benzylacetone (78-94 $\mathrm{kJ}$ $\mathrm{mol}^{-1}$ ). Experimental results indicate that if this reaction is carried out in hexane with addition of toluene, only a small amount of the toluene (3\%) is converted to methylcyclohexane. This suggests that toluene is blocking the sites responsible for hydrogenating the aromatic ring of benzylacetone rather than competing with reactant for hydrogen [54]. 


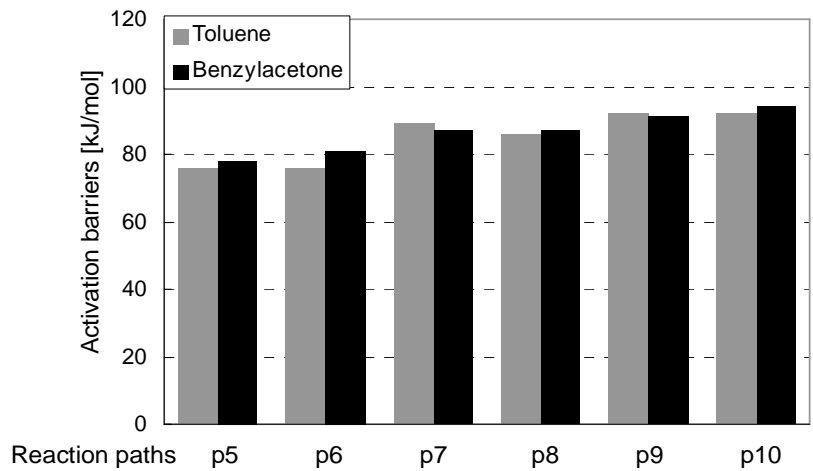

Figure 4.15 Activation barriers for the elementary paths in hydrogen addition to the aromatic ring of benzylacetone and toluene on clean $\mathrm{Pt}(111)$ surface. Energies are reported in $\mathrm{kJ} \mathrm{mol}^{-1}$.
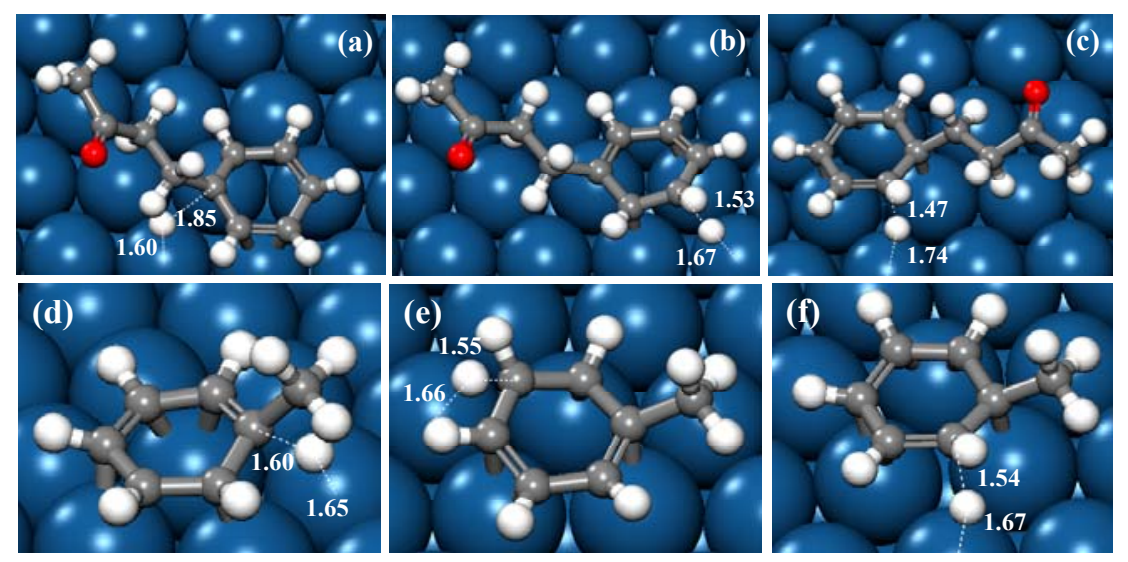

Figure 4.16 Transition states configurations for the ring hydrogenation of benzylacetone (a) path p5; (b) path p7; (c) path p10; and of toluene (d) path p5; (e) path p7; (f) path p10 over clean Pt (111) surface. Bond lengths are in $\AA$.

In the presence of toluene $(N \geq 1)$, the Pt surface is covered by toluene. The low coverage $(N=0)$ clean $\mathrm{Pt}$ surface model that we have used so far is no longer adequate for simulating the surface chemistry. As the surface is thought to be covered in toluene, we use instead the toluene-covered ( $N=1$ and $N=2$ models) surfaces to model the elementary steps in the hydrogenation of the benzylacetone.

As Pt surface is covered in toluene, benzylacetone can only adsorb onto the surface at exposed Pt sites that lie between adsorbed toluene intermediates in a vertical configuration via its $\mathrm{C}=\mathrm{O}$ group as is shown in Figure 4.12. This is consistent with experimental measurements which show that the rate of hydrogenation is extremely slow as most sites on the Pt sites are blocked by toluene [54]. The selectivity to the phenylbutanol in toluene, however, increases significantly from $19 \%$ for the reactions carried out in hexane to $82 \%$ for the reactions carried out in toluene (Table 4.4). The benzyl group of benzylacetone cannot bind via their benzyl 
rings to the toluene-covered surface and can only interact and react via the $\mathrm{C}=\mathrm{O}$ group which binds in an $\eta^{2}$ configuration where the benzyl group is oriented away from the surface. Table 4.5 summarizes the activation barriers for the $\mathrm{C}=\mathrm{O}$ bond hydrogenation of benzylacetone at different coverages of toluene on $\mathrm{Pt}(111)$. The presence of toluene significantly increases the barriers to hydrogenate the oxygen center of the carbonyl ( $\sim 30 \mathrm{~kJ} \mathrm{~mol}^{-1}$ higher than that in absence of toluene), while the barriers to form the alkoxide intermediate are barely affected by the toluene concentration on Pt. The alkoxide route is therefore considered to be favorable for the initial hydrogenation reaction of the carbonyl group of benzylacetone in presence of toluene.

The same reactions were also carried out in the tertiary-butyl substituted toluene solvent. The results experimental results reported in Table 4.4 indicate that the reaction is less selective to the phenolbutanol when carried out in tert-butyl toluene instead of toluene. Tert-butyl substituted toluene is more sterically hindered than toluene and as a result it binds more weakly to Pt $\left(-27 \mathrm{~kJ} \mathrm{~mol}^{-1}\right)$ than toluene does $\left(-55 \mathrm{~kJ} \mathrm{~mol}^{-1}\right)$ and therefore does not block the surface sites to the same extent as toluene does and as such should lead to higher rates of reaction but slightly lower selectivity to phenolbutanol as benzylacetone reactants can now react to form cyclohexylbutan-2-one from the ring hydrogenation.

Table 4.5 Activation barriers $\left(E_{a c t}, \mathrm{~kJ} \mathrm{~mol}^{-1}\right)$ and reaction energies $\left(E_{r x n}, \mathrm{~kJ} \mathrm{~mol}^{-1}\right)$ for the $\mathrm{C}=\mathrm{O}$ bond hydrogenation of benzylacetone on $\operatorname{Pt}(111)$ surface in absence of $(N=0)$ and presence of toluene $(N=1, N=2)$.

\begin{tabular}{ccccc}
\hline \multicolumn{2}{c}{ Coverage of Toluene } & $N=0$ & $N=1$ & $N=2$ \\
\hline \multirow{2}{*}{ Hydroxyl path p1 } & $E_{a c t}$ & 71 & 96 & 98 \\
& $E_{r x n}$ & 47 & 73 & 76 \\
\multirow{2}{*}{ Alkoxide path p2 } & $E_{a c t}$ & 77 & 80 & 82 \\
& $E_{r x n}$ & 64 & 61 & 66 \\
\hline
\end{tabular}
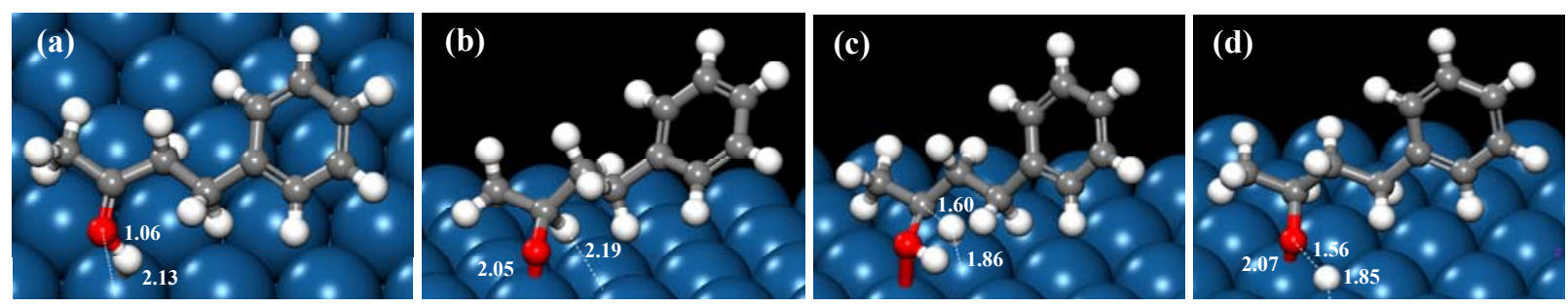

Figure 4.17 Transition states configurations of the $\mathrm{C}=\mathrm{O}$ bond hydrogenation for (a) path $\mathrm{p} 1$; (b) path p2; (c) path p12; (d) path p22 of benzylacetone over clean Pt (111) surface. Bond lengths are in $\AA$. 


\section{Conclusions}

Non-polar solvents cannot form hydrogen bonds or hydrogen bonding networks and as such do not offer the same stabilization of polar transition states or proton shuttling pathways that protic solvents do but can still markedly affect the reactivity and selectivity by altering the surface property of the catalyst. Aromatic solvents such as toluene and other aromatics tend to adsorb strongly onto the transition metal surfaces and can block surface sites. We specifically explored the influence of toluene and tert-butyl toluene on the adsorption and hydrogenation of benzylacetone over model $\mathrm{Pt}(111)$ surfaces and compared with known experimental results. In absence of toluene, benzylacetone adsorbs favorably with its aromatic ring in a planar to the $\mathrm{Pt}(111)$ surface via $\pi$ - $\pi$ stacking interactions and predominantly undergoes hydrogenation of the benzyl ring to form cyclohexylbutan-2-one due to the strong interactions of benzylacetone and the surface. The results are consistent with the experimental results for reactions carried out in the non-interacting hexane solvent. The adsorption energies for toluene, a more strongly interacting solvent, as well as the intrinsic activation barriers to hydrogenate toluene were found to be very close to those of benzylacetone. This suggests that toluene and benzylacetone competitively adsorb on the Pt surface. Experiments carried out in hexane with only small amounts of toluene (3\%) converted to methyl-cylcohexane suggest that toluene competitively blocks sites for benzylacetone but does not compete with reactant for hydrogen.

In presence of toluene, the Pt surface is covered by toluene and that benzylacetone can only adsorb via its $\mathrm{C}=\mathrm{O}$ group in a vertical mode at exposed Pt sites between adsorbed toluene surface intermediates. The binding through $\mathrm{C}=\mathrm{O}$ bond is rather weak $\left(-17 \mathrm{~kJ} \mathrm{~mol}^{-1}\right)$ and nearly independent to the coverage of toluene. The rate of hydrogenation of $\mathrm{C}=\mathrm{O}$ bond of benzylacetone is thus rather slow as most surface sites are blocked by toluene. The selectivity to the phenylbutanol, however, is significantly higher than that for reactions carried out in other solvents such as hexane and isopropanol as the only available path for hydrogenation on the toluene-covered surface is via the $\mathrm{C}=\mathrm{O}$ group. The alkoxide pathway was not significantly influenced by the toluene concentration and hence was favorable for the initial hydrogenation of carbonyl in presence of toluene. The bulky side chain of tert-butyl toluene limits its ability to efficiently block the surface sites and therefore lead to a higher rate of reaction. The weaker interactions of tert-butyl toluene with Pt allows for the competitive adsorption of benzylacetone in its $\pi$-bound $\eta^{6}$ configuration and a somewhat lower selectivity to phenylbutanol $(62 \%)$ as result of the increased formation of the cyclohexylbutan-2-one (30\%). This work, in collaboration with experimental studies, can help to elucidate the role of aromatic solvents in altering the surface properties and facilitating the formation of desired alcohol products, and in addition help to establish relationships between the solid-liquid interaction and catalytic activity/selectivity. 


\section{Chapter 5 -Manganese Oxide Octahedral Molecular Sieves (OMS-2) and Pt/OMS-2 as Novel Catalysts for the Hydrogenation of Unsaturated Aldehydes}

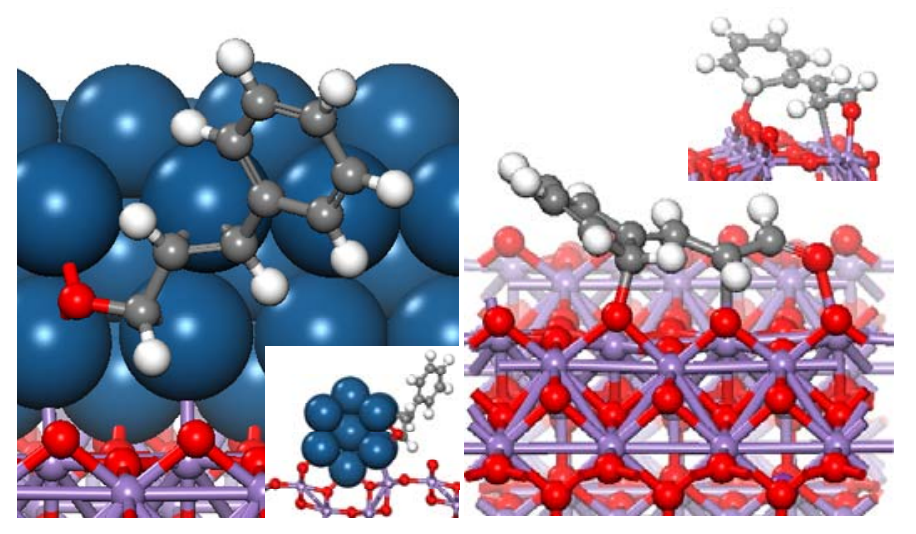

unsaturated oxygenates may be tailored by an appropriate choice of OMS-2 alone or OMS-2 supported metal catalysts.

Manganese oxide octahedral molecular sieves (OMS-2) were found to be a highly selective catalysts for $\mathrm{C}=\mathrm{C}$ bond hydrogenation to form saturated aldehydes, whereas $\mathrm{Pt}$ supported on OMS-2 favor $\mathrm{C}=\mathrm{O}$ over $\mathrm{C}=\mathrm{C}$ hydrogenation and result in high selectivity to the unsaturated alcohol. OMS-2 is thought to promote Pt and may directly participate in the catalytic reaction. The selectivity during the hydrogenation of ( choice or OMS-2 alone or OMS-2 supported

\section{INTRODUCTION}

The catalyst support can dictate the size, shape, stability and reactivity of the metal particles that carry out catalytic reactions. The support can also directly participate in carrying out different catalytic reactions by carrying out or aiding specific reactions. Support effects are thought to play an important role in the hydrogenation of unsaturated aldehydes and ketones [12, 13, 16, 18-23]. The nature and composition of the support can significantly alter the performance of supported metal catalysts $[18,22]$. The reaction selectivity to unsaturated alcohol of benzalacetone hydrogenation over $\mathrm{Au}$ was considerably enhanced when the reaction was carried out over $\mathrm{Fe}_{2} \mathrm{O}_{3}$ versus $\mathrm{TiO}_{2}$ or carbon $[13,16]$. Traditional supports such as oxides and activated carbons have been extensively examined and used in the hydrogenations of different unsaturated ketones and aldehydes. For example, $\mathrm{Ru} / \mathrm{C}$ and $\mathrm{Ru} / \mathrm{SiO}_{2}$ catalysts show much higher selectivities ( $20 \%$ or more) to the unsaturated alcohol than for $\mathrm{Ru} / \mathrm{Al}_{2} \mathrm{O}_{3}$ for cinnamaldehyde hydrogenation $[18,22]$. Unfortunately, most of these catalysts result in negligible or very low activities for the selective hydrogenation of the $\mathrm{C}=\mathrm{O}$ bond in unsaturated ketones $[12,13]$.

Recent efforts suggest that the octahedral molecular sieves of manganese oxides, and more specifically OMS-2 $[55,56]$, are active in carrying out hydrogenation reactions. OMS-2 are cryptomelane-type manganese oxides 
with porous structures $(0.46 \mathrm{~nm})$ arising from the edge and corner shared $\mathrm{MnO}_{6}$ octahedral units, forming $2 \times 2$ tunnel structures (Figure 5.1a-c). The chemical composition of cryptomelane-type OMS is $\mathrm{KMn}_{8} \mathrm{O}_{16} \cdot \mathrm{nH}_{2} \mathrm{O}$ with the $\mathrm{K}^{+}$cation inside the tunnel for charge balancing $[55,56]$. The framework consists of Mn species with the mixed valances of $+2,+3$, and +4 yielding an average oxidation state of +3.8 [55]. The multivalent character, along with the $2 \times 2$ tunnel structure with lattice oxygen vacancies, makes OMS- 2 a potential redox catalyst for selective oxidation (of alcohols [57, 58]), oxidative dehydrogenation (of ethane [55]), hydroxylation (of acetophenone [59]), hydrogenation (of nitroanisole [59]) and photocatalyst (of 2-propanol [113]).
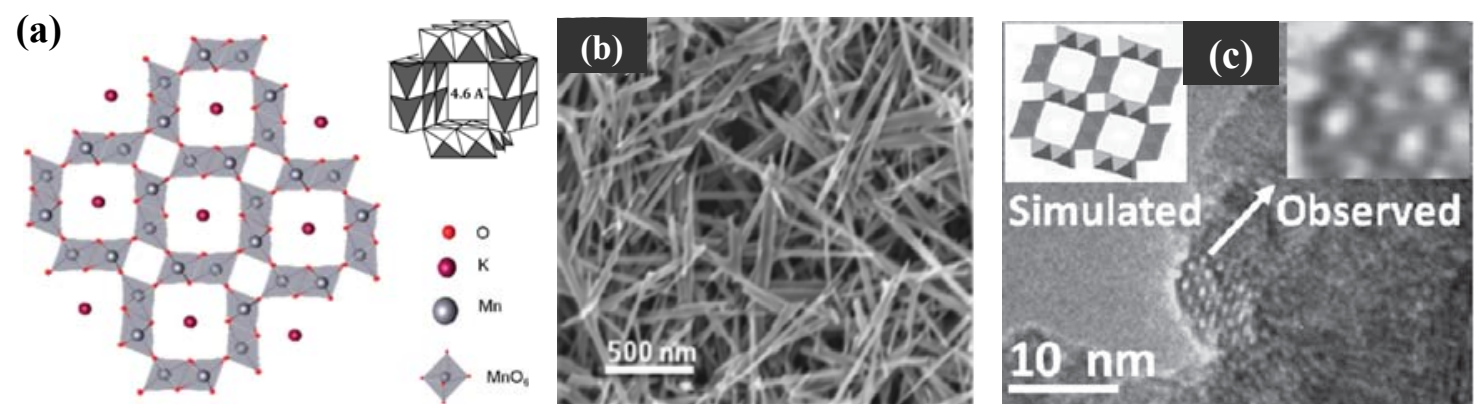

Figure 5.1 (a) Tunnel structures of cryptomelane type OMS-2. Potassium atoms reside in the tunnels are shown in brown, $\mathrm{MnO}_{6}$ octahedra are shown in ash, oxygen is in red color, and ash solid spheres are manganese [55]. (b) Fibrous morphologies of OMS-2 materials [55]. (c) Tunnels of the simulated and observed cryptomelane by HRTEM [56]. Reprinted with permission from Ref. [55], copyright 2012 from American Chemical Society. Reprinted with permission from Ref. [56], copyright 2011 from John Wiley \& Sons, Inc.

OMS-2 can also act as support to anchor metal particles such as Pt that can readily split hydrogen thus resulting in bifunctional sites that may act to improve catalytic performance for selective hydrogenation reactions. Recent studies by our collaborators at Queen's University in Belfast demonstrated that OMS-2 and Pt/OMS-2 could be used as catalysts for selective hydrogenation of $\alpha, \beta$-unsaturated aldehydes and ketones into unsaturated alcohols. They showed that these materials demonstrated unexpected selectivities for a number of reactions including the selective hydrogenation of ketoisophorone and cinnamaldehyde [60]. Experimental results suggest that OMS-2 alone can selectively hydrogenate the $\mathrm{C}=\mathrm{C}$ bonds to produce saturated ketone or aldehyde, whereas the Pt particles supported on OMS-2 more selectively facilitate the hydrogenation of the $\mathrm{C}=\mathrm{O}$ bonds to give unsaturated alcohol (Figure 5.2). The selectivities for the hydrogenating unsaturated ketones and aldehydes to unsaturated alcohols over Pt supported on conventional oxides such as $\mathrm{Al}_{2} \mathrm{O}_{3}$ are typically rather low[18]. The results for the selective hydrogenation of cinnamaldehyde to cinnamyl alcohol, for example, are summarized in Table 5.1. The activated carbon or carbon nanofiber supports were found to enhance the selectivity of UA up to $40-45 \%[13,114]$. The novel Pt/OMS-2 catalyst significantly enhanced the selective hydrogenation of the $\mathrm{C}=\mathrm{O}$ bond to form cinnamyl alcohol at selectivity of $80 \%$ at $96 \%$ conversion 
[60]. A more detailed understanding on the effects of the metal and metal particle size and the influence of the metal/support interface would provide important insights into the development of structure-activity relationships that may be used to further enhance the activity and selectivity of these materials. First principle density functional theory calculations [61] are used herein to help establish the role of the OMS-2 support, the $\mathrm{Pt}$ particle and Pt/OMS-2 interface in selectively hydrogenating unsaturated aldehydes and ketones.

Table 5.1 Experimental selectivities at given conversions for cinnamaldehyde hydrogenation over supported $\mathrm{Pt}, \mathrm{Ru}$ catalysts and the OMS-2 materials. UA: unsaturated alcohol (cinnamyl alcohol); SAL: saturated aldehyde (hydrocinnamaldehyde).

\begin{tabular}{ccccc}
\hline Catalyst & UA (\%) & SAL (\%) & Conversion (\%) & Ref. \\
\hline $\mathrm{Ru} / \mathrm{Al}_{2} \mathrm{O}_{3}$ & $13-30$ & - & 40 & {$[18]$} \\
$\mathrm{Ru} / \mathrm{C}$ & 41 & 39 & 61 & {$[13]$} \\
$\mathrm{Pt} / \mathrm{C}$ & 44 & 50 & 54 & {$[13]$} \\
$\mathrm{Ru} /$ carbon nanofiber & 48 & - & 60 & {$[114]$} \\
OMS-2 & - & 86 & 97 & {$[60]$} \\
$\mathrm{Pt} / \mathrm{OMS}-2$ & 80 & $<10$ & 96 & {$[60]$} \\
\hline
\end{tabular}

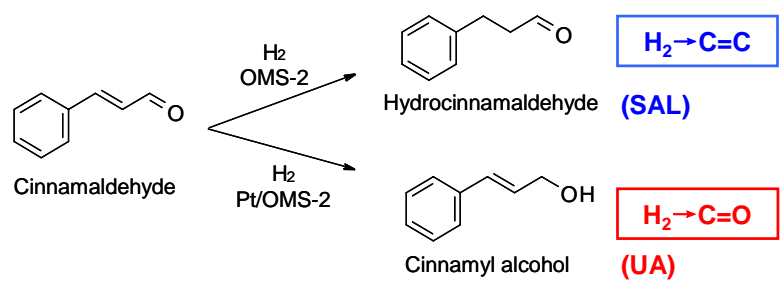

Figure 5.2 Competitive hydrogenation pathways and products of cinnamaldehyde on $5 \mathrm{wt} \% \mathrm{Pt} / \mathrm{OMS}-2$ and OMS-2 catalysts (adapted from [60]). Reprinted with permission from Ref. [60], copyright 2013 from John Wiley \& Sons, Inc.

Recently experimental efforts have used Raman scattering spectroscopy, X-ray diffraction and X-ray photoelectron spectroscopy to help determine the structure and properties of OMS-2 [115-117]. In addition, recent theoretical DFT $+U$ studies reported the atomic and magnetic structure of cryptomelane-type $\alpha-\mathrm{MnO}_{2}$ [118], which provides a benchmark for further computational studies of the OMS-2 materials as the supports for heterogeneous catalytic systems.

Previously we combined theoretical and experimental studies to elucidate the mechanisms and kinetics that control the hydrogenation of benzalacetone and cinnamaldehyde over supported Pt and Ru catalysts [13]. The results clearly show that the adsorption mode and binding strength of the surface species are important in controlling the hydrogenation activity as well as the selectivity in these reactions. Herein we extend our initial 
theoretical efforts and examine the elementary steps and mechanisms that govern the hydrogenation of cinnamaldehyde over OMS-2 and model Pt/OMS-2 materials. More specifically, we will examine the surface structures and compositions of the OMS-2 and Pt nanorods supported on OMS-2 to help elucidate the influence of the support on the electronic and structural properties of metal and their subsequent influence on the catalytic activity and selectivity. We examine the adsorption of the cinnemaldhyde, hydrogen and their reactivity at the Pt/OMS-2 interface and attempt to compare with recent experimental results.

\section{MODEL SYSTEM AND METHODOLOGY}

The structures of manganese oxide octahedral molecular sieves (OMS-2) with the $\mathrm{K}^{+}$inside the tunnels used in this work were constructed according to the theoretical structures reported for cryptomelane $\alpha-\mathrm{MnO}_{2}$ by Cockayne et al. [118]. The optimized crystallographic structure of OMS-2 is shown in Figure 5.3. In order to model the metal/support interactions we employed a Pt nanorod as it captures the nature of the metal/support interfacial sites and provides a stable framework for the metal. The model Pt nanorod used in this work used to mimic Pt particles supported on OMS-2 is 3 atomic layers and 3-atoms-wide and covalently bonded to the cryptomelane OMS-2 (001) surface. A $15 \AA$ vacuum gap was used to separate periodic slabs in z-direction. The bottom two Mn-O layers of the OMS-2 support were held fixed at their bulk-phase lattice positions, while the atoms in the top Mn-O layers were allowed to fully relax in the structure optimizations. All of the Pt atoms were allowed to relax in z-direction.

All of the calculations reported herein were carried out using periodic density functional theory (DFT) [61] as implemented in the Vienna ab-initio simulation package (VASP) [68]. Generalized gradient approximation (GGA) [69] was used to model the non-local gradient corrections to the correlation and exchange energies along with the recently-developed PBEsol (Perdew-Burke-Ernzerhof revised for solids[119]) parameterization. The interactions between the valence electrons and the core were described using the projector augmented wave method (PAW) [120, 121]. The wave functions were constructed from an expansion of plane waves with an energy cutoff of $400 \mathrm{eV}$.

The effects of magnetism of $\mathrm{Mn}^{4+}$ ion in the electronic structure studies of OMS-2 [118] were included via the DFT $+U(+J)$ approach to address the on-site strong Coulomb repulsion in the localized $d$ - or $f$ - orbitals of the magnetic $3 d$ oxides (Mn, Co). The energy functional of DFT $+U$ can be expressed as [122]:

$$
E^{D F T+U}=E^{D F T}+\frac{\bar{U}-\bar{J}}{2} \sum_{\sigma}\left[\operatorname{Tr} \rho^{\sigma}-\operatorname{Tr}\left(\rho^{\sigma} \rho^{\sigma}\right)\right]
$$

The effective Mn on-site Coulomb $(U)$ and the exchange $(J)$ values for Mn $(d)$ were set to $U=2.0 \mathrm{eV}, J=1.0$ $\mathrm{eV}$; for $\mathrm{O}(p), U=2.1 \mathrm{eV}, J=0.0 \mathrm{eV}[118]$. The magnetic state of Mn was set according to theoretical findings 
by Cockayne et al. [118] who showed that the $\mathrm{Mn}-\mathrm{Mn}$ coupling between corner-sharing $\mathrm{MnO}_{6}$ is antiferromagnetic and interactions between edge-sharing $\mathrm{MnO}_{6}$ in the neighboring columns are weakly ferromagnetic. The initial magnetic moment for $\mathrm{Mn}$ and $\mathrm{O}$ elements $( \pm 4 \mu \mathrm{B}$ for $\mathrm{Mn}, 0 \mu \mathrm{B}$ for $\mathrm{O}$ ) were set according to the experimental magnetic moments in the vacancy-free $\mathrm{MnO}_{2}(-0.04 \mu \mathrm{B}$ for $\mathrm{O}$ and $3.08 \mu \mathrm{B}$ for $\mathrm{Mn})$ [123].

Geometric structures were optimized until the force on each atom was less than $0.01 \mathrm{eV} / \AA$. A $2 \times 6 \times 2$ Monkhorst-Pack k-point mesh [71] was used to optimize the structural geometry of OMS-2, and a $3 \times 3 \times 1$ Monkhorst-Pack mesh was used to examine the interactions of hydrogen with the model Pt/OMS-2 structure. The nudged elastic band (NEB) method [74-77] was combined with the Dimer method [78-80] to isolate transition states. In the NEB calculations, an initial series of 8 intermediate images were refined using a $3 \times 3 \times 1$ k-point mesh until the maximum force on each atom decreased to $0.20 \mathrm{eV} / \AA$. The dimer was subsequently constructed by replicating the structure of the system with very small change in distance between each state (set to $0.01 \AA$ ), and rotated through a minimization of the torque to ensure that the dimer is in-line with the MEP. The dimer calculations were carried out by $3 \times 3 \times 1 \mathrm{k}$-point until the maximum atomic forces converged to $0.05 \mathrm{eV} / \AA$, and finally a $6 \times 6 \times 1 \mathrm{k}$-point was used to determine the energy of the transition state (TS) in a static single-point calculation. The charge density difference $\left(\mathrm{CHG}_{\mathrm{diff}}\right)$ on the OMS-2 surface before and after Pt deposition was calculated using a Bader charge analysis [74-77], where $\mathrm{CHG}_{\mathrm{diff}}=\mathrm{CHG}(\mathrm{Pt} / \mathrm{OMS}-2)-$ CHG(OMS-2) - CHG(Pt)

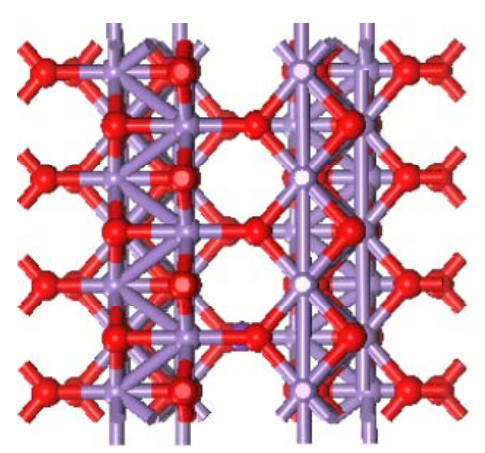

(a)

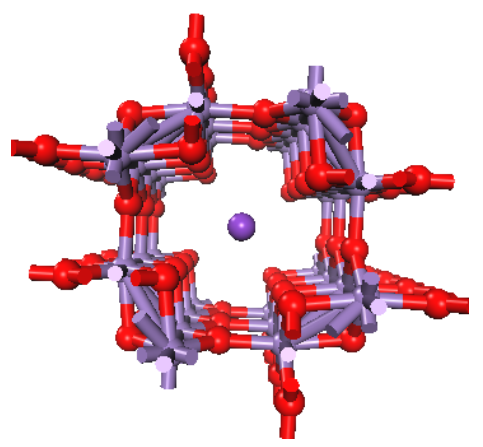

(b)

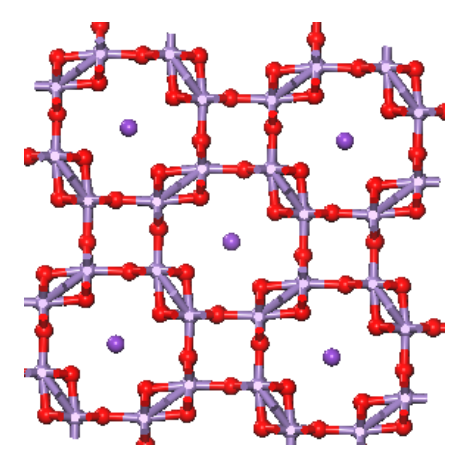

(c)

Figure 5.3 Crystal structures of manganese oxide octahedral molecular sieves (OMS-2) with the $\mathrm{K}^{+}$located inside the tunnel. (a) Top view; (b) perspective view; (c) crystal frameworks. Purple: Mn; red: O; dark purple inside the tunnel: $\mathrm{K}^{+}$. 


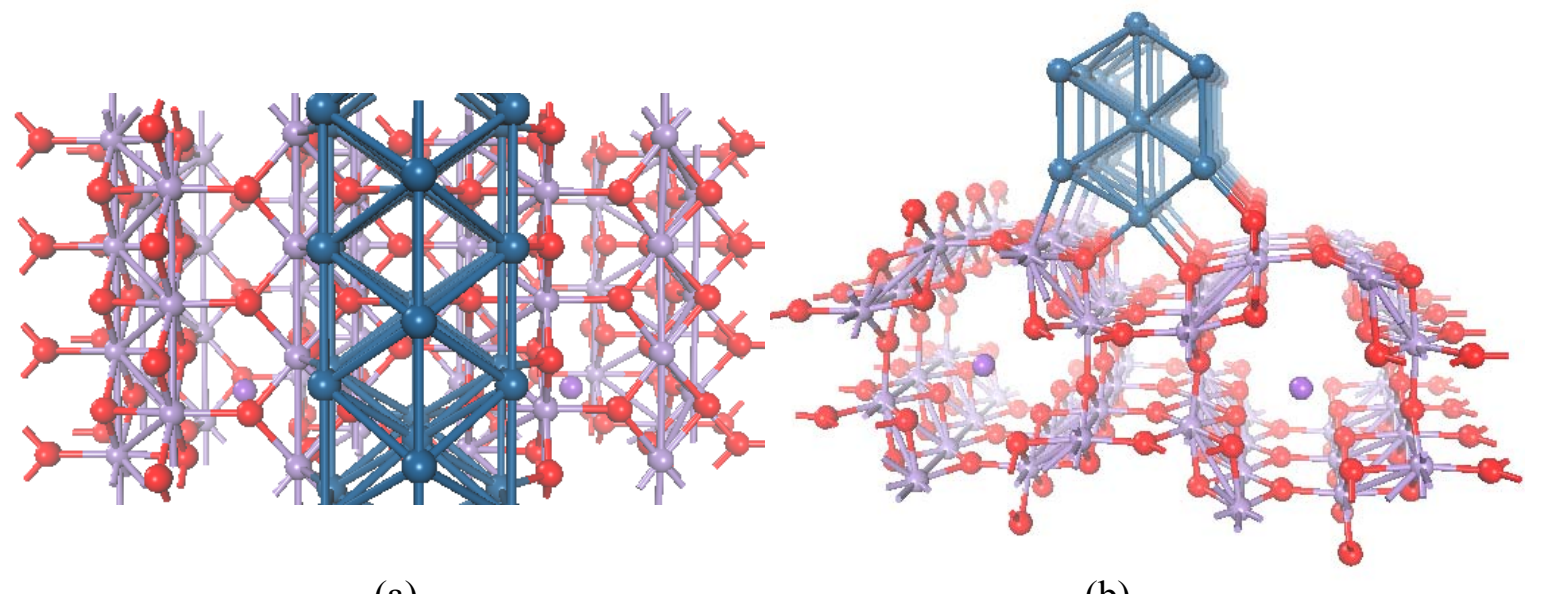

(a)

(b)

Figure 5.4 (a) Top view and (b) perspective view of the 3-atomic-layer Pt nanorod supported by the cryptomelane OMS-2 (001) surface model. Pt atoms are in blue.

\section{RESULTS AND DISCUSSION}

\section{1) Atomic and Electronic Structures of OMS-2 and Pt/OMS-2 Catalysts}

The DFT $+U$ optimized crystallographic structures of OMS- 2 with the $\mathrm{K}^{+}$inside the tunnel are displayed in Figure 5.3. The optimized Mn-O bond length was calculated to be $\sim 1.90 \AA$. The lowest-energy position of cation $\mathrm{K}^{+}$shows distances between $\mathrm{K}^{+}$and closest $\mathrm{Mn}$ and $\mathrm{O}$ as $3.56 \AA$ and $2.85 \AA$, which correlates well with previous experimental and theoretical studies of K-OMS-2 material [115, 118]. Atomic charge calculation indicated that the addition of $\mathrm{K}^{+}$to OMS-2 reduces one $\mathrm{Mn}^{4+}$ to $\mathrm{Mn}^{3+}$, which agrees with the electronic structure of $\alpha-\mathrm{MnO}_{2}$ [118]. The DFT $+U$ optimized unit cell parameters for OMS-2 $(a=9.702 \AA, b=2.856 \AA, c=$ $9.685 \AA)$ were also in good agreement with experimental diffraction patterns ( $a=c=9.815 \AA$ and $b=2.847 \AA[56$, $118])$.

To model the Pt/OMS-2 catalysts used experimentally, we use a model Pt nanorod 3 atomic layers high and 3 atomic layers wide supported on the cryptomelane OMS-2 (001) surface as shown in Figure 5.4. The Pt nanorod structure was chosen because it provides a reliable and stable model of the terrace and edge sites at the Pt/OMS-2 interface. The electronic charge difference $\left(\mathrm{CHG}_{\text {diff }}\right)$ at the Pt/OMS interface before and after Pt deposition is plotted in Figure 5.5. The adsorption of Pt rod on OMS-2 leads to a significant transfer of electrons from the Pt to the OMS-2 support. As indicated by the red regions (higher electronic density) in the dotted rectangles in Figure 5.5, negative charge accumulates on the $\mathrm{O}$ and $\mathrm{Mn}$ sites at the perimeter of $\mathrm{Pt}$ rod, as the Pt atoms that are direct bound to the OMS-2 support donate electrons to the support and become 
positively charged. This change in charge density is responsible for the strong adsorption of hydrogen over the OMS-2 support (Table 4.2).

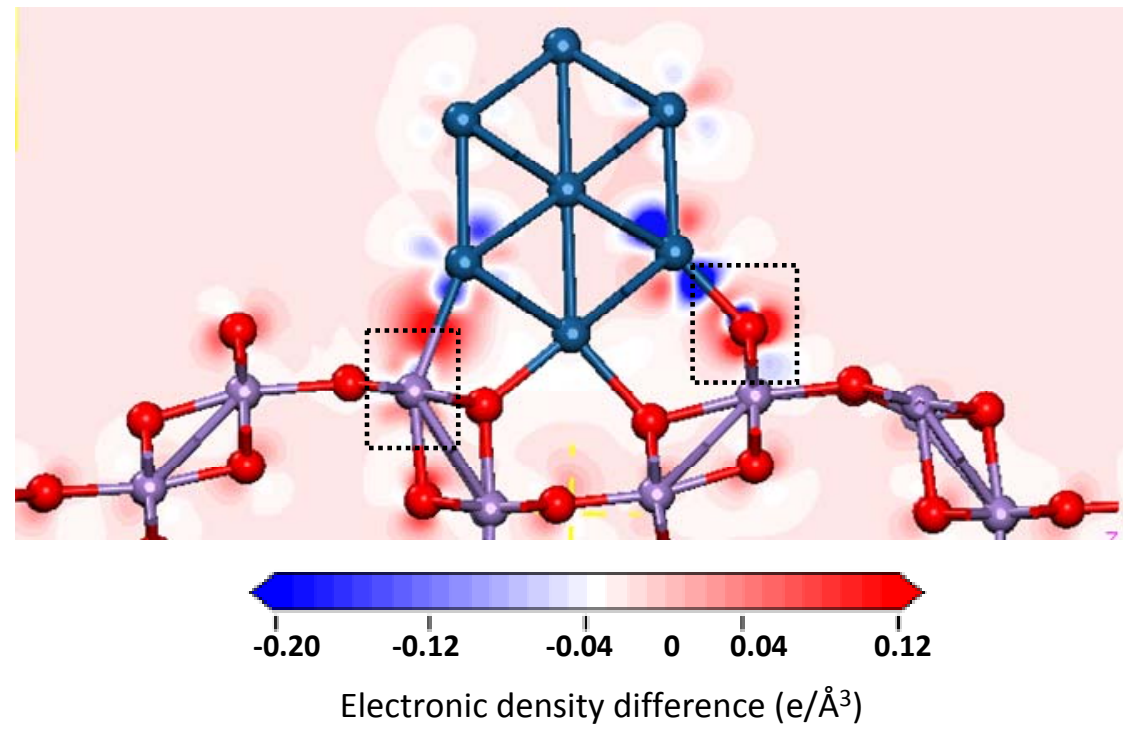

Figure 5.5 Charge density differences before and after Pt deposition on the OMS-2 surface. The figure shows the side view of the symmetric 3-atomic-layer Pt nanorod supported by OMS-2 surface. Red regions exhibit increasing electronic density, and blue regions exhibit decreasing electronic density.

\section{2) Models of the Active Sites}

The reactions at the Pt/OMS-2 interface can proceed in various ways which might lead to the promotional effects found experimentally. We begin by first exploring the dissociative adsorption of hydrogen which can proceed via 1) heterolytic activation on OMS-2, 2) homolytic activation on $\mathrm{Pt}$, or 3) heterolytic activation at bifunctional Pt-Mn sites at the metal-support interface. There is also the possibility that $\mathrm{H}_{2}$ is activated on $\mathrm{Pt}$ and spills over onto the support.

Similarly, cinnamaldehyde reactant can adsorb onto the supported Pt rod or onto the OMS-2 support and the hydrogenation can then proceed solely at the interfacial Pt sites or the OMS-2 sites. Cinnamaldehyde may also adsorb at the bifunctional Pt-Mn sites at the interface to create an interfacial Pt- $(\mathrm{R}) \mathrm{C}=\mathrm{O}-\mathrm{Mn}\left(\delta^{+}\right)$during the hydrogenation reaction. The latter mechanism is very similar to the proposed models for $\mathrm{O}_{2}$ adsorption and activation on $\mathrm{Au} / \mathrm{TiO}_{2}$ interface [124]. We will then explore the hydrogen activation, cinnamaldehyde adsorption and the following hydrogenation at the model active sites which are outlined above. 


\section{3) Hydrogen Activation}

Hydrogen can be activated either over the Pt, on the OMS-2 support or at the interface. In addition, $\mathrm{H}_{2}$ can be activated on Pt and spill over onto the OMS-2 supports [125-127]). Recent literature suggests that reverse spillover of hydrogen (from the receptor to the source where they are recombined) can occur when a metal is added to the support and was used to explain the increasing rate of alcohol dehydrogenation [128]. The behavior of hydrogen on OMS-2 and the metal doped OMS-2 is critical for understanding the mechanisms and the active sites that carry out hydrogenation over the OMS-2 based catalysts.

The possible binding sites of atomic hydrogen on the OMS-2 supported Pt symmetric nanorod, the OMS-2 surface without Pt and the stand-alone Pt nanorod are shown in Figure 5.6 in cross section views and labeled by number (1)-(13) for an easy identification. The calculated adsorption energies for atomic hydrogen at these sites are summarized in Table 5.2.

The binding of atomic $\mathrm{H}$ to the OMS-2 support (referred to site (1)-(7)) was found to be very strong, especially at the bridging oxygen sites $\left(-400 \mathrm{~kJ} \mathrm{~mol}^{-1}\right.$ at site (1), see Table 5.2) which are rather basic. It is therefore difficult for $\mathrm{H}$ bound to these sites to migrate or add to the bound cinnamaldehyde regardless of whether it is on the metal or on the support. The barriers for migration and hydrogenation from these sites are rather high [127]. The adsorption of hydrogen on OMS-2 doped with Pt was found to be $\sim 30 \mathrm{~kJ} \mathrm{~mol}^{-1}$ stronger than that on the bare OMS-2 support (Table 5.2). When Pt is brought into contact with OMS-2 support, there is a charge transfer from the Pt atoms to the oxygen sites on the support thus making them more basic and increasing their affinity for hydrogen.

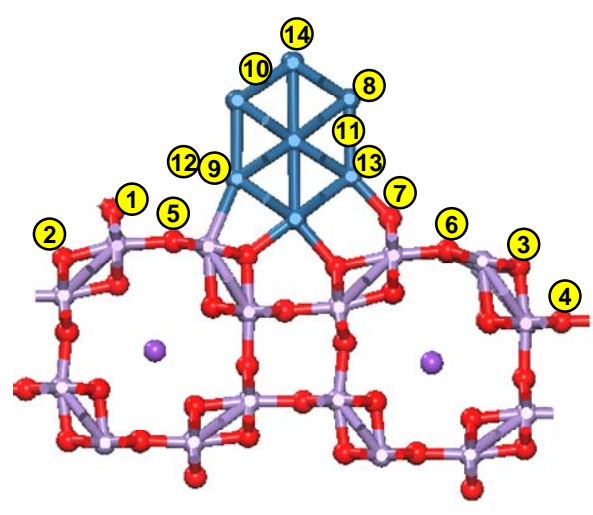

(a)

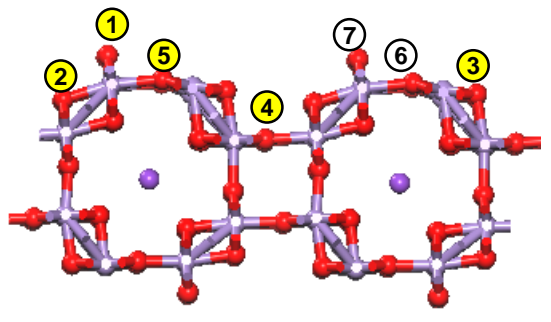

(b)
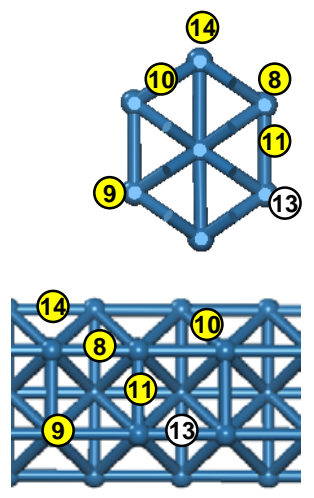

(c)

Figure 5.6 Possible hydrogen binding sites at the: (a) Pt/OMS-2 interface; (b) bare OMS-2 (001) surface; (c) Pt nanorod. 
Table 5.2 Binding energies $\left(\mathrm{kJ} \mathrm{mol}^{-1}\right)$ of atomic hydrogen at the Pt/OMS-2 interface, bare OMS-2 (001) surface and the stand-alone Pt nanorod. The energies reported are referred to the atomic hydrogen in gas phase. Hydrogen binding sites are labeled as (1)-(13) according to Figure 5.6.

\begin{tabular}{|c|c|c|c|c|}
\hline & Site & Supported Pt/OMS-2 & $\begin{array}{c}\text { Bare OMS-2 (001) } \\
\text { surface }\end{array}$ & Pt rod \\
\hline \multirow{7}{*}{$\begin{array}{c}\text { OMS } \\
\text { support } \\
\text { (oxygen) }\end{array}$} & 1 (bridge oxygen) & -400 & -370 & - \\
\hline & 2 (corner) & -322 & -293 & - \\
\hline & 3 (corner) & -309 & -285 & - \\
\hline & 4 (valley) & -300 & -264 & - \\
\hline & 5 (terrace) & -277 & -251 & - \\
\hline & 6 (terrace) & -223 & equal to (5) & - \\
\hline & 7 (O-Pt bond) & -241 & equal to (1) & - \\
\hline \multirow{7}{*}{ Pt rod } & 8 (bridge_111) & -289 & - & -296 \\
\hline & 9 (atop_100) & -244 & - & -251 \\
\hline & 10 (bridge_111) & -253 & - & -267 \\
\hline & 11 (bridge_100) & -249 & - & -275 \\
\hline & 12 (bridge_100_Mn) & -274 & - & equal to (8) \\
\hline & 13 (bridge_100_O) & -236 & - & equal to (8) \\
\hline & 14 (bridge_111) & -276 & - & -276 \\
\hline
\end{tabular}

The binding of hydrogen to the Pt sites at the Pt/OMS-2 interface was found to be about $10 \mathrm{~kJ} / \mathrm{mol}$ weaker than on the Pt nanorod alone (Table 5.2). The weaker interaction of $\mathrm{H}$ on Pt near the support is due to the electron transfer that occurs from the metal to the support. The weakest $\mathrm{H}$ binding energies $\left(\sim 240 \mathrm{~kJ} \mathrm{~mol}^{-1}\right)$ are observed at the Pt sites (9) and (13) which are directly attached to the OMS-2 support. The most stable binding site on a Pt rod is at the undercoordinated Pt-Pt bridge site (8) at (111) facet $\left(-289 \mathrm{~kJ} \mathrm{~mol}^{-1}\right)$. The 3 -fold site binding energy of a hydrogen atom on $\operatorname{Pt}(111)$ is $-262 \mathrm{~kJ} \mathrm{~mol}^{-1}(-2.72 \mathrm{eV})$, for comparison.

The hydrogen that is formed on the Pt sites can diffuse to the interface and sequential spillover onto the OMS2 support. We examine two possible pathways as illustrated in Figure 5.7a. The energies for these paths are given in Figure 5.7b. Hydrogen species can readily migrate on the surface of the Pt nanorod and can react with cinnamaldehyde that is also adsorbed on the Pt rod. The barriers for hydrogen diffusion on Pt from site (13) to (8) is only $6 \mathrm{~kJ} \mathrm{~mol}^{-1}$ and $19 \mathrm{~kJ} \mathrm{~mol}^{-1}$ from site (8) to (12). In contrast, the barriers for hydrogen diffusion on the OMS-2 support are significantly higher as the hydrogen binds much more strongly to the oxygen sites on the support. The diffusion of the hydrogen bound to the bridge $\mathrm{O}(1)$ site, which is the strongest binding site, to the $\mathrm{O}(5)$ terrace site is very difficult with a barrier of $164 \mathrm{~kJ} \mathrm{~mol}^{-1}$. The diffusion of hydrogen on the bare OMS-2 surface without $\mathrm{Pt}$ is easier with barriers that are $15-20 \mathrm{~kJ} \mathrm{~mol}^{-1}$ lower. The results suggest that the 
active hydrogen are likely localized at the Pt sites and perimeter oxygen sites and do not readily diffuse across the OMS-2 support at low temperatures. This is consistent with speculations from the experimental results which suggest that the rates of hydrogenation on OMS-2 are limited by the slow dissociation and diffusion of hydrogen as the initial rates are highly dependent upon the hydrogen pressure but only change slightly with the reactant concentration [60]. The great enhancement in rate ( $\sim 6$ fold in experiments) after doping OMS-2 with $\mathrm{Pt}[60]$ can also be attributed to the more facile diffusion of hydrogen on Pt to the interfacial reaction sites and the greater concentrations of hydrogen at these sites. This would suggest that hydrogenation is more likely to occur at the perimeter Pt sites and the interfacial Pt/OMS-2 sites.

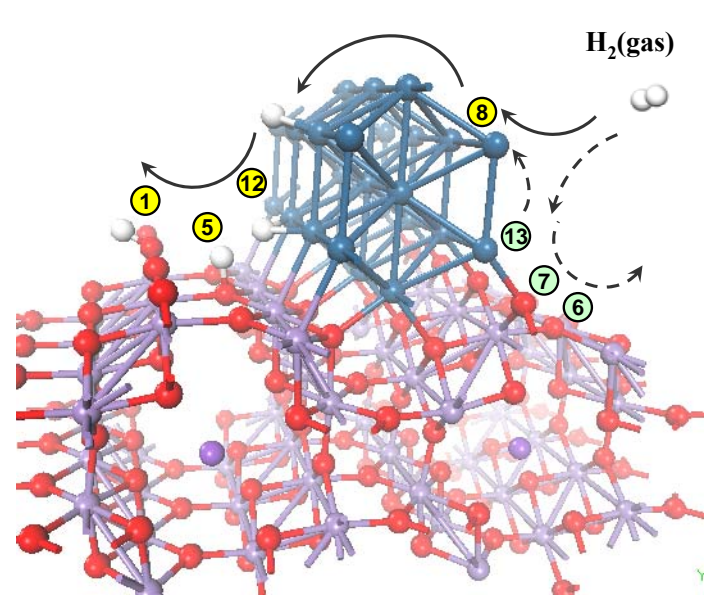

(a)

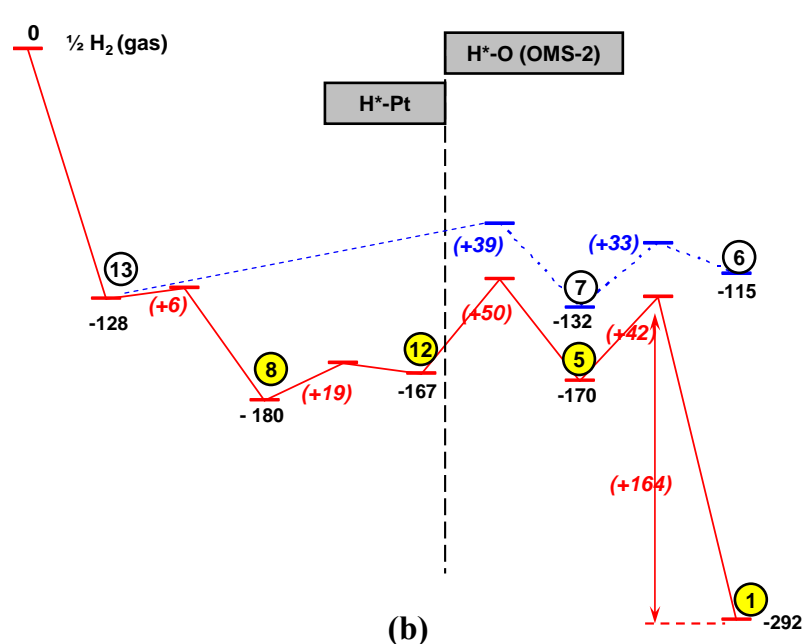

(b)

Figure 5.7 (a) Spillover of atomic hydrogen from the supported Pt rod to the OMS-2 support along two postulated pathways. (b) The corresponding potential energy scheme with diffusion barriers and energy differences in $\mathrm{kJ} \mathrm{mol}^{-1}$. The numbers in parenthesis are diffusion barriers.

\section{4) Cinnamaldehyde Adsorption and Hydrogenation over Bare OMS-2}

Cinnamaldehyde can be selectively hydrogenated to either cinnamyl alcohol (UA), a key building block in pharmaceutics, or hydrocinnamaldehyde (SAL), which is widely used in perfumery as a cinnamon floral odor [60], by the hydrogenation of the $\mathrm{C}=\mathrm{O}$ bond or $\mathrm{C}=\mathrm{C}$ bond, respectively. The adsorption sites, configurations and their energies for the reactant, products and intermediates (m1-m4, as depicted in Figure 5.8) were calculated on the bulk Pt(111) surface ( $4 \times 4$ unit cell), the bare OMS-2 (001) surface, and the supported Pt/OMS-2 and are compared in Table 5.3, Figure 5.9 and Figure 5.10. The adsorptions of these intermediates were examined only at the top surface of the slab. 
The adsorption of cinnamaldehyde on bare OMS-2 (001) surface was found to be very weak with a binding energy of only $-12 \mathrm{~kJ} \mathrm{~mol}^{-1}$ (Figure 5.9c). As depicted by Table 5.4, hydrogenation on the bare OMS-2 surface has significantly high activation barriers $\left(>100 \mathrm{~kJ} \mathrm{~mol}^{-1}\right)$, which are $30 \sim 100 \mathrm{~kJ} \mathrm{~mol}^{-1}$ higher than those on $\mathrm{Pt}(111)$ surface or on the supported Pt nanorod. This is the result of the very strong binding of hydrogen to the oxygen sites of the OMS-2 surface which require significant energy penalties in order to migrate and add to the bound reactant, as is shown in the transition states reported in Figure 5.12 (a-2 to d-2). The reaction rate on OMS-2 should therefore be much lower than that on Pt, as is observed experimentally [60].

Hydrogenation at the $\mathrm{C}=\mathrm{C}$ double bond is highly favored on the bare OMS-2 surface, as the activation barriers (113 and $122 \mathrm{~kJ} \mathrm{~mol}^{-1}$ for path $\mathrm{c} 1$ and $\mathrm{d} 1$, respectively) are much lower than those to hydrogenate the carbonyl group (136 and $156 \mathrm{~kJ} \mathrm{~mol}^{-1}$ for path a1, b1) for both the first and the second hydrogen addition steps. The OMS-2 mediated hydrogenation is also likely to be limited by the slow diffusion of hydrogen on the OMS surface and, as such, the products that form are likely to be those more thermodynamically stable in gas phase. Both the slow diffusion and the formation of the more stable gas phase intermediates are consistent with the results and suggestions from experiments [60]. In gas phase the saturated aldehyde (hydrocinnamaldehyde, $\mathrm{SAL}$ ) from $\mathrm{C}=\mathrm{C}$ hydrogenation is calculated to be $\sim 20 \mathrm{~kJ} \mathrm{~mol}^{-1}$ more stable than the cinnamyl alcohol (UA). These agree with the experimental observation that the reaction using OMS-2 itself as catalyst went to the hydrogenation of $\mathrm{C}=\mathrm{C}$ bond to produce $\mathrm{SAL}$ with $86 \%$ selectivity at conversion of $97 \%$ [60].

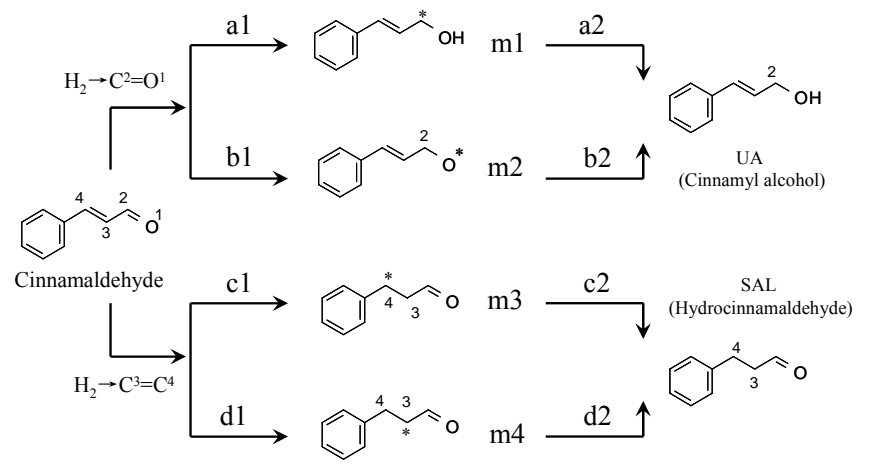

Figure 5.8 Four competitive hydrogenation routes of cinnamaldehyde. The intermediates from the first hydrogen addition, $\mathrm{m} 1, \mathrm{~m} 2, \mathrm{~m} 3$ and $\mathrm{m} 4$, are numbered according to the center that the hydrogen adds to. Paths a1, b1, $\mathrm{c} 1, \mathrm{~d} 1$ refer to the reactions for the first hydrogen addition,, and a2, b2, c2, d2 refer to the reactions for the second hydrogen addition . '*' refers to an unsaturated carbon or oxygen site that is bound to the metal surface after the initial hydrogen addition. 
(a)

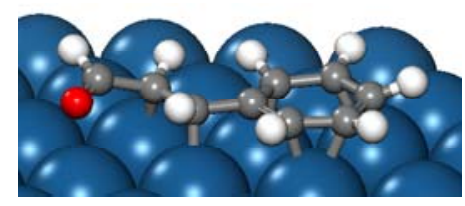

(b)
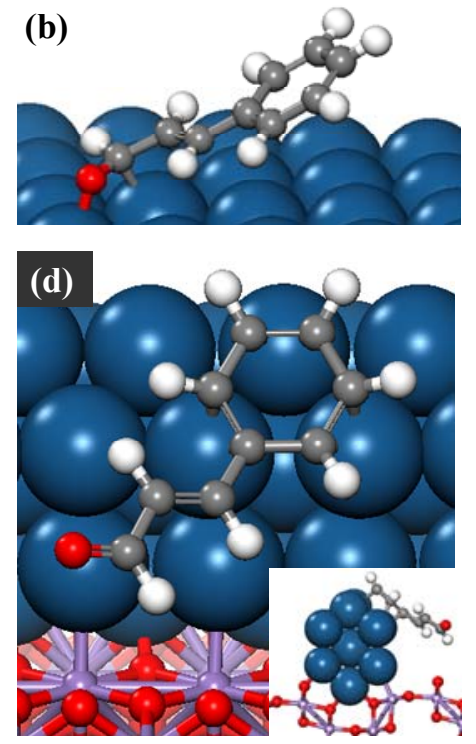

(c)
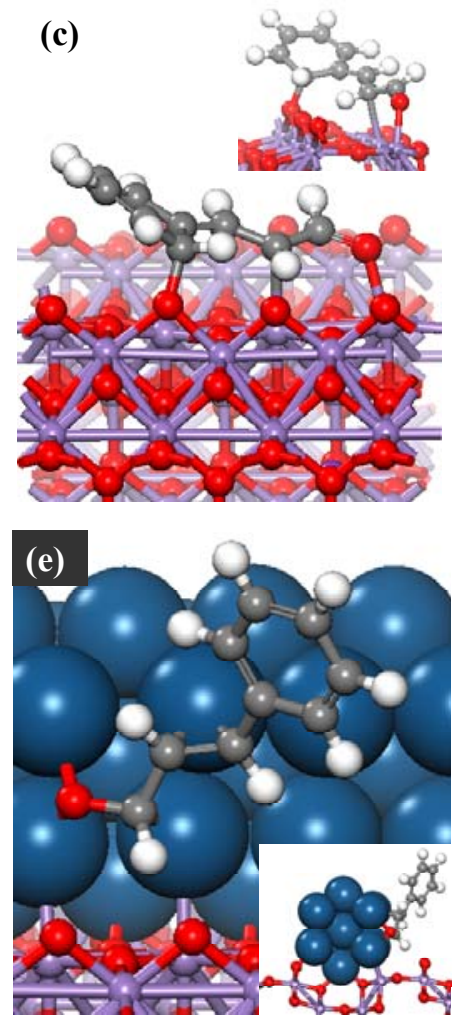

Figure 5.9 Adsorption geometries of cinnamaldehyde on: (a) $\mathrm{Pt}(111)$ surface via the aromatic ring and $\mathrm{C}=\mathrm{C}$ bond; (b) $\mathrm{Pt}(111)$, only via the $\mathrm{C}=\mathrm{O}$ bond; (c) bare OMS-2 (001) surface; (d) on the Pt nanorod supported by OMS-2 via the aromatic ring; and (e) on the $\mathrm{Pt}$ rod supported by OMS-2, via both the $\mathrm{C}=\mathrm{C}$ and $\mathrm{C}=\mathrm{O}$ bond.

Table 5.3 Adsorption energies $\left(\mathrm{kJ} \mathrm{mol}^{-1}\right)$ for the reactants, intermediates and products of cinnamaldehyde hydrogenation on the bulk Pt(111), bare OMS-2 surface, and the Pt nanorod supported by OMS-2.

\begin{tabular}{cccc}
\hline Specie & $\begin{array}{c}\text { Bulk } \\
\text { Pt(111) }\end{array}$ & $\begin{array}{c}\text { Bare OMS-2 (001) } \\
\text { surface }\end{array}$ & $\begin{array}{c}\text { Supported } \\
\text { Pt/OMS-2 }\end{array}$ \\
\hline Cinnamaldehyde & $-98^{\mathrm{a}},-21^{\mathrm{b}}$ & -12 & $-94^{\mathrm{c}},-50^{\mathrm{d}}$ \\
$\mathrm{m} 1$ & -140 & -61 & -155 \\
$\mathrm{~m} 2$ & -136 & -101 & -195 \\
$\mathrm{~m} 3$ & -162 & -100 & -99 \\
$\mathrm{~m} 4$ & -189 & -18 & -179 \\
$\mathrm{UA}$ & -22 & -15 & -52 \\
SAL & -56 & -9 & -26 \\
\hline
\end{tabular}

${ }^{\mathrm{a}}$ Adsorbed through aromatic ring and $\mathrm{C}=\mathrm{C} ;{ }^{\mathrm{b}}$ Adsorbed through $\mathrm{C}=\mathrm{O} ;{ }^{\mathrm{c}}$ via the aromatic ring; ${ }^{\mathrm{d}}$ via both the $\mathrm{C}=\mathrm{C}$ and $\mathrm{C}=\mathrm{O}$ bond. 


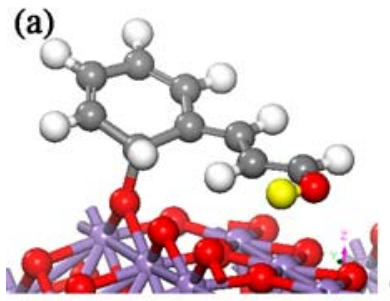

(e)

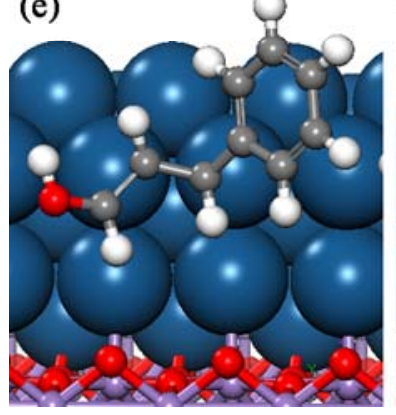

(b)

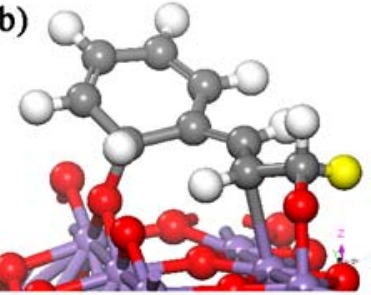

(f)

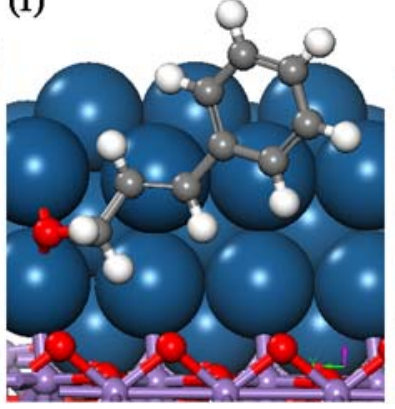

(c)

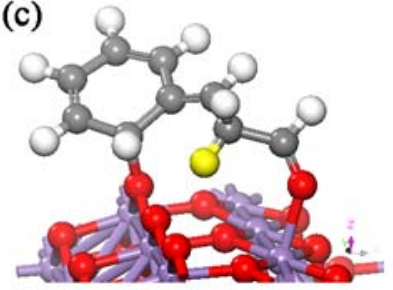

(g)

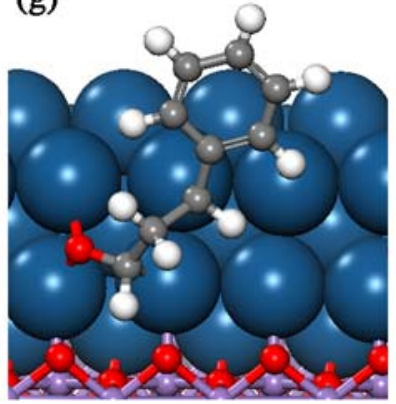

(d)

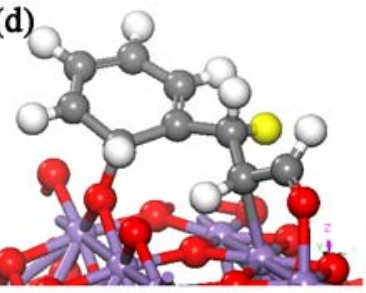

(h)

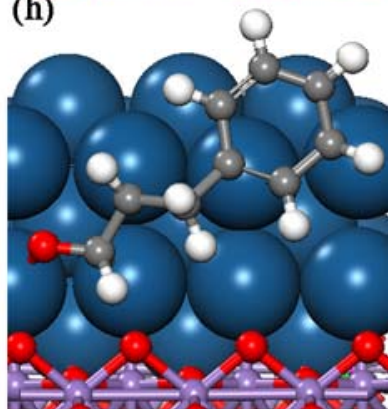

Figure 5.10 Adsorption configurations for the intermediates on the OMS-2 (001) surface for (a) hydroxyl m1; (b) alkoxide m2; (c) alkyl m3; (d) alkyl m4; and on the Pt nanorod supported by OMS-2 for (e) m1; (f) m2; (g) m3; (h) $\mathrm{m} 4$ during cinnamaldehyde hydrogenation.

Table 5.4 DFT calculated activation barriers $\left(\mathrm{kJ} \mathrm{mol}^{-1}\right)$ and reaction energies $\left(\mathrm{kJ} \mathrm{mol}^{-1}\right)$ for the hydrogenation of cinnamaldehyde on Pt(111) surface, bare OMS-2 (001) surface, and the Pt nanorod supported by OMS-2.

\begin{tabular}{c|cc|cc|cc}
\hline \multirow{2}{*}{ Paths } & \multicolumn{2}{|c|}{ Pt(111) surface } & \multicolumn{2}{c|}{ OMS-2 (001) surface } & \multicolumn{2}{c}{ Supported Pt/OMS-2 } \\
\cline { 2 - 7 } & $E_{a c t}$ & $\Delta E_{r x n}$ & $E_{a c t}$ & $\Delta E_{r x n}$ & $E_{a c t}$ & $\Delta E_{r x n}$ \\
\hline a1 (hydroxyl) & 45 & -34 & 136 & 55 & 31 & -60 \\
b1 (alkoxide) & 78 & 69 & 156 & 72 & 65 & 21 \\
c1 (alkyl) & 92 & 30 & 113 & 31 & 84 & 30 \\
d1 (alkyl) & 86 & 13 & 122 & 68 & 79 & -29 \\
\hline a2 & 90 & 44 & 152 & 71 & 72 & 26 \\
b2 & 36 & -60 & 143 & 51 & 30 & -54 \\
c2 & 76 & 2 & 97 & 75 & 74 & -55 \\
d2 & 103 & 17 & 105 & 39 & 88 & 4 \\
\hline
\end{tabular}




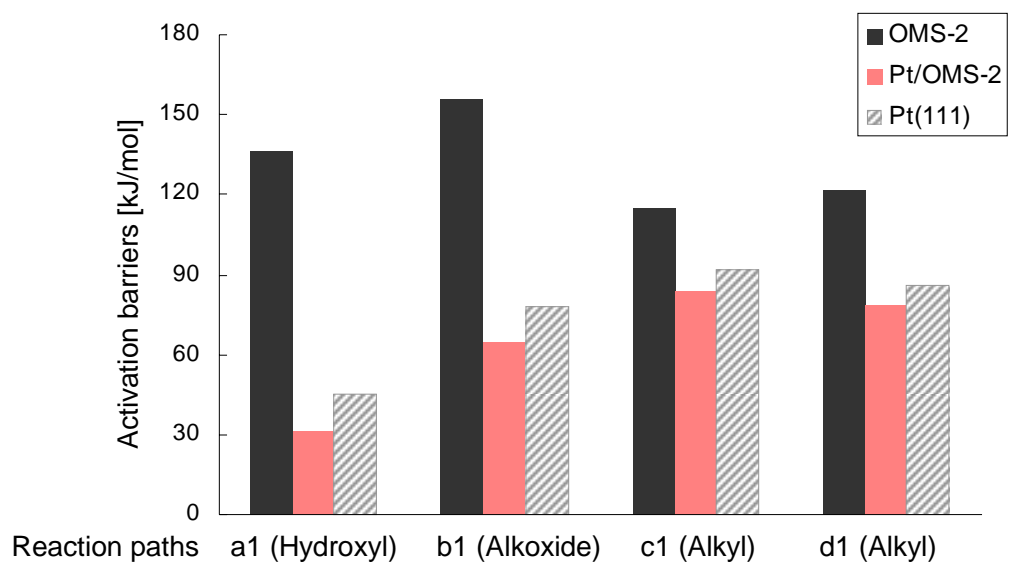

Figure 5.11 Activation barriers $\left(\mathrm{kJ} \mathrm{mol}^{-1}\right)$ for the initial hydrogenation steps of cinnamaldehyde on the OMS-2 (001) surface, Pt(111) surface, and the Pt rod supported by OMS-2.

\section{5) Cinnamaldehyde Hydrogenation over Pt rod supported by OMS-2}

As indicated by Table 5.3, the adsorption of cinnamaldehyde on the supported Pt rod was found to be much stronger than that on the OMS-2 surface. Cinnamaldehyde can adsorb at the interfacial Pt sites of the nanorod either via its aromatic ring (-94 $\mathrm{kJ} \mathrm{mol}^{-1}$, Figure $\left.5.9 \mathrm{~d}\right)$ or via its $\mathrm{C}=\mathrm{C}$ and $\mathrm{C}=\mathrm{O}$ bond in combination $(-50 \mathrm{~kJ}$ $\mathrm{mol}^{-1}$, Figure 5.9e). As cinnamaldehyde preferential adsorbs to the interfacial Pt sites, it is likely hydrogenated at the interfacial Pt/OMS-2 sites rather than on the OMS-2 support alone, which agrees with the temperature programmed desorption (TPD) experiments that observed a large increase in the amount of cinnamaldehyde adsorption after doping OMS-2 with $\mathrm{Pt}[60]$. Here we consider the hydrogenation of the $\mathrm{C}=\mathrm{C}$ and $\mathrm{C}=\mathrm{O}$ bond over the model supported Pt/OMS-2 catalyst starting from the adsorbed state of Figure 5.9e.

The calculated activation barriers over the supported Pt rod $\left(30 \sim 80 \mathrm{~kJ} \mathrm{~mol}^{-1}\right)$, shown in light red in Figure 5.11 and Table 5.4, were found to be significantly lower than those over the OMS-2 surface $\left(110 \sim 150 \mathrm{~kJ} \mathrm{~mol}^{-1}\right.$, shown in grey), and, in addition, lower than those found on Pt (111) surface alone (45 90 kJ mol ${ }^{-1}$ ). As discussed above, the concentration of hydrogen on Pt is higher than that on OMS-2 support. As such it might be proposed that the supported Pt enhances the rate of hydrogenation by lowering the activation barriers as well as increasing hydrogen concentration at Pt perimeter sites as compared to those on the OMS-2 support. This would be consistent with the experimental results which show that the reaction rates are greatly enhanced upon doping OMS-2 with Pt [60].

More specifically, the Pt nanorod/OMS-2 system examined herein appears to greatly favor the hydrogenation of the $\mathrm{C}=\mathrm{O}$ bond to give unsaturated alcohol. The reaction proceeds via the hydroxyl path a1 and has an intrinsic activation barrier of only $31 \mathrm{~kJ} \mathrm{~mol}^{-1}$ which is $100 \mathrm{~kJ} \mathrm{~mol}^{-1}$ lower than that over the OMS-2 surface 
and $15 \mathrm{~kJ} \mathrm{~mol}^{-1}$ lower than that found on the $\mathrm{Pt}(111)$ surface. The barriers to hydrogenate the $\mathrm{C}=\mathrm{C}$ bond on the supported Pt rod are also lower as compared to those on $\operatorname{Pt}(111)$, but the decreases are smaller $\left(7-8 \mathrm{~kJ} \mathrm{~mol}^{-1}\right)$ than those found for the carbonyl group (by 13-15 $\mathrm{kJ} \mathrm{mol}^{-1}$ ). These differences in the reaction energetics can be explained by the electron donation effects from the Pt metal to the OMS-2 support.
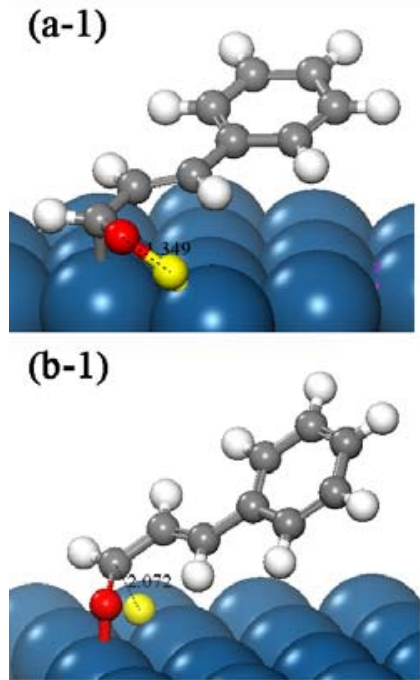

(c-1)

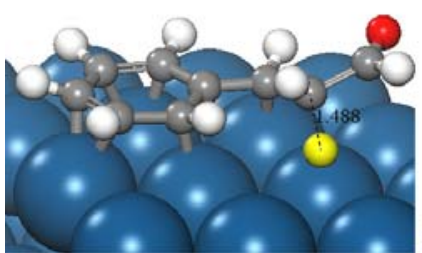

$(\mathrm{d}-1)$

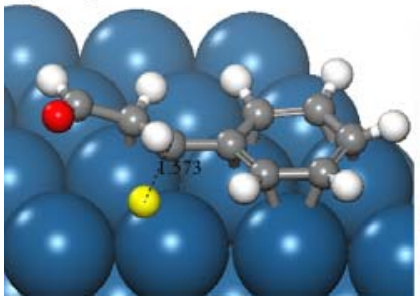

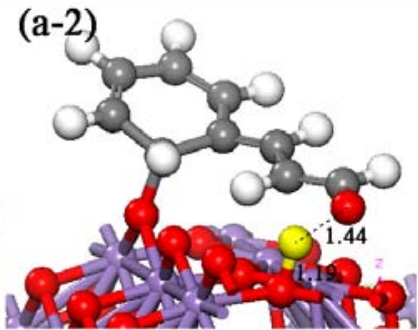

(b-2)

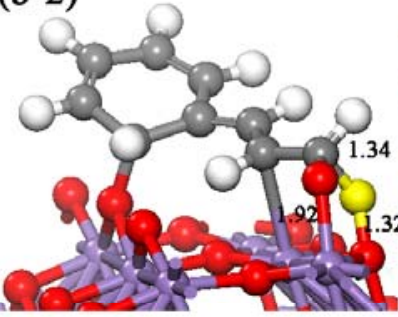

(c-2)

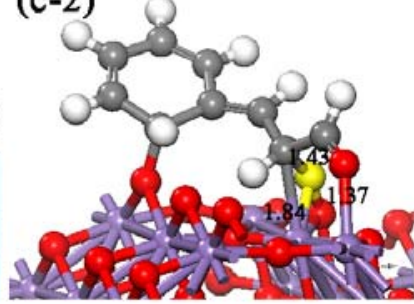

$(\mathrm{d}-2)$

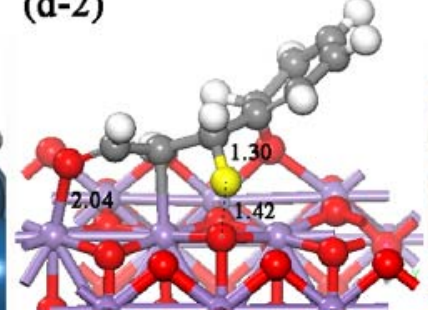

(a-3)

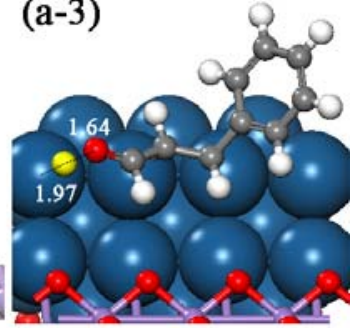

(b-3)
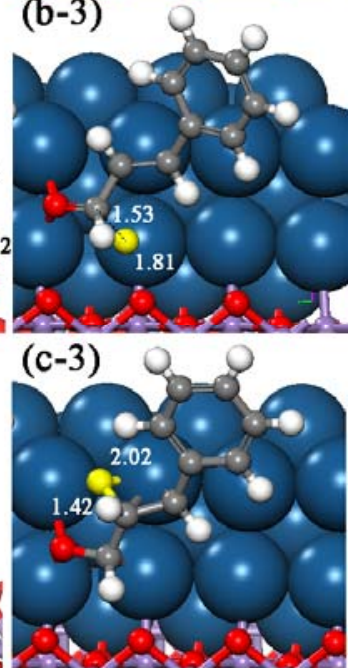

(d-3)

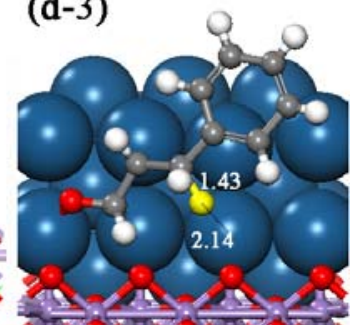

Figure 5.12 Transition state configurations for the hydrogen addition to the $\mathrm{C}=\mathrm{O}$ and $\mathrm{C}=\mathrm{C}$ bonds of cinnamaldehyde on the Pt(111) surface, OMS-2 (001) surface, and the Pt rod supported by OMS-2. (a-1), (a-2), (a-3) are the transition state structures for the hydroxyl path al on Pt(111), OMS-2 surface, and supported Pt/OMS-2, respectively. (b-1), (b-2), (b-3) are the transition state structures for the alkoxide path b1; (c-1), (c-2), (c-3) are the transition state structures for path $\mathrm{c} 1$; and $(\mathrm{d}-1),(\mathrm{d}-2),(\mathrm{d}-3)$ are the transition state structures for path $\mathrm{d} 1$. Bond lengths are in $\AA$. 
The charge density difference map which was shown in Figure 5.5 clearly showed the direct transfer of electrons from the Pt sites to the OMS-2 support at the Pt/OMS-2 interface. As such, the Pt sites adjacent to the support become electron-deficient and take on a partial positive charge. Upon adsorption, the $\mathrm{C}=\mathrm{O}$ group of the cinnamaldehyde reactant can orient itself to allow for electron donation from the lone pair of electrons oxygen to the $\mathrm{Pt}$ site. The cationic $\mathrm{Pt}$ site interacts strongly with the negatively charged $\mathrm{O}$ of the $\mathrm{C}=\mathrm{O}$ group and thus aids in the activation of the $\mathrm{C}=\mathrm{O}$ and the attack by hydrogen (as demonstrated in Figure 5.13). It has also been reported [129-131] that metals with electropositive charge can accelerate the activation of $\mathrm{C}=\mathrm{O}$ bond and promote the rate as well as the selectivity to unsaturated alcohol in hydrogenation reactions.

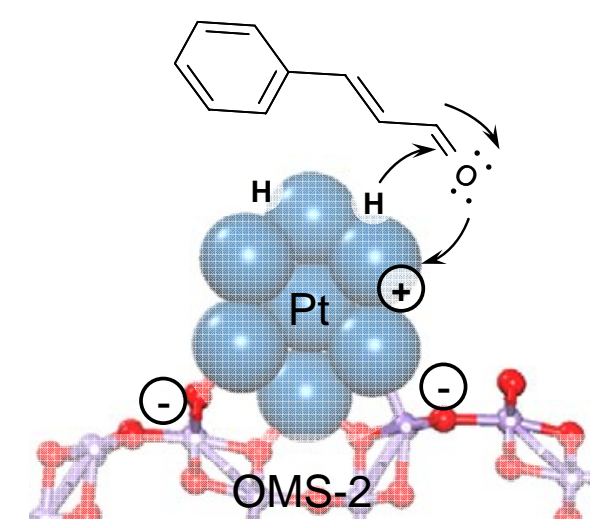

Figure 5.13 Electronic effects that result upon the adsorption and reaction of cinnamaldehyde at the Pt/OMS-2 interface. The $\mathrm{C}=\mathrm{O}$ group of reactant adsorbs via donation of a lone pair of electrons of the oxygen atom.

\section{6) Hydrogenation Activated by the Interfacial Bifunctional Sites}

As discussed above, there is a charge transfer from the interfacial Pt sites to the OMS-2 support, leading to positively charged $\mathrm{Pt}$ sites at the interface that can active the $\mathrm{C}=\mathrm{O}$ bond and accept electrons upon the attack of hydrogen. The specific structural and electronic properties of the OMS-2 support can also help to facilitate the selective reaction at the carbonyl group by providing a bifunctional $\mathrm{Pt}-\mathrm{Mn}$ site that activate the $\mathrm{C}=\mathrm{O}$ bond for hydrogenation. In order to explore the hydrogenation of $\mathrm{C}=\mathrm{O}$ at the bifunctional Pt-Mn sites along the Pt/OMS interface, we model the adsorption of and reaction of $\mathrm{H}_{2} \mathrm{C}=\mathrm{O}$ with hydrogen over Pt/OMS-2 cluster model $\left(\mathrm{Pt}_{10}-\mathrm{H}_{32} \mathrm{Mn}_{12} \mathrm{O}_{40}\right.$, as shown in Figure 5.14) that takes into account the first-order effects to capture the role of $\operatorname{Pt}-\operatorname{Mn}\left(\delta^{+}\right)$bifunctional site.

The $\mathrm{C}=\mathrm{O}$ bond can adsorb at the bifunctional Pt-Mn sites at the Pt-OMS interface with the $\mathrm{C}$ of the $\mathrm{C}=\mathrm{O}$ bond bound to $\mathrm{Pt}$ and the $\mathrm{O}$ coordinated to the $\mathrm{Mn}\left(\delta^{+}\right)$site of the support, thus creating an interfacial $\mathrm{Pt}-(\mathrm{R}) \mathrm{C}=\mathrm{O}-$ $\mathrm{Mn}\left(\delta^{+}\right)$structure. An electron transfers at the transition state from the $\mathrm{Pt}$ to the $\mathrm{C}=\mathrm{O}$ bond together with an 
addition of a Brønsted acid (proton $\mathrm{H}^{+}$) bound to a lattice oxygen of the support, and stabilized by the $\operatorname{Mn}\left(\delta^{+}\right)$ site that acts as a Lewis acid site. This reaction path provides a low activation barrier $\left(19 \mathrm{~kJ} \mathrm{~mol}^{-1}\right)$ for the hydrogenation of the oxygen center of the $\mathrm{C}=\mathrm{O}$ bond with a reaction energy of $-73 \mathrm{~kJ} \mathrm{~mol}^{-1}$. This mechanism is very similar to the model of the $\mathrm{O}=\mathrm{O}$ bond activation at $\mathrm{Au} / \mathrm{TiO} 2$ interface, where the system has an electron transfer from the $\mathrm{Au}$ into the $\mathrm{O}=\mathrm{O}$ bond and a stabilization of the other oxygen by the cationic $\mathrm{Ti}^{4+}$ Lewis acid site [124].
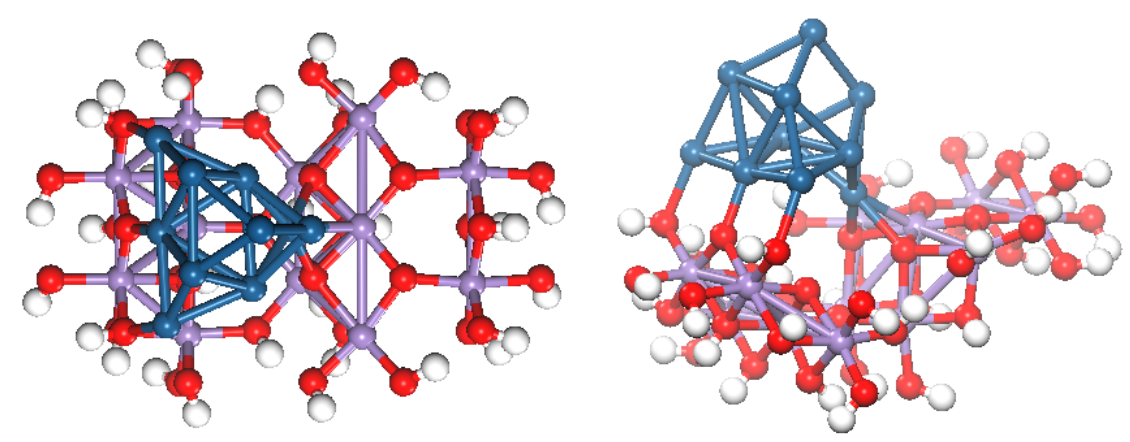

Figure 5.14 Top and side view of cluster model of Pt/OMS-2 interface, $\mathrm{Pt}_{10}-\mathrm{H}_{32} \mathrm{Mn}_{12} \mathrm{O}_{40}$. $\mathrm{Pt}$ atoms are in blue.
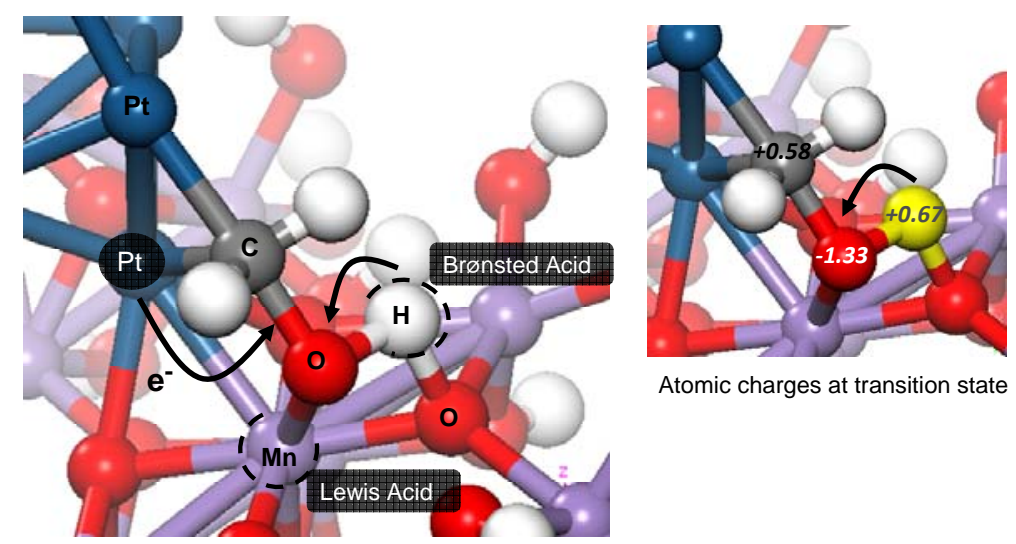

Atomic charges at transition state

Figure 5.15 Atomic charges and the electron transfer that occurs in the hydrogenation of the oxygen of the $\mathrm{C}=\mathrm{O}$ bond, activated by the interfacial $\mathrm{Pt}-\mathrm{Mn}\left(\delta^{+}\right)$bifunctional sites.

The model Pt/OMS-2 interface examined here can therefore efficiently enhance the hydrogenation of $\mathrm{C}=\mathrm{O}$ bond to increase the selectivity to unsaturated alcohol (cinnamyl alcohol) better than the Pt catalyst supported on the conventional oxide supports $[13,18]$, which is also consistent with the experimental results that the $\mathrm{C}=\mathrm{O}$ hydrogenation of cinnamaldehyde is favored on $5 \mathrm{wt} . \% \mathrm{Pt} / \mathrm{OMS}-2$ catalyst to give a $80 \%$ selectivity of UA at conversion of $96 \%[60]$. 


\section{CONCLUSIONS}

The selective hydrogenation of cinnamaldehyde, a model $\alpha, \beta$-unsaturated aldehyde, over novel manganese oxide octahedral molecular sieve (OMS-2) and OMS-2 supported Pt nanorods catalysts was examined using first-principle DFT $+U(+J)$ calculations. Hydrogenation reactions mediated by OMS-2 material were found to proceed but require activation barriers that are $30-100 \mathrm{~kJ} \mathrm{~mol}^{-1}$ higher than those found on the model supported Pt nanorod catalysts. The binding energy of hydrogen to OMS-2 is significantly stronger than on Pt and as such is relatively immobile and much less reactive on OMS-2. The rates of reaction on OMS-2 were therefore much lower than those on Pt catalyst, as observed by experiments. The OMS-2 mediated hydrogenation is likely limited by the dissociation and subsequent migration of hydrogen on the OMS-2 surface. As such, the more thermodynamically stable saturated aldehyde product will be favored over the unsaturated alcohol. The OMS-2 was found to be highly selective for the $\mathrm{C}=\mathrm{C}$ bond hydrogenation to produce saturated aldehyde (SAL), with activation barriers that are much lower than those to hydrogenate the carbonyl for both the first and the second hydrogenation steps.

The adsorption of cinnamaldehyde on the Pt nanorod supported by OMS-2 was found to be much stronger than that on OMS-2 surface, as both the $\mathrm{C}=\mathrm{C}$ and $\mathrm{C}=\mathrm{O}$ bonds can strongly interact with $\mathrm{Pt}$ atoms in combination. In the presence of $\mathrm{Pt}$, the preferential adsorption and the following hydrogenation of cinnamaldehyde appears to preferentially proceed at the interfacial Pt sites rather than on the OMS-2 support, as the calculated activation barriers over supported Pt rod are significantly lower than those over OMS-2. The significant enhancements in rate that result from doping OMS-2 with Pt that are observed in the experiments are thought to be the result of enhanced dissociation and higher mobility of hydrogen on Pt which lead to greater concentration of hydrogen at the active sites at the Pt/OMS-2 interface, as well as the much lower hydrogenation barriers as compared to those on the Pt and OMS-2 surfaces alone. The model supported Pt rod/OMS-2 system greatly favors the selective hydrogenation of the $\mathrm{C}=\mathrm{O}$ bond resulting in the formation of the unsaturated alcohol. This is attributed to the electron donation effects from the Pt metal to the OMS-2 support. The Pt atoms take on positive charge that can accelerate the activation of $\mathrm{C}=\mathrm{O}$ bond by accepting electrons that result during the addition of atomic hydrogen, and thus facilitate the selective hydrogenation to unsaturated alcohol.

The OMS-2 support can also aid the selective hydrogenation at the $\mathrm{C}=\mathrm{O}$ group by providing a bifunctional Pt$\mathrm{Mn}$ sites at the $\mathrm{Pt} / \mathrm{OMS}$ interface where the $\mathrm{C}=\mathrm{O}$ bond binds via its carbon and oxygen atoms to $\mathrm{Pt}$ and $\mathrm{Mn}$ sites, respectively, of the bifunctional Pt-Mn sites. The exposed interfacial Mn sites act as Lewis acid sites and promote $\mathrm{C}=\mathrm{O}$ bond activation. This mechanism provides an activation barrier of only $19 \mathrm{~kJ} \mathrm{~mol}^{-1}$ to form hydroxyl group and can therefore result in significant promotional effects which aid in the selectivity to unsaturated alcohol found experimentally. 
The theoretically calculated activation barriers were found to be in good agreement with experimentally reported values for the hydrogenation of $\mathrm{C}=\mathrm{C}$ bonds, indicating that OMS-2 material is a highly selective catalyst for the $\mathrm{C}=\mathrm{C}$ bond hydrogenation to form saturated aldehyde, in contrast the Pt supported on OMS-2 favors the hydrogenation of the $\mathrm{C}=\mathrm{O}$ bond to give a high selectivity to unsaturated alcohol. The OMS-2 was found to be more important than just supporting the Pt, but can affect the electronic properties of the active metal sites, and in addition, provide Lewis acid sites that can work together with the metal sites to form bifunctional Pt-Mn sites that can selectively hydrogenate $\mathrm{C}=\mathrm{O}$ bonds.

This theoretical study of the model OMS-2 oxide supported metal catalyst, combined with experimental work, help to provide insights into how the OMS-2 materials modify the electronic and structural properties of metal particles to result in significantly enhanced selective hydrogenation of unsaturated aldehydes and ketones into unsaturated alcohols. The results also provide important insights into the efforts into the metal/OMS-2 system as a promising hydrogenation catalyst. The selectivity during hydrogenation of unsaturated oxygenates might may be tailored by an appropriate choice of OMS-2 alone or OMS-2 supported metal catalysts.

\section{Acknowledgment}

We thank Dr. Eric Cockayne of National Institute of Standards and Technology for providing preliminary crystallographic data of the initial structure of K-OMS-2. 


\section{Summary and Recommendations for Future Work}

The work presented in this dissertation provides fundamental insight into the atomic scale features of the reagent, the metal surface, the support as well as the solvent that influence the adsorption and hydrogenation of $\mathrm{C}=\mathrm{O}$ and $\mathrm{C}=\mathrm{C}$ bonds. These factors can be tuned to affect the kinetics and ultimately affect the activity and selectivity in the selective hydrogenation of specific $\alpha, \beta$-unsaturated ketones and aldehydes. Getting a whole picture of the roles of these features is critical for understanding catalytic hydrogenation of many unsaturated oxygenates of interest in fine chemical synthesis and aid in the development of new materials for novel heterogeneous catalysts.

The structure of reagent can affect the electronic characteristics of molecule as well as how it adsorbs onto the surface. For reactants with similar structures, the substitution degree at the $\mathrm{C}=\mathrm{C}$ bond and the chain length of the substituent can influence the overall selectivity on the same catalyst surface. Periodic DFT studies along with microkinetic simulations are used to analyze the reactivity trends of a series of aliphatic $\alpha, \beta$-unsaturated ketones over model $\mathrm{Ru}$ catalyst. Substituents at the $\mathrm{C}=\mathrm{C}$ bond of an unsaturated ketone are found to weaken the adsorption of the reactant near-linearly, lower the rate of $\mathrm{C}=\mathrm{C}$ bond hydrogenation and show notable increase in the production rate of alkoxide intermediate. This, however, is not enough to fully reverse the trends in selectivity as it changes little of the consumption of alkoxide, which is crucial in production of desired unsaturated alcohol (UA). The hydrogenation at $\mathrm{C}=\mathrm{C}$ bond is still more favorable than that of the $\mathrm{C}=\mathrm{O}$ bond on $\mathrm{Ru}$, which rationalizes the observed low selectivity to UA in experiments. The subsequent hydrogenation of the alkoxide to form the unsaturated alcohol is inhibited by the strong Ru-O bond that forms. Increasing the methyl substituents to the $\mathrm{C}=\mathrm{C}$ bond and moving to a less oxophillic metal could significantly increase the selectivity to the unsaturated alcohol.

As the substitution of single methyl at $\mathrm{C}=\mathrm{C}$ bond does not change the rate enough to favor $\mathrm{C}=\mathrm{O}$ hydrogenation, more highly-substituted and complex substrates, benzalacetone and ketoisophorone are investigated. The presence of phenyl group $\alpha$ to $\mathrm{C}=\mathrm{C}$ bond in benzalacetone has hindered the hydrogenation of the olefin $\mathrm{C}=\mathrm{C}$ bond and lower the $\mathrm{C}=\mathrm{C}$ reaction rate, but it still does not alter the barriers enough to favor the UA formation. Hydrogenation at $\mathrm{C}=\mathrm{C}$ bond is still easier and faster than carbonyl, consistent with the results that no observed production of UA in experiments. Although the single substituents such as methyl or aromatic ring cannot achieve the goal to lower the $\mathrm{C}=\mathrm{C}$ reaction rate to compete with $\mathrm{C}=\mathrm{O}$ hydrogenation, the addition of multiple substitutions at the hydrocarbon backbone can begin to alter the selectivity. The methyl substituent along with the presence of a second $\mathrm{C}=\mathrm{O}$ group in ketoisophorone (KIP) act to limit hydrogen access to both the carbons of $\mathrm{C}=\mathrm{C}$ bond and so as to enhance the rate of $\mathrm{C}=\mathrm{O}$ hydrogenation over $\mathrm{C}=\mathrm{C}$ hydrogenation. The branching 
alkyl substitutions around the hindered carbonyl group were found to help to distribute and stabilize the partial charges at transition state and promote its hydrogenation, while the other unhindered carbonyl is left untouched. The high selectivity to UA formed in ketoisophorone hydrogenation, compared to the completed lack of UA in the hydrogenation of aliphatic ketones such as MVK, IMK, and benzalacetone, illustrates the importance of multiple substitutions and substituent positions.

The interaction between reactant and metal surface is very sensitive to the interfacial structure which is subjected to the co-operative effects of the metal and specific structure of reactant. Hydrogenations of benzalacetone and KIP are investigated on model Pt, Pd and Ru catalysts to establish the effects of metal. The differences in the rates of benzalacetone hydrogenation on $\mathrm{Pt}$ and $\mathrm{Ru}$ are closely related to the binding strength of reactant, intermediates and products. Over $\mathrm{Pt}$ the barriers for the first hydrogenation of $\mathrm{C}=\mathrm{O}$ bond are considerably higher than that to hydrogenate $\mathrm{C}=\mathrm{C}$ bond. Calculated barriers are significantly higher over $\mathrm{Ru}$ compared to Pt, which is consistent with the lower experimental TOF reported over Ru than Pt. For the KIP hydrogenation Pt is predicted to produce almost the same amount of UA and SK, while Pd is more selective for the reduction of sterically-hindered $\mathrm{C}=\mathrm{O}$ bond and leads to higher selectivity to $\mathrm{UA}$ as compared to Pt. The calculated adsorption energies and the intrinsic reaction energies and activation barriers help to understand the negligible selectivity to UA in benzalacetone hydrogenation reported over supported $\mathrm{Pt}$ and Ru catalysts as well as the markedly different selectivities to UA found in the hydrogenation of KIP over supported Pd and Pt catalysts.

Solvent can affect the reaction rate and selectivity via cooperative effects such as hydrogen bonding, solvation and the transfer of protons. Polar protic solvents, such as water and 10\% mixed isopropyl alcohol (IPA)-water solvent, are found to lower the activation barriers by stabilizing the partial charged transition state, and alter the preferred reaction mechanism by directly participating in the hydrogenation of unsaturated aldehyde acrolein on model Pt catalysts. The ab initio molecular dynamics simulations are used to determine the favorable structures of metal-solvent interface, where the acrolein binds and forms hydrogen bonds with solvent molecules within its local solvation shell. Both water and the mixed solvent enhance the $\mathrm{C}=\mathrm{O}$ bond hydrogenation via the hydroxyl hydrogenation path, and charge analysis suggests that the charge distribution in the transition state stabilizes the metal-mediated path observed in mixed solvent over that carried out in water. The solvent-mediated proton shuttling pathway largely depends on the strength of the hydrogen bonds and is expected to be dominant for the acrolein hydrogenation in water. Theoretical results correlate well with the experimental observed increased selectivity to UA in 10\% mixed IPA-water solvent and shed light to the possibility to tailor catalytic performance through controlling the composition of solvent.

The non-polar aromatic solvent such as toluene and substituted aromatics do not provide hydrogen bonding as the protic solvents did but can affect the reaction through changing the surface property of catalyst by blocking 
the surface sites via the strong binding. Toluene is found to adsorb on the Pt surface, block the surface sites and thus inhibit the adsorption and ring hydrogenation of benzylacetone reactant. At higher concentration of toluene, benzylacetone can adsorb only via its $\mathrm{C}=\mathrm{O}$ group in a vertical mode at exposed $\mathrm{Pt}$ sites between adsorbed toluene intermediates. Selectivity to UA increases greatly as the only available path for hydrogenation is via the $\mathrm{C}=\mathrm{O}$ bond. The bulky side chain of tert-butyl toluene limits its ability to efficiently block surface and thus lead to a higher rate but more products from ring hydrogenation of benzylacetone.

The presence of solvent stabilizes the partial-charged transition states which increases the rates of carbonyl hydrogenation and provide paths which facilitate the $\mathrm{C}=\mathrm{O}$ reduction. The support can potentially also play a similar role in terms of modifying electronic properties and providing additional functionality which can enhance the $\mathrm{C}=\mathrm{O}$ hydrogenation over that of $\mathrm{C}=\mathrm{C}$ bond. Novel manganese oxide octahedral molecular sieve (OMS-2) material is found to be highly selective for $\mathrm{C}=\mathrm{C}$ bond hydrogenation of cinnamaldehyde to form saturated aldehyde. However the hydrogenations mediated by OMS-2 require significantly high activation barriers as the hydrogen bond on OMS-2 is relatively immobile and as such less reactive. The OMS-2 material can also act as support to anchor Pt particles which can readily split hydrogen and provide possible bifunctional sites that may improve selective hydrogenation. The Pt nanorod supported on OMS-2 was found to favor the $\mathrm{C}=\mathrm{O}$ hydrogenation to give a high selectivity to unsaturated alcohol, which can be attributed to the electron transfer effects from Pt to the OMS-2 support leading to positively charged Pt ions at the interface that can active the $\mathrm{C}=\mathrm{O}$ bond for accept the attack from hydrogen. The OMS-2 support can also aid the selective hydrogenation of $\mathrm{C}=\mathrm{O}$ bond by providing a bifunctional Pt-Mn site at the $\mathrm{Pt} / \mathrm{OMS}$ interface. The Mn site may work as a Lewis acid site and activate the $\mathrm{C}=\mathrm{O}$ bond for hydrogenation which leads to the promotional effects to unsaturated alcohol that was found experimentally. OMS-2 is thus thought to promote Pt and as well directly participate in the catalytic reaction. The selectivity in the hydrogenation of unsaturated oxygenates then may be tailored by an appropriate choice of OMS-2 alone or the OMS-2 supported metal catalysts.

The possible future work may extend to: 1) the solvent (water) phase hydrogenation over OMS-2 based catalysts. In this dissertation we have proposed from the binding energy and the barriers of hydrogen spillover that the OMS-2 mediated hydrogenation was likely to be limited by the slow spillover of hydrogen. Water may serve to assist the dissociation of hydrogen over OMS-2, increase the hydrogen concentration on OMS-2 and thus accelerate the hydrogenation reactions. 2) The solvent-phase kinetic Monte Carlo simulations, if possible, will help to predict the rate limiting step during the liquid phase hydrogenation and ultimately locate the favorable pathway or mechanisms which will provide useful correlations to the current theoretical results to compare with known experimental observations. 3) Consider the influence of accumulated adsorbed products. The accumulation of the adsorbed unsaturated alcohol or saturated ketone/aldehyde products formed during the hydrogenation on the surface of the catalyst may modify the surface and hence alter the outcome of the 
reaction. The equilibrium adsorption amount of reactant can also be affected by the accumulated products on the surface, especially for the substrate with the complex molecular structures. The effects of increased coverage of products could be studied by doping or pre-adsorption of alcohol or ketone, aldehyde species on the catalyst surface to understand their influence on the adsorption behavior of reaction intermediates and the following hydrogenation steps. 


\section{References}

[1] V. Ponec, On the role of promoters in hydrogenations on metals; alpha,beta-unsaturated aldehydes and ketones, Applied Catalysis a-General, 149 (1997) 27-48.

[2] R.A. Sheldon, H. van Bekkum, Fine Chemicals through Heterogeneous Catalysis, Weinheim: WILEY$\mathrm{VCH},(2001)$.

[3] P.N. Rylander, Hydrogenation in Organic Synthesis, Academic Press, New York, (1979).

[4] R.L. Augustine, Heterogeneous Catalysts for the Synthetic Chemist., Marcel Dekker Inc., New York, (1996).

[5] A. Chambers, S.D. Jackson, D. Stirling, G. Webb, Selective hydrogenation of cinnamaldehyde over supported copper catalysts, Journal of Catalysis, 168 (1997) 301-314.

[6] U.K. Singh, M.A. Vannice, Kinetics of liquid-phase hydrogenation reactions over supported metal catalysts - a review, Applied Catalysis a-General, 213 (2001) 1-24.

[7] J.N. Chheda, G.W. Huber, J.A. Dumesic, Liquid-phase catalytic processing of biomass-derived oxygenated hydrocarbons to fuels and chemicals, Angew. Chem.-Int. Edit, 46 (2007) 7164-7183.

[8] P. Gallezot, D. Richard, Selective hydrogenation of alpha,beta-unsaturated aldehydes, Catalysis ReviewsScience and Engineering, 40 (1998) 81-126.

[9] M. Gargano, V. Dorazio, N. Ravasio, M. Rossi, Selective Preparation of Unsaturated Alcohols Via Catalytic Meerwein-Ponndorf Reduction of Unsaturated Carbonyl-Compounds over Metal-Oxides, Journal of Molecular Catalysis, 58 (1990) L5-L8.

[10] T. Ohkuma, H. Ikehira, T. Ikariya, R. Noyori, Asymmetric hydrogenation of cyclic alpha,beta-unsaturated ketones to chiral allylic alcohols, Synlett, (1997) 467-469.

[11] S.N. Coman, V.I. Parvulescu, M. De Bruyn, D.E. De Vos, P.A. Jacobs, Reduction of prostaglandin unsaturated ketones to secondary allylic alcohols by hydrogen transfer over mesoporous-supported PtSn catalysts, Journal of Catalysis, 206 (2002) 218-229.

[12] P. Maki-Arvela, J. Hajek, T. Salmi, D.Y. Murzin, Chemoselective hydrogenation of carbonyl compounds over heterogeneous catalysts, Applied Catalysis a-General, 292 (2005) 1-49.

[13] M.S. Ide, B. Hao, M. Neurock, R.H. Davis, Mechanistic insights on the hydrogenation of $\alpha, \beta$-unsaturated ketones and aldehydes to unsaturated alcohols over metal catalysts, ACS catalysis, 2 (2012) 671-683.

[14] T.B.L.W. Marinelli, S. Nabuurs, V. Ponec, Activity and selectivity in the reactions of substituted $\alpha, \beta$ unsaturated aldehydes, Journal of Catalysis, 151 (1995) 431-438.

[15] C. Hoang-Van, O. Zegaoui, Studies of high surface area Pt/MoO3 and Pt/WO3 catalysts for selective hydrogenation reactions. II. Reactions of acrolein and allyl alcohol, Applied Catalysis A: General, 164 (1997) 91-103. 
[16] C. Milone, R. Ingoglia, A. Pistone, G. Neri, F. Frusteri, S. Galvagno, Selective hydrogen of alpha, betaunsaturated ketones to alpha, beta-unsaturated alcohols on gold-supported catalysts, Journal of Catalysis, 222 (2004) 348-356.

[17] C. Milone, R. Ingoglia, M.L. Tropeano, G. Neri, S. Galvagno, First example of selective hydrogenation of unconstrained alpha,beta-unsaturated ketone to alpha,beta-unsaturated alcohol by molecular hydrogen, Chemical Communications, (2003) 868-869.

[18] M. Lashdaf, A.O.I. Krause, M. Lindblad, A. Tiitta, T. Venalainen, Behaviour of palladium and ruthenium catalysts on alumina and silica prepared by gas and liquid phase deposition in cinnamaldehyde hydrogenation, Applied Catalysis a-General, 241 (2003) 65-75.

[19] M. Lashdaf, A. Hase, E. Kauppinen, A.O.I. Krause, Fullerene-based ruthenium catalysts in cinnamaldehyde hydrogenation, Catalysis Letters, 52 (1998) 199-204.

[20] S. Galvagno, G. Capannelli, G. Neri, A. Donato, R. Pietropaolo, Hydrogenation of cinnamaldehyde over $\mathrm{Ru} / \mathrm{C}$ catalysts - effect of Ru particle-size, Journal of Molecular Catalysis, 64 (1991) 237-246.

[21] S. Galvagno, C. Milone, A. Donato, G. Neri, R. Pietropaolo, Influence of metal-particle size in the hydrogenation of citral over Ru/C, Catalysis Letters, 18 (1993) 349-355.

[22] L. Mercadante, G. Neri, C. Milone, A. Donato, S. Galvagno, Hydrogenation of alpha,beta-unsaturated aldehydes over Ru/A12O3 catalysts, Journal of Molecular Catalysis a-Chemical, 105 (1996) 93-101.

[23] B. Coq, P.S. Kumbhar, C. Moreau, P. Moreau, M.G. Warawdekar, Liquid-Phase Hydrogenation of Cinnamaldehyde over Supported Ruthenium Catalysts - Influence of Particle-Size, Bimetallics and Nature of Support, Journal of Molecular Catalysis, 85 (1993) 215-228.

[24] M. Englisch, V.S. Ranade, J.A. Lercher, Liquid phase hydrogenation of crotonaldehyde over $\mathrm{Pt} / \mathrm{SiO}_{2}$ catalysts, Applied Catalysis a-General, 163 (1997) 111-122.

[25] A. Dandekar, M.A. Vannice, Crotonaldehyde hydrogenation on $\mathrm{Pt} / \mathrm{TiO}_{2}$ and $\mathrm{Ni} / \mathrm{TiO}_{2} \mathrm{SMSI}$ catalysts, Journal of Catalysis, 183 (1999) 344-354.

[26] M. Arai, A. Obata, K. Usui, M. Shirai, Y. Nishiyama, Activity for liquid-phase hydrogenation of alpha,beta-unsaturated aldehydes of supported platinum catalysts prepared through low-temperature reduction, Applied Catalysis a-General, 146 (1996) 381-389.

[27] M. Arai, K. Usui, Y. Nishiyama, Preparation of Alumina-Supported Platinum Catalyst at AmbientTemperature for Selective Synthesis of Cinnamyl Alcohol by Liquid-Phase Cinnamaldehyde Hydrogenation, Journal of the Chemical Society-Chemical Communications, (1993) 1853-1854.

[28] U.K. Singh, M.A. Vannice, Liquid-phase citral hydrogenation over $\mathrm{SiO}_{2}$-supported group VIII metals, Journal of Catalysis, 199 (2001) 73-84.

[29] P.N. Rylander, Catalytic Hydrogenation, Academic Press, London, (1985).

[30] H. Yamada, S. Goto, The effect of solvents polarity on selective hydrogenation of unsaturated aldehyde in gas-liquid-solid three phase reactor, Journal of Chemical Engineering of Japan, 36 (2003) 586-589. 
[31] B.S. Akpa, C. D'Agostino, L.F. Gladden, K. Hindle, H. Manyar, J. McGregor, R. Li, M. Neurock, N. Sinha, E.H. Stitt, D. Weber, J.A. Zeitler, D.W. Rooney, Solvent effects in the hydrogenation of 2butanone, Journal of Catalysis, 289 (2012) 30-41.

[32] S. Mukherjee, M.A. Vannice, Solvent effects in liquid-phase reactions - I. Activity and selectivity during citral hydrogenation on $\mathrm{Pt} / \mathrm{SiO}_{2}$ and evaluation of mass transfer effects, Journal of Catalysis, 243 (2006) 108-130.

[33] A.D. Aguirre, P. Reyes, M. Oportus, I. Melian-Cabrera, J.L.G. Fierro, Liquid phase hydrogenation of crotonaldehyde over bimetallic $\mathrm{Rh}-\mathrm{Sn} / \mathrm{SiO}_{2}$ catalysts - Effect of the $\mathrm{Sn} / \mathrm{Rh}$ ratio, Applied Catalysis aGeneral, 233 (2002) 183-196.

[34] R. Hirschl, F. Delbecq, P. Sautet, J. Hafner, Adsorption of unsaturated aldehydes on the (111) surface of a Pt-Fe alloy catalyst from first principles, Journal of Catalysis, 217 (2003) 354-366.

[35] F. Delbecq, P. Sautet, A density functional study of adsorption structures of unsaturated aldehydes on Pt(111): A key factor for hydrogenation selectivity, Journal of Catalysis, 211 (2002) 398-406.

[36] D. Loffreda, F. Delbecq, P. Sautet, Adsorption thermodynamics of acrolein on Pt (1 111 1 $)$ in realistic temperature and pressure from first-principle calculations, Chemical Physics Letters, 405 (2005) 434439.

[37] G. Kang, Z. Chen, Z. Li, Acrolein adsorption on gold clusters, a theoretical study of conjugation effect on $\mathrm{C}=\mathrm{C}$ and $\mathrm{C}=\mathrm{O}$ interaction with Au clusters, Catalysis Letters, 141 (2011) 996-1003.

[38] R. Hirschl, A. Eichler, J. Hafner, Hydrogenation of ethylene and formaldehyde on $\operatorname{Pt}(111)$ and Pt80Fe20(111): a density-functional study, Journal of Catalysis, 226 (2004) 273-282.

[39] D. Loffreda, F. Delbecq, F. Vigne, P. Sautet, Catalytic hydrogenation of unsaturated aldehydes on Pt (111): understanding the selectivity from first-principles calculations, Angewandte Chemie International Edition, 44 (2005) 5279-5282.

[40] K.H. Lim, A.B. Mohammad, I.V. Yudanov, K.M. Neyman, M. Bron, P. Claus, N. Rosch, Mechanism of selective hydrogenation of alpha,beta-unsaturated aldehydes on silver catalysts: a sensity functional study, Journal of Physical Chemistry C, 113 (2009) 13231-13240.

[41] R. Hirschl, F. Delbecq, P. Sautet, J. Hafner, $\mathrm{Pt}_{80} \mathrm{Fe}_{20}$ surface from first principles: Electronic structure and adsorption of CO and atomic H, Physical Review B, 66 (2002) 155438-155449.

[42] B.C. Khanra, Y. Jugnet, J.C. Bertolini, Energetics of acrolein hydrogenation on $\operatorname{Pt}\left(\begin{array}{lll}1 & 1 & 1\end{array}\right)$ and $\operatorname{Ag}\left(\begin{array}{lll}1 & 1 & 1\end{array}\right)$ surfaces: a BOC-MP model study, Journal of Molecular Catalysis A: Chemical, 208 (2004) 167-174.

[43] D. Loffreda, F. Delbecq, F. Vigne, P.J. Sautet, Chemo-regioselectivity in heterogeneous catalysis: competitive routes for $\mathrm{CO}$ and $\mathrm{CC}$ hydrogenations from a theoretical approach, Journal of the American Chemical Society, 128 (2006) 1316-1323.

[44] S. Laref, F. Delbecq, D. Loffreda, Theoretical elucidation of the selectivity changes for the hydrogenation of unsaturated aldehydes on Pt(111), Journal of Catalysis, 265 (2009) 35-42. 
[45] N. Sinha, M. Neurock, A first principles analysis of the hydrogenation of $\mathrm{C}_{1}-\mathrm{C}_{4}$ aldehydes and ketones over Ru(0001), Journal of Catalysis, 295 (2012) 31-44.

[46] M. Steffan, M. Lucas, A. Brandner, M. Wollny, N. Oldenburg, P. Claus, Selective hydrogenation of citral in an organic solvent, in a ionic liquid, and in substance, Chemical Engineering \& Technology, 30 (2007) 481-486.

[47] G.M.R. vanDruten, L. Aksu, V. Ponec, On the promotion effects in the hydrogenation of acetone and propanal, Applied Catalysis a-General, 149 (1997) 181-187.

[48] S. Galvagno, C. Milone, A. Donato, G. Neri, R. Pietropaolo, Hydrogenation of Citral over Ru-Sn/C, Catalysis Letters, 17 (1993) 55-61.

[49] S. Galvagno, C. Milone, G. Neri, A. Donato, R. Pietropaolo, Hydrogenation of Cinnamaldehyde and Citral over Ru Supported Catalysts, in: M. Guisnet, J. Barbier, J. Barrault, C. Bouchoule, D. Duprez, G. Perot, C. Montassier (Eds.) Heterogeneous Catalysis and Fine Chemicals Iii, 1993, pp. 163-170.

[50] G. Neri, C. Milone, A. Donato, L. Mercadante, A.M. Visco, Selective Hydrogenation of Citral over Pt-Sn Supported on Activated Carbon, Journal of Chemical Technology and Biotechnology, 60 (1994) 83-88.

[51] D. Loffreda, F. Delbecq, F. Vigne, P. Sautet, Catalytic hydrogenation of unsaturated aldehydes on Pt(111): Understanding the selectivity from first-principles calculations, Angewandte Chemie-International Edition, 44 (2005) 5279-5282.

[52] D. Loffreda, F. Delbecq, F. Vigne, P. Sautet, Chemo-regioselectivity in heterogeneous catalysis: Competitive routes for $\mathrm{C}=\mathrm{O}$ and $\mathrm{C}=\mathrm{C}$ hydrogenations from a theoretical approach, Journal of the American Chemical Society, 128 (2006) 1316-1323.

[53] H. Takagi, T. Isoda, K. Kusakabe, S. Morooka, Effects of solvents on the hydrogenation of monoaromatic compounds using noble-metal catalysts, Energy \& Fuels, 13 (1999) 1191-1196.

[54] H. Daly, A. Elliott, C. Hardacre, H. Manyar, I. McManus, R. Morgan, D. Rooney, J. Thompson, Study of solvent effects in liquid phase hydrogenation reactions. Queen's University, Belfast, 2012, In preparation.

[55] S. Dharmarathna, C.K. King'ondu, W. Pedrick, L. Pahalagedara, S.L. Suib, Direct Sonochemical Synthesis of Manganese Octahedral Molecular Sieve (OMS-2) Nanomaterials Using Cosolvent Systems, Their Characterization, and Catalytic Applications, Chemistry of Materials, 24 (2012) 705-712.

[56] C.K. King'ondu, N. Opembe, C.-h. Chen, K. Ngala, H. Huang, A. Iyer, H.F. Garces, S.L. Suib, Manganese Oxide Octahedral Molecular Sieves (OMS-2) Multiple Framework Substitutions: A New Route to OMS-2 Particle Size and Morphology Control, Advanced Functional Materials, 21 (2011) 312323.

[57] G. Qiu, H. Huang, S. Dharmarathna, E. Benbow, L. Stafford, S.L. Suib, Hydrothermal Synthesis of Manganese Oxide Nanomaterials and Their Catalytic and Electrochemical Properties, Chemistry of Materials, 23 (2011) 3892-3901. 
[58] V.D. Makwana, Y.C. Son, A.R. Howell, S.L. Suib, The role of lattice oxygen in selective benzyl alcohol oxidation using OMS-2 catalyst: A kinetic and isotope-labeling study, Journal of Catalysis, 210 (2002) 46-52.

[59] G.D. Yadav, H.G. Manyar, Synthesis of a Novel Redox Material UDCaT-3: An Efficient and Versatile Catalyst for Selective Oxidation, Hydroxylation and Hydrogenation Reactions, Advanced Synthesis \& Catalysis, 350 (2008) 2286-2294.

[60] H.G. Manyar, B. Yang, H. Daly, H. Moor, S. McMonagle, Y. Tao, G.D. Yadav, A. Goguet, P. Hu, C. Hardacre, Selective Hydrogenation of $\alpha, \beta$-unsaturated Aldehydes and Ketones using Novel Manganese Oxide and Platinum supported on Manganese Oxide Octahedral Molecular Sieves as Catalysts, ChemCatChem, 5 (2013) 506-512.

[61] P. Hohenberg, W. Kohn, Inhomogeneous electron gas, Physical Review B, 136 (1964) 864-871.

[62] W. Kohn, L.J. Sham, Self-consistent equations including exchange and correlation effects Physical Review, 140 (1965) 1133-1138.

[63] G.C. Bond, Catalysis of Metals London: Academic Press, (1962).

[64] P. Kluson, L. Cerveny, Selective Hydrogenation over Ruthenium Catalysts, Applied Catalysis a-General, 128 (1995) 13-31.

[65] M. Neurock, V. Pallassana, R.A. van Santen, The importance of transient states at higher coverages in catalytic reactions, Journal of the American Chemical Society, 122 (2000) 1150-1153.

[66] P.N. Rylander, Catalytic Hydrogenation, Academic Press, London, (1985).

[67] N. Sinha, Ph.D. Dissertation, University of Virginia, (2009).

[68] G. Kresse, J. Furthmuller, Efficient iterative schemes for ab initio total-energy calculations using a planewave basis set, Physical Review B, 54 (1996) 11169-11186.

[69] J.P. Perdew, J.A. Chevary, S.H. Vosko, K.A. Jackson, M.R. Pederson, D.J. Singh, C. Fiolhais, Atoms, Molecules, Solids, and Surfaces - Applications of the Generalized Gradient Approximation for Exchange and Correlation, Physical Review B, 46 (1992) 6671-6687.

[70] D. Vanderbilt, Optimally Smooth Norm-Conserving Pseudopotentials, Physical Review B, 32 (1985) 8412-8415.

[71] H.J. Monkhorst, J.D. Pack, Special Points for Brillouin-Zone Integrations, Physical Review B, 13 (1976) 5188-5192.

[72] M. Methfessel, A.T. Paxton, High-Precision Sampling for Brillouin-Zone Integration in Metals, Physical Review B, 40 (1989) 3616-3621.

[73] Structure Data of Elements and Intermetallic Phases, Landolt-Börnstein New Series, Group B, Vol. III, Springer-Verlag, Berlin (1971).

[74] G. Mills, H. Jonsson, G.K. Schenter, Reversible Work Transition-State Theory - Application to Dissociative Adsorption of Hydrogen, Surface Science, 324 (1995) 305-337. 
[75] H. Jónsson, G. Mills, K.W. Jacobsen, Nudged Elastic Band Method for Finding Minimum Energy Paths of Transitions, in Classical and Quantum Dynamics in Condensed Phase Simulations, World Scientific, Singapore, (1998).

[76] G. Henkelman, H. Jonsson, Improved tangent estimate in the nudged elastic band method for finding minimum energy paths and saddle points, Journal of Chemical Physics, 113 (2000) 9978-9985.

[77] D. Sheppard, R. Terrell, G. Henkelman, Optimization methods for finding minimum energy paths, Journal of Chemical Physics, 128 (2008).

[78] G. Henkelman, H. Jonsson, A dimer method for finding saddle points on high dimensional potential surfaces using only first derivatives, Journal of Chemical Physics, 111 (1999) 7010-7022.

[79] A. Heyden, A.T. Bell, F.J. Keil, Efficient methods for finding transition states in chemical reactions: Comparison of improved dimer method and partitioned rational function optimization method, Journal of Chemical Physics, 123 (2005).

[80] J. Kaestner, P. Sherwood, Superlinearly converging dimer method for transition state search, Journal of Chemical Physics, 128 (2008).

[81] K.H. Lim, A.B. Mohammad, I.V. Yudanov, K.M. Neyman, M. Bron, P. Claus, N. Rosch, Mechanism of Selective Hydrogenation of alpha,beta-Unsaturated Aldehydes on Silver Catalysts: A Density Functional Study, Journal of Physical Chemistry C, 113 (2009) 13231-13240.

[82] L. Xu, H.Y. Xiao, X.T. Zu, Hydrogen adsorption on $\mathrm{Ru}(001)$ surface from density-functional periodic calculations, Chemical Physics, 315 (2005) 155-160.

[83] N.K. Sinha, Master Thesis, Chapter3, Department of Chemical Engineering, University of Virginia, Charlottesville, (2008).

[84] M.G. Evans, M. Polanyi, Inertia and driving force of chemical reactions, Transactions of the Faraday Society, 34 (1938) 11.

[85] Z. Belohlav, P. Zamostny, A rate-controlling step in Langmuir-Hinshelwood kinetic models, Canadian Journal of Chemical Engineering, 78 (2000) 513-521.

[86] J.E. Rekoske, R.D. Cortright, S.A. Goddard, S.B. Sharma, J.A. Dumesic, Microkinetic analysis of diverse experimental-data for ethylene hydrogenation on platinum, Journal of Physical Chemistry, 96 (1992) 1880-1888.

[87] D. Mei, M. Neurock, C.M. Smith, Hydrogenation of acetylene-ethylene mixtures over Pd and Pd-Ag alloys: First-principles-based kinetic Monte Carlo simulations, Journal of Catalysis, 268 (2009) 181-195.

[88] D.H. Mei, E.W. Hansen, M. Neurock, Ethylene hydrogenation over bimetallic $\mathrm{Pd} / \mathrm{Au}(111)$ surfaces: Application of quantum chemical results and dynamic Monte Carlo simulation, Journal of Physical Chemistry B, 107 (2003) 798-810.

[89] R. Rao, A. Dandekar, R.T.K. Baker, M.A. Vannice, Properties of copper chromite catalysts in hydrogenation reactions, Journal of Catalysis, 171 (1997) 406-419. 
[90] K.H. Lim, A.B. Mohammad, I.V. Yudanov, K.M. Neyman, M. Bron, P. Claus, N. Roesch, Mechanism of Selective Hydrogenation of alpha,beta-Unsaturated Aldehydes on Silver Catalysts: A Density Functional Study, Journal of Physical Chemistry C, 113 (2009) 13231-13240.

[91] M. von Arx, T. Mallat, A. Baiker, Unprecedented selectivity behaviour in the hydrogenation of an alpha,beta-unsaturated ketone: hydrogenation of ketoisophorone over alumina-supported $\mathrm{Pt}$ and $\mathrm{Pd}$, Journal of Molecular Catalysis a-Chemical, 148 (1999) 275-283.

[92] N.W. Ashcroft, N.D. Mermin, Solid State Physics; Holt, Rinehart and Winston: New York, 1976.

[93] N. Landolt-Börnstein, Structure Data of Elements and Intermetallic Phases: Springer-Verlag: Berlin, 1971.

[94] N. Sinha, Ph.D. Dissertation, University of Virginia, May 2009.

[95] R. Schmid, E.A. Broger, M. Cereghetti, Y. Crameri, J. Foricher, M. Lalonde, R.K. Muller, M. Scalone, G. Schoettel, U. Zutter, New developments in enantioselective hydrogenation, Pure and Applied Chemistry, 68 (1996) 131-138.

[96] H. Brunner, K. Fisch, Asymmetric Catalysis .82. Enantioselective Hydration of 4-Oxoisophorone, Journal of Organometallic Chemistry, 456 (1993) 71-75.

[97] N.S. Zarghami, D.E. Heinz, Monoterpene aldehydes and isophorone-related compounds of saffron, Phytochemistry, 10 (1971) 2755-2761.

[98] P.A. Tarantilis, M.G. Polissiou, Isolation and identification of the aroma components from saffron (Crocus sativus), Journal of Agricultural and Food Chemistry, 45 (1997) 459-462.

[99] Y.A. Ryndin, C.C. Santini, D. Prat, J.M. Basset, Chemo-, regio-, and diastereoselective hydrogenation of oxopromegestone into Trimegestone over supported platinoids: Effects of the transition metal, support nature, tin additives, and modifiers, Journal of Catalysis, 190 (2000) 364-373.

[100] W. Tang, E. Sanville, G. Henkelman, A grid-based Bader analysis algorithm without lattice bias, Journal of Physics-Condensed Matter, 21 (2009).

[101] J. Wood, J.A. Bennett, N.J. Creamer, L.E. Macaskie, L. Bodeness, in: 8th World Congress of Chem. Eng., Montreal, Canada, 2009.

[102] R.A. Rajadhyaksha, S.L. Karwa, Solvent effects in catalytic-hydrogenation, Chemical Engineering Science, 41 (1986) 1765-1770.

[103] M.A. Henderson, The interaction of water with solid surfaces: fundamental aspects revisited, Surface Science Reports, 46 (2002) 1-308.

[104] T.R. Mattsson, S.J. Paddison, Methanol at the water-platinum interface studied by ab initio molecular dynamics, Surface Science, 544 (2003) L697-L702.

[105] D. Cao, G.Q. Lu, A. Wieckowski, S.A. Wasileski, M. Neurock, Mechanisms of methanol decomposition on platinum: A combined experimental and ab initio approach, Journal of Physical Chemistry B, 109 (2005) 11622-11633.

[106] I. Horiuti, M. Polanyi, Exchange reactions of hydrogen on metallic catalysts, Transactions of the Faraday Society, 30 (1934) 1164-1172. 
[107] P. Hohenberg, W. Kohn, Inhomogeneous electron gas, Physical Review, 136 (1964) 864-871.

[108] N.K. Sinha, M. Neurock, Chem. Cat. Chem. submitted for publication.

[109] W.G. Hoover, Canonical Dynamics - Equilibrium Phase-Space Distributions, Physical Review A, 31 (1985) 1695-1697.

[110] S. Nose, A Unified Formulation of the Constant Temperature Molecular-Dynamics Methods, Journal of Chemical Physics, 81 (1984) 511-519.

[111] S.K. Desai, M. Neurock, K. Kourtakis, A periodic density functional theory study of the dehydrogenation of methanol over Pt(111), Journal of Physical Chemistry B, 106 (2002) 2559-2568.

[112] C.M. Grimaud, D. Radosavkic, S. Ustaze, R.E. Palmer, Vibrational spectroscopy of cinnamaldehyde on graphite and supported Pd islands, Applied Surface Science, 178 (2001) 1-6.

[113] A. Iyer, H. Galindo, S. Sithambaram, C. King'ondu, C.-H. Chen, S.L. Suib, Nanoscale manganese oxide octahedral molecular sieves (OMS-2) as efficient photocatalysts in 2-propanol oxidation, Applied Catalysis a-General, 375 (2010) 295-302.

[114] M.L. Toebesa, F.F. Prinsloob, J.H. Bittera, A.J.v. Dillena, d.J.K. P, Influence of oxygen-containing surface groups on the activity and selectivity of carbon nanofiber-supported ruthenium catalysts in the hydrogenation of cinnamaldehyde, Journal of Catalysis, 214 (2003) 78-87.

[115] T. Gao, M. Glerup, F. Krumeich, R. Nesper, H. Fjellvag, P. Norby, Microstructures and spectroscopic properties of cryptomelane-type manganese dioxide nanofibers, Journal of Physical Chemistry C, 112 (2008) 13134-13140.

[116] Y. Li, H. Xie, J. Wang, L. Chen, Preparation and electrochemical performances of alpha- $\mathrm{MnO}_{2}$ nanorod for supercapacitor, Materials Letters, 65 (2011) 403-405.

[117] H. Ma, J. Shen, M. Shi, B. Yan, N. Li, M. Ye, Facile and template-free preparation of alpha-MnO(2) nanostructures and their enhanced adsorbability, Materials Research Bulletin, 46 (2011) 1461-1466.

[118] E. Cockayne, L. Li, First-principles DFT+U studies of the atomic, electronic, and magnetic structure of alpha-MnO2 (cryptomelane), Chemical Physics Letters, 544 (2012) 53-58.

[119] J.P. Perdew, A. Ruzsinszky, G.I. Csonka, O.A. Vydrov, G.E. Scuseria, L.A. Constantin, X. Zhou, K. Burke, Restoring the density-gradient expansion for exchange in solids and surfaces, Physical Review Letters, 100 (2008).

[120] P.E. Blochl, Projector Augmented-Wave Method, Physical Review B, 50 (1994) 17953-17979.

[121] G. Kresse, D. Joubert, From ultrasoft pseudopotentials to the projector augmented-wave method, Physical Review B, 59 (1999) 1758-1775.

[122] S.L. Dudarev, G.A. Botton, S.Y. Savrasov, C.J. Humphreys, A.P. Sutton, Electron-energy-loss spectra and the structural stability of nickel oxide: An LSDA+U study, Physical Review B, 57 (1998) 15051509.

[123] K.D. Kwon, K. Refson, G. Sposito, Defect-induced photoconductivity in layered manganese oxides: A density functional theory study, Physical Review Letters, 100 (2008). 
[124] I.X. Green, W. Tang, M. Neurock, J.T. Yates, Insights into Catalytic Oxidation at the $\mathrm{Au} / \mathrm{TiO}_{2}$ Dual Perimeter Sites, Accounts of Chemical Research, (2014) DOI: 10.1021/ar400196f.

[125] M. Boudart, M.A. Vannice, J.E. Benson, Adlineation Portholes Spillover, Zeitschrift Fur Physikalische Chemie-Frankfurt, 64 (1969) 171-177.

[126] J.M. Herrmann, Electronic processes in hydrogen spillover, Studies in Surface Science and Catalysis, 138 (2001) 189-196.

[127] R. Prins, V.K. Palfi, M. Reiher, Hydrogen Spillover to Nonreducible Supports, Journal of Physical Chemistry C, 116 (2012) 14274-14283.

[128] W.C. Conner, J.L. Falconer, Spillover in Heterogeneous Catalysis, Chemical Reviews, 95 (1995) 759788.

[129] H. Liu, M. Yuan, C. Guo, R. Li, H. Fu, C. Huab, X. Li, Selective Hydrogenation of Cinnamaldehyde to Cinnamyl Alcohol over Ru/ZrO2.xH2O Catalyst, Chinese Journal of Catalysis, 32 (2011) 1256-1261.

[130] C.-Y. Hsu, T.-C. Chiu, M.-H. Shih, W.-J. Tsai, W.-Y. Chen, C.-H. Lin, Effect of Electron Density of Pt Catalysts Supported on Alkali Titanate Nanotubes in Cinnamaldehyde Hydrogenation, The Journal of Physical Chemistry C, 114 (2010) 4502-4510.

[131] W. Koo-amornpattana, J.M. Winterbottom, Pt and Pt-alloy catalysts and their properties for the liquidphase hydrogenation of cinnamaldehyde, Catalysis Today, 66 (2001) 277-287. 